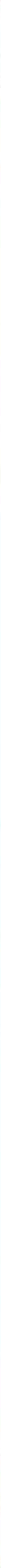




\title{
Elasticity and Wave Propagation in Granular Materials
}

\author{
Kianoosh Taghizadeh Bajgirani
}




\section{Samenstelling promotiecommissie:}

Prof. dr. ir. G.P.M.R. Dewulf

Universiteit Twente, voorzitter/secretaris

Prof. dr. rer.-nat. S. Luding

Universiteit Twente, promotor

Dr. V. Magnanimo

Universiteit Twente, promotor

Prof. dr. ir. T. van den Boogaard

Universiteit Twente

Prof. dr. ir. A. de Boer

Universiteit Twente

Prof. dr.-ing. H. Steeb

Universität Stuttgart

Prof. dr. J. Jenkins

Cornell University

Prof. dr. G. Combe

Laboratoire 3SR

The work in this thesis was carried out at the Multi Scale Mechanics (MSM) group of the Faculty of Science and Technology of the University of Twente. It is part of the research program T-MAPPP (http://www.t-mappp.eu/), which is financially supported by the European Union funded Marie Curie Initial Training Network, FP7 (ITN-607453).

\section{T.MAOQP UNIVERSITY OF TWENTE. IIST}

Cover design by Hamidreza Madadi.

Printed by Ipskamp Printing, Enschede, The Netherlands.

Copyright (C) 2019 by Kianoosh Taghizadeh Bajgirani.

All rights reserved. No part of the material protected by this copyright notice may be reproduced or utilized in any form or by any means, electronic or mechanical, including photocopying, recording or by any information storage and retrieval system, without written permission of the author.

ISBN: 978-90-365-4860-1

DOI number: 10.3990/1.9789036548601

Official URL: https ://doi .org/10.3990/1.9789036548601 


\title{
ELASTICITY AND WAVE PROPAGATION IN GRANULAR MATERIALS
}

\author{
PROEFSCHRIFT
}

ter verkrijging van

de graad van doctor aan de Universiteit Twente, op gezag van de rector magnificus, prof. dr. T.T.M. Palstra

volgens besluit van het College voor Promoties in het openbaar te verdedigen donderdag 26 September 2019 om 12.45 uur

door

Kianoosh Taghizadeh Bajgirani

geboren op 27 augustus 1990

Tehran, Iran 
Dit proefschrift is goedgekeurd door de promotor:

Prof. dr. rer.-nat. S. Luding

en de assistent-promotor:

Dr. V. Magnanimo 
Dedicated to my family and friends 



\section{Contents}

Abstract $\quad$ xi

Samenvatting xiii

1 Introduction 1

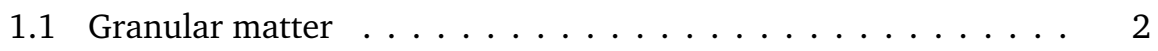

1.1 .1 Granular mixtures . . . . . . . . . . . . . . 3

1.1.2 Discrete Element Method . . . . . . . . . . . . 4

1.1.3 Micro-macro transition . . . . . . . . . . . . 5

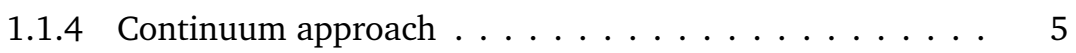

1.2 Elasticity in granular materials . . . . . . . . . . . . 7

1.2.1 Elasticity and State variables ................ 9

1.3 Waves propagation in granular medium . . . . . . . . . . 10

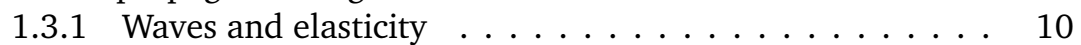

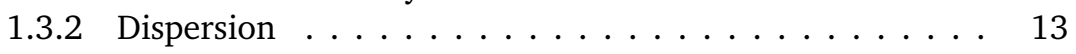

1.3 .3 Attenuation (loss of energy) . . . . . . . . . . . . . 13

1.3 .4 Master equation . . . . . . . . . . . . . . . . . . . 14

1.4 Thesis scope and outline . . . . . . . . . . . . 15

2 Micro- and macro-mechanical study of spherical granular particles 19

2.1 Introduction . . . . . . . . . . . . . . . 20

2.2 Simulation approach . . . . . . . . . . . . . . . . 22

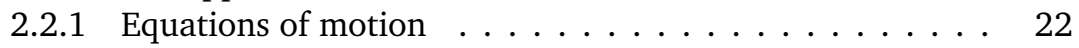

2.2 .2 Contact model . . . . . . . . . . . . . 23 
2.2 .3 Frictional contact model . . . . . . . . . . . . . . 24

2.2.4 Adhesive, elasto-plastic contact model . . . . . . . . . . . . 24

2.2 .5 Microscopical quantities . . . . . . . . . . . . . . 25

2.3 Preparing samples and defining quantities . . . . . . . . . . . . 29

2.3.1 Jamming transition (from fluid- to solid-like behavior) . . 30

2.3.2 Macro-quantities during the sample preparation . . . . . 31

2.4 Small strain stiffness . . . . . . . . . . . . . . . . 37

2.4.1 Incremental response of frictional samples . . . . . . . . . 38

2.4.2 Reversibility - from elastic to plastic . . . . . . . . . . 41

2.4.3 Incremental response of cohesive samples . . . . . . . . 43

2.5 Concluding remarks . . . . . . . . . . . . . . 45

3 Micromechanical study of the elastic stiffness in isotropic frictional granular solids

3.1 Introduction . . . . . . . . . . . . . . . . . . 48

3.2 Numerical setup . . . . . . . . . . . . . . . . 50

3.2 .1 Contact models . . . . . . . . . . . . . . 50

3.2 .2 Simulation parameters . . . . . . . . . . . . 51

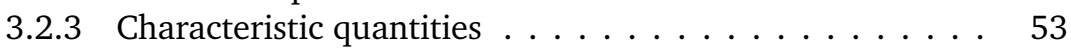

3.3 DEM simulations . . . . . . . . . . . . . . . . . . 54

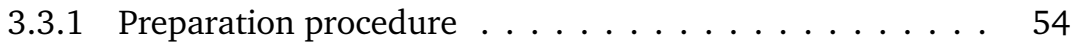

3.3.2 Elastic stiffness . . . . . . . . . . . . . . 55

3.3.3 Influence of inter-particle contact friction during preparation on the elastic moduli . . . . . . . . . . . . 57

3.4 Granular elasticity . . . . . . . . . . . . . . . . . . . 58

3.4.1 Effective medium theory . . . . . . . . . . . . 60

3.4 .2 Fluctuation theory . . . . . . . . . . . . . . 60

3.5 Role of the tangential stiffness during probing . . . . . . . . 63

3.5.1 Incremental non-affine fluctuations . . . . . . . . . . . 67

3.6 Concluding remarks . . . . . . . . . . . . . . . 69

4 Elastic waves in particulate glass-rubber mixtures $\quad 75$

4.1 Introduction . . . . . . . . . . . . . . . 76

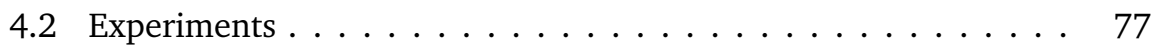

4.2 .1 Test procedure . . . . . . . . . . . . . 77

4.2 .2 Mixture properties . . . . . . . . . . . . 79

4.3 P-wave velocity . . . . . . . . . . . . . . . . . . . 81

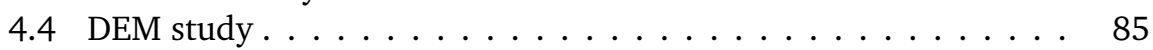

4.4 .1 Numerical setup . . . . . . . . . . . . 85 
4.4 .2 Numerical results $\ldots \ldots \ldots \ldots \ldots$. . . . . . . . . 87

4.5 Frequency spectrum $\ldots \ldots \ldots \ldots \ldots$

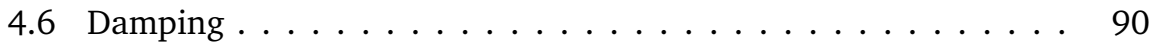

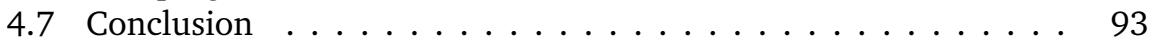

5 Stress based multi-contact model for discrete-element simulations 97

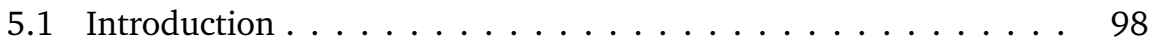

5.2 Discrete Element Method . . . . . . . . . . . . . . . 100

5.2 .1 Normal contact law . . . . . . . . . . . . . . . . . . 101

5.2 .2 Tangential force law . . . . . . . . . . . . . . . . 102

5.3 Deformable particle models . . . . . . . . . . . . . . 103

5.3.1 Multi-contact strain based model . . . . . . . . . . . . 103

5.3.2 Multi-contact stress based model . . . . . . . . . . . . . 104

5.3 .3 Modeling test cases . . . . . . . . . . . . . . 106

5.4 Uniaxial unconfined compression of a single rubber sphere . . 110

5.5 Uniaxial confined compression . . . . . . . . . . . . . . 111

5.5.1 Compression using hydrogel balls . . . . . . . . . . . 111

5.5.2 Compression using rubber spheres . . . . . . . . . . . 114

5.5.3 Compression using glass beads . . . . . . . . . . . . . 114

5.6 Computational cost performance of Mutli-contact models . . . . . 117

5.7 Conclusions and outlooks . . . . . . . . . . . . . . . . 117

6 Effect of Mass Disorder on Bulk Sound Wave Speed: A Multiscale Spectral Analysis 121

6.1 Introduction . . . . . . . . . . . . . . . . . . . 122

6.2 Granular Chain Model . . . . . . . . . . . . . . . . . . 123

6.2 .1 Linearized Equation of Motion . . . . . . . . . . . . 125

6.2 .2 Impulse Propagation Condition . . . . . . . . . . . . . . 127

6.2 .3 Standing Wave Condition . . . . . . . . . . . . . . . 127

6.2.4 Mass Disorder/Disorder Parameter $(\xi)$ \& Ensembles . . . 128

6.3 Energy Evolution . . . . . . . . . . . . . . . . . . . . . . . . 129

6.3.1 Total Energy in the Wavenumber Domain . . . . . . . . 130

6.3.2 Numerical Master Equation . . . . . . . . . . . . . . . 131

6.4 Results and discussion . . . . . . . . . . . . . . . . . 132

6.4.1 Energy Propagation with Distance . . . . . . . . . . . 132

6.4.2 Energy propagation in space and time . . . . . . . . . 137

6.4.3 Energy propagation across wave numbers . . . . . . . 139

6.4 .4 Attenuation . . . . . . . . . . . . . . . . . . . . . . 142

6.4.5 Reverse Modeling Frequency Propagation . . . . . . . . . 144 
6.5 Conclusion . . . . . . . . . . . . . . . . . . 147

7 Overview on continuum modeling of granular materials 155

7.1 Introduction . . . . . . . . . . . . . . 156

7.2 Overview on continuum modeling . . . . . . . . . . . . 157

7.2.1 Continuum model: a particle-based hypoelastic law . . . 165

7.3 Conclusion . . . . . . . . . . . . . . . . . . 169

8 Conclusion and Outlook 171

$\begin{array}{lr}\text { Summary } & 205\end{array}$

$\begin{array}{lr}\text { Acknowledgments } & 209\end{array}$

$\begin{array}{lr}\text { Curriculum Vitae } & 213\end{array}$ 



\section{Abstract}

Particle simulations are able to model behavior of granular materials, but are very slow when large-scale phenomena and industrial applications of granular materials are considered. Even with the most advanced computational techniques, it is not possible to simulate realistic numbers of particles in large systems with complex geometries. Thus, continuum models are more desirable, where macroscopic field variables can be obtained from a micro-macro averaging procedure. However, aspects of microscopic scale are neglected in classical continuum theories (restructuring, geometric non linearity due to discreteness, explicit control over particle properties).

The focus of this work is the investigation of elastic and dissipative behavior of isotropic, dense assemblies. In particular, the attention is devoted on the effect of microscopic parameters (e.g. stiffness, friction, cohesion) on the macroscopic response (e.g. elastic moduli, attenuation). The research methodology combines experiments, numerical simulations, theory.

One goal is to extract the macroscopic material properties from the microscopic interactions among the individual constituent particles; for simple enough systems this can often be done using techniques from mechanics and statistical physics. While these simplified models can not capture all aspects of technically relevant realistic grains the fundamental physical phase transitions can be studied with these model systems.

Complex mixtures with more than one particle species can exhibit enhanced mechanical properties, better than each of the ingredients. The interplay of soft with stiff particles is one reason for this, but requires a more accurate formation of the interaction of deformable spheres. A new multi-contact approach is proposed which shows a better agreement between experiments and simulations in comparison to the conventional pair interactions.

The study of wave propagation in granular materials allows inferring many fundamental properties of particulate systems such as effective elastic and dis- 
sipative mechanisms as well as their dispersive interplay. Measurements of both phase velocities and attenuation provide complementary information about intrinsic material properties. Soft-stiff mixtures, with the same particle size, tested in the geomechanical laboratory, using a triaxial cell equipped with wave transducers, display a discontinuous dependence of wave speed with composition.

The diffusive characteristic of energy propagation (scattering) and its frequency dependence (attenuation) are past into a reduced order model, a master equation devised and utilized for analytically predicting the transfer of energy across a few different wavenumber ranges, in a one-dimensional chain. 


\section{Samenvatting}

Door de deeltjes van granulaire materiaal te simuleren zijn we in staat het bulkgedrag te modelleren. Echter zijn dit soort deeltje simulaties zeer langzaam wanneer er grootschalige fenomenen en industriële toepassingen worden gesimuleerd. Zelfs met de meest geavanceerde computertechnieken is het niet mogelijk om met een realistische hoeveelheid deeltjes deze simulaties toe te passen in complexe geometrieën. Dus continuüm modellen zijn nog steeds wenselijk, waarbij de macroscopische variabelen kunnen worden verkregen door micro-macro middeling methoden. Echter wordt de microscopische schaal vaak weggelaten in de klassieke continuüm theorieën. (Herstructurering, geometrische niet-lineariteit door discretisatie, expliciete controle over deeltjes eigenschappen)

De focus van dit werk is het onderzoek naar de elastische en dissipatieve gedrag van isotropische, dichtgepakte samenstellingen. Specifiek wordt er aandacht besteed aan het effect van microscopische parameters (stijfheid, wrijvingsweerstand, cohesie) op het macroscopische gedrag (elasticiteitsmodulus, verzwakking). De onderzoeksmethodiek combineert hierbij experimenten, numerieke simulaties en theorie.

Een van de doelen is het verkrijgen van macroscopische materiaaleigenschappen uit de microscopische interacties tussen de individuele deeltjes in samenstellingen. Voor eenvoudige systemen kan dit worden gedaan door bestaande technieken uit stijfheid en sterkteleer en statistische natuurkunde. Hoewel deze eenvoudige modellen niet alle aspecten van de technische relevante realistische granulaire materialen kan bevatten, kan met deze modellen de fundamentele fase-transformaties worden onderzocht.

Complexe mengsels met meer dan een soort van deeltjes vertonen soms verbeterde mechanische eigenschappen dan dat de materialen apart zouden doen. Het samenspel tussen de zachte en harde deeltjes is een van de reden voor 
dit gedrag, echter vergt dit een betere omschrijving van de interactie tussen de deeltjes. Een nieuwe Multi-contact methode wordt uiteengezet waarbij een betere overeenkomst tussen experimenten en simulaties is gevonden dan dat er met conventionele interacties tussen twee deeltjes.

Door de studie naar de prorogatie van geluid in granulaire materialen kunnen we vele fundamentele eigenschappen van een granulair systeem afleiden, zoals de effectieve elastische en dissipatieve mechanismen en de verstrooiing. Metingen van beide fase snelheden en verzwakking geven daarnaast extra informatie over de belangrijke materiaaleigenschappen. Zacht-hard mengsels, met dezelfde deeltjesgrootte, zijn getest in een geo-mechanisch laboratorium doormiddel van een triaxial-cell met extra golf omvormers laten een onderbreking zien in de afhankelijkheid van de geluidssnelheid met de granulaire mengsels.

Het diffuse karakter van de energie stroming (verstrooiing) en de frequentieafhankelijkheid (verzwakking) zijn verzameld in een lagere order model, een alomvattende vergelijking ontwikkeld voor het analytisch voorspellen van de energie overbrenging over een bereik van meerdere golfgetallen in een één dimensionale ketting. 



\section{Chapter 1}

\section{Introduction}

The children of Adam are limbs of a whole

Having been created of one essence.

When the calamity of time afflicts one limb

The other limbs cannot remain at rest.

If you have no sympathy for the troubles of others;

You are not worthy to be called by the name of "human".

Saadi

Many industrial and geotechnical applications that are crucial for our society involve granular systems at small strain levels. That is the case of structures designed to be far from failure (e.g. shallow foundations or underlying infrastructure), strains in the soil are small and a sound knowledge of the bulk stiffness is essential for the realistic prediction of ground movements. In the following, a general introduction to granular matter is given. Then, different regimes under deformation of a bulk granular materials and the emergence of a theoretical framework based on micro-mechanical information to represent elastic behavior for granular materials are explained. Definition of elastic waves in solids is provided and some characteristics of mechanical waves in disordered heterogeneous media like attenuation, dispersion, and stochastic modeling are introduced. Finally, an outline of the thesis will be given. 


\subsection{Granular matter}

Granular materials (e.g. Fig. 1.1), though ubiquitous in nature and widely used in engineering and construction, remain relatively poorly understood. They may behave like solids, liquids or gases, though typically exhibiting a variety of unexpected behaviors that are not encountered in these conventional forms of materials. The preponderance of problems yet to be solved has sparked a renewed interest in granular materials, in different communities.

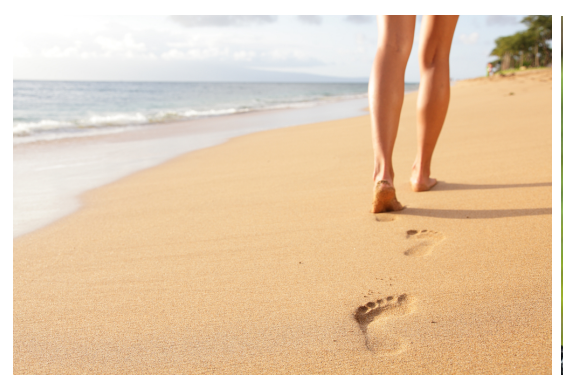

(a)

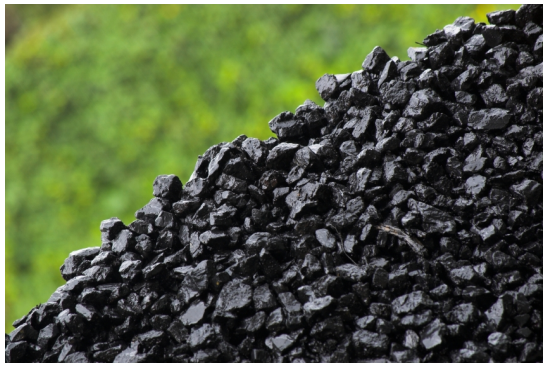

(b)

Figure 1.1: Examples of granular materials in our daily life.

Granular materials consist of discrete particles such as, e.g., separate sandgrains, agglomerates (made of many primary particles), natural solid materials like sandstone, or ceramics, metals or polymers sintered during additive manufacturing. The primary particles can be as small as nano-meters, micro-meters, or millimeters, [153] covering multiple scales in size and a variety of mechanical and other interaction mechanisms like, e.g., friction and cohesion [263]. The latter becomes more and more important the smaller the particles are. All those particle systems have a particulate, usually disordered, possibly inhomogeneous and often anisotropic micro-structure, which is at the core of many of the challenges one faces when trying to understand powder technology and granular matter.

Particle systems as bulk show a completely different behavior as one would expect from the individual particles. Collectively, particles either flow like a fluid or rest static like a solid. In the former case, for rapid flows, granular materials are collisional and inertia dominated and compressible similar to a gas. In the latter case particle aggregates are solid-like and thus can form, e.g., sand piles or slopes that do not move for long time. In between is the dense 
and slow flow regime that connects the extremes and is characterized by the transitions $(i)$ : from static to flowing (failure, yield) or vice-versa $(i i)$ : from fluid to solid (jamming). On the particle and contact scale, the most special property of particle systems is their dissipative, frictional, and possibly cohesive nature. Here, dissipation means that kinetic energy at this scale is irrecoverably lost from the particles translational degrees of freedom into heat in terms of random motion, or due to plastic, i.e. irreversible, deformations either of the particles, or of the micro-structure (chapter 2). The transition from fluid to solid can be caused by dissipation alone, which tends to slow down motion. The transition from solid to fluid (start of flow) is due to failure and instability, when dissipation is not strong enough to avoid the solid yielding and transits to a flowing regime.

\subsubsection{Granular mixtures}

Granular matter usually occur in various sizes, or as mixtures of different materials (Fig. 1.2). Particulate mixtures are of interest for a large number of fields, materials, and applications, including mineral processing, environmental engineering, geomechanics and geophysics, and have received a lot of attention in the last decades [148]. A specific example in geotechnical engineering is the increasing incorporation of recycled materials (e.g. shredded or granulated rubber, crushed glass) often used into conventional designs and soil improvement projects [20, 64, 134]. Moreover, sophisticated mixtures of asphalt and concrete are widely used to construct roads and, also here, mixing-in additional components is a widely applied option [85, 86, 222, 264, 268, 274].

Among those mixtures, binary mixtures of two materials are a particularly interesting selection. Binary granular mixtures comprise a wide range of natural and industrial materials, whose mechanical and acoustic behavior is strongly influenced by the relative amount of the components $[53,274]$. While several researchers have investigated bidisperse mixtures [123, 273], the investigation of mixtures made of two different components with different material properties (densities, visco-elastic moduli) is limited so far to phenomenological observations; a deeper insight into the governing micromechanical properties is still missing [4, 33, 39]. Through better understanding the underlying small-scale physics, the effective behavior of mixtures can be robustly predicted, tailored to specific technical applications and even optimized on demand.

Much more limited is the work on mixtures made of a stiff and soft phase. This was addressed experimentally in the substantial work of diffeent authers $[108,135,251]$ and numerically in $[61,217,269]$ and with special focus on the 


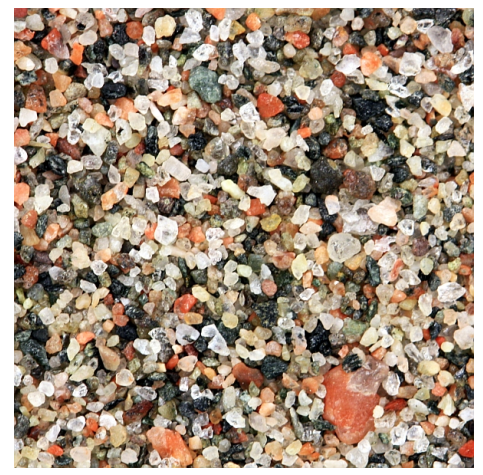

Figure 1.2: Examples of granular mixtures in our daily life.

(post-)liquefaction behaviour. In a recent contribution we have combined wave propagation experiments (chapter 4) and DEM simulations (chapter 4 and 5) to show how the bulk modulus in a granular soil increases if soft inclusions are added in proper amount. This is a field of immense interest, as interrelation between solid phases at the microscale opens up many possibilities [154].

\subsubsection{Discrete Element Method}

In recent decades, the discrete element method (DEM) [41] that models the motion and interaction of many individual particles has become increasingly popular as a computational tool to model granular systems in both academia and industry. To date, not only due to increasing computer power available, considerable scientific advances have been made in the development of particle simulation methods, resulting in an increasing use of DEM. However, careful verification of the various numerical codes and validation of the simulation results with closely matching experimental data is essential to establish DEM as a widely accepted tool able to produce satisfactory quantitative predictions with added value for design, optimization and operation of industrial processes.

With the development of computational power in recent years, the discrete particle/element method has gained its focus to the simulation community. However, this method has its own limitations in applying to the real world. One part is that the number of particles can be simulated is limited, normally in the order between $10^{4}$ to $10^{7}$ in a 3D setup, whereas one normally has much more than $10^{9}$ to $10^{11}$ particles in a real system. Another point is the complexity of de- 
scribing the real contact mechanics between particles in a numerical model. One has to make several assumptions to reduce this complexity to be able to simulate many particles and all their contacts. Nevertheless, the DEM/DPM method is a really helpful tool for understanding the granules bulk behavior qualitatively (and quantitatively) and thus one can explore the physics behind the scene, for discrete particulate systems, which the traditional continuum solid/fluid mechanics can not explain.

\subsubsection{Micro-macro transition}

Due to their wide application, granular media have received a lot of attention in many fields, such as soil mechanics, process engineering, mechanical engineering, material science and physics. Attempts to model these systems with classical continuum theory and standard numerical methods and design tools cannot always be successful, because of their discreteness and disorder at the microscopic scale. Therefore, it is necessary to employ a multi-scale approach that can link the discrete nature of granular systems to a macroscopic, continuum description. Both fundamental understanding and design/operation of unit processes and plants require multi-scale and multiphase approaches, where the discrete nature of the particles is of utmost relevance and must not be ignored. Fig. 1.3 llustrates the idea behind the micro-macro transition, i.e., passing information from discrete element (particulate) to finite element (continuum) simulations.

\subsubsection{Continuum approach}

DEM simulations are very detailed and therefore slow when large-scale phenomena and industrial applications of granular materials are considered. Even with the most advanced computational techniques of today, it is not possible to simulate realistic numbers of particles with complex geometries. Thus, continuum models are more desirable, where a granular medium is assumed a continuum, and principles of continuum mechanics are applied to obtain macroscopic field variables. However, besides the speed advantage of a continuum approach, many features of granular materials at the microscopic scale have to be neglected, such as restructuring, geometric non-linearity due to discreteness, or explicit control over particle properties. The mechanical behavior of the materials has to be defined, for example, based on the relation between stress and strain extracted from continuum models [74]. 


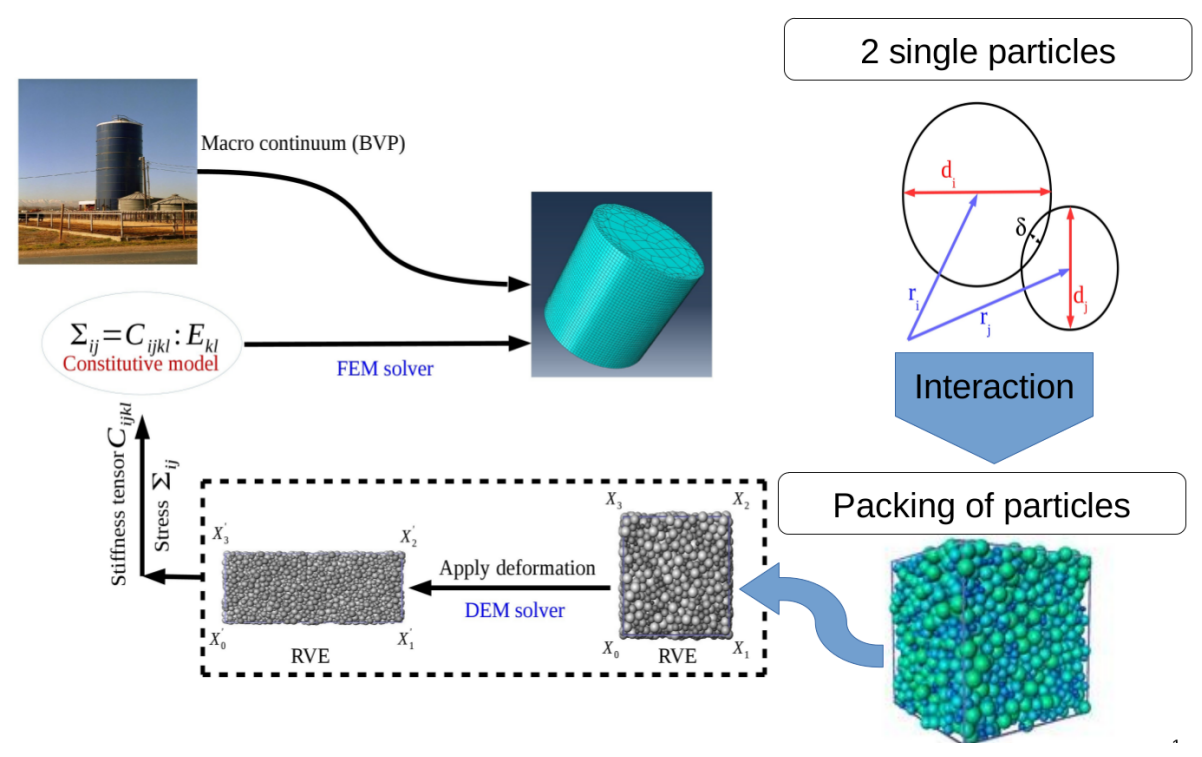

Figure 1.3: Micro-macro concept to link between discrete to continuum.

The relation between stresses and strains is called constitutive model and it depends on the mechanical properties of the materials. Constitutive models are formulated mathematically and modeled phenomenologically in continuum mechanics. Different varieties of constitutive models have been established to describe the material behavior and the deformation, such as elasticity, plasticity, visco-elasticity, creep and etc. Several constitutive models within the framework of continuum mechanics have been developed to describe the mechanical behavior of granular materials. Most standard models with wide range of application, such as elasticity, elasto-plasticity, or fluid-/gas-models of various kinds are commonly used for granular flows. Nevertheless, they are sometimes valid, only in a very limited range of parameters and flow conditions. For example, the framework of kinetic theory is an established tool with quantitative predictive value for rapid granular flows but it is not applicable in dense, quasi-static and static cases [145]. Further models, such as hyper-or hypo-elasticity, are complemented by hypo-plasticity and the so-called granular solid hydrodynamics, where it has been established to represent also the mechanical behavior of granular solids. Differently from classical plasticity theory, where a plastic yield 
surface can be defined, the granular solid models provide incremental evolution of equations with strain, and involve limit states, because a strict split between elastic and plastic behavior seems invalid in granular materials. Some of these theories have been extended to accommodate the anisotropy of the microstructure [146], but only few models account for an independent evolution of the microstructure. The anisotropy constitutive model based on incremental evolution equations for stress and fabric was presented in Ref. [121] for frictionless systems.

\subsection{Elasticity in granular materials}

It is commonly known that soil behavior is not as simple as its prediction with simply formulated linear constitutive models, which are commonly and carelessly used in numerical analyses. Complex soil behavior which stems from the nature of the multi-phase material exhibits both elastic and plastic nonlinearities. Deformations include irreversible plastic strains. Depending on the history of loading, soil may compact or dilate, its stiffness may depend on the magnitude of stress levels, and soil deformations can be time-dependent, etc.

The behavior of granular materials depends on the strain regime. Roughly speaking, we can distinguish $(i)$ : an elastic regime (very small strain); $(i i)$ : an elasto-plastic regime at intermediate and large strain; and $(i i i)$ : a fully plastic regime where the material flows at constant stress and volume (beyond the solid to fluid transition).

A stiffness degradation curve, obtained in the resonant column device, is normally used to explain the shear stiffness $G$ for a wide range of shear strain. A typical output of the resonant column experiment is given in Fig.1.4.

Atkinson and Sallfors used a normalized stiffness degradation curve to categorize the strain levels into three groups (as shown in Fig.1.4): the very small strain level, where the stiffness modulus is constant in the elastic range; the small strain level, where the stiffness modulus varies non-linearly with the strain; and the large strain level, where the soil is close to failure and the soil stiffness is relatively small $[14,157]$.

For many geotechnical structures under working loads, the deformations are small. The regime of deformation where the behavior can be considered linear elastic is infinitesimal, with nonlinear and irreversible effects present already at small strains. Nevertheless, the stiffness of soils is of outmost importance, as it provides an anchor on which to attach the subsequent stress-strain response $[24,175]$. 
An elastic response is only observed for very small strain (order of $10^{-6}$ or $10^{-5}$ ) intervals, and should in fact be viewed as an approximation, as dissipation mechanisms are always present (in particular, solid friction) and preclude the general definition of an elastic energy (chapter 2). The relative amount of dissipation decreases as the size of the probed strain interval approaches zero. For that reason, the material behavior is best characterized as "quasielastic" in that limited range $[24,35]$. In fact, soil behavior is considered to be truly elastic in the range of very small strains, where soil even may exhibit a nonlinear stress-strain relationship. However, its stiffness is almost fully recoverable after unloading. Following the pre-failure non-linearities of soil, one may observe a strong variation of stiffness starting from very small shear strains, which cannot be reproduced by models such as the linear-elastic Mohr-Coulomb model.

Fig.1.4 shows that the response of granular materials is nonlinear and inelastic even at extremely small strains. The region of stress or strain in which granular materials can be described as truly elastic, producing an entirely recoverable response to perturbations, the so-called small-strain stiffness $G_{\max }$, is very small, corresponding to shear strains of the order of $10^{-4}$. In turn, the size of the elastic, reversible regime depends on material characteristics, stress state, anisotropy: the elastic range increases with increasing asperities (particle friction) and pressure, but decreases with increasing anisotropy.

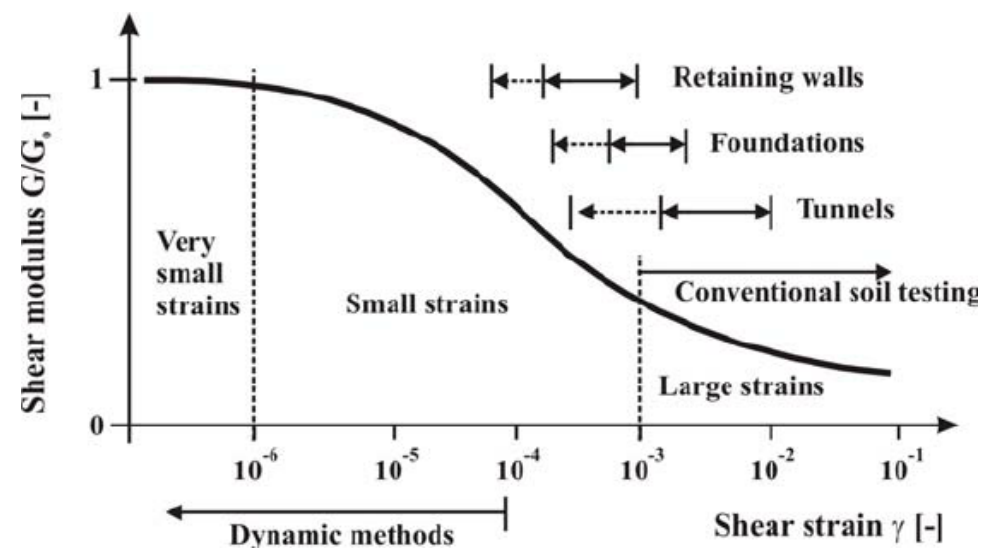

Figure 1.4: Degradation curve for $G$ with indication of typical soil tests and geotechnical applications per strain regime [14, 157]. 


\subsubsection{Elasticity and State variables}

Despite the long-standing debate across the geomechanical, mechanical and physics communities, basic features of the physics of granular elasticity are currently unresolved, like a proper set of state variables to characterize the effective moduli.

In early studies, macroscopic variables measurable in laboratory experiments, were thought to be sufficient. Based on those information, many empirical relations have been proposed, where the elastic moduli are functions of pressure and void ratio, e.g. [19, 80, 81, 201]. However, such formulations miss a first order mechanical interpretation and coefficients have to be backcalculated case by case from experiments, based on the specific material and stress path. Moreover, experimental evidences [30, 54, 124, 191], along with many numerical studies $[3,160]$, show that stress and volume fraction are not sufficient to characterise granular elasticity

On the other hand, conventional approaches in the framework of solid state elasticity [139] consider a uniform strain at all scales, with the displacement field of the grains following the macroscopic deformation (affine approximation). These Effective Medium Theories (EMT) developed by Digby and Walton $[47,253]$ in the 80 's are the first, simplest attempt for a micromechanical approach to the elasticity of granular soils. EMT predicts the moduli of an isotropic granular material in terms of the external pressure, the void ratio and average coordination number $\left(p_{0}, e, Z\right)$. In particular, the pressure dependency is $G \sim K \sim p_{0}^{1 / 3}$, a direct consequence of the Hertz interaction between the particles. However such scaling is not recovered in experiments and previous analysis (see [70] for a review) raises serious question about the validity of these generally accepted theoretical elastic formulations.

Empirical relations coming from experiments and micromechanical EMT equations show many similarities and the two approaches can fruitful inform each other. Following one of the paths suggested already in [70] and further developed in $[155,158]$, the set of state variables to describe granular elasticity is complete, and experiments follow the trend predicted by the model. This is obtained with the aid of Discrete Element Simulations (DEM), that uniquely allow to monitor the kinematics at the microscale and link it with the macroscale.

However, the EMT framework still largely overpredicts the elastic moduli of loose samples, especially when shear is involved. The difficulty in describing theoretically the shear modulus is due to the complex relaxation of the particles as related to the structural disorder in the packing [158]. Sophisticated theories in which collective fluctuations and relaxation of the particles are explicitly 
accounted for are needed to recover quantitative agreement. Here we briefly illustrate the mechanics beyond these theories and compare the results with numerical simulations. Recent attempts in this direction are developed in [93, 125] where statistical parameters from a fluctuation analysis are introduced to describe the scaling of the moduli. In these theoretical models, fluctuations are introduced in the kinematics of contacting particles. They are determined as functions of "fabric tensors" that describe, on average, the packing geometry and the variation of the number of contacts per particle. The proposed theories are able to predict an elastic resistance of the aggregate comparable to numerical simulation (chapter 3 ).

\subsection{Waves propagation in granular medium}

A wave is an elastic perturbation that propagates between two points through a body (volume waves) or on the surface (surface waves) without material displacement [5]. Traveling through the interior of the Earth, body waves arrive before the surface waves and are at higher frequency than surface waves. They are divided into two types P- waves and S-waves, the P-waves traveling faster than $\mathrm{S}$-waves through a solid body.

In the case of volume waves the acoustic-elastic effect is related to the change in the wave velocity of small amplitude waves due to the stress state of the body. Differently from liquids, in a solid material three acoustic polarisations exist, more specifically a longitudinal and two transversal branches.

\subsubsection{Waves and elasticity}

Wave also offer a direct connection to elastic properties of materials, due to their relatively easy application, through commercial equipment, as well as the various formulations relating the wave velocity and the material moduli. Advanced features like frequency dependence of wave parameters may further improve the characterization capacity. Concrete and soil samples due to their inherent microstructure, (which is enriched by the existence of damage-induced cracking), exhibit interesting behaviors concerning the propagation of pulses of different frequencies. Here, we will derive the relations between the elastic characteristics and the velocities of acoustic waves, in the longitudinal and transversal directions.

The use of wave propagation to describe the small strain stiffness behavior of a material has been a well documented, widely-used technique, as evidenced 
in the literature. Velocity testing, which includes BE and UT technology, has been gaining popularity as an experimental method due to its relative ease of obtaining the modulus of a material.

Let us consider a three-dimensional body with density $\rho$, homogeneous, isotropic and elastic. The stress change due to the propagation of the wave in the body is given by the Newton's second law applied to the volume element $\rho d V$ [46]:

$$
\frac{\partial \sigma_{i j}}{\partial x_{j}}=\rho \ddot{u}_{i},
$$

with $\sigma_{i j}$ stress and $u_{i}$ displacement of the volume element in directions $i, j=$ $1,2,3$. On the other hand, the constitutive relation for the elastic body holds, that relates the stress tensor to the strain $\epsilon_{i j}$ via the stiffness tensor $C_{i j k l}$

$$
\sigma_{i j}=C_{i j k l} \epsilon_{k l}
$$

In the isotropic case, Eq.(1.2) becomes (Lamé equation)

$$
\sigma_{i j}=\lambda \Theta \delta_{i j}+2 G \epsilon_{i j}
$$

where $\Theta=\sum_{i=1}^{3} \epsilon_{i i}, G$ and $\lambda$ are the shear modulus and Lamé coefficient respectively, and the incremental strain tensor is given by

$$
\epsilon_{i j}=\frac{1}{2}\left(\frac{\partial u_{i}}{\partial x_{j}}+\frac{\partial u_{j}}{\partial x_{i}}\right)
$$

The bulk modulus is related to the previous quantities as $K=\lambda+2 / 3 G$.

Using Eqs.(1.3-1.4) in Eq.(1.1), the equation of motion becomes

$$
\rho \frac{\partial^{2} u_{i}}{\partial t^{2}}=\lambda \frac{\partial}{\partial x_{j}}\left(\frac{\partial u_{j}}{\partial x_{i}}\right)+G \frac{\partial^{2} u_{i}}{\partial x_{j}^{2}}+G \frac{\partial}{\partial x_{j}}\left(\frac{\partial u_{j}}{\partial x_{i}}\right) .
$$

From Helmholtz decomposition, the displacement vector $\boldsymbol{u}$ can be written in terms of a scalar potential $\phi$ and a vector potential $\psi$ :

$$
u=\nabla \phi+\nabla \times \psi,
$$

where the tensorial notation has been used for the sake of brevity. Thus Eq.(1.5) is

$$
\boldsymbol{\nabla}\left[\rho \frac{\partial^{2} \phi}{\partial t^{2}}-\left(\lambda+\frac{4}{3} G\right) \nabla^{2} \phi\right]+\boldsymbol{\nabla} \times\left[\rho \frac{\partial^{2} \boldsymbol{\psi}}{\partial t^{2}}-G \nabla^{2} \boldsymbol{\psi}\right]=\mathbf{0}
$$


Eq.(1.6) is known as the wave equation and predicts longitudinal and transversal modes of propagation. The first term in Eq.(1.6) depends only on $\phi$ and is related to the propagation of waves in the longitudinal direction, while the second term depends on the vector potential $\psi$ and is associated to the transversal waves. Both terms must be separately zero to satisfy Eq.(1.6), that is, the two propagation modes, longitudinal and transversal, are independent.

Finally, if we introduce the longitudinal and shear components of the displacement related to $\phi$ and $\psi$ respectively as

$$
\boldsymbol{u}_{P}=\boldsymbol{\nabla} \phi \quad \text { and } \quad \boldsymbol{u}_{S}=\boldsymbol{\nabla} \times \boldsymbol{\psi},
$$

from Eq.(1.6) we can derive the velocities of the longitudinal and transversal waves for the isotropic elastic body (chapter 4):

$$
V_{P}=\sqrt{\frac{(\lambda+4 / 3 G)}{\rho}} \quad \text { and } \quad V_{S}=\sqrt{\frac{G}{\rho}}
$$

Due to the properties of divergence and curl angular displacements and rotations are not allowed during propagation of longitudinal waves, and volume changes are forbidden for transversal waves.

When observing Eq.(1.7), some aspects appear: $(i)$ : the propagation velocity increases with the stiffness of the material and decreases with its mass density (inertia), these characteristics being constants in a given solid body; ( $i$ i) : the velocity of transversal waves is smaller than the velocity of longitudinal waves, given the relative values of the moduli.

We move now our attention from solid to particulate materials. When the wavelength is significantly longer than the internal scales of the material, such as particle or cluster size, the propagation velocity can be defined for the equivalent continuum, that is Eqs.(1.7), where the elastic moduli and mass density refer to the bulk medium. Differently, for high frequencies and short wavelengths, the continuum assumption does not hold, due to the heterogeneity of the material at small scale and forces fluctuation [228]. With increasing frequencies, features related to the multiscale nature of soils become dominant, e.g. dispersion and frequency filtering.

Other than frequency, also amplitude is an important factor to take into account. The propagation of elastic waves is, by definition, a small perturbation phenomenon that does not alter the micro-structure (fabric) or cause permanent (plastic) effects. This condition must be guaranteed for the continuum analogy to hold. If both conditions, long wavelength and small amplitude, are fulfilled, 
wave measurements (obtained e.g. via wave transducers) can be used to infer elastic moduli and vice versa.

\subsubsection{Dispersion}

The study of the dispersive behaviors of materials with respect to wave propagation is a central issue in modern mechanics. Dispersion is defined as that phenomenon for which the speed of propagation of waves in a given material changes when changing the wavelength (or, equivalently, the frequency) of the traveling wave. This is a phenomenon which is observed in practically all materials as far as the wavelength of the traveling wave is small enough to interact with the heterogeneities of the material at smaller scales. Dispersion most often refers to frequency-dependent effects in wave propagation. The dispersion relation describes the interrelations of wave properties like wavelength, frequency, velocities, refraction index and attenuation coefficient. In wave theory, dispersion is the phenomenon that the phase velocity of a wave depends on its frequency. Indeed, anyone knows that all materials are actually heterogeneous if considering sufficiently small scales: it suffices to go down to the scale of molecules or atoms to be aware of the discrete side of matter. It is hence not astonishing that the mechanical properties of materials are different when considering different scales and that such differences are reflected on the speed of propagation of waves.

\subsubsection{Attenuation (loss of energy)}

When a mechanical wave propagates through a medium, a gradual decay of wave amplitude can be observed before the wave diminishes, partly for geometric reasons because their energy is distributed on an expanding wave front, and partly because their energy is absorbed by the material they travel through. The energy absorption depends on the material properties. Amplitude is directly related to the acoustic energy or intensity of a sound. When sound travels through a medium, its intensity diminishes with distance. In certain materials, sound pressure (amplitude) is only reduced by the spreading of the wave. The effect produced is to weaken the sound. 'Scattering' is the reflection of the sound waves in directions other than its original direction of propagation. 'Absorption' is the conversion of the sound energy to other forms of energy. The combined effect of scattering and absorption is called attenuation of seismic waves and is an important characteristic in the modern seismology which needs to be studied. 
Seismic attenuation is commonly characterized by the quality parameter $Q$. It is most often defined in terms of the maximum energy stored during a cycle, divided by the energy lost during the cycle. Among the various methods of measuring attenuation from seismic data, the spectral ratio method is the most common method perhaps because it is easier to use and more stable.

\subsubsection{Master equation}

Including disorders, e.g. adding inclusions with different properties or size, will lead to enhanced absorption in typical frequency ranges/bands, as related to their specific characteristics relative to the basis material.

For instance, it is known that the dominant frequencies for exterior noise due to tire-road interactions lies in the range of 0.5 to $2 \mathrm{kHz}$. Research has shown that such noise generated in our daily life has a negative impact on humans health. Therefore, it is urgent to find a novel approach to damp as much as possible at exactly these unwanted frequencies. One way are high and wide walls/panels as usually installed along highways of metropolitan areas, which are generally expensive, while our approach involves a smarter design of the asphalt itself. Another example is the vibration and noise generated by railways or subways in the low frequency range (10 to $50 \mathrm{~Hz}$ ). These unwanted noises bring issues for specific infrastructures e.g. hospitals, art galleries, or tunnels, and could be avoided by a better composition and optimal use of the ballast or the concrete foundations with respect to their damping features. Last but not least, earthquakes are the natural hazard that generates the largest number of human casualties in our modern society. The dominant harmful frequency range of earthquakes usually remains very low, 5 to $20 \mathrm{~Hz}$ [99]. Earthquakes often cause unrecoverable damages to buildings and infrastructures and inestimable losses to our historical heritage. The cost of upgrade works on historical buildings is often too high and conflicts with other tight constraints. Our novel approach is to design a seismic protection ("cloaking") in the soil around, rather than on the building.

This will be the results from experiments and particle simulation to be in a macro-scale continuum model, with a resolution in frequency space. Instead of dealing with the too many eigen-modes of the system, we propose an approach with reduced complexity, where the frequencies are grouped in bands. This is different in spirit from reduced order modeling since one accounts for all frequencies, also the largest ones, but gives up the details by grouping all modes with similar frequency, gaining tremendous speed-up (chapter 6). 


\subsection{Thesis scope and outline}

To gain more insight into the micro-structure of granular materials, three dimensional discrete element simulations, theory, and experiments are performed on various quasi-static samples. Thus, this thesis is divided in chapters covering the elastic behavior of granular materials and considers many aspects such as mixtures of soft-stiff species, dissipation of energy, new approach on contact modelling of soft particles, master equation of a force chain.

- Chapter 2: In the next chapter of this dissertation, the micro- and macromechanical behavior of idealized granular assemblies are studied, comprising linearly elastic, frictional, cohesional, polydisperse spheres, in a periodic triaxial box geometry, using DEM. The stress response to various deformation modes, namely purely isotropic and deviatoric (volume conserving), applied to this granular samples are analyzed. A hysteretic contact model with plastic deformation and adhesion forces is used for micro- and macro-mechanical studies through fully disordered, densely packed, cohesive and frictional granular systems. Especially, the effect of friction and adhesion on the elastic response is examined.

- Chapter 3: Next, assemblies of polydisperse, linearly elastic frictional spheres are isotropically prepared using DEM . In a second stage, several static, relaxed configurations at various volume fractions above jamming are generated and tested. We investigate the effects of inter-particle contact properties on the elastic bulk and shear modulus by applying isotropic and deviatoric perturbations. The amplitude of the applied perturbations has to be small enough to avoid particle rearrangement and to get the elastic response, whereas large amplitudes develop plasticity in the sample due to contact and structure rearrangements between particles. We compare the data from DEM simulations with predictions from wellestablished micromechanical models, namely the Effective Medium Theory (EMT) and the Fluctuation Theory (FT). Both theories do not account for the effect of different preparation history (different inter-particle friction coefficients) on the elastic moduli. The fluctuation theory is in agreement with numerical data, almost perfect for the bulk modulus and close for the shear modulus, at least in the intermediate compression regime, but does not capture the anomalous behavior where the theory overpredicts.

- Chapter 4: This chapter carries out an extensive series of physical experi- 
ments in a triaxial cell set-up equipped with piezoelectric wave transducers. Conducting systematic experiments on various mixtures of particles with different species will help to complete the overall picture of the behavior of granular mixtures. The initial configuration considered is the most basic binary system of rubber (soft) and glass (stiff) particles randomly distributed within a latex membrane that allows to externally control the confining stress at different uni-axial compression levels. A wave is agitated on one side of such dense, static, mechanically stable packings and its propagation is investigated when it arrives at the opposite side. At various stress levels, P-wave has been excited and the time of flight is measured for many sample compositions and pressure levels.

- Chapter 5: The chapter attempts to model confined powder compaction with the discrete element method (DEM) is a really challenging task since classical particle-particle contact model are limited on the assumption of binary contacts regardless of the degree of confinement. In classical DEM, the fact that each particle experiences multiple simultaneous contacts that influence each other, at high relative densitites, is missing. Important progress has been made recently, resulting in the formulation of multicontact DEM but the picture is still incomplete. In this research, new force models tackling this issue are presented. By adding an extra term which is a function of Poisson's ratio and local particle stress tensor, we extend the classical force-displacement formula to capture pseudo deformation of particles. Hence, stress tensor commonly used for post-processing reasons (i.e. in cross coarse-graining methods) was used to account for multiple contacts acting simultaneously on a single particle. In our initial attempt, uniaxial compression simulations with Hertzian and linear contact models were conducted and modeled by frictionless spheres in the absence of gravitational forces. Comparisons between classical DEM simulations and the new, alternative model for interactions between multiple contacts are presented.

- Chapter 6: Focuses on the transfer energy with distance as well as across different wavenumbers, as the mechanical wave propagates. The diffusive characteristic of energy propagation has been discussed. A master equations is devised and utilized for analyzing the transfer energy across different wavenumbers, studied with the aid of a one-dimensional granular chain.

- Chapter 7: In this chapter a macroscopic continuum description is intro- 
duced for the granular material, based on microscale information. A constitutive model, for frictional particles, involving the elastic moduli and the relation between effective moduli and microstructure, is implemented in a Finite Element framework developed within the Kratos Multiphysics open source platform and some benchmark examples are carried out.

- Chapter 8: Finally, the last chapter dedicates to conclusions and recommendations for future research. 



\section{Chapter 2}

\section{Micro- and macro-mechanical study of spherical granular particles}

Greatness is always built on this foundation: the ability to appear, speak and act, as the most common man.

Hafez

Modelling granular materials can help us to understand their behaviour on the microscopic scale, and to obtain macroscopic continuum relations by a micromacro transition approach. The Discrete Element Method (DEM) is used to investigate the influence of inter -particle friction coefficient and cohesion on the micro and macro behaviour of granular packings in the context of an elasto-plastic contact model. It is shown that the influence of friction coefficient on parameters is more pronounce rather cohesion stiffness. However, the effect of cohesion is not yet negligible. The differences in macro and micro quantities become more pronounced when packings are closer to the jamming point i.e. the lowest density where the system is mechanically stable. Furthermore, we observe that friction and cohesion have an influence on the jamming point for frictional samples. From the microscopic contact characteristics the macroscopic elasticity parameters are determined at different volume fractions. The conventional way to extract elastic constants of 
Micro- and macro-mechanical study of spherical granular particles

a packing is to apply a compression or shear deformation to the entire system. The results show that the stiffness of the packings increases with the volume fraction as expected. Surprisingly, it was observed that elasto-plastic samples experience multiple plastic regimes depending on the applied strain keeping the rate small. An elastic regime for very small strain is followed by the contact plastic regime with reduced bulk moduli; which transits into the structural re-arrangements plastic regime. This interesting intermediate plastic regime is due to the hysteretic contact model with changing contact stiffness during probing of configurations. ${ }^{1}$

\subsection{Introduction}

Granular materials play an important role in many industries, such as pharmaceutical, mining or civil engineering. The macroscopic behaviour of granular material is very different from common solids and fluids. There are different methods to model and understand the macroscopic behaviour of particulate systems. A powerful tool to study granular materials is the Discrete Element Method (DEM) which provides a microscopic insight for the observed behaviour $[41,79,149,150,198,234]$. The contact force model is at the basis of this method [142, 143] and a coupled system of equations is solved to describe the motion of individual particles. Despite the modern computational power, the number of particles that can be simulated is still small compared to reality. This problem can be solved by performing a transition from the micro- to the macro-scale and establishing macroscopic constitutive relations [72]. The microscopic properties can be used to drive macroscopic constitutive relations. These relations are used to describe the particle behaviour on the large scale application/process level [147]. Because of discreteness and the disordered nature of granular materials at the microscopic scale, it is necessary to employ a multi-scale approach which can link the micromechanics of granular systems to the continuum description. The objective of the multi-scale approach is to predict the macroscopic (continuum) constitutive relationship from the microscopic contact constitutive relationship and from appropriate geometrical quantities or state variables by means of suitable averaging techniques.

The main challenge comes when the powders are sticky, cohesive and less flow-able like those relevant in food industry [90]. Research has already been done on cohesive granular materials (see refs [141, 225, 226]), however the influence of cohesion on granular packings is still poorly understood. There are

\footnotetext{
${ }^{1}$ To be submitted: Taghizadeh, K., Luding, S., \& Magnanimo, V. Physical Review E.
} 
two cases where cohesion becomes important. $i$ ) When particles become very small the cohesive forces become larger than the other forces on each particle, as is the case for dry fine powders [142, 250]. $i$ i) Not only the size of the particles contributes to the influence of cohesive attractive forces, but also liquid between the particles does this, as is the case for wet granular materials [58, 169, 205].

The research presented here will focus on dry cohesive and non-cohesive granular particles and DEM is used to study granular packings made of polydisperse particles. The question arises how does the presence of attractive forces affect macroscopic properties of the packings? So far, only a few attempts have been made to answer this question. Gilabert et al. [68] focussed on a twodimensional packing made of particles with short-range interactions (cohesive powders) under weak compaction. Yang et al. [266] studied the effect of cohesion on force structures in a static granular packing by changing particle size. Singh et al. [223] studied the effect of friction and cohesion on anisotropy in granular materials under quasi-static shear. The goal is to understand the influence of the microscopic parameters on the macroscopic properties of the packings. Knowing the influence of cohesion on particulate systems will advance development of new constitutive models to predict the macroscopic material behaviour, to be used to model real life applications and to understand and optimize processes.

Many industrial and geotechnical applications that are crucial for our society involve granular systems at small strain levels. That is the case of structures designed to be far from failure (e.g. shallow foundations or underlying infrastructure), strains in the soil are small and a sound knowledge of the bulk stiffness is essential for the realistic prediction of ground movements [35].

In micromechanical and numerical studies, elastic properties are associated with the deformations of a fixed contact network, and should therefore correspond to the "true elastic" behavior observed in the laboratory for very small strain intervals. Indeed, except in very special situations in which the effects of friction are suppressed and geometric restructuring is reversible, the irreversible changes associated with network alterations or rearrangements preclude all kind of elastic modelling.

The goal here is to focus on the macro-mechanical response of dry frictional packings with elasto-plastic contact model and DEM will be used to study periodic assemblies made of polydisperse spheres. In particular, the paper investigates how inter-particle contact friction and elasto-plastic cohesive contact model influence the bulk response of granular packings [106, 232, 234]. In this work, we analyze the role of the contact model along with microstructure, stress and volume fraction $[121,232,234]$. The ultimate goal is to improve the 
understanding of elasticity in particle systems and to guide the development of constitutive models.

This paper is organized in the following manner. In section 2, the simulation method and parameters used are given. The preparation test procedure and the averaging definitions for scalar and tensorial quantities are explained in section 3. In Section 4, we first explain how the elastic moduli are determined; after that results of small-strain perturbations for different packings are given. Finally, section 5 is devoted to the conclusion remarks and outlooks.

\subsection{Simulation approach}

We use the Discrete Element Method (DEM) to understand the behaviour of granular systems. In the model, we relate the force interacting between the particles to the overlap $\delta$ that the particles have with each other. DEM solves Newton's equations of motion for all forces $\mathbf{f}_{i}=f^{n} \cdot \mathbf{n}+f^{t} \cdot \mathbf{t}$ acting on particle $i$ for the translational and rotational degrees of freedom. The Discrete Element Method (DEM), often referred to as Molecular Dynamics (MD), is a many-particle simulation method. Even though a lot of research verified the usefulness of DEM [207, 255], large scale industrial applications are out of reach. These applications involve even more than the millions of particles that can be simulated using DEM. Instead of simulating real life applications, small samples of representative volume elements (RVEs) can be used to calculate the macroscopic constitutive relations needed to perform the micro-macro transition [149]. Note that the evaluation of the inter-particle forces based on the overlap may not be sufficient to account for the inhomogeneous stress distribution inside the particles.

\subsubsection{Equations of motion}

DEM models the particle interaction by calculating the equations of motion for every particle in the system. This is done for the normal direction, but also for the translational and rotational degrees of freedom. If the forces $f_{i}$ acting on the i-th particle are known and Newton's equations are applied we get [149]:

$$
m_{i} \frac{d^{2}}{d t^{2}} \mathbf{r}_{i}=\mathbf{f}_{i}+m_{i} \boldsymbol{g} \text { and } \quad \mathbf{I}_{i} \frac{d}{d t} \boldsymbol{\omega}_{i}=\mathbf{q}_{i}
$$

where $m_{i}$ is the mass of the $\mathrm{i}$-th particle and $\mathbf{r}_{i}$ the position of the particle. Two types of forces are working on the particles. One force because of the 
interaction with other particles: $\mathbf{f}_{i}=\sum_{c} \mathbf{f}_{i}^{c}$. The other force is due to body forces like gravity $(\boldsymbol{g})$, the particles moment of inertia $\left(\mathbf{I}_{i}\right)$, its angular velocity $\left(\boldsymbol{\omega}_{i}\right)$ and the total torque $\left(\mathbf{q}_{i}=\mathbf{q}_{i}^{\text {friction }}+\mathbf{q}_{i}^{\text {torsion }}+\mathbf{q}_{i}^{\text {rolling }}\right)$.

\subsubsection{Contact model}

For the sake of simplicity, the linear visco-elastic normal contact force model can be used. It involves a linear repulsive and a linear dissipative force: $f^{n}=$ $k \delta+\gamma_{0} \dot{\delta}$ with $k$ as spring stiffness, $\delta=\left(a_{i}+a_{j}\right)-\left(\mathbf{r}_{i}-\mathbf{r}_{j}\right) \cdot \mathbf{n}>0$ as particle overlap, $\mathbf{n}=\mathbf{n}_{i j}=\left(r_{i}-r_{j}\right) /\left|r_{i}-r_{j}\right|$ as normal unit vector, $\gamma_{0}$ as viscous damping coefficient and $\dot{\delta}$ the relative velocity in normal direction $v_{n}=-\mathbf{v}_{i j} \cdot \mathbf{n}=\dot{\delta}$.

An artificial damping force $\mathbf{f}_{b}$ is introduced to reduce dynamic effects and shorten relaxation times: $\mathbf{f}_{b}=-\gamma_{b} \mathbf{v}_{i}$. This will resemble the damping of a background medium, as e.g. a fluid. This force acts not on contacts but directly on particles, proportional to their velocity $\mathbf{v}_{i}$.

Using this model the particle contact can be seen as a damped harmonic oscillator. The advantage is that the half-period of a vibration around an equilibrium position can be computed and so the typical response time on the contact level:

$$
\mathbf{t}_{c}=\frac{\pi}{\omega}, \quad \text { with } \quad \omega=\sqrt{\left(k / m_{i j}\right)-\eta_{0}^{2}},
$$

where $\omega$ is the eigenfrequency of the contact, $\eta_{0}=\gamma_{0} /\left(2 m_{i j}\right)$ the rescaled damping coefficient and $m_{i j}=m_{i} m_{j} /\left(m_{i}+m_{j}\right)$ the reduced mass.

Using the solution of Equation 2.2 the coefficient of restitution is obtained:

$$
r=-v_{n}^{\prime} / v_{n}=\exp \left(-\pi \eta_{0} / \omega\right)=\exp \left(-\eta_{0} t_{c}\right)
$$

which quantifies the ratio of relative velocities after (primed) and before (unprimed) the collision. The integration time-step $\Delta t_{M D}$ used for simulations needs to be much smaller than the contact duration $t_{c}$ to make sure that the integration of the equations of motion is stable. Note that in extreme cases of an overdamped spring, $t_{c}$ can become extremely, artificially large, i.e. dissipation $\gamma$ should be neither too weak nor too strong.

The viscous dissipation mode is suitable for two-particle contact. But when there are a lot of particles involved it becomes very inefficient. Therefore artificial damping is introduced. There will be some additional damping with the background. The background damping is of use for a quick relaxation and the system comes more rapidly to a static equilibrium. The values for the 
Micro- and macro-mechanical study of spherical granular particles

background damping $\left(\gamma_{b}\right.$ and $\left.\gamma_{b r}\right)$ were checked for the used set of parameters to prevent an over-damped system [142].

\subsubsection{Frictional contact model}

Friction is generated when two particles are in contact and have a motion relative to each other. For the simulations presented here a friction model according to the Coulomb friction law is used. This law has two aspects. There is a static friction when two particles do not have micro-slip at the contact surface. In the case of static friction, the friction force between the surfaces of two particles cannot be greater than the product of the normal force $f^{n}$ and the coefficient of static friction $\mu_{s}: f^{t} \leq \mu_{s} f^{n}$. The linear visco-elastic contact model that was introduced earlier is used for the force component in the tangential direction:

$$
f^{t}=k_{t} \delta^{t}+\gamma_{t} \dot{\delta}^{t}
$$

where $k_{t}$ is the tangential stiffness, $\gamma_{t}$ the friction viscosity, $\delta^{t}$ the displacement in the tangential direction and $\dot{\delta}^{t}$ the relative velocity in the tangential direction [142]. The kinetic friction becomes active when the tangential component of the force is exceeding the maximum value of the static force, so when the surfaces of two particles that are in contact start to slide.

For a more detailed description of the force models introduced here and for the rolling and torsional force laws that were used see [142, 149].

\subsubsection{Adhesive, elasto-plastic contact model}

In this work, a linear, hysteretic visco-elastic model is used to describe the interaction between cohesive particles by adding irreversiblity into the linear contact model (see Refs. [142, 223, 224, 254]). This model is a simplified version of the nonlinear hysteretic force laws which were proposed by different authors [242, 243]. In this model, the particles stiffnesses are kept constant with different values during loading and unloading. The contact interaction consists of different phases (see Fig. 2.1). At first, the force increases linearly with the overlap $\delta$ up to $\delta_{\max }$ on the loading (irreversible) branch with slope $k_{1}$. The unloading (reversible) branch starts at $\delta_{\max }$, from where the force decreases with the slope $k_{2}$. The force between two particles becomes zero at overlap $\delta_{0}=\left(1-k_{1} / k_{2}\right) \delta_{\max }$, which represents the plastic contact deformation. The force 
decreases with the same slope $k_{2}$ in the case of further unloading. If the overlap is lower than $\delta_{0}$ during unloading, then an attractive force between particles will be active until the minimum cohesive force branch $f_{\min }$ is reached at overlap $\delta_{\text {min }}=\frac{k_{2}-k_{1}}{k_{2}+k_{c}} \delta_{\text {max }}$. Further unloading leads to the (unstable) attractive force $f^{h y s}=-k_{c} \delta$ on the adhesive branch with the slope $-k_{c}$. If unloading starts at $\delta<\delta_{\max }$, contacts follow branches parallel to the limit value, with a constant unloading stiffness $k_{2}$ until the cohesive branch is reached.

The (hysteretic) force can be written as:

$$
f^{\text {hys }}= \begin{cases}k_{1} \delta & \text { if } k_{2}\left(\delta-\delta_{0}\right) \geq k_{1} \delta \\ k_{2}\left(\delta-\delta_{0}\right) & \text { if } k_{1} \delta>k_{2}\left(\delta-\delta_{0}\right)>-k_{c} \delta \\ -k_{c} \delta & \text { if }-k_{c} \delta \geq k_{2}\left(\delta-\delta_{0}\right)\end{cases}
$$

where $k_{1}, k_{2}$ and $k_{c}$ are contact stiffnesses during loading, unloading and on the adhesive branch, respectively. The contact model presented involves some simplifications with respect to the behaviour observed in experiments, e.g. [230, $242,243,254]$, or proposed by other authors [97, 189, 240]. Among those, it is the piece-wise linear structure, the value of the force at $\delta=0$ and neglecting the detachment of the deformed particles at a finite overlap. A detailed discussion on the model can be found in [224]. Simplifications are mainly driven by case in computation. However, we believe that the influence on the specific aspects studied here is negligible, as our primary focus is on static packings in the small strain regime, where particles detachments/rearrangements are limited.

An overview of the parameters used in the DEM simulations can be seen in table 2.1. The values were examined with two particle collisions (benchmark tests) to validate that the program was working correctly and the linear (hysteretic) force model is correct. The normal force is plotted against the overlap $\delta$ (Fig. 2.2). The hysteretic force diagram had the same characteristics as the theoretical model (Figure 2.1). For different values of the adhesive stiffness $\left(k_{c}\right)$ the adhesive force (the negative force) increases as the $k_{c}$ increases (Fig. 2.2). For the adhesive stiffness values were chosen between a wide range $\left(1 / 20 \leq k_{c} / k \leq 20\right)$ to show the influence well.

\subsubsection{Microscopical quantities}

Here, we define some microscopical quantities that obtained from single contact interaction. These parameters can not usually be measured from experiments, but are easily available from DEM simulations. For single contacts, the contact 


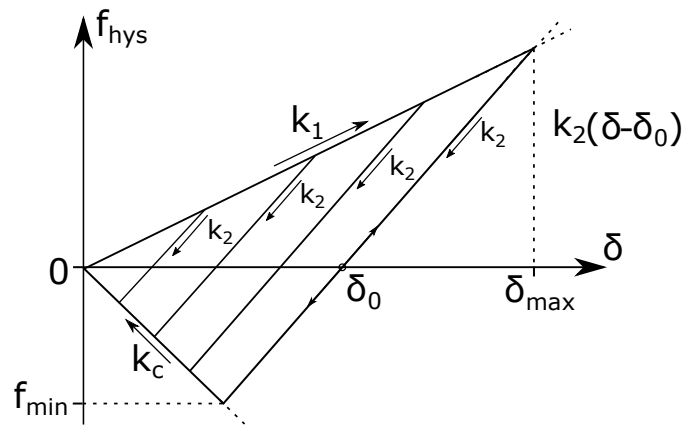

Figure 2.1: Schematic graph of the piece-wise linear, hysteretic model. The adhesive force-displacement for normal collision. The non-contact forces $\left(f_{0}\right)$ are kept equal to zero in this study and also the line for negative $\delta$ is neglected in this paper

\begin{tabular}{llll}
\hline Property & Symbol & Value & SI-units \\
\hline Time unit & $t$ & 1 & $10^{-6} \mathrm{~s}$ \\
Length unit & $x$ & 1 & $10^{-3} \mathrm{~m}$ \\
Mass unit & $m$ & 1 & $10^{-9} \mathrm{~kg}$ \\
Particle radius & $\langle a\rangle$ & 1 & $10^{-3} \mathrm{~m}$ \\
Polydispersity & $a_{\text {max }} / a_{\text {min }}$ & 3 & \\
Number of particles & $N$ & 5000 & \\
Particle density & $\rho$ & 2000 & $2000 \mathrm{~kg} / \mathrm{m}^{3}$ \\
Simulation time step & $\Delta t_{M D}$ & 0.0037 & $3.7 \cdot 10^{-9} \mathrm{~s}$ \\
Unloading (reversible) stiffness & $k_{2}$ & $15 \cdot 10^{4}$ & $15 \cdot 10^{7} \mathrm{~kg} / \mathrm{s}^{2}$ \\
Loading (irreversible) stiffness & $k_{1} / k_{2}$ & 0.666 & \\
Cohesive stiffness & $k_{c} / k_{2}$ & $0-20$ & \\
Tangential stiffness & $k_{t} / k_{2}$ & 0.2866 & \\
Coefficient of friction & $\mu$ & 0.5 & \\
Normal viscosity & $\gamma=\gamma_{n}$ & 1000 & $1 \mathrm{~kg} / \mathrm{s}$ \\
Tangential viscosity & $\gamma_{t} / \gamma$ & 0.2 & \\
Background visc. & $\gamma_{b} / \gamma$ & 0.15 & \\
Backgr. torque visc. & $\gamma_{b r} / \gamma$ & 0.03 & \\
\hline
\end{tabular}

Table 2.1: The microscopic contact model parameters values

force law is reformulated in terms of potential energy density, contact stress, and elastic deformation. Starting from a linear expansion of the interaction potential around static equilibrium, stress can be derived from the principle of virtual displacement. The approach includes both normal and tangential forces. 


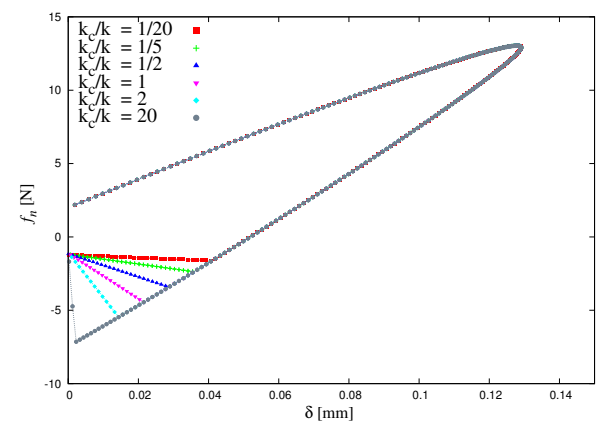

Figure 2.2: Two particles collision in the normal direction using the hysteretic contact model with different cohesive stiffness $k_{c}$. The force in the normal direction is plotted against the overlap $\delta$.

The overlap in normal direction can be expressed as $\vec{\delta}_{n}=\vec{l}-\left(a_{1}+a_{2}\right) \vec{n}$. Where, $a_{i}$ is the radius of a particle, $\vec{l}=\vec{r}_{i}-\vec{r}_{j}$ the branch vector (the difference between particles position), $\vec{n}=\vec{l} / l$ the normal vector with $l=|\vec{l}|=\left(a_{1}+a_{2}\right)$. The normal overlap is the normal deformation relative to the configuration when the particles just get in contact. The total relative deformation can be expressed in a normal and tangential contributions, $\vec{\varepsilon}=\vec{\varepsilon}_{n}+\vec{\varepsilon}_{t}$, which becomes:

$$
\vec{\varepsilon}=\frac{\vec{\delta}_{n}}{l} \vec{n} \vec{n}+\frac{\vec{\delta}_{t}}{l} \vec{t}^{0} \vec{n}
$$

with $\vec{t}^{0}:=\delta_{t} /\left|\delta_{t}\right|$. During the deformation, the length and direction of branch vector, $\vec{l}$, changes. The change of branch vector, $\partial \vec{l}$, can be split into a normal and tangential component as well. The normal component, expressed in index notation ${ }^{2}$, becomes: :

$$
\partial \delta_{\alpha}^{n}=\partial l_{\alpha}^{n}=n_{\alpha} n_{\beta} \varepsilon_{\beta \gamma} l_{\gamma}
$$

and in the tangential component becomes $\partial \vec{\delta}_{t}:=\partial \vec{l}-\partial \vec{l}_{n}$, which can be written as:

$$
\partial \delta_{\alpha}^{t}=\partial l_{\alpha}^{t}=t_{\alpha} t_{\beta} \varepsilon_{\beta \gamma} l_{\gamma}
$$

\footnotetext{
${ }^{2}$ Summation over equal indices is implied.
} 
Hence, the potential energy density for one contact can be expressed in term of the overlap:

$$
u_{c}=\frac{1}{2 V_{c}}\left(k_{n} \vec{\delta}_{n}^{2}+k_{t} \vec{\delta}_{t}^{2}\right)
$$

where, $k_{n}$ and $k_{t}$ are the spring stiffness in the normal and tangential direction, respectively. $V_{c}$ is left unspecified as this volume disappears during averaging, in many cases. The potential energy density changes due to the deformation. The change in the potential energy density can be split into a normal and tangential contribution which results in:

$$
\partial \vec{u}=\partial u_{n}+\partial u_{t} \approx \frac{1}{V_{c}}\left(k_{n} \vec{\delta}_{n} \partial \vec{l}_{n}+k_{t} \vec{\delta}_{t} \partial \vec{l}_{t}\right) \approx \frac{1}{V_{c}} \vec{f}^{*} \cdot \vec{\varepsilon} \cdot \vec{l}
$$

where $\vec{f}^{*}=\left(\vec{f}+\vec{f}^{\prime}\right) / 2$ which is expressed in the actual force, $\vec{f}=k_{n} \vec{\delta}_{n}+k_{t} \vec{\delta}_{t}$, and the force after displacement, $\vec{f}^{\prime}=\vec{f}+\partial \vec{f}$. With the defined potential energy density and deformation, the stress can be derived. By differentiating $u$ with respect to the deformation components:

$$
\sigma_{\alpha \beta}=\frac{\partial u}{\partial \varepsilon_{\alpha \beta}}=\frac{1}{V_{c}} f_{\alpha}^{*} l_{\beta}
$$

Likewise the former terms, the stress term can be expanded into a normal and tangential contributions:

$$
\sigma_{\alpha \beta}=\frac{k_{n} l \delta_{n}}{V_{c}} n_{\alpha} n_{\beta}+\frac{k_{t} l \delta_{t}}{V_{c}} n_{\alpha} t_{\beta}^{0}
$$

which gives the incremental stress tensor as:

$$
\partial \sigma_{\alpha \beta} \approx \frac{k_{n} l \partial \delta_{n}}{V_{c}} n_{\alpha} n_{\beta}+\frac{k_{t} l \partial \delta_{t}}{V_{c}} n_{\alpha} t_{\beta}^{0}
$$

with $\delta_{n}=\left|\vec{\delta}_{n}\right|, \partial \delta_{n}=\left|\partial \delta_{n}\right|, \delta_{t}=\left|\vec{\delta}_{t}\right|, \partial \delta_{t}=\left|\partial \delta_{t}\right|$. 


\subsection{Preparing samples and defining quantities}

In this section, we first describe the preparation procedure of samples and then evolution of quantities during sample preparation are investigated. To create the samples, particles are randomly generated in a 3D-box at very low volume fraction. Each particle is given a small velocity that causes the particles to move, collide and randomize themselves. Particles with cohesion have attractive forces when they separate after interacting, related to the maximal overlap between the particles. If the initially assigned velocities of particles are too large, the particles overlap will become too large (e.g. more than $10 \%$ of particles average radius) when they collide. In such cases, particles will stick to each other which leads to unwanted uncontrolled clusters. Such clusters are not spherical and can cause strong inhomogeneities in the system. Therefore, it is advised that particles are randomized in the 3D-box with sufficiently small velocities and without large initial overlaps to avoid the formation of clusters.

The sample preparation is composed of several steps. At the first step of the preparation process, a loose packing of particles at a volume fraction of $\phi_{0}=$ 0.3 was created, considering the issues explained before regarding unwanted clusters. After random generation, the packing is isotropically compressed to the target volume fraction at $\phi_{1}=0.5$ which is well below the isostatic jamming regime, i.e. the transition from liquid- to solid-like behavior. The system is then relaxed at constant volume fraction $\phi_{1}$ and particles are allowed to dissipate their kinetic energy and to reach zero-pressure. After the relaxation, further isotropic compression is applied up to a volume fraction of $\phi_{\max }=0.82$. In a final phase of preparation the compressed packing are decompressed isotropically from $\phi_{\max }$ to $\phi_{1}$. To obtain an initial isotropic configuration, several driving modes , e.g. wall-driven or strain-rate driven, can be carried out and these modes are discussed in Ref.[92]. As strain-rate driven method produces more homogeneous system, it is more favorable in this study. The compression and decompression are both performed using a constant strain rate applied to each particle which will ensure the homogeneity of the sample.

We use a simulation time of $4000[\mu \mathrm{s}]$ for the first and second phase, which results in a strain rate of $\dot{\varepsilon}=6.6 \cdot 10^{-5}\left[\mathrm{~s}^{-1}\right]$. By following the above procedure frictional and cohesive samples were created by varying the inter-particle friction coeffcient $(0 \leq \mu \leq 1)$ and cohesion stiffness $\left(0 \leq k_{c} / k_{2} \leq 20\right)$. More information about sample preparation of packings can be found in Refs. [106, 234].

Samples with different inter-particle friction and cohesion values were prepared by using the preparation protocol explained before. Note that, the most cohesive particles stick to each other at high volume fractions, which results in 
clusters during the sample decompression; hence, these samples will no longer be homogeneous. For this reason, the results shown later in case of cohesive samples will be taken from the compression path of the sample preparation and those will be relaxed before any further deformation. But, this is not a problem in case of frictional samples, hence, the decompression path is used for further investigations. Below, we discuss the effect of friction coefficient and cohesion between particles on different quantities during the sample preparation.

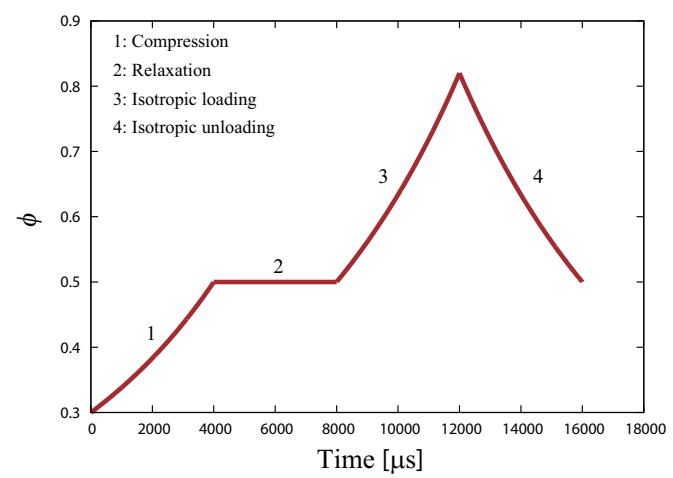

Figure 2.3: Evolution of the volume fraction as a function of the time during sample preparation.

\subsubsection{Jamming transition (from fluid- to solid-like behavior)}

One characteristic these varied systems share is their particular sensitivity to external stress; in densely jammed systems, for instance, the external pressure can cause the system to transition between rigid and floppy states. Jamming governs the transition to rigidity of disordered matter. Granular packing can jam in rigid, disordered states in which it responds essentially elastically to small applied shear stress. However, it can also easily be made to yield (unjam) and flow by tuning various control parameters.

Figs. 2.4a and b show the fractions of particles with a certain number of contacts during compression and decompression. Looking at Fig. 2.4a, there is only a small number of particles having more than three contacts at low volume fraction. It is clear that by increasing the volume fraction, the number of particles with a high number of contacts increases $(\bar{Z} \geq 4)$ and particles with a small number of contacts decreases $(\bar{Z}<4)$. Having a closer look, we can see 
that there is a regime of volume fractions, around the so-called jamming density, where the sharpest changes occur in the number of contacts. This transition regime (around $\phi=0.56$ ) displays in particular a change in slope for $\bar{Z}=4$. The jamming density is the next quantity, it can be obtained from the performed simulations using the pressure and coordination number data. It is defined as the transition of the material from a fluid-like state to a solid-like state. When we look at the pressure this could be seen about the point where the pressure becomes non-zero.

Figure 2.4b shows the particle fractions during the sample decompression. As expected, the number of particles with high numbers of contacts is greater at high volume fraction. By moving towards the decompression path, it can be seen that there is not a significant change in the number of particles with high number of contacts (especially for $\bar{Z}=4,5,6$ ); this proves the forming of agglomerates during decompression due to the cohesive forces. Particles stuck together will be attached to each other during decompression due to the high cohesive force among themselves. Note that the compression path is used for further results, because decompression path could not be used due to inhomogeneity as mentioned earlier.

\subsubsection{Macro-quantities during the sample preparation}

Here, we present the general definition of the investigated quantities and the results obtained during the sample compression for samples prepared with different friction coefficient and cohesive stiffness.

\section{Coordination number}

Coordination number was calculated during sample compression to observe the influence of cohesion and friction. The coordination number is defined as the average number of contacts per particle $(Z=M / N$, where $M$ is the total number of contacts and $N$ is the total number particles). Particles with zero number of contacts and particles having a too small number of contacts, so called rattlers, were excluded, because they do not contribute to the mechanical stability of the packing [73]. To identify the rattlers, the contacts of the particles are counted. If the number of contacts is less than four, the particle is considered mechanically not stable and is defined as rattler. So the coordination number becomes: $\bar{Z}=$ $M_{3} / N_{3}$, with $M_{3}=$ total number of contacts of particles with at least 3 contacts and $N_{3}=$ number of particles with at least 3 contacts. 


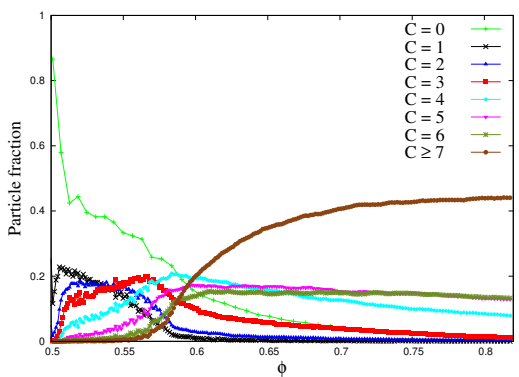

(a)

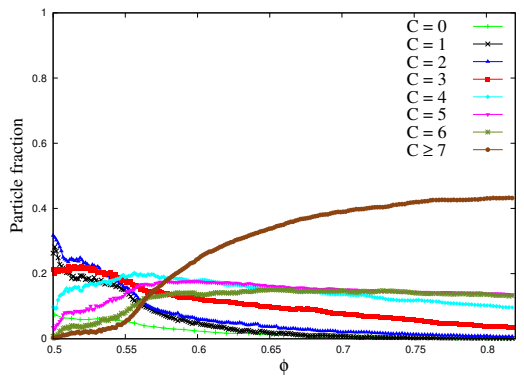

(c)

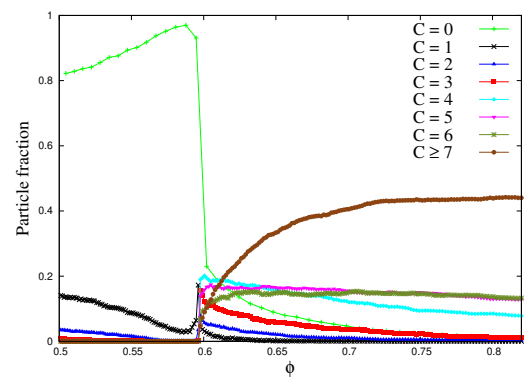

(b)

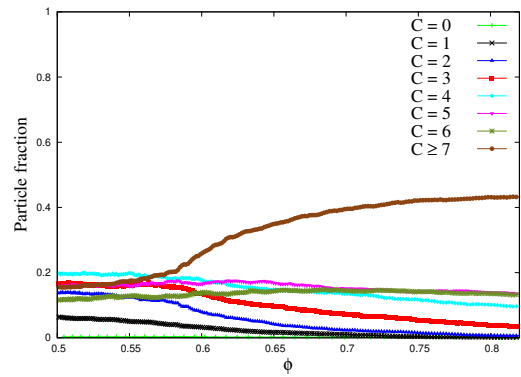

(d)

Figure 2.4: Fraction of particles carrying number of contacts from 0 to 6 or more, for the frictional sample $\mu=0.5$ with $k_{c} / k=0$ (a,b) and $k_{c} / k=10(\mathrm{c}, \mathrm{d})$, during the sample compression (left pictures) and decompression (right pictures).

Fig. 2.5 shows the evolution of the coordination number as function of $\phi$ for samples frictional and cohesional samples. Looking at Fig. 2.5.a, one can see the effect of friction coefficient is crucial on the coordination number where increasing friction coefficient at dense regime leads to a lower coordination number.Also, as the particle friction increases, the critical jamming fraction shifts towards the left side of $x$-axis. Our observation is consistent with the previous study Refs. [73, 74].

Fig. 2.5.b depicts the behaviour of coordination number with respect to the cohesive stiffness. For the lower densities, below jamming, a systematic increase in $\bar{Z}$ can be seen with respect to $k_{c}$. This is less pronounced for highly dense samples. As we know, cohesive forces are more active at low volume fractions, therefore the change of coordination number at low volume fraction can be 


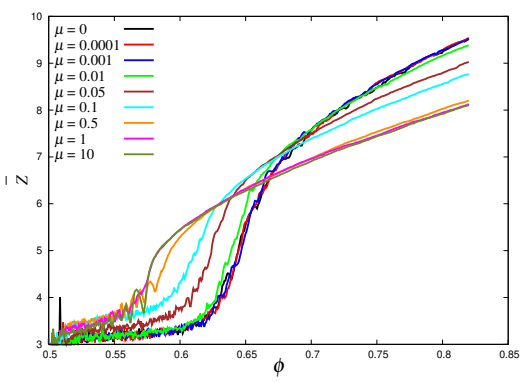

(a)

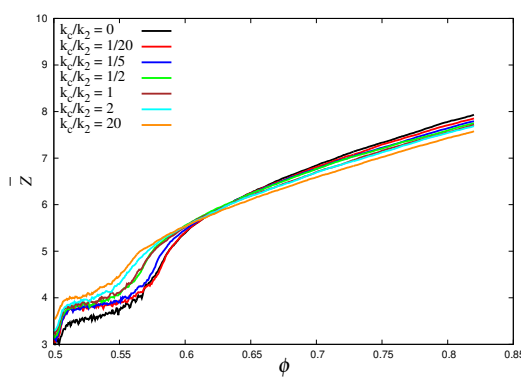

(b)

Figure 2.5: Coordination number plotted against volume fraction $\phi$ for different (a) friction coefficient $\mu$ and (b) cohesion values $k_{c}$, during compression (solid lines with points).

observed. By looking at the coordination number plots, it can be understood that the effect of cohesive forces is more important at lose packings (close to the jamming density) rather to dense packings. This is due to activation of cohesive forces close to the jamming density since particles have more space to attract and repel each other. While going far from the jamming density, there is not enough space for particles to establish cohesive forces since they are fully compressed and their normal force is dominated by elastic loading branch.

\section{Pressure}

The stress tensor is a macroscopic quantity that can be obtained by measurement of forces per area, or via the averaging procedure. One can obtain the approximate macroscopic stress by averaging over the volume $V$ using the stress computed earlier (Sec. 2.2.5) at the contact level:

$$
\sigma_{\alpha \beta}=\frac{1}{V} \sum_{p \in V} V^{p} \sigma^{p}=\frac{1}{V} \sum_{p \in V} \sum_{c=1}^{C^{p}} l_{\alpha}^{c} \otimes f_{\beta}^{c}
$$

which is an average over the contacts in the volume $V$ of the dyadic products between the branch vector $\mathbf{l}^{c}$ and the contact force $\mathbf{f}^{c}$, where the contribution of the dynamics has been neglected. Note that the particle volumes cancel due to the volume weight. The isotropic component of the stress is the pressure. We 
use the dimensionless pressure which is calculated by using the average normal stress: $P^{*}=P / k^{*}$, where $P=\left(\sigma_{x x}+\sigma_{y y}+\sigma_{z z}\right) / 3$ and $k^{*}=k_{1} /(2\langle a\rangle)$.

Note, we avoid using $*$ sign for the data shown later. The dimensionless pressure $(P)$ is plotted in Fig. 2.6 for isotropic compression from $\phi_{\max }=0.5$ to $\phi_{0}=0.82$ (isotropic loading path in Fig. 2.3) for samples prepared with different friction coefficients and cohesion. It is not surprising to see the increase in the pressure with volume fraction since the samples get more compacted. On the other hand, for a given volume fraction, the pressure increases with the coefficient of friction and cohesion, and the jamming volume fraction $\phi_{c}^{*}$ decreases with friction and cohesion.

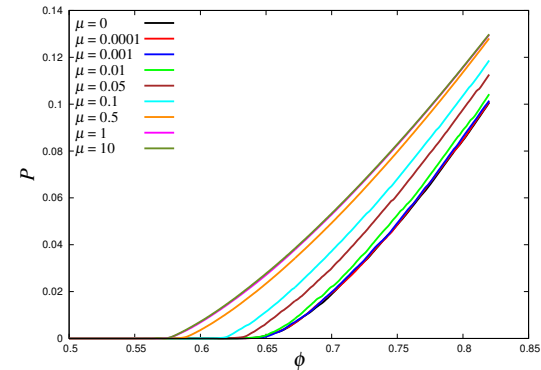

(a)

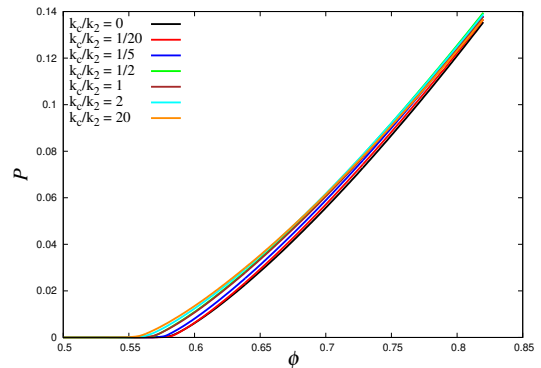

(b)

Figure 2.6: Dimensionless pressure $P$ plotted against volume fraction $\phi$ for different (a) friction coefficients $\mu$, and (b) cohesion values $k_{c}$, during compression.

\section{Energy ratio}

The ratio of kinetic energy and potential energy $\left(E_{k i n} / E_{\text {pot }}\right)$ are plotted in Fig. 2.7 for isotropic loading path from $\phi_{0}=0.5$ to $\phi_{\max }=0.82$. As increase of friction coefficient causes to a higher tangential component of force which decreases the mobility of particles (less kinetic energy), we can see the decrease of energy ratio at a given volume fraction with friction coefficient by looking at the energy ratio plots. Similar observation is obtained when cohesive stiffness is active since the attractive forces lead to less mobility of particles, thus lower kinetic energy. Likewise the pressure plot, the jamming transition point is moved to the left on volume fraction axis by increasing the friction coefficient. 


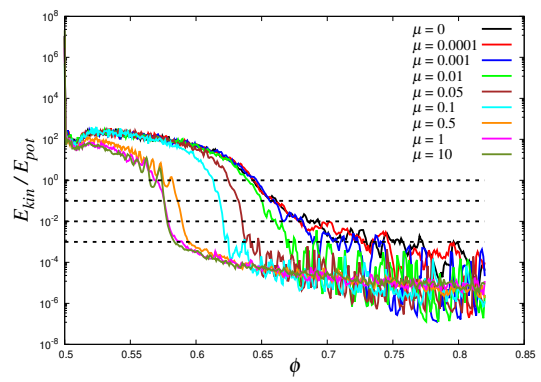

(a)

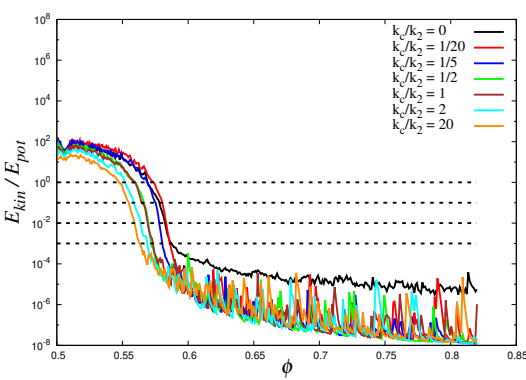

(b)

Figure 2.7: Energy ratio $E_{k i n} / E_{\text {pot }}$ plotted against volume fraction $\phi$ for different (a) friction coefficients $\mu$, and (b) cohesion values $k_{c}$, during compression.

\section{Transition from flow to quasi-static (inertial number)}

As it was mentioned earlier, one of the most fascinating properties of granular materials is their ability to show both solid- and fluid-like behavior. In the absence of a unified framework, granular flows can be classified into three different regimes: quasi-static, inertial (dense flow) and rapid flow. $(i)$ : The quasistatic behavior of granular materials occurs at low applied rate where particles remains in contact and their interactions with their neighbors happen over long periods of time. $(\mathrm{i} i$ ): Increasing the deformation rate causes a transition from quasi-static to dense flow of granular materials where the material flows more as a liquid and particles still face multi-contact interactions but partners change frequently. The transition regime from these two regimes is the jamming transition where flow stops. ( $i$ i ): Beyond the fluid-like flow regime, granular media experience gas-like behavior at very high velocity. In this regime, particles interact through binary since the duration of their interactions is shorter than the time between contacts $[55,88]$.

The volumetric inertial number $I_{\nu}=\dot{\varepsilon}\langle d\rangle \sqrt{\mathbb{D} / P}$ is a dimensionless value that can be used to identify the transition between regimes (i) and (ii). Here, $\dot{\varepsilon}$ is the strain rate (volumetric and deviatoric in case of isotropic and deviatoric deformation, respectively), $\langle d\rangle$ is the mean particle diameter, $P$ is the confining pressure, and $\mathbb{D}$ is the particle density $[65,102]$. The inertial number is the ratio between the microscopic inertial time scale $\langle d\rangle \sqrt{\mathbb{D} / P}$ (also referred to as particle relaxation time) and the macroscopic time scale associated with the applied rate $1 / 3 \dot{\varepsilon}_{v o l}$. The flow goes to the quasi-static limit for low values of $I_{\nu}<0.001$, which 
Micro- and macro-mechanical study of spherical granular particles

is associated to a dense network of enduring contacts. Upon increasing $I_{\nu}$, the transition between the quasi-static and inertial flow takes place in the range of $0.001<I_{\nu}<0.01$. The inertial value between $0.01<I_{\nu}<1$ corresponds to the intermediate fluid-like regime where the dynamical inertial effects have crucial impact. The inertial number of granular assemblies with different friction coefficient during isotropic compression and decompression of sample preparation (path 3 and 4) were calculated (data not shown). It was found that by increasing the volume fraction $\phi$, the inertial number saturates between $10^{-4}$ and $10^{-5}$ in the quasi-static regime and the samples are rate independent in this regime. As we want to explore the elastic behavior of solid-like granular samples, we focus on the regime of small $I_{v}$ (smaller than 0.0001) in this research, where we are sure that the granular sample is in the quasi-static regime.

The inertial number of granular assemblies with different friction coefficient and cohesion during isotropic compression (path 3 in Fig. 2.3) are illustrated in Fig. 2.8. It is clear that by increasing the volume fraction $\phi$, the inertial number saturates between $10^{-4}$ and $10^{-5}$ where is the quasi-static regime and the sample is rate independent in this regime. Moving towards the left side of volume fraction axis, the assemblies experience a transition $\left(10^{-4}<I_{\nu}<10^{-2}\right)$ which is the jamming regime explained earlier. Also, it is can be found that when the samples are loose $\left(\phi<\phi_{c}\right)$, the inertial number varies a lot due to the existence of dynamical collisions between grains. Likewise the other quantities, the effect of the particle friction on the inertial number is more pronounced than the cohesion.

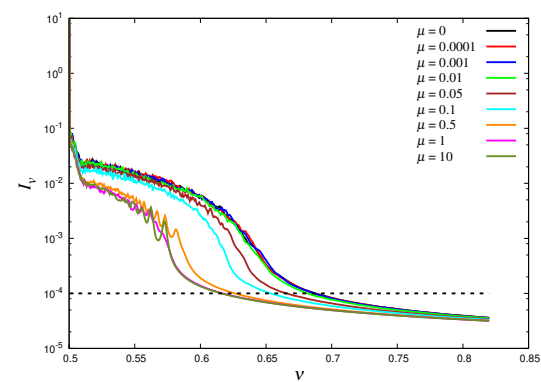

(a)

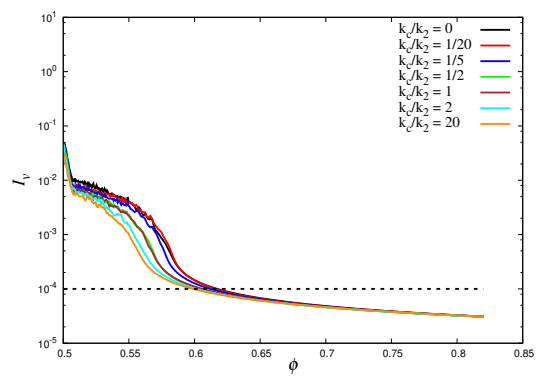

(b)

Figure 2.8: Inertial number $I_{v}$ plotted against volume fraction $\phi$ for different (a) friction coefficients $\mu$, and (b) cohesion values $k_{c}$, during compression. 


\subsection{Small strain stiffness}

Even though millions of particles can be simulated, the possible length of such a particle system is in general too small in order to regard it as macroscopic. These microscopic quantities are used to derive macroscopic quantities needed to describe the material within the framework of a macroscopic continuum theory. The microscopic quantities obtained from DEM simulations (e.g. force, position, and etc) can be reformulated and averaged to derive the macroscopic quantities. These macroscopic quantities give an impression of the whole packing. Therefore, can give an insight into the behaviour of the packing.

We now discuss the conditions for which the response to load increments of a pre-stressed granular packing in mechanical equilibrium can be described as elastic, and explain how macroscopic elastic moduli are computed in our simulations. Furthermore, we show results for larger strain deformations beyond the elastic regime.

Several configurations are chosen with different volume fractions $\phi_{i}$, above the jamming regime, from the loading branch of the preparation path for samples prepared with cohesion and cohesionless. A sufficient relaxation period is applied at constant volume fraction to allow the particles to fully dissipate their energy and to reach equilibrium. From the kinetic to potential energy ratio we conclude that systems with $\phi_{i}>\phi_{j}$ (where $\phi_{j}$ is the material- and procedure-dependent, jamming density [122]) reach a static state as soon as the energy ratio (kinetic to potential) drops well below $10^{-8}$, as confirmed from many additional simulations (data not shown). We assume that the packing is in equilibrium when the ratio of kinetic to potential energy is less than $10^{-8}$ or when the inertial number is lower than $10^{-4}$.

These relaxed configurations can now be used to study the effective stiffness of the granular assemblies. The stiffness is measured by applying strain to the sample in a given direction $\varepsilon_{i j}$ and measuring the resultant change in stress $\sigma_{i j}$ [114]. Due to the preparation procedure, samples can be considered isotropic, where by definition the material properties are independent of direction within random fluctuations (data not shown). Such materials are fully characterized by only 2 independent elastic constants. The two elastic constants are usually expressed as the Young's modulus $E$ and the Poisson's ratio $v$. However, the alternative elastic constants $K$ (bulk modulus) and $G$ (shear modulus) can be directly measured as shown next, and are linked to $E$ and $v(K=E /(3-6 v)$ and $G=E /(2+2 v))$.

In particular, the bulk and shear stiffness of isotropic samples, $K$ and $G$, are calculated by means of isotropic and deviatoric strains respectively. Isotropic 
Micro- and macro-mechanical study of spherical granular particles

compression of samples is such that samples are homogeneously compressed along all directions; during pure shear, samples are compressed along the $x$ direction and decompressed along the $y$-direction, while the $z$-direction is kept stationary. Shear deformations are applied in the form of a pure shear, i.e. by having a displacement in the $y$-direction imposed on all particles that cross the $x$-boundary and a displacement in the $x$ direction on all particles that cross the $y$-boundary. One can obtain the samples bulk $(K)$ and shear $(G)$ moduli, by measuring to incremental response of isotropic and shear deformation:

$$
K=\left.\frac{\delta P}{\delta \varepsilon_{\nu}}\right|_{\delta \varepsilon_{d e v}=0} \quad \& \quad 2 G_{x y}=\left.\frac{\delta\left(\sigma_{x x}-\sigma_{y y}\right)}{\delta\left(\varepsilon_{x x}-\varepsilon_{y y}\right)}\right|_{\delta \varepsilon_{v}=0}
$$

where $P$ is the hydrostatic pressure, $\sigma_{i j}$ is the static stress tensor, and $\varepsilon$ is the applied strain with $\delta \varepsilon_{v}=3 \delta \varepsilon_{v o l}=\delta\left(\varepsilon_{x x}+\varepsilon_{y y}+\varepsilon_{z z}\right)$ and $\delta \varepsilon_{d e v}=\delta\left(\varepsilon_{x x}-\varepsilon_{y y}\right)$ terms. Note that stress and moduli have been normalized by the loading stiffness and mean radius $k^{*}=k_{1} /(2\langle a\rangle)$.

Since we apply small but finite strain perturbations, we do not want slippage to occur; therefore the friction coefficient is set to infinity $(\mu=\infty$, i.e. a large value) to avoid sliding of contacts during probing of samples. For each calculation, we verify that the applied strain is small enough to be in the linear, elastic, reversible regime where the coordination number does not vary significantly during the applied increment of deformation. For each configuration, we apply an identical strain-rate value $3 \dot{\varepsilon}_{v o l}=\dot{\varepsilon}_{x x}+\dot{\varepsilon}_{y y}+\dot{\varepsilon}_{z z}=3.10^{-6}$ and $\dot{\varepsilon}_{d e v}=\dot{\varepsilon}_{x x}-\dot{\varepsilon}_{y y}=2.10^{-6}[1 / \mathrm{s}]$ to assure that the mechanical response of assemblies is in the elastic regime. The inertial number of granular assemblies with during isotropic and shear probing is between $10^{-4}$ (samples with low volume fraction) and $10^{-6}$ (samples with high volume fraction), which is the quasi-static regime and the sample is rate independent in this regime. In the following, we first describe the elastic behavior of frictional samples, then, we investigate the phase transition, from elastic to plastic, of samples prepared and probed with cohesion (i.e. elasto-plastic contact model employed).

\subsubsection{Incremental response of frictional samples}

As we are interested in the elastic moduli, we have to identify the transition from the linear elastic to the non-linear and plastic regime. First, we consider samples having the same friction coefficient at different confining pressures, and we explore the elastic regime of granular samples. Next, we discuss about the reversible and irreversible behaviour during sample probing. Finally, we 
compare the results obtained by isotropic and deviatoric probing with uniaxial probing to show the consistency with continuum mechanics (isotropic linear elasticity) for particulate systems.

\section{Elastic regimes identification}

In order to show the transition from elastic to plastic regime, the bulk and shear modulus with various amplitudes of the applied isotropic, $3 \delta \varepsilon_{v o l}$, and deviatoric strain, $\delta \varepsilon_{d e v}$, during probing are depicted for chosen configurations at confining pressure levels ( $P=0.01,0.03,0.06$ and 0.09 ), and two friction coefficients ( $\mu=0.0001$ and $\mu=1$ ), in Figs. 2.9 and 2.10. The elastic moduli stay practically constant for small amplitudes, $\delta \varepsilon_{v o l}$ and $\delta \varepsilon_{d e v}<10^{-3}$. We assume this regime to be elastic. By increasing the amplitudes of the perturbation, $K$ and $G$ start to increase and decrease, respectively.

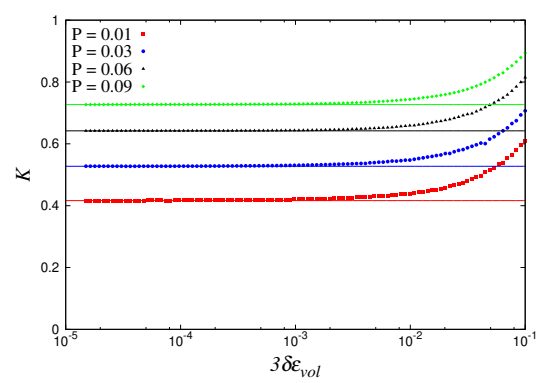

(a)

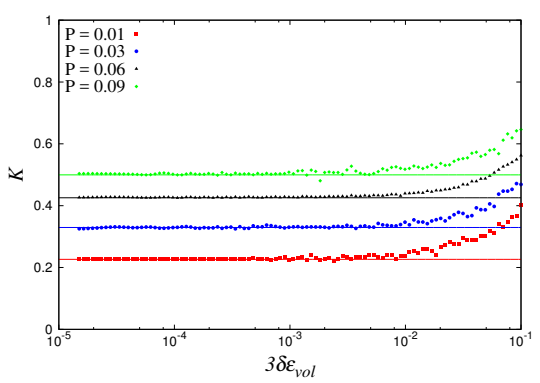

(b)

Figure 2.9: Evolution of bulk modulus $K$ with applied isotropic $3 \delta \varepsilon_{v o l}$ strain for different states at different confining pressure stages $P=0.01,0.03,0.06$ and 0.09 , with different friction coefficients: (a) $\mu=0.0001$ and (b) $\mu=1$ probed with $\mu=\infty$. Corresponding solid lines represent the small strain limit value of $K$ (elastic bulk modulus).

The second order symmetric fabric tensor $\mathbf{F}$ provides information on the spatial distribution of the contacts through its eigenvalues and it is computed as:

$$
\mathbf{F}=\frac{1}{V} \sum_{\mathscr{P} \in V} V^{\mathscr{P}} \sum_{c \in \mathscr{P}} \mathbf{n}^{c} \otimes \mathbf{n}^{c}
$$

which is weighted according to $V^{\mathscr{P}}$, the particle volume of particle $\mathscr{P}$, for all 


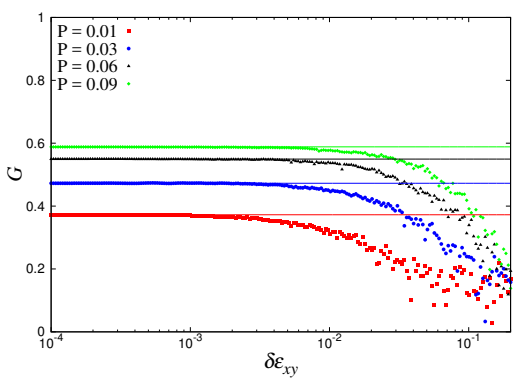

(a)

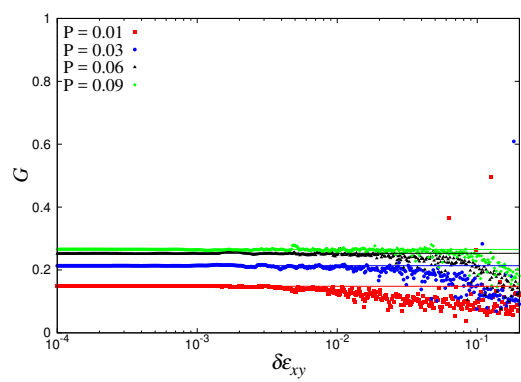

(b)

Figure 2.10: Evolution of shear modulus $G$ with applied deviatoric $\delta \varepsilon_{x y}$ strain amplitudes for different states at different confining pressure stages $P=0.01$, $0.03,0.06$ and 0.09 , with different friction coefficients: (a) $\mu=0.0001$ and (b) $\mu=1$ probed with $\mu=\infty$. Corresponding solid lines represent the small strain limit value of $G$ (elastic shear modulus).

particles inside the averaging volume $V$, with the normal unit branch-vector $\mathbf{n}^{c}$ pointing from the center of particle $\mathscr{P}$ to contact $c$.

To get a better understanding, we study the change of microstructure. Figs. 2.11 and 2.12 show the change of volumetric fabric $\left(F_{\nu}=F_{x x}+F_{y y}+F_{z z}\right)$ and deviatoric fabric $\left(F_{d e v}=F_{x x}-F_{y y}\right)$ for the same configurations as presented before. To make the change visible, the initial values of fabric (their elastic regime, $F_{v}^{\circ}$ and $F_{x y}^{\circ}$ ) was subtracted. Looking at Figs. 2.11 and 2.12, it can be seen that the microstructure does not change for small strains, which ensures that packings are in the elastic regime.

When we apply more strain, we can see that the microstructure starts to increase (dramatically) which reveals that packings large deformation. Comparing Figs. 2.9 and 2.10 with Figs. 2.11 and 2.12, shows that non-linearity of elastic moduli is associated with considerable changes of the microstructure. Fig. 2.9 to 2.12 show that the elastic regime gets wider when the confining pressure gets higher, since samples are more compacted and particles have limited space to rearrange. Therefore, to move particles at high confining pressure, more strain must be applied to destruct the contact network. 


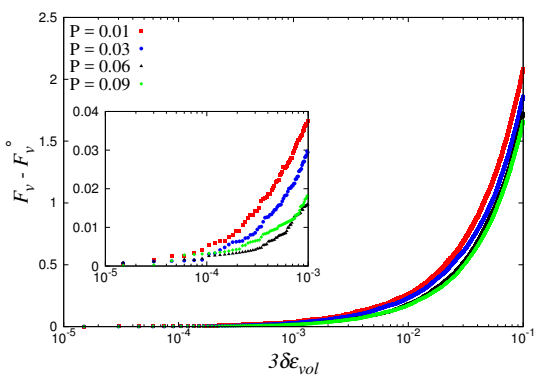

(a)

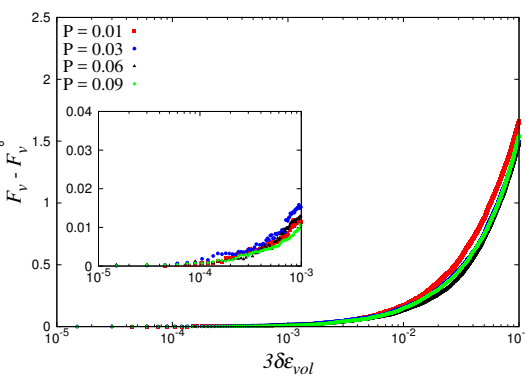

(b)

Figure 2.11: Evolution of isotropic fabric $F_{v}$ with the respective applied isotropic $\delta \varepsilon_{v}$ strain amplitudes for different states at different confining pressure stages $P=0.01,0.03,0.06$ and 0.09 , with different friction coefficients: (a) $\mu=$ 0.0001 and (b) $\mu=1$. Note that the $x$-axis is log-scale.

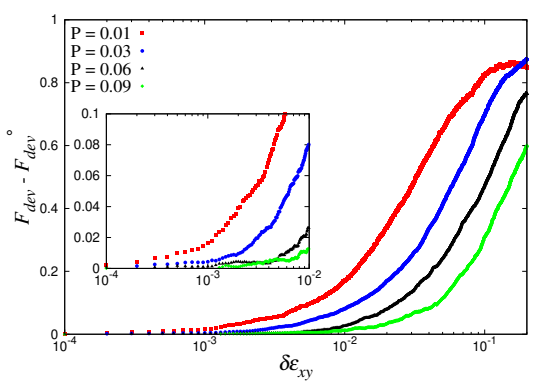

(a)

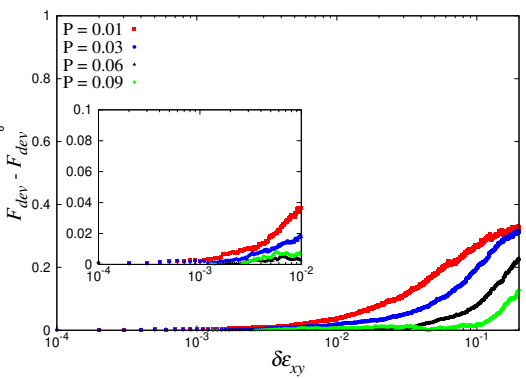

(b)

Figure 2.12: Evolution of deviatoric fabric $F_{x y}$ with the respective applied deviatoric $\delta \varepsilon_{x y}$ strain amplitudes for different states at different confining pressure stages $P=0.01,0.03,0.06$ and 0.09 , with different friction coefficients: (a) $\mu=0.0001$ and (b) $\mu=1$. Note that the $x$-axis is log-scale.

\subsubsection{Reversibility - from elastic to plastic}

By definition, elasticity implies reversibility. To confirm the reversibility of the elastic regimes shown in Figs. 2.9 and 2.10, we tested those samples by reversing strain, i.e. at different levels in Figs. 2.9 and 2.10, where the elastic moduli have different values. We first applied enough relaxation to make sure that 
the energy ratio remains well below $10^{-10}$ and thus coordination number does not dynamically change (fluctuate). After the relaxation, small strain perturbations are applied to configurations in the opposite direction with respect to the previous strain for both isotropic and sheared samples. Figs. 2.13 and 2.14 show the elastic moduli of chosen configurations at pressure level of $P=0.06$, for friction coefficients $\mu=0.0001$ and 1 , during loading and unloading path. The moduli of loading and unloading are identical when the strain is reversed at very small previous deformation level. This confirms reversibility (blue data points in Figs. 2.13 and 2.14). However, when the load is reversed after large previous strain, the moduli show different values which confirms irreversibility and plasticity.

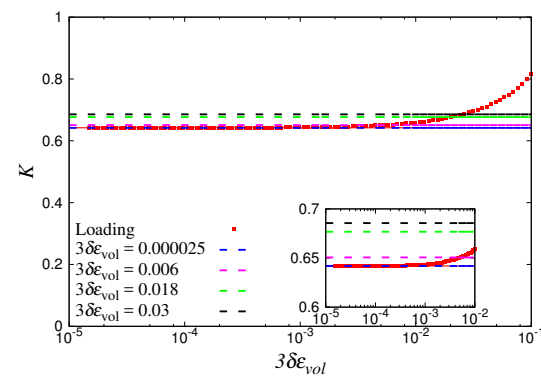

(a)

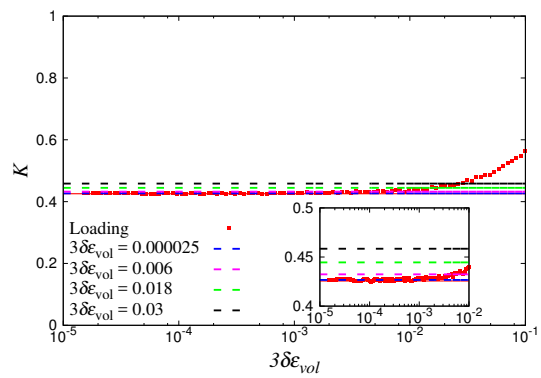

(b)

Figure 2.13: Loading (red points) and unloading path of the bulk modulus $K$ for configurations at $P=0.06$ with friction coefficients of (a) $\mu=0.0001$ and (b) 1 . Dashed lines correspond to the bulk modulus obtained during unloading the samples after different strain amplitudes as given in the legend.

\section{Uniaxial probing, $M$-modulus}

Although only 2 independent elastic constants ( $K$ and $G$ ) are required to describe the elastic stiffness of an isotropic elastic medium, there are many different constants from which to choose. Most of these constants arise as constants of proportionality between stress and strain for various loading conditions. The longitudinal modulus $M$, also known as the P-wave modulus or constrained modulus, is one of the constants that can be measured under certain other loading conditions and by new methods such as wave propagations [156, 172, 173, 234]. The physical meaning of $M$ is the stiffness for tension or compression in 


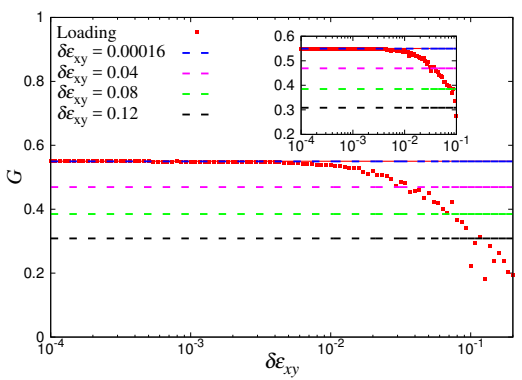

(a)

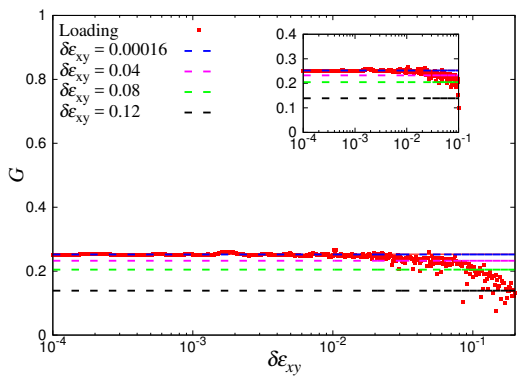

(b)

Figure 2.14: Loading (red points) and unloading path of the shear modulus $G$ for configurations at $P=0.06$ with friction coefficients of (a) $\mu=0.0001$ and (b) 1 . Dashed lines correspond to the shear modulus obtained during unloading the samples after different strain amplitudes as given in the legend.

one direction, when the strain in the other two directions is constrained to be zero. General continuum mechanics says that there is a one-to-one relation between different elastic constants for an isotropic body, so that the longitudinal modulus $M$ can be obtained from bulk and shear modulus as $M=K+(4 / 3) G$.

In order to confirm the validity of this relation for our particulate systems, we apply uniaxial deformations (along the $x$-direction) on configurations chosen before in Sec. 2.4.1. Uniaxial probing is an other deformation mode, superposing isotropic and deviatoric, which gives us the longitudinal modulus along the probed direction. In Fig. 2.15, we plot the longitudinal modulus $M$ obtained by direct (uniaxial probing) and indirect (isotropic and deviatoric probing) measurement. This figure clearly states that the results obtained by two different simulation approaches well coincide, which confirms the validity of continuum mechanics assumptions for the case of our isotropic granular materials as long as they are in the elastic regime. For that reason, once can compute the bulk and shear moduli from uniaxial test (data not shown) in agreement with direct testing.

\subsubsection{Incremental response of cohesive samples}

In this subsection, we visit to represent the influence of cohesion between particles on the elastic moduli of cohesional packings. Results of the overall stiffness in the case of cohesive samples is shown in Fig 2.16. Samples prepared with 


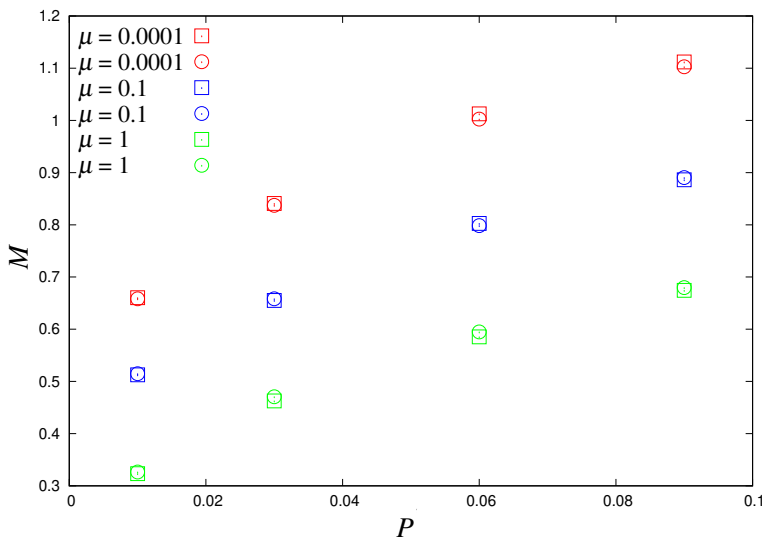

Figure 2.15: Longitudinal modulus $M$ obtained by two different approaches for samples with different friction coefficients $\mu=0.0001,0.1$ and 1 at different confining pressure level. 'o' and ' $\square$ ' represent values of $M$ measured by direct uniaxial probing and indirect determination $\left(K+\frac{4}{3} G\right)$, respectively.

internal contact elasto-plasticity and cohesion (Fig. 2.1) show a different, more complex behavior as shown in Fig. 2.16. Similar to cohesionless materials, the stiffness stay constant at very small applied strain level $\left(3 \varepsilon_{v o l}\right.$ and $\left.\varepsilon_{d e v} \leq 10^{-5}\right)$. Increasing the applied strain in both isotropic and shear modes leads to the transition from the initial elastic regime to a second plateau, where the stiffness is again constant, but assumes a different value with respect to the very small strain regime. As the effective stiffness is constant, this must be read as a second elastic regime. Finally, when large strain is applied to the system rearrangement happens. We associate the transition from the first to the second elastic regimes to the contact model.

As explained earlier, configurations have been chosen from the compressional branch (where most contacts have loading stiffness $k_{1}$ ) and relaxed before probing. During the relaxation, contacts move from the loading branch of the hysteretic contact model to the unloading branch (with stiffness $k_{2}$, see the arrows in Fig. 2.1). This means that pair-particle contacts will obtain the stiffness of the elastic branch at the end of relaxation. Therefore, when probing starts, the elastic stiffness of the samples is controlled by the contact stiffness $k_{2}$. However, when larger strain is applied, many contacts transit from the un/reloading (reversible) branch $k_{2}$ to the loading (irreversible) branch $k_{1}$. 
This transition at the contact level leads to a reduction of the bulk stiffness, with elastic moduli at very small strain 1.5 larger than the second regime in agreement with the ratio $k_{1} / k_{2}=0.66$.

To summarize, we have identified three regimes for the stiffness of cohesive granular packings: (i) a first elastic regime dominated by the unloading branch of the contact model at very small strain, (ii) a second (pseudo) elastic regime dominated by the loading branch of the contact model at moderate strain, and (iii) a plastic regime associated to large structural rearrangements of particles. The increase (decrease) of bulk (shear) modulus at larger strain is accompanied by large fluctuations due to permanent rearrangements. Note that the magnitude of cohesion (value of $k_{c}$ ) has very little effect on $K$ and $G$ (data not shown).

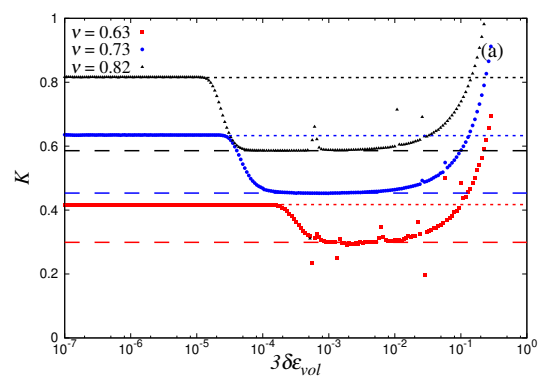

(a)

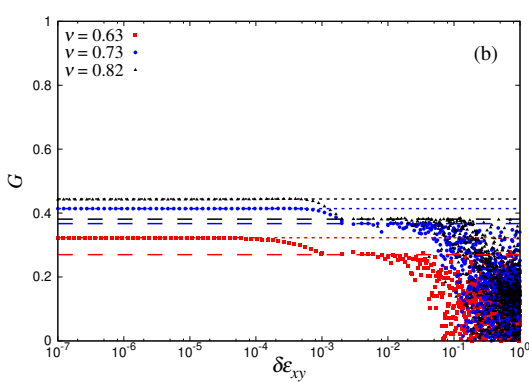

(b)

Figure 2.16: Normalized (a) bulk modulus $K$ plotted against volumetric strain and (b) shear modulus $G$ plotted against deviatoric strain at different volume fractions for elasto-plastic cohesive $k_{c} / k_{2}=1$ granular samples. Dotted lines correspond to the elastic regime at very small strain with contact stiffness of $k_{2}$. Dashed lines correspond to the elastic regime of packings with reduced stiffness with the ratio $k_{1} / k_{2}=0.66$.

\subsection{Concluding remarks}

In this study the influence of friction and cohesion on the macro- and microscopic properties was observed. Frictional samples were prepared with and without inter-particle cohesion. Sample preparation plays a key role to obtain a homogeneous medium; for more cohesive packings we observed stronger 
Micro- and macro-mechanical study of spherical granular particles

formation of clusters and agglomerates during sample preparation in particular during unloading. During compression, the influence of cohesion is more pronounced for samples due to tangential forces that cause rotations and rearrangements. Moreover, cohesion and friction both play a more significant role at low volume fractions, since samples have not been compacted yet and translational and rotational movement of particles can activate forces. The jamming density is a crucial state variable at which the transition from fluid to solid-like behaviour of configurations occurs.

In the second part of this paper, the influence of contact model details, such as elasto-plasticity (reversibility vs. irreversibility at the contact level), on the macroscopic stiffness of granular materials was investigated. Configurations at different volume fractions were chosen and relaxed to reach mechanical equilibrium. Then, we applied different deformation modes to obtain the bulk and shear stiffness of the packings. The behavior of cohesive samples is more complex than for particles with a linear contact law due to the existence of forces between the particles, where the attractive forces are only playing an important role for large strain tensile or shear probing (data not shown). The transition from the elastic to the plastic regime of irreversible samples is not as continuous as for linear samples. It was found that particles will settle on the unloading branch of the triangular contact model at the end of relaxation before probing. When probing the samples, the stiffness of the contacts will start to change from unloading to loading, which was the reason of the transition of the elastic moduli at small strain level. After the stiffness transition at the contact points, elastoplastic samples followed the behavior of the linear non-cohesive ones with the same stiffness $k_{l i n}=k_{1}$. Applying more strain caused a second meso-scopic level of irreversible behaviour of the packings due to structural rearrangements.

An interesting perspective is to use experimental measurements of the stiffness at different strain/probing amplitudes to infer useful information on the inner structure and the contact mechanics of granular packings. That, in turn, can be exploited to predict their behavior under large deformations. 


\section{Chapter 3}

\section{Micromechanical study of the elastic stiffness in isotropic frictional granular solids}

To wisely live your life, you don't need to know much; Just rememeber two main rules for the beginning: You better starve, than eat whatever And better be alone, than with whoever.

Omar Khayyam

Understanding the pre-failure, elastic behavior of dense granular systems is of interest in many fields, such as soil mechanics, process engineering, material science and physics. The Discrete Element Method (DEM) allows to inspect the influence of microscopic contact properties of its individual constituents on the macro bulk behavior of granular assemblies. In this study we use DEM to explore the elastic response of frictional granular materials. Small, isotropic and deviatoric deformations ("probes") are applied to isotropic packings of frictional spheres featuring both linear and non-linear normal interactions; with different inter-particle contact friction coefficients. At various pressure levels, the effective elastic moduli are determined from the incremental stress response to the applied strain. For both types of contact interactions, the moduli exhibit a non-trivial dependence on the preparation procedure. With increasing inter-particle contact 
friction during preparation, the moduli decrease for samples with the same volume fraction, but a much larger stress at maximal overcompression during preparation. We explain this by differences in the microstructure (decreasing coordination number for increasing friction) that characterises the sample state after preparation. Major differences in magnitude appear in the tangential force contributions to the shear modulus. The systematic, unexpectedly small moduli due to tangential forces are observed for both linear and Hertzian contacts, even though less so for the latter, irrespective of compression level and even for extremely high tangential contact stiffness. We compare the data from DEM simulations with predictions from well-established micromechanical models, namely the Effective Medium Theory (EMT) and the Fluctuation Theory (FT). Both theories do not account for the effect of different preparation history (different inter-particle friction coefficients) on the elastic moduli. The fluctuation theory is in agreement with numerical data with Hertzian particles, almost perfect for the bulk modulus and close for the shear modulus, at least in the intermediate compression regime, but does not capture the anomalous behavior (small and fairly constant of the tangential shear modulus) where the theory overpredicts. When looking at the fluctuations in the normal displacements, we find good quantitative and qualitative agreement with the functional form assumed by the theory for Hertzian contacts, whereas the dependence on the tangential stiffness leads to major deviations in the case of linear contacts. 1

\subsection{Introduction}

Granular materials behave differently from usual solids or fluids and show peculiar mechanical properties like dilatancy or ratcheting. One interesting characteristics of these materials is their sensitivity to external stress. In densely packed systems, for instance, the external pressure can cause the system to transition between rigid and floppy states, the so-called jamming transition, typical of many amorphous materials. Since the system becomes rigid and can carry loads, jamming defines the threshold at which the elastic stiffness of the assembly assumes finite values $[37,122,148]$. The solid states above jamming are the focus of this study.

The response of granular solids to small strains is relevant for many industrial and geotechnical applications. In these cases, where strains are small, a

\footnotetext{
${ }^{1}$ Taghizadeh, K., Luding, S., \& Magnanimo, V. International Journal of Solids and Structures, submitted Aug. 2018.
} 
sound knowledge of the bulk stiffness is essential for the realistic prediction of the macroscopic behaviour $[35,233]$ which can be highly non-linear due to disorder and involves irreversibility (plasticity), due to frictional rearrangements of the elementary particles $[8,16,70,221]$. In micromechanical-based models, this feature is often neglected and elastic properties are associated with the deformations in a fixed contact network, and therefore correspond to the "true elastic" stiffness [28, 74, 159].

From the theoretical point of view a popular approach assumes a uniform strain at all scales, i.e. the displacement field of the grains is affine with the macroscopic deformation, and only the stiffness tensor is needed to compute the stresses in terms of strains. This approach is usually referred to as the Effective Medium Theory (EMT) [94, 159]. EMT successfully describes the behavior of ordered granular systems, given the microstructural unit arrangement and the external condition (pressure and volume fraction) [165, 172]. However, in the case of disordered media, displacements of particles in contact do not follow the affine motion and the corresponding prediction significantly overestimates the actual moduli, especially for loose systems. By assuming an affine motion for all particles, one can only obtain an upper bound for the effective elastic moduli [39, 47, 180, 253]. Efforts have been made to improve upon EMT. Introducing fluctuations of particles displacements is an important first step to consider non-affinity [93, 168, 248]. In Ref. [93], an analytical expression of the elastic moduli was derived for an assembly of identical frictionless particles by applying the pair fluctuation model. Later, the given expression was extended to frictional particle contacts considering the interaction of particle pairs with their neighbors [125].

Various authors have compared the predicted values of the effective bulk and shear moduli of granular assemblies with the results of DEM simulations $[2,105,116,159,229]$ and physical experiments [94, 159, 160, 236]. It has been shown that a proper description of the microstructure, as related to both particle characteristics and load history, is essential for the characterization of granular elasticity [155, 233].

In this study, we use Discrete Element simulations to prepare and test frictional, disordered granular samples in isotropic conditions and to study and understand their effective (bulk) elastic behavior. We scan a wide range of inter-particle friction coefficients, contact stiffnesses and confining pressures, in order to understand how the interplay of contact and system properties affects the elastic moduli. Two popular normal contact models are implemented, namely linear visco-elastic and non-linear Hertzian, and their influence on the tangential effective properties is carefully assessed. Furthermore, we compare 
analytical expressions with results from simulations and address the importance of displacement fluctuations, measured directly from DEM results.

This paper is organized in the following way. We first recall the properties of the model material and we define some micro- and macro-mechanical quantities in Sec. 3.2. Next, in Sec. 3.3, we describe the sample preparation protocol, and explain the approach to calculate the effective elastic moduli. After that, results obtained for samples prepared with different friction coefficients in case of linear and Hertzian contact models are discussed. In Sec. 3.4, we briefly summarize the theoretical frameworks of interest, and their predictions for the elastic moduli and compare them with DEM results. Sec. 3.5 focuses on the role of tangential stiffness on the elastic properties and the interplay with contact fluctuations. Finally, we summarize our observations in Sec. 3.6 with conclusions and a final outlook.

\subsection{Numerical setup}

The Discrete Element Method (DEM) [41, 150] can help to understand and predict the stress response to deformations of particle systems. At the basis of DEM are laws that relate the interaction force to the overlap and tangential displacement of two particles in contact. If all forces $\mathbf{f}_{i}$ acting on particle $i$ are known, the problem is reduced to the integration of Newton's equations of motion for the translational and rotational degrees of freedom. The system contains 4096 polydisperse frictional spheres in a triaxial periodic box. Gravity is neglected in all simulations, so that during preparation, after the applied isortopic compression, the system still is nearly isotropic and homogeneous.

\subsubsection{Contact models}

The normal contact force model is given by $f^{n}=k_{n} \delta^{n}+\gamma_{n} \dot{\delta}^{n}$, where $k_{n}$ is the normal spring stiffness, $\gamma_{n}$ is the normal contact viscosity parameter, $\delta^{n}=$ $\left(d_{i}+d_{j}\right) / 2-\left(\mathbf{r}_{i}-\mathbf{r}_{j}\right) . \hat{\mathbf{n}}$ is the overlap in normal direction between two interacting particles $i$ and $j$ at positions $\mathbf{r}_{i}$ and $\mathbf{r}_{j}$, with diameters $d_{i}$ and $d_{j}$, with contact normal vector $\hat{\mathbf{n}}=\left(\mathbf{r}_{i}-\mathbf{r}_{j}\right) /\left|\left(\mathbf{r}_{i}-\mathbf{r}_{j}\right)\right|$, and $\dot{\delta}^{n}$ is the relative velocity in the normal direction.

In this work, two contact models are compared, namely Hertzian viscoelastic and linear visco-elastic contact models for the normal force. In case of the Hertzian contact model, the normal spring and contact viscosity are functions of particle overlap and expressed as $k_{n}^{H}=\frac{G_{p}}{1-v} \sqrt{\bar{d} \delta^{n}}$ and $\gamma_{n}^{H}=\alpha_{n} \sqrt{\bar{d} \delta^{n}}$, 
respectively, with the shear modulus of particles $G_{p}$, the Poisson's ratio of particles $v$, and a Hertzian viscous dissipation parameter $\alpha_{n}$. For the linear contact model, the stiffness $\left(k_{n}^{L}\right)$ and viscosity $\left(\gamma_{n}^{L}\right)$ remain constant, independent of particles overlap. In order to reduce dynamical effects and shorten relaxation times, an artificial viscous background dissipation force and torque, $\mathbf{f}_{i}^{b}=-\gamma_{t r} \mathbf{v}_{i}$ and $\mathbf{t}_{i}^{b}=-\gamma_{\text {rot }}$, proportional to the moving velocity $\mathbf{v}_{i}$ of particle $i$ is added, resembling the damping due to a background medium, as e.g. a fluid, but not affecting the elastic properties.

Friction is generated by the relative motion of the two particles in contact, and is modeled according to the Coulomb law. The tangential force $f^{t}$ exerted between two particles is given by $f^{t}=k_{t} \delta^{t}+\gamma_{t} \dot{\delta}^{t}$, where $k_{t}$ is the tangential spring stiffness, $\gamma_{t}$ is the tangential contact viscosity parameter, $\delta^{t}$ is the displacement and $\dot{\delta}^{t}$ is the relative velocity in the tangential direction [150]. In case of the Hertzian contact model, the tangential spring and contact viscosity are functions of the normal overlap $\delta^{n}$ as $k_{t}^{H}=\frac{2 G_{p}}{2-v} \sqrt{\bar{d} \delta^{n}}$ and $\gamma_{t}^{H}=\alpha_{t} \sqrt{\bar{d} \delta^{n}}$. In the case of linear contact model, the tangential stiffness and viscosity, $k_{t}^{L}$ and $\gamma_{t}^{L}$, remain constant. When the tangential force exceeds the maximum allowed value of the force $f^{t}=\mu f^{n}$, the two particles slide. Fig. 3.1.a and b illustrate the normal and tangential contact models described in this section.

\subsubsection{Simulation parameters}

The standard simulation parameters are $N=4096\left(=16^{3}\right)$ particles with average diameter $\bar{d}[\mathrm{~mm}]$, and material density $\beta=2000\left[\mathrm{~kg} / \mathrm{m}^{3}\right]$, while the coefficient of friction $\mu$ assumes values between 0 and 1 . To avoid crystallization, polydispersity is introduced, with $w=d_{\max } / d_{\min }=3$, the width of a uniform size distribution, where $d_{\max }$ and $d_{\min }$ are the diameters of the biggest and smallest particle respectively. Numerical values of all the parameters used in DEM simulations are presented in Table 3.1.

The typical time scale for the interaction of two particles with masses $m_{i}=\beta\left(\pi d_{i}^{3} / 6\right)$ and $m_{j}=\beta\left(\pi d_{j}^{3} / 6\right)$ is $t_{c}=\pi / \sqrt{k_{n} / m_{i j}-\left(\gamma_{n} / 2 m_{i j}\right)^{2}}$, where $m_{i j}=$ $m_{i} m_{j} /\left(m_{i}+m_{j}\right)$ is the reduced mass. The restitution coefficient quantifies the dissipation for a collision of a pair of particles, $e=\exp \left(-\gamma_{n} t_{c} / 2 m_{i j}\right)$. The size of particles is thus a parameter that affects the contact duration $t_{c}$ and restitution coefficient $e$, when particles have the same stiffness and viscosity. Since our distribution is polydisperse, the fastest possible collision takes place between two smallest particles in the overall ensemble with $t_{c}=0.228[\mu s]$ and $e=0.804$ (and $t_{c}=0.643[\mu s]$ and $e=0.926$ for the mean size particles) [91]. In case of Hertzian 


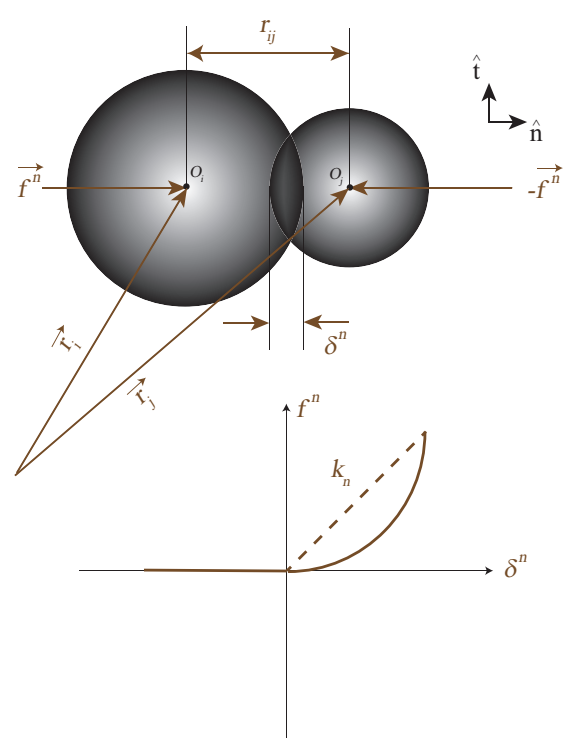

(a)

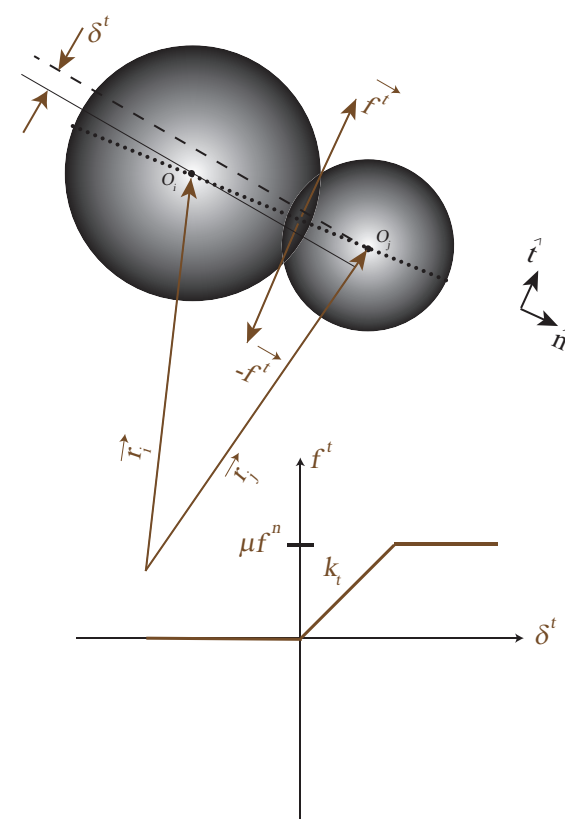

(b)

Figure 3.1: (a) Normal contact models with the normal overlap distance $\delta^{n}$. (b) Tangential contact models with the tangential distance $\delta^{t}$. The tangential force is coupled to the normal force via Coulomb's law.

contacts, $t_{c}$ varies with the maximal overlap between particles. However, in this study, we consider a constant minimal $t_{c}$, calculated based on the interaction time-scale of two particles with diameter $d_{\min }$ at the maximum expected pressure level and thus overlap ${ }^{2}$. Consequently, the integration time-step is set to $\Delta t=0.004[\mu s]$, approximately 50 times smaller than the smallest collision time $t_{c}$, in the two cases of linear and Hertzian contacts.

\footnotetext{
${ }^{2}$ One can find a linearized stiffness of Hertzian from a force-displacement plot of a two particle collision. In this work, the Hertzian stiffness was linearized at the maximum overlap to estimate the worst case time step as $\Delta t \approx 0.004[\mu \mathrm{s}]$.
} 


\begin{tabular}{llll}
\hline Parameter & Value & S.I. Units & Description \\
\hline$t_{u}$ & 1 & $1[\mu \mathrm{s}]$ & Time unit \\
$l_{u}$ & 1 & $1[\mathrm{~mm}]$ & Length unit \\
$m_{u}$ & 1 & $1[\mu \mathrm{g}]$ & Mass unit \\
$N$ & 4096 & 4096 & Number of particles \\
$\bar{d}$ & 2 & $2[\mathrm{~mm}]$ & Average diameter \\
$w$ & 3 & $3[-]$ & Polydispersity $w=\frac{d_{\max }}{d_{\min }}$ \\
$\beta$ & 2000 & $2000\left[\mathrm{~kg} / \mathrm{m}^{3}\right]$ & Density \\
$\mu$ & varied $[0-1]$ & $0-1[-]$ & Friction coefficient \\
$G_{p}$ & $29 \cdot 10^{3}$ & $29 \cdot 10^{9}\left[\mathrm{~kg} / \mathrm{m} . \mathrm{s}^{2}\right]$ & Shear modulus of grains \\
$v$ & 0.2 & $0.2[-]$ & Poisson's ratio of grains \\
$k_{n}^{L}$ & $10^{5}$ & $10^{8}\left[\mathrm{~kg} / \mathrm{s}^{2}\right]$ & Stiffness-normal spring \\
$k_{t}^{L}$ & 80000 & $8 \cdot 10^{7}\left[\mathrm{~kg} / \mathrm{s}^{2}\right]$ & Stiffness-tangential spring \\
$\gamma_{n}^{L}$ & 1000 & $1[\mathrm{~kg} / \mathrm{s}]$ & Linear viscous normal dissipation \\
$\gamma_{t}^{L}$ & 200 & $0.2[\mathrm{~kg} / \mathrm{s}]$ & Linear viscous tangential dissipation \\
$\gamma_{t r}$ & 100 & $0.1[\mathrm{~kg} / \mathrm{s}]$ & Background damping-translation \\
$\gamma_{r o t}$ & 20 & $0.02[\mathrm{~kg} / \mathrm{s}]$ & Background damping-rotational \\
$\alpha_{n}$ & 1000 & $1[\mathrm{~kg} / \mathrm{m} . \mathrm{s}]$ & Hertzian viscous normal dissipation \\
$\alpha_{t}$ & 20 & $0.2[\mathrm{~kg} / \mathrm{m} . \mathrm{s}]$ & Hertzian viscous tangential dissipation \\
\hline
\end{tabular}

Table 3.1: Numerical values of particle parameters used in the DEM simulations

\subsubsection{Characteristic quantities}

Here, we present the general definitions of averaged microscopic and macroscopic quantities relevant for analyses in the following sections. Microquantities are often impossible to measure in experiments but are easily available from DEM simulations. The classical definition of the coordination number is $M / N$, where $M$ is the total number of contacts and $N$ is the total number of particles. Because there is no gravity in our simulations, at the end of relaxation there will usually be particles without contacts, or due to numerical precision, particles with fewer contacts than needed to keep them in equilibrium. As we are interested to link the macroscopic load carried by the sample with the microscopic contact network, all particles with less than 3 contacts (so-called rattlers) are excluded, since they are not mechanically stable and do not contribute to the force network. Excluding rattlers leads to the corrected coordination number $\bar{Z}=M_{3} / N_{3}$, where $M_{3}$ is the total number of contacts of the $N_{3}$ particles with at least 3 contacts. The volume fraction $\phi$ is given by the ratio between the volume of the particles and the total volume, $\phi=\sum_{p=1}^{N} \frac{V_{p}}{V}=\frac{N}{V} \frac{\pi}{6} \bar{d}^{3}$ ignoring the overlaps. 
Furthermore, we can define averaged tensorial macroscopic quantities including strain- and stress-tensor. The strain tensor $\varepsilon_{i j}$ is the external (global) strain that we apply to the sample. The isotropic part of the infinitesimal strain is defined as $\varepsilon_{v o l}=\dot{\varepsilon}_{v o l} \Delta t=-\left(\varepsilon_{x x}+\varepsilon_{y y}+\varepsilon_{z z}\right) / 3=\left(-\varepsilon_{\alpha \alpha}\right) / 3=\left(-\dot{\varepsilon}_{\alpha \alpha} \Delta t\right) / 3$, where $\varepsilon_{\alpha \alpha}=\dot{\varepsilon}_{\alpha \alpha} \Delta t$ with $\alpha \alpha=x x, y y$ and $z z$ as the diagonal components of the tensor in the Cartesian $x-y-z$ reference system and $\dot{\varepsilon}_{v o l}$ is the strain-rate. The trace integral of $3 \varepsilon_{v o l}$ denoted by $\varepsilon_{v}$ is the true logarithmic strain, i.e. the volume change of the system relative to a reference volume, $V_{0}$, or the true isotropic strain.

From DEM simulations, one can measure the "static" stress in the system:

$$
\sigma_{i j}=(1 / V) \sum_{c \in V} l_{i}^{c} \otimes f_{j}^{c},
$$

averaged over all contacts in the volume $V$, with the dyadic product between the branch vector $l_{i}^{c}$ and the contact force $f_{j}^{c}[33,72,91,92]$. As here branch vector and force are defined to be parallel, the isotropic component of the stress is the pressure $P=\sigma_{\alpha \alpha} / 3$.

Note that in this work we use $k^{*}=k_{n} / \bar{d}\left(k_{n}^{H}\right.$ and $k_{n}^{L}$ for Hertzian and linear contact models, respectively) to non-dimensionalize macro-quantities, e.g., $\sigma_{i j}^{*}=\sigma_{i j} / k^{*}$ and $P^{*}=P / k^{*}$,

\subsection{DEM simulations}

\subsubsection{Preparation procedure}

The preparation procedure is an essential step in any physical/numerical experiment to obtain reproducible and reliable results, especially when friction is involved. In this work, the preparation procedure starts with spherical particles randomly generated, with low volume fraction $\phi_{g}$ and rather large random velocities, in a periodic 3D box, such that they have sufficient space and time to randomize themselves. The initial configurations are obtained by first homogeneously compressing this granular gas up to a volume fraction $\phi_{\mathrm{o}}$ below the transition from solid-like to liquid-like behavior, the so-called jamming transition $\phi_{j}$ $[122,148]$. The system is then relaxed to allow the particles to dissipate kinetic energy and achieve zero-pressure. This is followed by an isotropic compressiondecompression cycle up to a desired maximum volume fraction $\phi_{\max }=0.82$, as depicted in Fig. 3.2 [73, 91, 233]. Different coefficients of friction, from $\mu=0$ to 
1 , are used during the preparation process. To prevent inhomogeneity, the simulations are carried out by applying a uniform strain field to the system where at every time-step $\Delta t$ the particles move according to the momentary strain-rate tensor $\dot{\varepsilon}_{i j}= \pm \dot{\varepsilon}_{v o l} \delta_{i j}$ with unit tensor $\delta_{i j}$ and rate $\dot{\varepsilon}_{v o l}=12 \cdot 10^{-6}$; positive and negative sign mean compression and decompression, respectively.

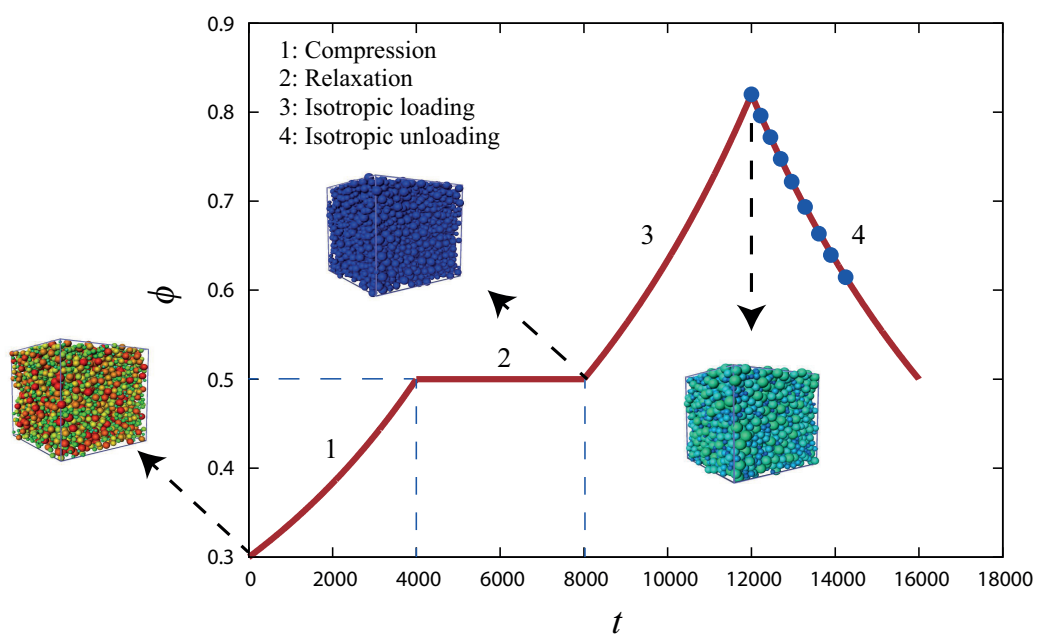

Figure 3.2: Evolution of volume fraction as a function of time during sample preparation: (1) A frictional granular gas is homogeneously compressed from $\phi_{g}=0.3$ to $\phi_{\circ}=0.5$; and (2) relaxed at $\phi_{\circ}=0.5$; (3) the sample is compressed from $\phi_{\circ}=0.5$ to $\phi_{\max }=0.82$; (4) finally, the sample is decompressed from $\phi_{\max }=$ 0.82 to $\phi_{\circ}=0.5$. Blue bullets ' $\bullet$ represent configurations chosen for further tests. The color of particles indicates their average overlap. Large (artificial) overlaps are present in the initial random gas (red particles), whereas in the relaxed packing (blue) particles practically do not overlap.

\subsubsection{Elastic stiffness}

We now discuss the conditions for which the response to load increments of a pre-stressed granular packing in mechanical equilibrium can be described as elastic, and explain how macroscopic elastic moduli are computed in our simulations.

After sample preparation with different contact friction, different system 
configurations are realized. We study their incremental response during isotropic compression and plane pure shear tests. Various configurations are chosen at different volume fractions above jamming, along the unloading branch (blue dots in Fig. 3.2). Then, sufficient relaxation is applied to allow the particles to dissipate their kinetic energy and achieve a static configuration in mechanical equilibrium. After that, we probe these relaxed samples by applying small strain perturbations and measure the incremental stress responses $[62,91,121,126,155]$.

For each configuration, we apply identical strains $\varepsilon_{v}=3 \varepsilon_{v o l}=\varepsilon_{x x}+\varepsilon_{y y}+$ $\varepsilon_{z z}=3 \cdot 10^{-6}$ and $\varepsilon_{d e v}=\varepsilon_{x x}-\varepsilon_{y y}=2 \cdot 10^{-6}$. For each calculation, we verify that the applied strain is small enough to be in the linear, elastic, reversible regime where the coordination number does not vary significantly. A general schematic representation of the sample isotropic and deviatoric tests is shown in Fig. 3.3.

In this stage, the friction coefficient is set to infinity $(\mu=\infty$, i.e. a large value) to avoid sliding of contacts during probing. In appendix. A, we discuss the case where we keep the friction between particles during the probing the same as during sample preparation, as well as the importance of preventing slippage.

After probing the configurations, the effective elastic moduli of the isotropic granular assembly are obtained as the ratio between the measured increment in stress and the applied strain:

$$
\begin{aligned}
& K^{*}=\left.\frac{\Delta P^{*}}{\Delta \varepsilon_{v}}\right|_{\Delta \varepsilon_{d e v}=0} \\
& G^{*}=G_{x y}^{*}=\left.\frac{\Delta \sigma_{d e v}^{*}}{2 \Delta \varepsilon_{d e v}}\right|_{\Delta \varepsilon_{v}=0}=\left.\frac{\Delta\left(\sigma_{x x}^{*}-\sigma_{y y}^{*}\right)}{2 \Delta\left(\varepsilon_{x x}-\varepsilon_{y y}\right)}\right|_{\Delta \varepsilon_{v}=0}
\end{aligned}
$$

while $K^{*}=K \bar{d} / \bar{k}_{n}$ and $G^{*}=G \bar{d} / \bar{k}_{n}$ are the non-dimensional moduli. The stiffness $\bar{k}_{n}$ assumes different expressions in the two cases of linear and Hertzian contact models. In the former $\bar{k}_{n}=k_{n}^{L}$ is constant and the value is given in Table. 3.1, in the latter $\bar{k}_{n}=k_{n}^{H}$ depends on the sample configuration via the average overlap $\bar{\delta}^{n}$, as $\bar{k}_{n}^{H}=\frac{G_{p}}{1-v} \sqrt{\bar{d} \bar{\delta}^{n}}$. As samples are isotropic, the shear moduli along different directions are assumed to be identical $G^{*} \simeq G_{x y}^{*} \simeq G_{y z}^{*} \simeq G_{z x}^{*}$, as tested and confirmed in a few cases (data not shown). 


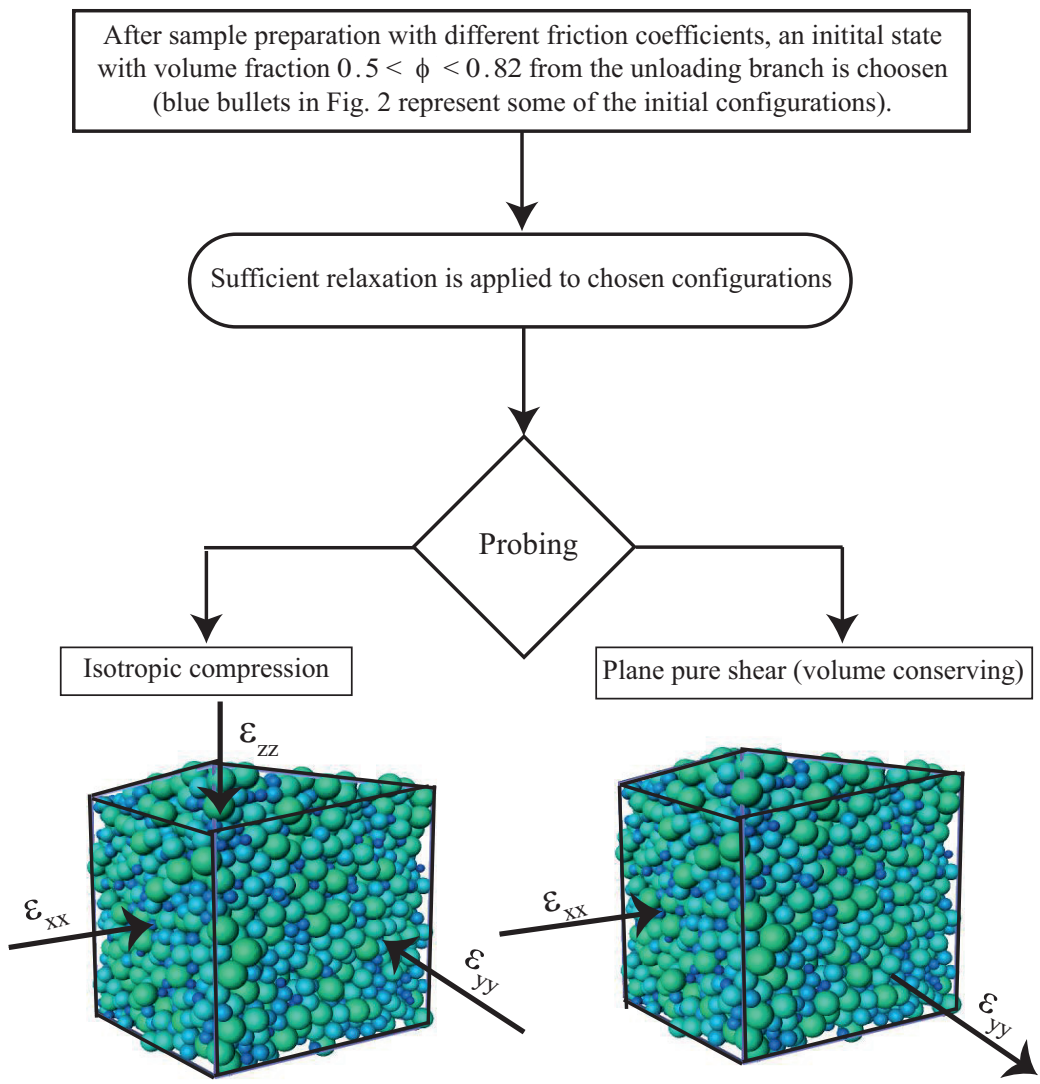

Figure 3.3: Schematic representation of the procedure for implementing isotropic and deviatoric deformation probing in the cubic triaxial element test.

\subsubsection{Influence of inter-particle contact friction during preparation on the elastic moduli}

Using the probing approach explained in Sec. 3.3.2, we next study the incremental elastic response of the aggregates and its dependence on the actual configuration and deformation history. In Fig. 3.4, we plot the variation of the normalized moduli $K^{*}$ and $G^{*}$ against the product of volume fraction and coordination number $\phi \bar{Z}$, for packings prepared with different coefficients of 
friction, $\mu$, in the cases of linear and Hertzian contacts. For both models, as expected, the elastic moduli increase with increasing $\phi \bar{Z}$. However, the increase of the shear modulus is slower for packings prepared with higher friction. We can relate this behavior to the lower average number of contacts for samples prepared with higher friction at the same volume fraction [74]. For smaller friction coefficients, the tangential components of forces are typically reduced as are the rotational degrees of freedom, which leads to static packings with more contacts. It is worth pointing out that the coefficient of friction has no direct influence on the elastic moduli during probing, as sliding is forbidden $(\mu=\infty)$, for details see appendix. A. Rather $\mu$ affects $K^{*}$ and $G^{*}$ indirectly through the sample preparation that leads to different $\bar{Z}$, i.e., to different micro-structure. Both the contact model and the preparation procedure have a crucial influence on the transition regime, where increasing friction coefficients shift the jamming transition to the left, to smaller $\phi$ and $\bar{Z}$, for both linear and Hertzian cases. For all cases, the effect becomes negligible and the behaviour seems to saturate for friction coefficients $\mu \geq 0.5$.

The figure also gives an overview about the differences in the compressional/shear elastic moduli associated with the contact model. For both contact models, the bulk modulus $K^{*}$ (Fig. 3.4a and c) linearly scales with $\phi \bar{Z}$ over a wide range of values, with deviation and discontinuity only for very loose samples (low $\phi \bar{Z}$ ). The Hertzian data collapse whereas the linear data slightly decrease with $\mu$ even though both had the same preparation history. . However, values of the normalized modulus $K^{*}$ are slightly higher in the case of Hertzian interactions than for linear ones. As for $G^{*}$, the linear contact model seems underestimate the values of the Hertzian shear stiffness, especially for large $\phi \bar{Z}$ and high friction. The preparation procedure affects the shear modulus in the whole $\phi \bar{Z}$ regime and a linear scaling $G^{*} \sim \phi \bar{Z}$ is not retrieved for neither linear nor for Hertzian packings $[4,105,182]$. The dependence of $G^{*}$ on the history appears more dramatic for linear contacts than for Hertzian. In fact, data in the intermediate regime $(3 \leq \phi \bar{Z} \leq 4.5)$ overlap in Fig. 3.4(d), the same does not happen in Fig. 3.4(b). On the other hand, for highly deformed packings ( $\phi \bar{Z} \geq 4.5$ ), $G^{*}$ tends to constant values. Again, this trend is more pronounced for linear contacts than for Hertzian.

\subsection{Granular elasticity}

The simplest approach to model elasticity in granular materials is given by the Effective Medium Theory (EMT), as proposed initially by Walton [253] and 


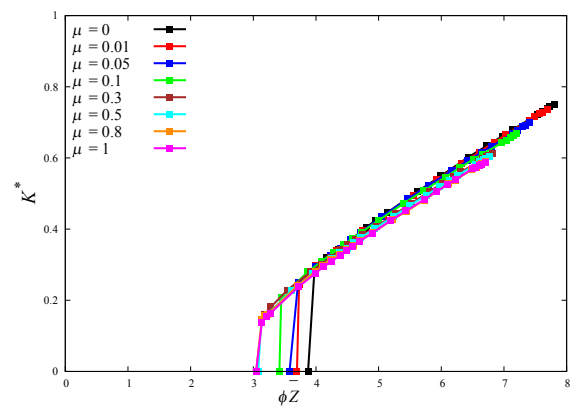

(a)

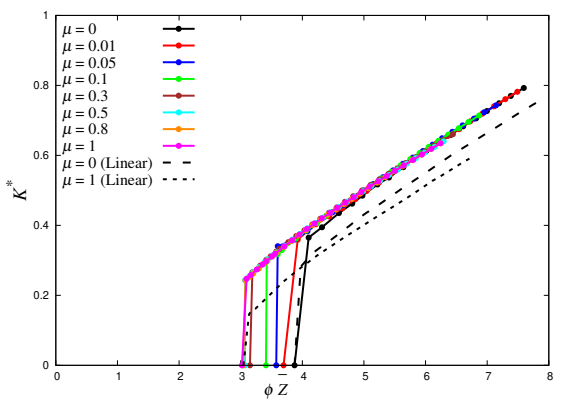

(c)

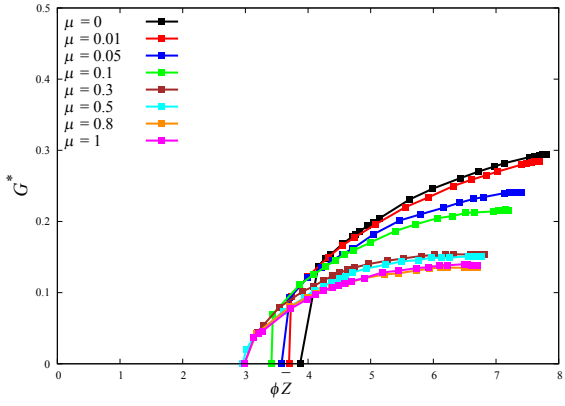

(b)

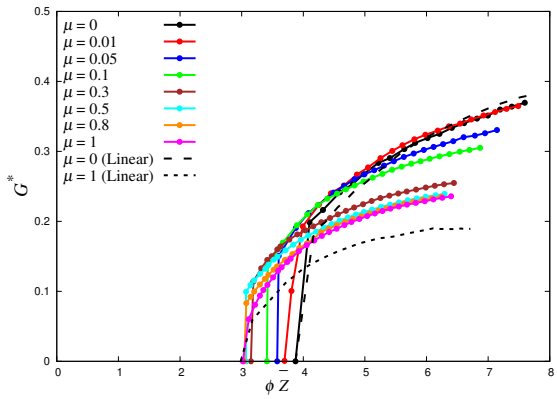

(d)

Figure 3.4: Evolution of the normalized bulk modulus $K^{*}$ and shear modulus $G^{*}$ with the product of volume fraction and coordination number $\phi \bar{Z}$ for different coefficients of friction, $\mu$, during preparation as shown in the legend, and $\mu=$ $\infty$ during probing, for packings that interact through linear (a) and (b) and nonlinear (c) and (d) normal contacts. Dashed and dotted lines in subfigures (c) and (d) refer to the values of $K^{*}$ and $G^{*}$ obtained with a linear contact model as in subfigures (a) and (b) and low/high values of friction ( $\mu=0$ and $1)$.

Digby [47]. EMT assumes that all particles move according to the affine motion defined by the applied, external strain rate field $\dot{\varepsilon}_{i j}$. That is the relative incremental displacement rate between a pair of contacting particles $A B$ would be:

$$
\dot{u}_{i}^{(B A)}=\dot{\varepsilon}_{i j} l_{j}^{(B A)},
$$


where $\dot{u}_{i}^{(B A)}=\dot{u}_{i}^{(B A)}=\dot{u}_{i}^{(B)}-\dot{u}_{i}^{(A)}$ and $l_{i}^{(B A)}$ is the branch vector introduced in Sec. 3.2.3.

\subsubsection{Effective medium theory}

Based on the affine assumption for particle displacement, the following expressions for the bulk and shear moduli of an isotropic aggregate of polydisperse particles are obtained:

$$
\begin{aligned}
K^{*} & =\frac{\phi \bar{Z}}{3 \pi} D \\
G^{*} & =\left(1+\frac{3}{2} \frac{\bar{k}_{t}}{\bar{k}_{n}}\right) \frac{\phi \bar{Z}}{5 \pi} D,
\end{aligned}
$$

with $\bar{k}_{n}$ and $\bar{k}_{t}$ average normal and tangential stiffness, and $\bar{d}^{2}$ and $\bar{d}^{3}$ second and third moment of the size distribution, respectively. The term $D=\frac{\bar{d} \bar{d}^{2}}{\bar{d}^{3}}$ is based on the actual sample configuration and corrects the expressions of the moduli to take into account polydispersity [29, 115, 215]. In this work $D=\frac{\bar{d} \bar{d}^{2}}{\bar{d}^{3}} \approx 1.15$. Eqs. (3.4) give the EMT predictions of elastic moduli of disordered systems of frictional particles in 3D as functions of volume fraction $\phi$ and coordination number $\bar{Z}$. The linear contact stiffnesses $k_{n}^{L}$ and $k_{t}^{L}$ are constant, and the moduli do not explicitly depend on pressure $P$. Differently, in the case of Hertzian interactions, an extra dependence on the average overlap, and in turn pressure, appears in Eqs. (3.4), via the contact stiffness [125, 159], as defined in Sec. 3.2 with the average overlap between particles [125]:

$$
\frac{\bar{\delta}_{n}}{\bar{d}} \cong\left[\frac{3 \pi}{2} \frac{(1-v)}{\phi \bar{Z}} \frac{P}{G_{p}}\right]^{2 / 3} .
$$

The expressions in Eqs. (3.4) have been widely used for a number of applications, see among others [18, 94, 118, 159, 168, 215]. While EMT describes exactly the response of regular arrays of particles $[165,172]$, the affine assumption overpredicts $[155,159]$ the elastic moduli of a random granular assembly, especially in the case of loose samples subjected to shear probing.

\subsubsection{Fluctuation theory}

In order to improve the theoretical prediction, particle displacements can be given by the sum of the affine average and additional fluctuation components 
[28, 93, 111, 116, 125]. Then, Eq. (3.3) becomes [93, 125]:

$$
\dot{u}_{i}^{(B A)}=\dot{\varepsilon}_{i j} l_{j}^{(B A)}+\dot{\Delta}_{i}^{(B A)}-\frac{1}{2} \epsilon_{i j k} \dot{S}_{j}^{(B A)} l_{k}^{(B A)},
$$

where the first term is the affine displacement rate, and $\dot{\Delta}_{i}^{(B A)}$ is the fluctuation in the translations of the two centres, while $\dot{S}_{j}^{(B A)}$ is the fluctuations due to the rotations about their centres, and $\epsilon_{i j k}$ is the permutation symbol. The so-called fluctuation theory (FT) provides an analytical formulation for the bulk and the shear moduli of a random granular aggregate $[93,125]$ :

$K^{*}=\left[1-2\left(\rho+\rho^{*}\right)\right] \frac{\phi \bar{Z}}{3 \pi} D$

$G^{*}=\left[1-2\left(\rho+\rho^{*}\right)\right] \frac{\phi \bar{Z}}{5 \pi} D+\frac{3}{2} \frac{\bar{k}_{t}}{\bar{k}_{n}}\left[1-2\left(\rho+\rho^{*}\right)+2\left(\zeta+\zeta^{*}\right)\right] \frac{\phi \bar{Z}}{5 \pi} D$,

where the coefficients $\rho$ and $\zeta$ are averages of the incremental fluctuations in displacement with $\rho^{*}=\rho(1-2 \rho+2 \zeta)$ and $\zeta^{*}=\zeta(1-2 \rho+2 \zeta)$ (the steps to obtain Eqs. (3.7) are briefly given in appendix. B, see [125] for details). Fluctuations relax the relative displacement between particle pairs, and the coefficients $\rho$ and $\zeta$ introduce a reduction term that lowers the values of the moduli predicted by EMT. In particular, in Ref. [125] the authors derived an explicit expression for $\rho$ and $\zeta$. They are given as a statistical measure of the sample contact geometry and involve only the particle coordination number:

$\rho=\frac{22-3 \bar{Z}}{24}\left(\frac{1}{\bar{Z}}-\frac{1}{\bar{Z}^{2}}\right)+\frac{1}{8} \chi$

$\zeta=-\frac{66-9 \bar{Z}}{26 \bar{Z}}\left(1-\frac{24}{13 \bar{Z}}\right)-\frac{261}{676} \chi$,

with standard deviation $\chi=\overline{(Z-\bar{Z})^{2}} / \bar{Z}^{2}$ of the contact number per particle, $Z$.

Next, we measure the contact fluctuations in our DEM packings and we explore the relation between $\chi$ and the microstructure parameter $\phi \bar{Z}$ in Fig. 3.5. Interestingly, we observe that the data collapse irrespective of the contact model and preparation procedure. We can then extract an approximate analytical expression for $\chi$ as a function of volume fraction and coordination number as:

$\chi=(\phi \bar{Z})^{-1}$.

Using Eq. (3.10) in Eqs. (3.8) and (3.9) and we plot the predictions of FT and EMT for $K^{*}$ and $G^{*}$ in Fig. 3.6, versus $\phi \bar{Z}$, along with linear and Hertzian 


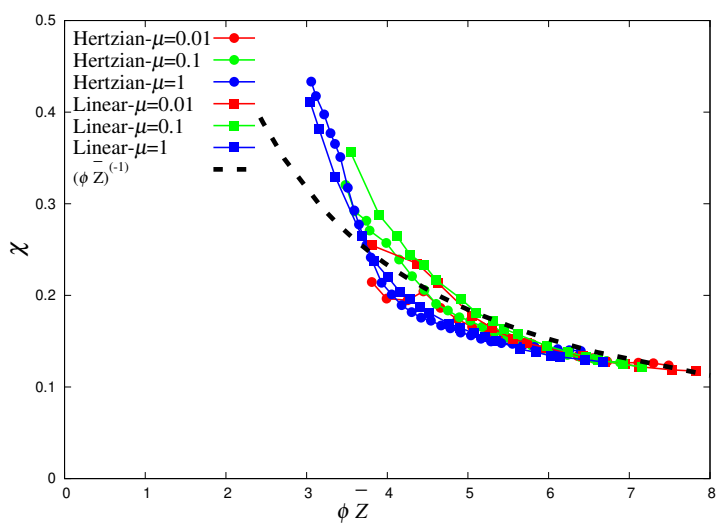

Figure 3.5: Change of $\chi$ with respect to the product of volume fraction and coordination number $\phi \bar{Z}$ for different coefficients of friction, $\mu$, as shown in the legend. The dotted black line represents the fitting expression in Eq. (3.10).

numerical data. Data from [155] are included as well. Since, the normalized moduli $K^{*}=\frac{K \bar{d}}{\bar{k}_{n}}$ and $G^{*}=\frac{G \bar{d}}{\bar{k}_{n}}$ are used, the predictions of EMT and FT for linear and Hertzian contact packings coincide.

As reported earlier $[116,160]$, simulation data are in reasonable agreement with EMT for the bulk modulus $K^{*}$, irrespective of the contact model details and the preparation procedure. The predictions for $K^{*}$ become excellent when FT and Hertzian simulations are compared. Yet, the theory can not capture the behavior for very loose samples, $\phi \approx \phi_{j}$.

On the other hand, the shear modulus does not scale with the product of the state variables as proposed by Eqs. (3.4) and (3.7). A good agreement between fluctuation theory and samples with Hertzian contacts is found in the intermediate $\phi \bar{Z}$ regime. For highly compressed samples $(\phi \bar{Z}>4.5$, data shown with dotted lines), $G^{*}$ deviates considerably from the analytical prediction. The deviation is partly due to the high overlap between particles at such a compressed state, where the binary contact assumption of DEM is not valid anymore. Moreover, the theory overpredicts $G^{*}$ for small $\phi \bar{Z}$ where the jamming transition strongly depends on the preparation history. The mismatch between theory and simulations is worse in the case of linear contacts. 


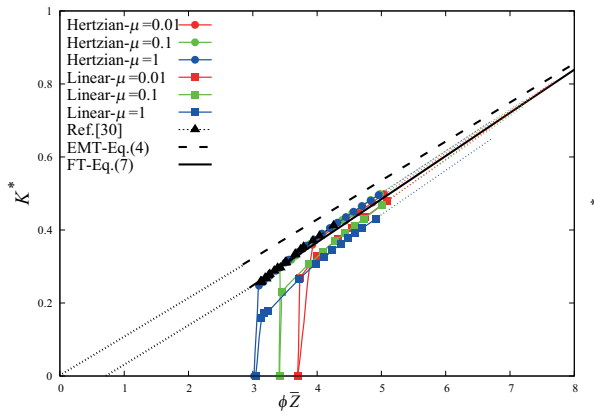

(a)

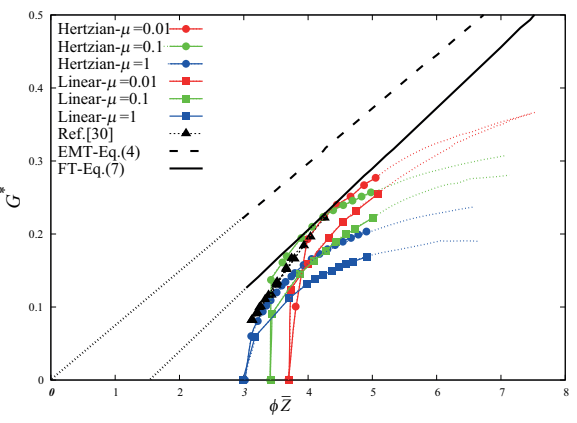

(b)

Figure 3.6: Evolution of the normalized (a) bulk modulus $K^{*}$ and (b) shear modulus $G^{*}$ with the product of volume fraction and coordination number $\phi \bar{Z}$ for different contact models and coefficients of friction, $\mu$, as shown in the legend. Dashed and solid lines are Eq. (3.4) and Eq. (3.7), respectively. Thin lines extend the simulations to very high overlaps, where the contact models reach their limits.

\subsection{Role of the tangential stiffness during probing}

After studying the effect of contact friction during preparation, and probing with $\mu=\infty$ and fixed $k_{t}$, in this section, we investigate the influence of the tangential stiffness on the elastic response of the material. We start from the samples prepared with linear contacts and frictionless $\left(\mu=0\right.$ and $\left.k_{t}=0\right)$ and probe the elastic response by assigning a finite value to the tangential stiffness in proportion to the constant value of $k_{n}^{L}$ (Table 3.1), and a very high value for the friction coefficient. Two extreme exemplary values of $k_{t}^{L} / k_{n}^{L}$ are considered, namely $k_{t}^{L} / k_{n}^{L}=0.1$ and 0.8 . With this approach, at a given density, the packings with different tangential stiffness have exactly the same particle/contact network and rearrangements are prevented during small strain probing.

Along with the linear cases, Hertzian packings are studied, characterized by the ratio $k_{t}^{H} / k_{n}^{H}=0.88$, and the other material constants given in Table 3.1. In this case, a friction coefficient $\mu=0$ is used during preparation and $\mu=\infty$ during probing, similar to the linear packings.

Fig. 3.7 shows results for the bulk and shear moduli versus the product of volume fraction and coordination number $\phi \bar{Z}$. Despite packings at a given $\phi \bar{Z}$ being topologically identical, the activation of the tangential stiffness during 


\section{Micromechanical study of the elastic stiffness in isotropic frictional granular solids}

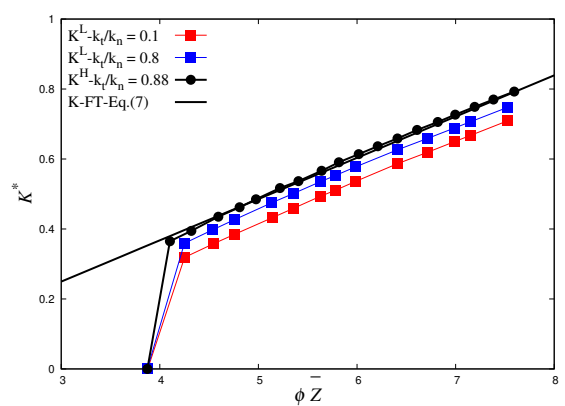

(a)

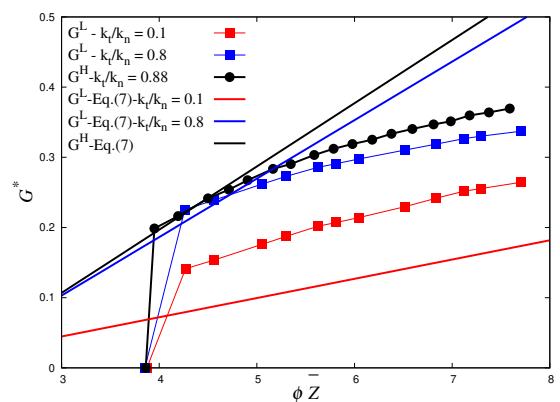

(b)

Figure 3.7: Normalized (a) bulk modulus $K^{*}$ and (b) shear modulus $G^{*}$ plotted against the product of volume fraction and coordination number $\phi \bar{Z}$ for samples with linear and Hertzian contacts. All samples are prepared without friction $\left(\mu=0\right.$ and $\left.k_{t}=0\right)$, and probed with different $k_{t}$ and $\mu=\infty$.

probing induces significant differences in the measured elastic moduli, with both bulk and shear moduli increasing with $k_{t}$. This is expected in the case of the shear modulus $G^{*}$, since tangential forces are activated during shear probing and the magnitude of the tangential stiffness plays an essential role. The effect of the tangential stiffness on $K^{*}$ is non negligible, even though if one would expect that mainly normal forces are activated during isotropic probing. The packings with Hertzian contacts show a bulk stiffness comparable with linear packings with a similar ratio $k_{t}^{L} / k_{n}^{L}=0.8$, and shear stiffness a little higher than the linear packings. The linear packings with small ratio $k_{t} / k_{n}$ have smaller and much smaller $K^{*}$ and $G^{*}$, respectively. In the same figures, the predictions from FT are plotted. The theory does not include a dependence on the tangential stiffness for the bulk modulus $K^{*}$ (see Eq. 3.7). That is why one line appears in Fig. 3.7(a), insensitive to $k_{t}$, that matches the numerical data with high $k_{t} / k_{n}$. The formulation of $G^{*}$ does involve a term related to $k_{t}$, and Eq. 3.7 is plotted in Fig. 3.7(b) for the two values of $k_{t}^{L} / k_{n}^{L}$ and for $k_{t}^{H} / k_{n}^{H}=0.88$. The agreement between FT and the numerical data is reasonable for $k_{t} / k_{n} \approx 0.8$, somewhat better for Hertzian contacts, as already discussed in Sec. 3.4. For low tangential stiffness, the FT captures qualitatively the DEM data of $G^{*}$, but with a large quantitative disagreement.

In order to quantify the effect of $k_{t}$ on the effective stiffness, we select a set of data at constant $\phi \bar{Z}=4.5$, then we subtract the reference values for the 
bulk and shear moduli, obtained with zero tangential stiffness, $K^{*}\left(k_{t}=0\right)$ and $G^{*}\left(k_{t}=0\right)$ from the moduli with finite $k_{t}$ and plot the data in Fig. 3.8 versus the ratio $k_{t} / k_{n}$. For both bulk and shear moduli, the effect of the tangential stiffness increases with $k_{t} / k_{n}$ and tends to saturate for higher values. The effect of $k_{t}$ on the shear modulus is larger than on the bulk modulus, due to high involvement of tangential forces during shear probing.

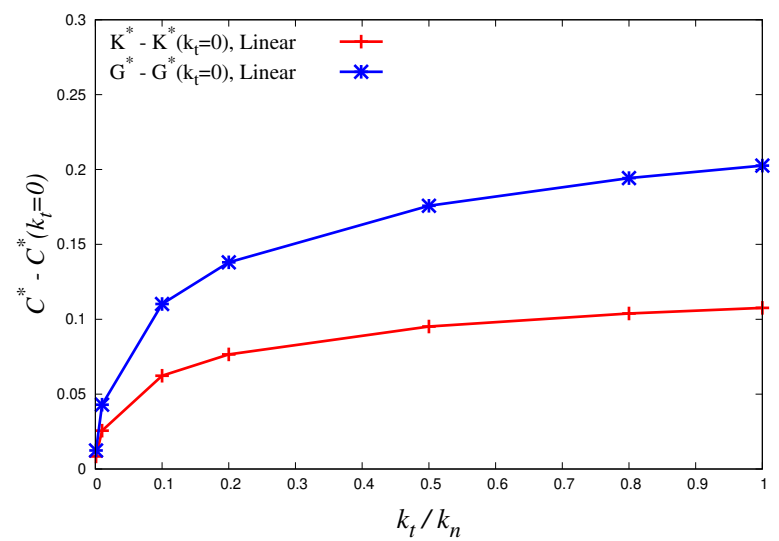

Figure 3.8: Dependence of the elastic moduli on the tangential stiffness for $\phi \bar{Z}=4.5$.

To further investigate this behaviour, we isolate the normal and tangential parts of the elastic moduli. To do this, we use a modified version of Eq. (3.2):

$$
\begin{array}{ll}
K_{\mathrm{N}}^{*}=\left.\frac{\Delta P^{* N}}{\Delta \varepsilon_{v}}\right|_{\Delta \varepsilon_{d e v}=0} & K_{\mathrm{T}}^{*}=\left.\frac{\Delta P^{* T}}{\Delta \varepsilon_{\nu}}\right|_{\Delta \varepsilon_{d e v}=0} \\
G_{\mathrm{N}}^{*}=\left.\frac{\Delta\left(\sigma_{x x}^{* N}-\sigma_{y y}^{* N}\right)}{2 \Delta\left(\varepsilon_{x x}-\varepsilon_{y y}\right)}\right|_{\Delta \varepsilon_{\nu}=0} & G_{\mathrm{T}}^{*}=\left.\frac{\Delta\left(\sigma_{x x}^{T}-\sigma_{y y}^{T}\right)}{2 \Delta\left(\varepsilon_{x x}-\varepsilon_{y y}\right)}\right|_{\Delta \varepsilon_{v}=0}
\end{array}
$$

where the stress components $\sigma_{i j}^{N}, \sigma_{i j}^{T}$, as well as $P^{N}, P^{T}$, are computed from Eq. (3.1) using only normal and tangential forces, respectively.

In Fig. 3.9, we plot the partitioned shear moduli versus $\phi \bar{Z}$ for packings with linear contacts and two values of tangential stiffness at probing, like in Fig. 3.8. We compare those with the partitioned shear response of Hertzian packings and 
the theoretical predictions from FT. The latter are obtained by Eqs. 3.7 considering only the terms proportional to $k_{n}$ and $k_{t}$; one line for $G_{N}^{*}$ is depicted in Fig. 3.9(a), while $G_{T}^{*}$ in Eq. 3.7 assumes different values for $k_{t}^{L} / k_{n}^{L}=0.1,0.8$, and $k_{t}^{H} / k_{n}^{H}=0.88$ in Fig. 3.9(b). The figures reveal several interesting features. In the case of linear contacts, the normal modulus $G_{N}^{*}$ increases in a similar fashion as in Fig. 3.7, while the tangential part $G_{T}^{*}$ stays almost constant close to zero for the whole $\phi \bar{Z}$ range. Surprisingly, the tangential stiffness influences mainly the normal partition of the shear modulus, rather than the tangential part. The major effect of $k_{t}$ during probing is not on the tangential forces, despite the direct link between these two quantities. In the case of Hertzian contacts, $G_{N}^{*}$ is higher than $G_{T}^{*}$, but with a ratio $G_{T}^{*} / G_{N}^{*} \approx 0.15$, meaning that the contribution of the tangential forces is distributed to both normal and tangential parts of the shear modulus. The normal shear modulus $G_{N}^{*}$ perfectly overlaps for linear and Hertzian contacts with similar $k_{t} / k_{n}$. Furthermore, the linear $G_{T}^{*}$ approaches the Hertzian data, the behavior of $G_{T}^{*}$ thus being at the origin of the differences between the two numerical cases as observed in Fig. 3.4(d). This comes from the fact that the tangential overlap is relatively smaller than the normal overlap; thus, the tangential force (tangential stress) is much smaller. Obtaining slightly higher $G_{T}^{*}$ in case of the Hertzian data comes from the fact that the Hertzian tangential stiffness depends on the normal overlap between particles. Hence, it is proportional to the change of the configuration $\left(k_{t}^{H} \propto \phi \bar{Z}\right)$, whereas, the linear tangential stiffness is constant throughout the simulations.

Furthermore, we want to assess the quality of theoretical predictions for the partitioned moduli and the dependence on the tangential stiffness. When we compare the Hertzian numerical data with the fluctuation theory, we observe underestimation of the theory for $G_{N}^{*}$. On the other hand, FT fails in describing the numerical $G_{T}^{*}$, with the mismatch becoming more dramatic for increasing tangential stiffness and $\phi \bar{Z}$.

The comparison between FT and DEM data is striking. For $G_{N}^{*}$, numerics and theory are far from each other, as the theory neglects $G_{N}^{*}\left(k_{t}\right)$. An opposite trend is seen for $G_{T}^{*}$ in Fig. 3.9(b) for the term proportional to $k_{t}$ in Eq. 3.7, i.e. $G_{T}^{*}$ vanishes when $k_{t}^{L} / k_{n}^{L}$ tends to zero; for $k_{t}^{L} / k_{n}^{L}=0.8$ the theoretical $G_{T}^{*}$ builds up while the DEM data stay practically unchanged, showing a dramatic difference with theoretical predictions (as well as with Hertzian data).

Despite FT includes an explicit dependence on $k_{t}$, the analytical prediction still neglects the effect of $k_{t}$ on $G_{N}^{*}$; the theory strongly overpredicts the material modulus due to the tangential forces. This is because the partitions $G_{N}^{*}$ and $G_{T}^{*}$ in Eqs. (3.4) and Eqs. (3.7) are associated only with the direct effect of $k_{t}$ on the contact forces (i.e. stress) while a more complex behaviour is possibly involved, 
due to corrections between non-affine displacements and tangential forces.

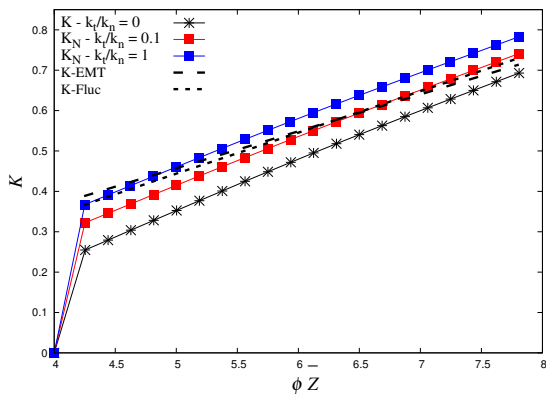

(a)

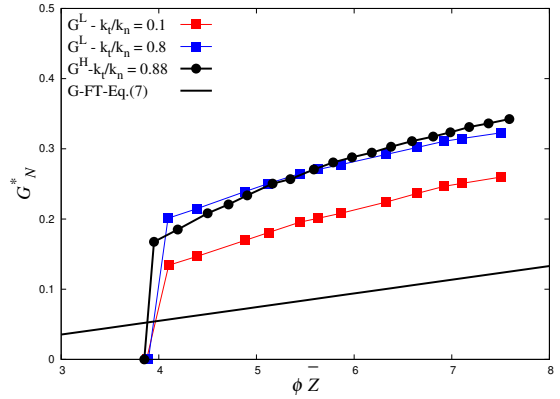

(b)

Figure 3.9: Normalized (a) normal shear modulus $G_{N}^{*}$, and (b) tangential shear modulus $G_{T}^{*}$ with volume fraction $\phi \bar{Z}$ for samples probed with tangential stiffness $k_{t} / k_{n}=0.1$ and 1 . Symbols correspond to DEM data, while lines are Eqs. (3.7), also partitioned in the normal and tangential components.

\subsubsection{Incremental non-affine fluctuations}

We want to investigate further the effect of the tangential stiffness on the contact network and the load/displacement redistribution during probing. We focus on isotropic probing and we measure the incremental fluctuations, ignoring the rotation term in Eq. 3.6, in the normal displacement of each pair of particles during probing as:

$$
\dot{\Delta}_{i}^{(B A)}=\dot{u}_{i}^{(B A)}-\dot{\varepsilon}_{i j} l_{j}^{(B A)}
$$

and define a measure of the average normal fluctuations over all pairs:

$$
\bar{\eta}=\frac{1}{M} \sum_{(B A) \in M}\left\|\frac{\dot{\Delta}^{(B A)}}{\dot{\boldsymbol{\varepsilon}} \boldsymbol{l}^{(B A)}}\right\| .
$$

In Fig. 3.10, we plot $\bar{\eta}$ versus $\phi \bar{Z}$ for packings with linear contacts and several values of $k_{t}^{L} / k_{n}^{L}$ as in Fig. 3.7, along with packings with Hertzian contacts. We compare $\bar{\eta}$ with the parameter $2 \rho$, that relates the average fluctuations in the normal displacement to contact statistics, as introduced in Eq. (3.8). 
All fluctuation terms $\bar{\eta}$ decrease with $\phi \bar{Z}$ as expected for more compact samples where particles movement is limited. Surprisingly, we found that the fluctuation parameter $\eta$ in packings with linear contacts is strongly affected by the tangential stiffness. $\eta$ assumes very high values close to jamming and decreases strongly with $\phi \bar{Z}$ for low tangential stiffness. For large tangential stiffness, the decay is much less pronounced and also the magnitude of $\eta$ decreases with $k_{t}$ increasing.

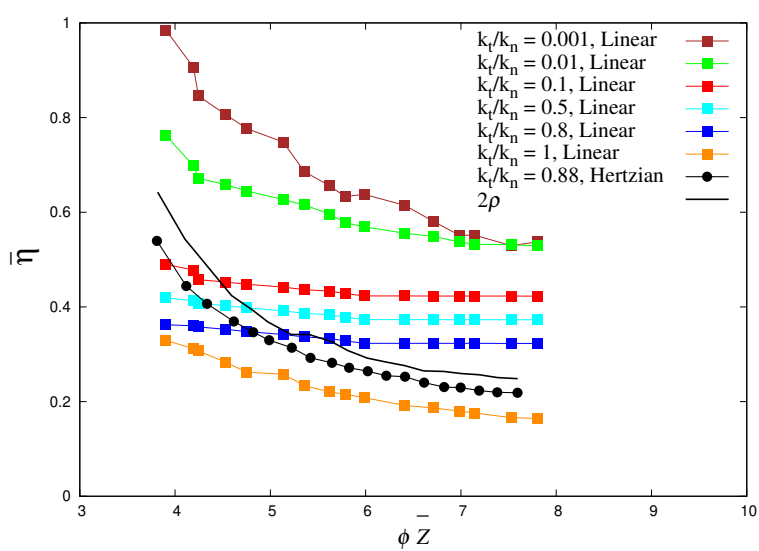

Figure 3.10: Displacement fluctuations of samples under isotropic compression plotted against the volume fraction for samples prepared frictionless and probed with different tangential stiffness.

$\bar{\eta}$ for Hertzian packings scales with $\phi \bar{Z}$ in a similar fashion. Hertzian packings (i.e. $k_{t}^{H} / k_{n}^{H}=0.88$ ) feature similar fluctuations as linear packings with $k_{t}^{H} / k_{n}^{H}=0.8$ but with around very different trends. On the other hand, weak tangential stiffness $k_{t}^{L} / k_{n}^{H}=0.1$ has a similar trend, but with double fluctuation intensity $\bar{\eta}_{k_{t}=0.1}^{L} \approx 2 \bar{\eta}_{k_{t}=0.88}^{H}$.

The contact fluctuations term $2 \rho$ remains constant when varying the tangential stiffness during probing, since we considered configurations prepared through the same protocol, i.e. with identical structure. Contact fluctuations as predicted by FT are in good agreement with values of displacement fluctuations measured from DEM Hertzian packings.

These observations hint at the importance of a particle fluctuation parameter for predicting the elastic behaviour of disordered granular systems. The data show that pairs of particles with linear contacts fluctuate in a different fashion 
than Hertzian contacts. Moreover, since it was systematically varied, we report that the displacement fluctuation term of linear contacts is highly dependent on the tangential stiffness $k_{t}$ but cannot state anything about the Hertzian case, since there we did not vary $k_{t}$.

\subsection{Concluding remarks}

This paper has made a contribution to advance the fundamental understanding of granular material by considering the effects of normal forces, inter-grain friction and tangential stiffness on the material response. Using Discrete Element Method (DEM) simulations of a triaxial cubical element test, we have studied the effects of preparation history, contact non-linearity and stiffnesses on the elastic moduli and granular elasticity. Major goal is to bridge between the micro-mechanical parameters and the macro-scale behaviour of granular materials.

The approach is a two-step one. First, we prepare three dimensional static packings of frictionless and frictional polydisperse particles at various confining stresses using DEM. Two popular contact models are compared, namely the linear visco-elastic and the non-linear Hertzian normal contact model with respective linear and non-linear tangential forces. Note that the samples have experienced different deformation history when the particles have different properties already during preparation. Second, the resulting packings were then analysed by studying their response to small strain perturbations.

In the cubical triaxial box, the elastic moduli were measured directly by applying different small strain modes, quantifying the incremental, elastic response of relaxed granular materials. Concerning this so-called probing, the amplitude of the applied perturbations had to be small enough to avoid particle rearrangements and thus get the true elastic response.

The effect of friction on the macroscopic elastic response is already considered at the end of preparation, over a wide range of confining pressures. Increasing friction between particles gives smaller elastic moduli at the same volume fraction (or density). We associate this to the less denser contact networks (smaller $\bar{Z}$ ) for lower $\mu$, established during preparation; this effect is more pronounced for the linear contact model, rather than for non-linear interactions. For the latter, correlations between the macroscopic elastic response and the microscopic were observed in the intermediate compression regime.

Furthermore, we focused on the effect of a varing tangential stiffness $k_{t}$ (associated with linear normal interactions) on the granular elasticity due to 
probing. Surprisingly, we found that the tangential stiffness strongly affects both the bulk modulus and the shear modulus, and even its normal component, where it is expected that mainly normal forces are active. When the tangential component is active during probing, the global force network becomes stiffer (at the same density) with reduced ability of the particles to move freely. The tangential part of the shear modulus is consistently very small, irrespective of the tangential stiffness, which leads to both qualitative and quantitative differences with the moduli measured in Hertzian granular assemblies.

Those aspects can have a major influence on the granular solid materials behaviour, but are not included in the existing theoretical frameworks, like the classical Effective Medium Theory (EMT), that attempts to link the elastic moduli to the packing microstructure. When the DEM data are compared with predictions from EMT, good agreement is found for the bulk modulus, but EMT fails to predict the shear modulus, as already reported [159, 160]. The fluctuation theory [93, 125] improves upon EMT and offers interesting insights. Concerning contact fluctuations are considered: $i$ ) the normal part of the shear modulus is well described, while specific features in the tangential part are not captured by the theory; $i$ i) the magnitude of fluctuations is very close to the values measured in DEM Hertzian simulations; $i$ i i) the influence of tangential stiffness on probing is not included in the theoretical framework.

Extension of the work to investigate the influence of inter-particle cohesion on the elastic moduli and structural anisotropy is in progress, using the same probing approach as in this paper. In addition, multi-contacts approach will be taken into account at high compression.

\section{Appendix A: Influence of contact slippage during probing}

In Sec. 3.3, samples were prepared with different inter-particle contact friction to obtain packings with different micro structural history. Then, small perturbations must be applied to the samples, to measure the elastic response. During probing, there are two possibilities for the friction coefficient between particles: ( $i$ ) either the friction coefficient remains unchanged, i.e., we keep the value as used for preparation, which may result in slippage; or ( $i$ i) it can be set to a much larger value to avoid sliding at the contacts (Sec. 3.3). Here, we discuss results on elastic moduli obtained by the two possibilities to see how important it is to set a proper friction coefficient between particles during probing. 


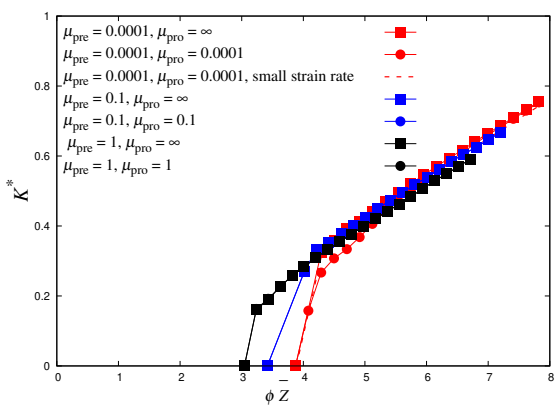

(a)

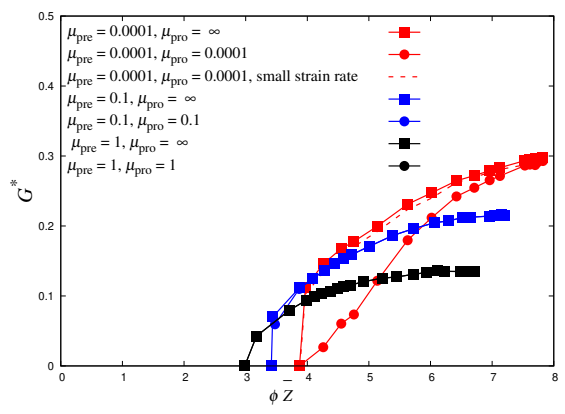

(b)

Figure 3.11: Normalized (a) bulk modulus $K^{*}$ and (b) shear modulus $G^{*}$ with $\phi \bar{Z}$ for different coefficients of friction, $\mu$, as shown in the legend. Dashed line corresponds to a case in which the friction coefficient $(\mu=0.0001)$ is kept unchanged during the preparation and probing but the applied strain is 1000 times slower than other cases.

In Fig. 3.12, the bulk and the shear modulus obtained using both methods are plotted for three different friction coefficients $\mu=0.0001,0.1$ and 1 against the product of volume fraction and coordination number. The elastic moduli deviate significantly for samples prepared with very small friction coefficient $\mu=0.0001$. This difference is more pronounced for samples at low volume fractions, which are less "rigid" and experience rearrangements already at very small strain. There is no significant difference between the two cases for $\mu=0.1$ and 1 . When the friction coefficient is set to a higher value with respect to the initial, preparation value, the increasing tangential forces can resist further motion in tangential direction, preventing rearrangements. This becomes of outmost importance in the case of low initial friction. Therefore, it is essential to set the friction coefficient $\mu$ to a large value to avoid sliding at the contacts when we apply small but finite strain perturbations in order to measure the actual elastic moduli of the granular assembly. However, one can keep the friction coefficient same as preparation by setting a much smaller strain amplitude during probing. Dashed line in Fig. 3.12 confirms that if the strain amplitude is set to a very small value, $\varepsilon_{\text {probe }} \leq 10^{-9}$ in this case, then it is not needed to set the friction coefficient to a large value to prevent the rearrangements. But, computational time of simulations will increase dramatically. When the strain amplitude is deduced by factor 100 or 1000, the results do not show 
dependence on $\mu$ during probing anymore.

\section{Appendix B: Notes on the Fluctuation Theory (FT)}

Incorporating fluctuations in the displacement of neighbours will result in a further relaxation of the kinematics and an improved prediction of the elastic moduli. Eqs. (B.1) are the solutions for the fluctuation of a typical pair of particles to be in equilibrium, assuming that the other particles in the neighbourhood move with the average strain.

$$
\begin{aligned}
& K^{*}=[1-2 \rho] \frac{\phi \bar{Z}}{3 \pi} D \\
& G^{*}=[1-2 \rho] \frac{\phi \bar{Z}}{5 \pi} D+\frac{3}{2} \frac{\bar{k}_{t}}{\bar{k}_{n}}[1-2 \rho+2 \zeta] \frac{\phi \bar{Z}}{5 \pi} D .
\end{aligned}
$$

Table B.1 provides values of parameters used in Eqs. B.1.

Fig. B.1 illustrates schematically a packing of polydisperse particles. For each pair the first ring of neighbours is defined. Particles in contact with the pair form a first-order sub-assembly. Those move following EMT in the basic FT Eqs. (B.1). When the second iteration is included in FT, pairs formed by the basic pair $A-B$ and particles in the first contacting ring also move with average plus fluctuations as was used in Eq. 3.7. For loose systems close to jamming a higher number of rings is needed to obtain a significant improvement as collective motion dominates the material behaviour $[4,116]$. Therefore, the theoretical framework could be extended further by involving more rings (see Eq. 3.7).

\section{Appendix C: system size - number of particles}

The number of particles of a simulation, i.e. the system size, can have big influence on the simulation results. Choosing a low number of particles may lead to bigger scatter in the outcomes. On the other hand, running simulations with a tremendous amount of particles leads to much more expensive computations. Furthermore, small samples might not be representative of the material behaviour, but bigger samples might develope inhomogenities or even shear bands. The main question here is whether the number of particles affects the elastic properties [120]. Therefore, probing was applied to configurations 


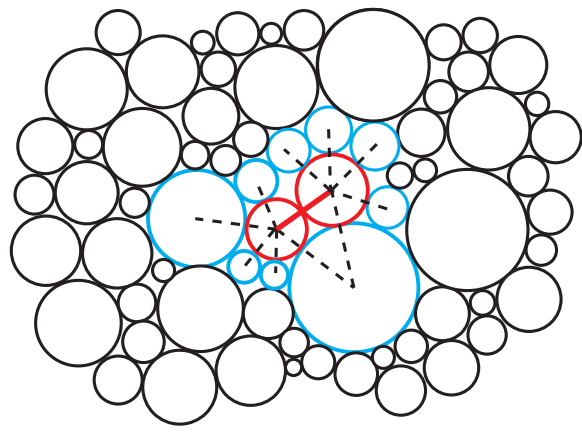

(a)

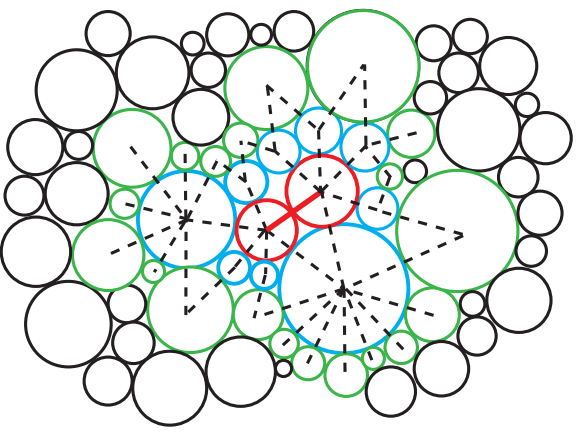

(b)

Figure 3.12: Sketch of neighboring assemblies for a specified central pair-particle shown in red. (a) First sub-assembly is shown by blue and (b) second sub-assembly is highlighted with green.

with different system sizes. Our aim was to determine the range of specimen sizes that yield identical results. For this reason, two system sizes were chosen $(N=1728,9261)$ along with the one used earlier $(N=4096)$. As expected, reducing the number of particles $(N=1728)$ induces undesired scatter. Tests with the larger number of particles $(N=9261)$ show no significant differences, which confirms the intermediate system size chosen in this research is large enough, yet not too large, to capture the macromechanical response with reasonable computational time.

\section{Acknowledgment}

We thank the anonymous reviewers who helped us to strongly improve the paper; we thank J.T. Jenkins, J-N Roux, and L. La Ragione for insightful discussions. Financial support for the study was provided by grant FP7 (ITN 607453) from the European-Union Marie Curie Initial Training Network, T-MAPPP, see http://www.t-mappp.eu/ for more information. 
74 Micromechanical study of the elastic stiffness in isotropic frictional granular solids

\begin{tabular}{|c|c|c|c|c|}
\hline$\phi$ & $\bar{Z}$ & $\chi$ & $\rho$ & $\zeta$ \\
\hline 0.5919819471 & 3.098039216 & 0.009213267105 & 0.1168783048 & -0.194781669 \\
\hline 0.5948472656 & 3.147058824 & 0.01266486156 & 0.1150246019 & -0.1952309987 \\
\hline 0.5977311055 & 3.059701493 & 0.005996430696 & 0.1182807852 & -0.1940782642 \\
\hline 0.6020919216 & 3.079545455 & 0.007720483109 & 0.1175603546 & -0.1944825855 \\
\hline 0.6064952609 & 3.147058824 & 0.01464465601 & 0.1152720762 & -0.1959953868 \\
\hline 0.6094547007 & 3.141732283 & 0.01391951056 & 0.1154285044 & -0.1958213927 \\
\hline 0.6124334262 & 3.108333333 & 0.01172293339 & 0.1167100317 & -0.1955826637 \\
\hline 0.61693802 & 3.210810811 & 0.01928374656 & 0.1129188443 & -0.1963428741 \\
\hline 0.6214868988 & 3.300947867 & 0.02539669942 & 0.1096137629 & -0.1961666792 \\
\hline 0.6245443565 & 3.48877551 & 0.04232656812 & 0.1035551934 & -0.1959423613 \\
\hline 0.62916826 & 5.152321981 & 0.09433859721 & 0.05443583724 & -0.1304492125 \\
\hline 0.6322762485 & 5.511873351 & 0.09924148489 & 0.04621852687 & -0.114392941 \\
\hline 0.6401362972 & 5.780095856 & 0.1006929941 & 0.04036548293 & -0.1021859473 \\
\hline 0.6481271691 & 6.057597367 & 0.0998437455 & 0.03445978849 & -0.08923188417 \\
\hline 0.6562515153 & 6.42972973 & 0.1026045645 & 0.02766033806 & -0.07429403919 \\
\hline 0.6645123002 & 6.677116827 & 0.1023512545 & 0.02323887315 & -0.06413070044 \\
\hline 0.6729123161 & 6.907964135 & 0.1062085761 & 0.0198589189 & -0.05662512297 \\
\hline 0.6814545088 & 7.133368065 & 0.1083798816 & 0.01656031324 & -0.04903708876 \\
\hline 0.6901418989 & 7.304504505 & 0.1121572771 & 0.01444546042 & -0.04432421065 \\
\hline 0.6989775848 & 7.509058433 & 0.1120092879 & 0.01146550102 & -0.03713717392 \\
\hline 0.7079647443 & 7.683180894 & 0.1120992238 & 0.009061436753 & -0.03130640746 \\
\hline 0.7171066373 & 7.819122099 & 0.1142846664 & 0.007512756695 & -0.0276964684 \\
\hline 0.7264066082 & 7.989706252 & 0.1152953295 & 0.0054281598 & -0.02264848908 \\
\hline 0.7358680888 & 8.126528575 & 0.1172260733 & 0.003953893991 & -0.01914929164 \\
\hline 0.7454946003 & 8.289793891 & 0.1173123615 & 0.001981545445 & -0.01424950946 \\
\hline 0.7552897567 & 8.418979187 & 0.1172534093 & 0.0004522572439 & -0.0104218977 \\
\hline 0.7652572673 & 8.551928783 & 0.1170006291 & -0.001103855714 & -0.006496608777 \\
\hline 0.7754009398 & 8.671597633 & 0.1168028459 & -0.002465948593 & -0.003049114738 \\
\hline 0.785724683 & 8.810591133 & 0.1167880368 & -0.003981255981 & 0.0007864348086 \\
\hline 0.7962325107 & 8.931510224 & 0.1165400074 & -0.005295320976 & 0.004141214911 \\
\hline 0.8069285443 & 9.039114391 & 0.1173829613 & -0.006306369481 & 0.006660510436 \\
\hline 0.8178170163 & 9.156963891 & 0.1168604495 & -0.007567892216 & 0.009919547323 \\
\hline
\end{tabular}

Table B.1: Coefficients used in FT obtained from a frictional packing $(\mu=1)$. 


\section{Chapter 4 \\ Elastic waves in particulate glass-rubber mixtures}

Yesterday I was clever, so I wanted to change the world. Today I am wise, so I am changing myself.

Rumi

In this paper, we study the propagation of waves in dense static granular packings made of soft and stiff particles subjected to different hydrostatic stresses. We perform physical experiments in a triaxial cell equipped with piezoelectric wave transducers. Mixtures made of monodisperse glass and rubber beads are prepared at various species fractions. Low-frequency waves are propagated through the samples and the time of flight is measured in order to shed light on the combined effect of applied stress and rubber content on the elastic properties of the mixtures. Interestingly, the bulk stiffness deduced from the wave speed shows that the behaviour is non-linear and non-monotonic with the increasing percentage of rubber particles, the effect being more prominent at higher pressures. The modulus remains fairly constant (slightly increased relative to $100 \%$ glass) when increasing the fraction of rubber to $30 \%$, while they experience a sudden drop between $30 \%$ and $60 \%$, to become again constant between $60 \%$ to $100 \%$. Along with the stiffness investigation obtained from the time domain signal, a Fast Fourier Transform analysis gives insights into the frequency domain, showing the transition from glass- to rubberdominated media. Finally, the influence of the rubber particles on the dissipation 
of samples at low rubber content is addressed. Mixtures with rubber content below $30 \%$ show enhanced damping properties, associated with slightly higher stiffness and lighter weight. ${ }^{1}$

\subsection{Introduction}

The behavior of particulate mixtures is of interest for a large number of fields, materials, and applications, including sintering, ceramics, gels, mineral processing, pharmaceutics, environment engineering, and geomechanics.

In geotechnical engineering, it is common to incorporate recycled materials (e.g. shredded or granulated rubber, crushed glass) into earth constructions for conventional designs and soil improvement projects [20,64, 134]. Similarly, mixtures of asphalt and concrete are widely used to construct roads [51, 267]. Exploring the effect of granular composition on the effective physical properties of mixtures can help optimizing industrial processes, and engineering (infra) structures $[85,188,267,274]$. The topic has received increasign attention in the last years $[53,108,133,135,170,251]$. An area of particular interest is the response of mixtures to the propagation of elastic waves, as related to the density of light and soft components to damp high amplitude waves. Despite the massive amount of work on granular mixtures, a deep understanding from a micromechanical perspective is still missing. This is because the role of the different components can hardly be discerned by classical experiments.

In early experimental works $[53,133,135,170,251]$, the authors have shown that the velocities of propagating shear waves in binary sand-rubber mixtures scale non-linear and non-monotonic fashion with the increasing volume fraction of rubber chips. A similar behavior was observed more recently in Ref. [236] for longitudinal waves in binary mixtures of glass and rubber beads of equal diameter.

Although several methods are commercially available to determine the stiffness of geomaterials both in the laboratory and in the field, the wave propagation techniques are widely accepted for their rapid, non-destructive, and low-cost evaluation methods. The use of piezoelectric transducers to estimate small-strain stiffness of soils from wave velocity in the laboratory has been well established nowadays [27, 96, 159, 186, 208, 210, 261]. Along with the characterization of the material bulk stiffness, wave analysis provides information

\footnotetext{
${ }^{1}$ Taghizadeh, K., Steeb, H., Luding, S., \& Magnanimo, V. Journal of Mechanics and Physics of Solids, submitted Aug. 2019.
} 
about the attenuation properties of the medium, i.e. the energy that the elastic waves loose during traveling.

On the other band, the quality factor is a prominent parameters having an important effect upon the amplitude and duration of ground motions during earthquakes $[95,99,101,188]$. Its determination appears to be a crucial point for the quantitative interpretation of the amplification effects often produced by surficial deposits [52].

In this study, we apply wave technique to study stiffness and attenuation in granular materials made of glass and rubber beads. The focus is on the effect of mixture composition and pressure level where these factors can be exploited to optimize the material behavior.

This paper is organized as follows: Sec. 2 describes the details of the experimental procedure; we show the elastic stiffness of mixtures in sec. 3 ; frequency analysis of different mixtures are shown in sec. 4; in sec. 5, we compute the quality factor of particular samples using of spectral ratio method. Finally, sec. 6 concludes the paper.

\subsection{Experiments}

\subsubsection{Test procedure}

The experimental methodology is described in this section. Glass and rubber particles with similar size $\left(d_{r}=d_{g}=4 \mathrm{~mm}\right)$ are used in this study to prepare cylindrical specimens with different volume fractions of glass and rubber beads. The material characteristics for both glass and rubber beads are reported in Table 4.1.

To obtain the particle stiffness of glass and rubber, we performed a compression test on single particles. The force-displacement curve of glass and rubber is plotted in Fig. 4.1. A Hertzian law well fits the data in the pressure regime considered [187, 194]. Starting from the fitting curves, the Young's modulus for glass and rubber particles can be deduced.

\begin{tabular}{lll}
\hline Used material properties & Glass & Rubber \\
\hline Diameter $(\mathrm{mm})$ & 4 & 4 \\
Mass density $\left(\mathrm{kg} / \mathrm{m}^{3}\right)$ & 1540 & 860 \\
Young's modulus $(\mathrm{MPa})$ & 754000 & 600 \\
\hline
\end{tabular}

Table 4.1: Properties of glass and rubber particles. 
Let $v$ be the ratio between the volume of rubber particles and the total volume of solids in the mixture. Glass-rubber samples were prepared with variable rubber content, $v=0,0.1,0.2, \ldots, 0.9,1.0$, where $v=0$ is composed of glass particles only and $v=1.0$ of rubber particles only. Care is taken to prepare homogeneous mixtures and avoid segregation by minimizing vibration during specimen preparation. All specimen are tested in our custom-made triaxial cell with the sample diameter equal to $100 \mathrm{~mm}$ (Fig. 4.2.a). Two sample height are considered in this study, 100 and $70 \mathrm{~mm}$.

The instrumentation system consists of a pair of piezoelectric transducers, function generator, signal amplifier, voltage divider for the input signals and digital oscilloscope, signal amplifier/filter and digital oscilloscope. Fig. 4.2.b shows a schematic drawing of the set up and peripheral electronics. Granular samples are compressed in the axial direction via the top piston in subsequent stress increments. At each step the radial stress is corrected to match the axial stress. Water is used as confining fluid for the samples enclosed by a rubber membrane. Ten pressure values are analyzed, between $P=50 \mathrm{kPa}$ and $500 \mathrm{kPa}$. At each pressure level, a high voltage burst signal is excited to measure the time of flight. The top cap (the sound source) and the bottom plate (the detector) of the cell are instrumented with piezoelectric transducers with a diameter adjusted via a PMMT contact plate to the sample size, able to excite longitudinal

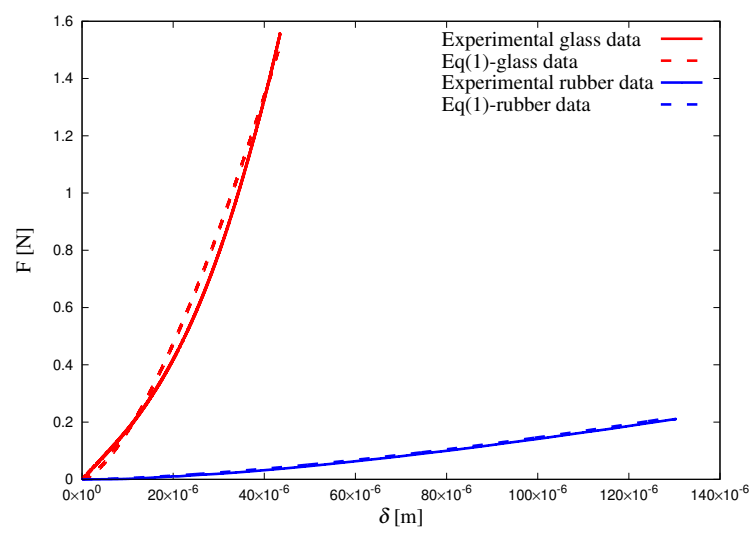

Figure 4.1: Experimental (solid line) force-displacement plot of a single particle compression fitted by a non-linear Hertzian law (dashed line). Red and blue color indicate glass and rubber particles with properties given in Table 4.1, respectively. 
waves and measure the arrival time, respectively.

We limit our studies to the compressional P-waves. The transmitted signal is a $\pm 400 \mathrm{~V}$ step signal via the top cap. The signals transmitted and received are pre-amplified, filtered and recorded with a digital oscilloscope (LeCroy WaveSurfer $1 \mathrm{GHz}$ ). The signal-to-noise ratio is improved by repetitive averaging of 100 detected signals using the digital oscilloscope and then sent to a computer for further processing. We repeat the experiment five times for each rubber content and pressure level in order to avoid configuration-dependent results.

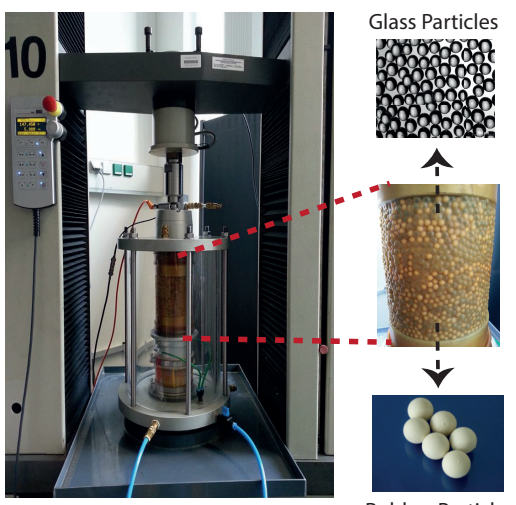

(a)

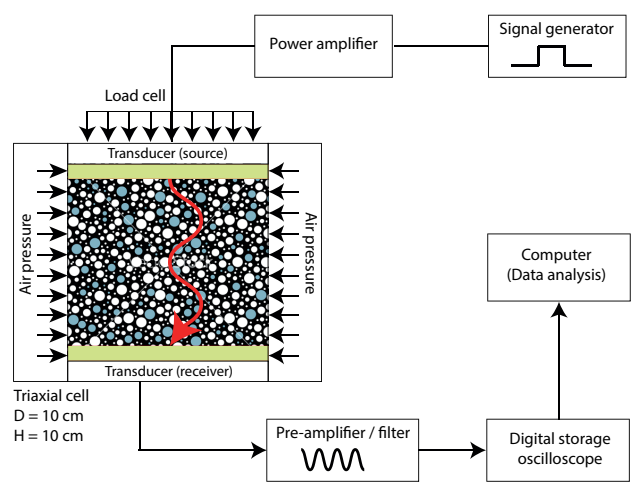

(b)

Figure 4.2: (a) Acoustic triaxial cell with piezoelectric transducer measurement system and a prepared sample with glass (dark) and rubber (light) particles. (b) Schematic drawing of the experimental setup.

\subsubsection{Mixture properties}

For each rubber content $v$, the mass density of the sample is given by

$$
\rho_{B}=(1-v) \rho_{g}+v \rho_{r}
$$

where $\rho_{g}$ and $\rho_{r}$ are true mass densities of glass and rubber beads, respectively. The given expression can be rewritten in terms of number of glass and rubber particles as

$$
\rho_{B}=\frac{V^{p}\left(\rho_{g}^{p} N_{g}+\rho_{r}^{p} N_{r}\right)}{V_{b o x}},
$$


where superscript $p$ denotes a single particle parameter, then $V_{p}$ becomes the single particle volume and $V_{\text {box }}$ is the volume of the sample, $V_{\text {box }}=\frac{\pi}{4} D^{2} \mathrm{H}$, with $D=100 \mathrm{~mm}$ diameter of the sample and $H=100$ or $70 \mathrm{~mm}$ height of the sample in the two cases considered. $N_{g}$ and $N_{r}$ represent the number of glass and rubber particles used in each experiment. Since samples are made of monodisperse glass and rubber particles, a relation between rubber fraction and number of particles can be found as

$$
(1-v) N_{r}=v N_{g},
$$

and the number of glass and rubber particles in each experiment can be estimated by Eqs. 4.2 and 4.3. Knowing the volume of the cylinder before and after deformation, one can measure the sample volume fraction by

$$
\phi=\frac{\left(N_{g}+N_{r}\right) V^{p}}{V_{b o x}} .
$$

In Fig. 4.3, we plot the mass density versus rubber content and the volume fraction against applied pressure. Rubber is softer than glass and can deform easily, filling the pores. Therefore, the volume fraction increases by increasing both, pressure and rubber content. Adding rubber particles makes the system softer. Thus, the volume fraction becomes sensitive to the change of pressure with the slope of the lines getting steeper for high rubber contents.

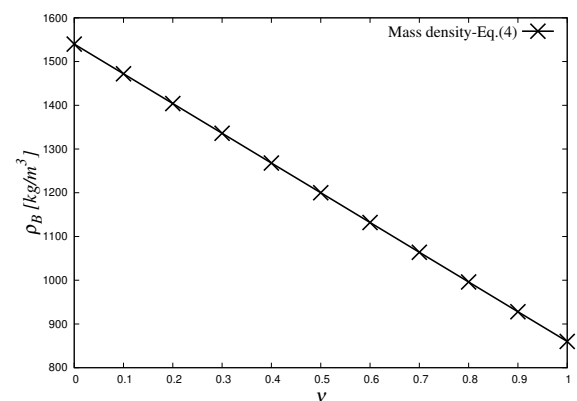

(a)

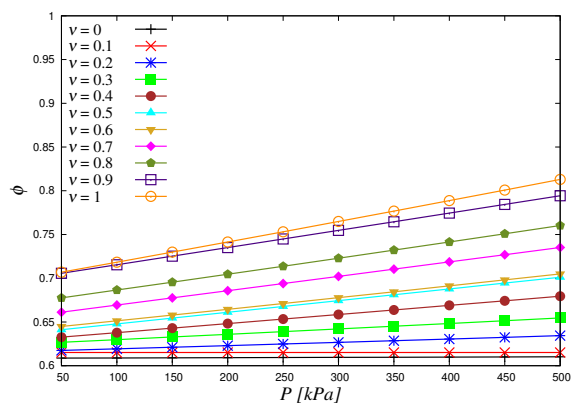

(b)

Figure 4.3: a) Mass density of samples at different rubber content. b) Measured volume fraction of samples during loading at different pressure for samples with different mixtures. 


\subsection{P-wave velocity}

For each rubber content and pressure level, waves are propagated using a step input signal with amplitude of $400 \mathrm{~V}$ and the time of flight of the wave through the sample is measured. A typical output is presented in Fig. 4.4 for a sample with rubber content $v=0$ and pressure $P=300 \mathrm{kPa}$. There is significant uncertainty and difficulty associated with determining the travel time (see Appendix A). Suggested criteria and recommendations vary depending on the installation, application and input signal. Here, we adopt a consistent peak-topeak approach.

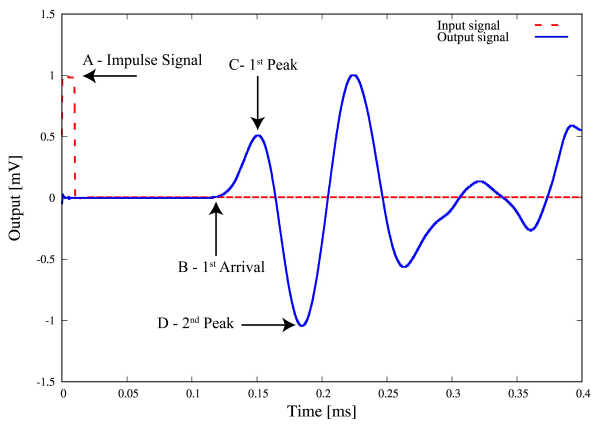

Figure 4.4: Typical input and output signals from the transmitting and receiving piezoelectric transducers.

Fig. 4.5 shows the P-wave signals recorded during pressure loading and unloading for several rubber contents. Looking at these signals, it is clear that the waveforms are sensitive to changes in material composition and confining stress. For samples prepared at low rubber content $(v=0,0.1$, to 0.3$)$, the waveformes show a clear peak followed by a deep valley, that moves to the left on the time axis with increasing pressure. The response for $v=0.3$ still shows pronounced peaks, with the position in time related to pressure. However, additional features in the coda start to appear associated with high frequency transmission, especially for high pressure levels. At $v=0.5$, the behavior resembles that of lower rubber contents only at high pressure, while at low pressure a new peak, later in time, appears, weakly associated with those at $v<0.5$, and high frequency ripples are visible in both the peak and post-peak parts. For $v \geq 0.7$, the pressure-dependent peak disappears and high frequency features dominate. Comparison between $v=0.3,0.5$, and 0.7 clearly shows a pronounced change 
in waveforms associated to a transition from stiff to soft dominated regimes. Adding soft particles to the sample, not only leads to delay in events but also to additional features in the post-peak part of the signal. Furthermore, waveforms of $v=0.7$ and 1 reveal that soft dominated samples are insensitive to pressure.

By measuring the travel time of the P-wave $\left(t_{p}\right)$ and the tip-to-tip distance between transmitting and receiving transducers $(L)$, the P-wave velocity in the specimen $\left(V_{p}\right)$ is obtained as

$$
V_{p}=\frac{L}{t_{p}}
$$

Fig. 4.6.a and 4.6.b show the wave velocity versus rubber content and pressure. The wave transmission velocity remains fairly constant up to $v=0.3$. By increasing the volume of rubber, there is a considerable drop in the wave velocity for all pressure levels, which can be associated to the transition from glassto rubber-dominated regimes. The wave velocity is again relatively stable for $v=0.7$ to 1 , where the medium is, eventually, controlled by rubber particles.

In the long-wavelength limit, the longitudinal P-wave modulus, $M$, is related to the velocity, $V_{p}$, by

$$
M=\rho_{B} V_{p}^{2},
$$

where $\rho_{B}$ is the mixture's bulk density of the sample as given by Eq. 4.1.

Fig. 4.7.a shows the evolution of the bulk stiffness with rubber content at different pressure levels. The compressional modulus shows high stiffness for rubber content up to $v=0.3$. In the case of high pressure, adding a small amount of soft particles surprisingly enhances the effective stiffness of the medium and the highest modulus is observed at $v \approx 0.2$. Between $v=0.3$ and 0.6 , there is a considerable drop in the wave velocity which highlights the transition from glass- to rubber-controlled media. The modulus is again relatively stable between $v=0.6$ to 1 , which is linked to the dominance of the rubber media.

In Fig. 4.7.b, the $M$-modulus is plotted against pressure $P$. The slopes of the $M$-lines with $P$ are almost constant for $v \leq 0.5$. Only the sample with $v=0$ shows a slightly smaller slope $m \simeq 0.33$, in agreement with theoretical expectations $[70,155,159,229]$. The behavior suddenly changes when the rubber content is increased from $v=0.5$ to 0.6. Further on, at higher rubber contents, the modulus becomes almost independent of pressure. We associated the change in the material behavior to the transition from a glass dominated regime, where waves travel through glass-glass contacts, to a rubber dominated case [17], 


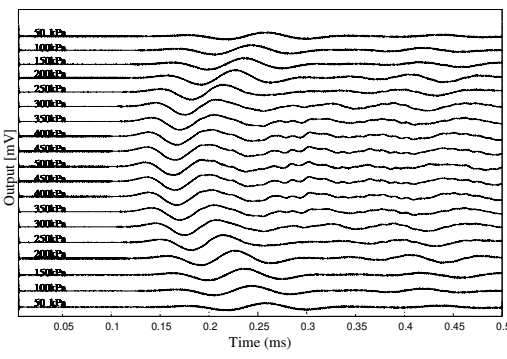

(a)

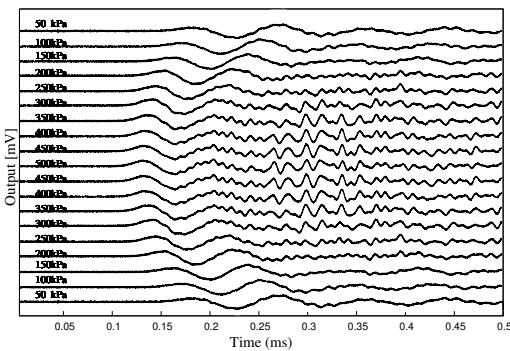

(c)

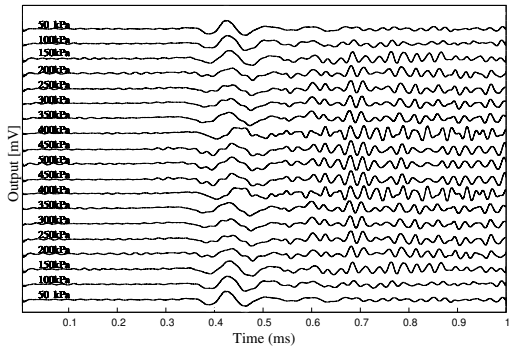

(e)

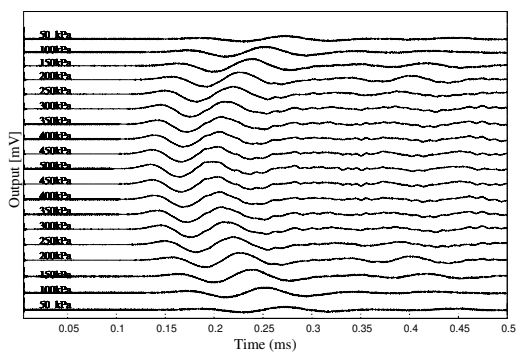

(b)

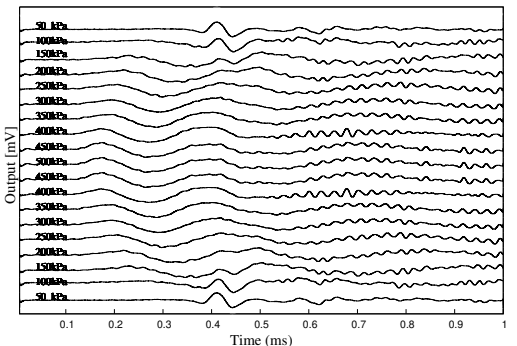

(d)

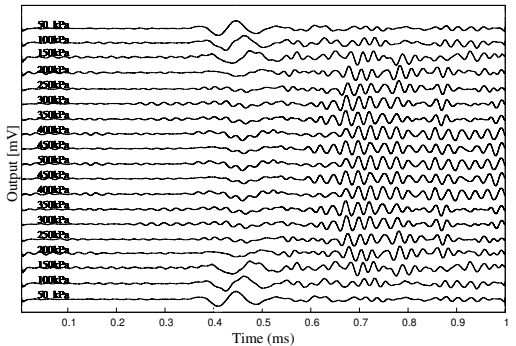

(f)

Figure 4.5: Received P-wave signal series during loading (from 50 to $500 \mathrm{kPa}$ ) and unloading (from 500 to $50 \mathrm{kPa}$ ) for samples with different rubber content, (a) $v=0$, (b) $v=0.1$, (c) $v=0.3$, (d) $v=0.5$, (e) $v=0.7$, and (f) $v=1$. Note that the limit of $\mathrm{x}$-axis varies for different plots for the sake of visibility. It is considered $0.5 \mathrm{~ms}$ and $1 \mathrm{~ms}$ for $v \leq 0.3$ and $v \geq 0.3$, respectively. 


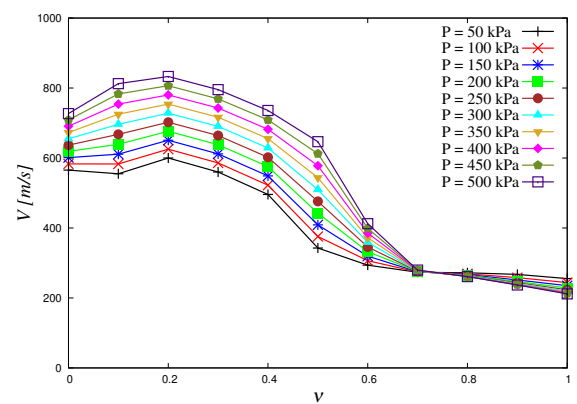

(a)

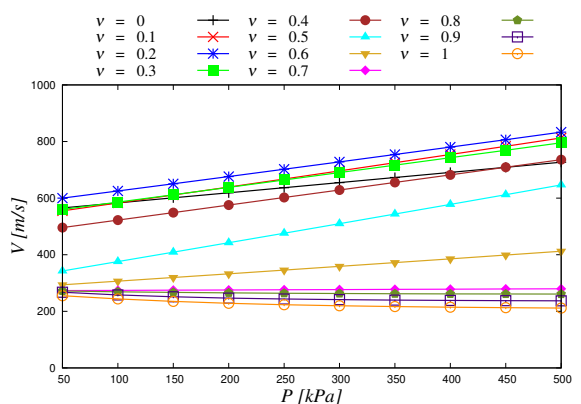

(b)

Figure 4.6: a) P-wave velocity against fraction of rubber. b) P-wave velocity against confining stress.

where the packing seemingly reasonable on homogeneous rubber block [12, 13].

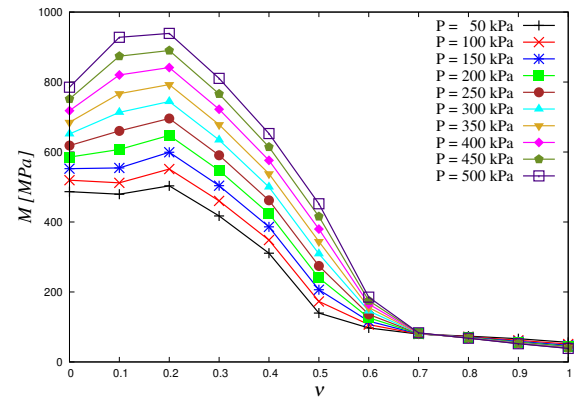

(a)

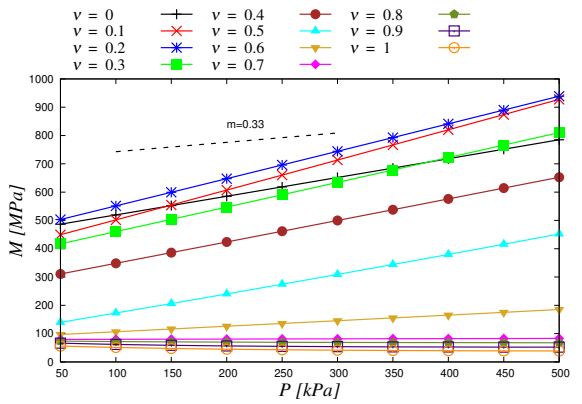

(b)

Figure 4.7: a) P-wave modulus against fraction of rubber. b) P-wave modulus against confining stress. For reference, lines with slope $m=0.33$ (dashed) is shown. 


\subsection{DEM study}

\subsubsection{Numerical setup}

To understand the experimental observations we perform numerical simulations and try to reproduce the behavior glass-rubber mixtures tested in the experimental part [53]. The Discrete Element Method (DEM) allows to simulate large number of interacting particles that either move following Newton's second law or just in static mechanical equilibrium (before the wave-tests) [150]. Given two elastic spheres $i$ and $j$ of radii $R_{i}$ and $R_{j}$ at position $\vec{x}_{i}$ and $\vec{x}_{j}$, the contact force in the normal direction is given by the Hertz contact law, by

$$
F_{n}=\frac{4}{3} E^{*} R^{1 / 2} \delta^{3 / 2}+F_{d},
$$

where $R=R_{i} R_{j} /\left(R_{i}+R_{j}\right)$ is the effective radius, $\delta=\left[R_{i}+R_{j}-\left|\vec{x}_{i}-\vec{x}_{j}\right|\right]$ is the normal overlap and $E^{*}$ is the effective modulus

$$
\frac{1}{E^{*}}=\frac{1-v_{i}^{2}}{E_{i}}+\frac{1-v_{j}^{2}}{E_{j}},
$$

with $E_{i}, E_{j}$ and $v_{i}, v_{j}$ Young's moduli and Poisson's ratio, respectively. $F_{d}$ is the damping force between two particles and given by

$$
F_{d}=\gamma \sqrt{d \delta} \dot{\delta}
$$

where $\gamma$ is the Hertzian viscosity parameter and $\dot{\delta}$ is the relative velocity of two particles. Note that $F_{d}$ contributes very small value (almost zero) in case of quasi-static simulations since $\dot{\delta} \approx 0$ (particles do not move).

In a tangential direction a linear-dashpot contact model with Coulumb threshold is used with stiffness $k_{t}$ and damping $\gamma_{t}$ as defined in [142, 233] and with coefficient of particle friction $\mu=\mu_{s}=\mu_{d}$. To find the inter-species parameters (numerical value of parameter between a glass and a rubber particle), the reciprocals of parameters are added and the reciprocal of the sum is taken (product over sum) as the inter-species numerical value. For example, inter-species density between glass and rubber particles is given by $\rho_{g, r=\frac{\rho_{g} \rho_{r}}{\rho_{g}+\rho_{r}}}$, note that other contact properties follow the same rule. For glass and rubber particles the material characteristics in Table 4.1 and contact properties in Table 4.2 are used. 
Elastic waves in particulate glass-rubber mixtures

After defining the microscopic interaction between grains, we describe the protocol to generate packings and measure the elastic moduli. We start our simulations from a set of non-overlapping particles randomly generated in a periodical cubic box at an initial volume fraction $\phi=0.3$. The initial configuration is compressed isotropically by constant compression strain-rate until a given volume fraction $\phi=0.5$, below the jamming point. The system is then allowed to relax at constant volume fraction until it reaches a stable state, which means that the particles dissipate kinetic energy and achieve a zero-pressure unjammed relaxed configuration. This is followed by an isotropic compression until the desired maximum volume fraction, $\phi=0.82$ [121]. The same protocol is used for all glass-rubber mixtures from $v=0.0$ to 1.0. In this way, samples resembling the set of experiments to some extent in Sec.2 are created. In simulations the preparation is isotropic, periodic without walls all the time, whereas in experiments, only hydrostatic stress conditions have been applied, thus isotropy of packing could not be guaranteed.

\begin{tabular}{llll}
\hline Property & Symbol & Value & SI-units \\
\hline Time unit & $t$ & 1 & $10^{-6} \mathrm{~s}$ \\
Length unit & $x$ & 1 & $10^{-3} \mathrm{~m}$ \\
Mass unit & $m$ & 1 & $10^{-9} \mathrm{~kg}$ \\
Particle radius & $\langle a\rangle$ & 2 & $10^{-3} \mathrm{~m}$ \\
Number of particles & $N$ & 5000 & \\
Particle density, g & $\rho_{g}$ & 2540 & $2540 \mathrm{~kg} / \mathrm{m}^{3}$ \\
Particle density, r & $\rho_{r}$ & 1270 & $1270 \mathrm{~kg} / \mathrm{m}^{3}$ \\
Simulation time step & $\Delta t_{M D}$ & 0.01 & $10^{-8} \mathrm{~s}$ \\
Tangential stiffness, g & $k_{t}^{g}$ & $10^{4}$ & $10^{7} \mathrm{~kg} / \mathrm{s}$ \\
Tangential stiffness, $\mathrm{r}$ & $k_{t}^{r}$ & 150 & $150 \cdot 10^{3} \mathrm{~kg} / \mathrm{s}$ \\
Viscosity, g & $\gamma_{g}$ & 100 & $100 \mathrm{~kg} / \mathrm{s}$ \\
Viscosity, r & $\gamma_{r}$ & 100 & $100 \mathrm{~kg} / \mathrm{s}$ \\
Friction coefficient & $\mu$ & 0.5 & \\
\hline
\end{tabular}

Table 4.2: Summary and numerical values of particle parameters used in the DEM simulations [142]

Once packings are created and compressed, various configurations are picked up at different pressure states above the jamming volume fraction. Those samples are allowed to relax with constant volume until a stable state is reached, which means the pressure remains unchanged over a period of time. Then a small strain perturbation is applied to these relaxed samples, either pure volumetric or pure deviatoric $[155,233]$. The bulk and shear moduli $K$ and $G$ 
are calculated as the ratio between the measured increment in stress and the applied strain:

$$
\begin{aligned}
K & =\frac{\delta P}{3 \delta \varepsilon_{v}}=\frac{\delta\left(\sigma_{x x}+\sigma_{y y}+\sigma_{z z}\right)}{3 \delta\left(\varepsilon_{x x}+\varepsilon_{y y}+\varepsilon_{z z}\right)}, \\
G_{x y} & =\frac{\delta\left(\sigma_{x x}-\sigma_{y y}\right)}{\delta\left(\varepsilon_{x x}-\varepsilon_{y y}\right)}, \text { with } \delta \varepsilon_{z z}=0,
\end{aligned}
$$

where $\sigma_{i j}$ and $\varepsilon_{i j}$ are components of stress and strain tensors respectively. Since the aggregates are isotropic, the P-wave modulus is given by:

$$
M=K+\frac{4}{3} G,
$$

as also measured directly by $M \simeq \frac{\delta \sigma_{x x}}{\delta \varepsilon_{x x}} \simeq \frac{\delta \sigma_{y y}}{\delta \varepsilon_{y y}} \simeq \frac{\delta \sigma_{z z}}{\delta \varepsilon_{z z}}$ when only one strain is activated.

\subsubsection{Numerical results}

In this section we show the results of the numerical moduli for different rubber content $v$ and pressure states. Note that a pressure range in simulation is wider than in experiments to gain more insight into the mechanical response of mixtures. In Fig. 4.8 we plot the P-wave moduli versus pressure, decreasing monotonically with the rubber content from $v=0.0$ to $v=1.0$. In this respect, simulations are not able to reproduce the macroscopic behavior observed in Fig.4.7 in the experiment the maximum $M$ at $v \neq 0$. We associate the mismatch to the adopted contact model not appropriate to describe rubber-rubber and rubber-glass interactions. Finding a better contact model is subject to ongoing study. However, when experiments and simulations are directly compared in Fig.4.9, interesting information can be inferred. For the sake of clarity, only three cases are shown, namely, $v=0.05, v=0.5$ and $v=1.0$. Simulations with $v=0.05$ capture the experimental data quantitatively, noticeably without any calibration. On the other hand, when looking at the packing $v=0.5$, the qualitative trend is well captured by simulations even if actual experimental values are higher than the simulated ones. Finally, for the case $v=1.0$, where the moduli are pressure independent, simulations are far from experiments in qualitative trend.

By summarizing the previous observations, a three regime scenario shows up. In the glass-dominated regime $\mathrm{G}$, waves do transmits via a glass beads network, 


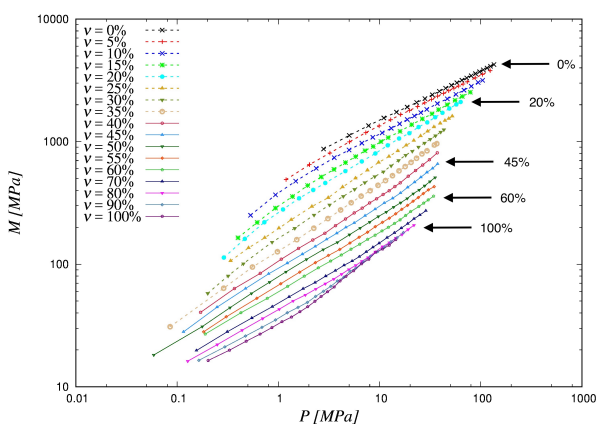

Figure 4.8: P-wave modulus versus applied vertical stress obtained by DEM simulations.

where simulations based on Hertzian interactions are able to reproduce the macroscopic behavior. In the intermediate regime I, waves still have a preferential path via glass bead chains. Here two mechanisms concur to shape the bulk behavior: i) the density of glass beads in the sample reduces with respect to case $\mathrm{G}$ and the actual values of the moduli get lower; ii) the number of contacts increases with pressure faster than in the G-regime due to easy rearrangement of the rubber particles, that is the slope $M(P)$ gets higher. Finally, in the third regime $\mathrm{R}$, the behavior of the mixture is dominated by the rubber beads, and the present simple DEM contact model can not offer an accurate representation of the system.

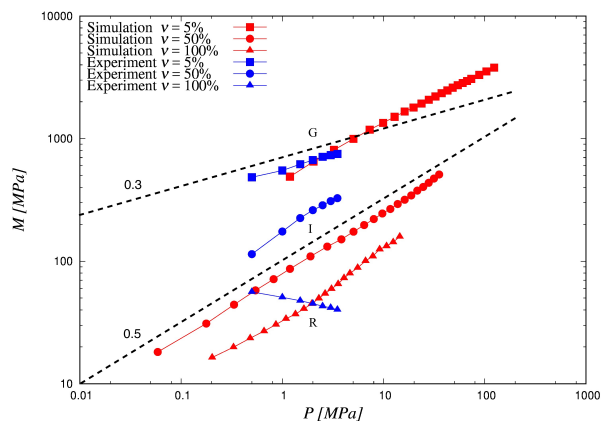

Figure 4.9: Experimental and numerical P-wave modulus plotted against pressure; comparison of DEM and experimental glass-rubber mixtures for $v=0.05,0.5$ and 1.0 . 


\subsection{Frequency spectrum}

In order to understand the transition in the material behavior as observed in Figs. 4.5, 4.6, and 4.7, we study the previous samples in the frequency domain by applying a Fast Fourier Transform (FFT) to signals obtained by experiments [77]. We focus on the first wavefront that determines the wave velocity (and sample stiffness) and discard the coda. It is worth mentioning that filtering function has not been applied to not lose any information.

From the FFT of a signal is possible to derive the amplitude $A$ for a given frequency $f$ and thus the energy (power) parameter $|A(f)|^{2}$. In Fig. 4.10.a, the energy is plotted for samples with rubber contents of $v=0,0.1,0.2$, and 0.3 at two different confining pressures $P=200$ and 500kPa. For these low rubber contents, the main frequency remains unchanged despite the increase in the effective stiffness (see Fig. 4.7). The dominant frequency does not change with the confining pressure, only the energy increases as the specimen is subjected to higher confining pressures.

Furthermore, we focus on the frequency response in the transition regime. Fig. 4.10.b shows the energy plot against frequency for samples at $v=0.4,0.5$, and 0.6 rubber content and two pressure levels $P=200$ and $500 \mathrm{kPa}$. For $v=0.4$, the main frequency is close to the value obtained for the cases $v \leq 0.3$. However, a second peak start to appear at higher frequency. Samples with $v=0.5$ show two peak frequencies, one close to the value seen before, related to glass driven propagation, and another, very wide at $f=0.055 \mathrm{MHz}$. While the energy associated with $v=0.5$ at $P=200 \mathrm{kPa}$ is low and widely spread, at $P=500 \mathrm{kPa}$ becomes clear that the high frequency signal dominates, with a low amplitude, low frequency peak still surviving. The trend becomes even more pronounced for $v=0.6$, where the low pressure sample shows almost no peak (with the energy possibly stored in the coda) and a bimodal behaviour appearing at high pressure, around $f=0.055 \mathrm{MHz}$. For these intermediate rubber contents, the wave first arrival (see Fig. 4.5.d) seems to be related with the glass network, while the energy is mainly concentrated at higher frequencies.

Finally, rubber contents $v=0.7$ and 1 are depicted in Fig. 4.10.c. Unlike the earlier plots, this figure does not show any significant frequency at $0.01 \mathrm{MHz}$ which was associated with glass particles, but the energy is concentrated at 0.05 $\mathrm{MHz}$, that is the peak frequency of the pure rubber media. Therefore, it can be concluded that glass particles do not play an important role in samples with high rubber contents.

It is worth mentioning that the FFT analysis has been applied on the input signal too. As expected, the energy is very small in comparison to the output 
signals which ensures that samples have not been burst by the input.

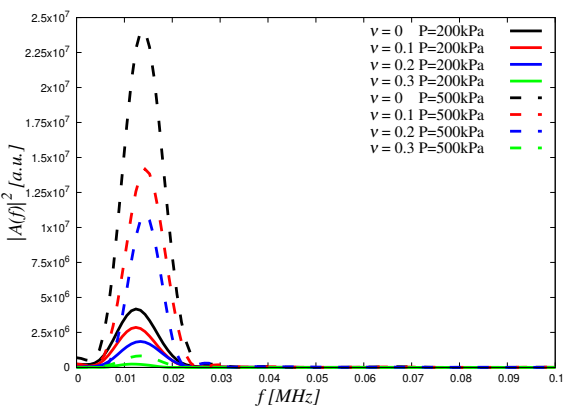

(a)

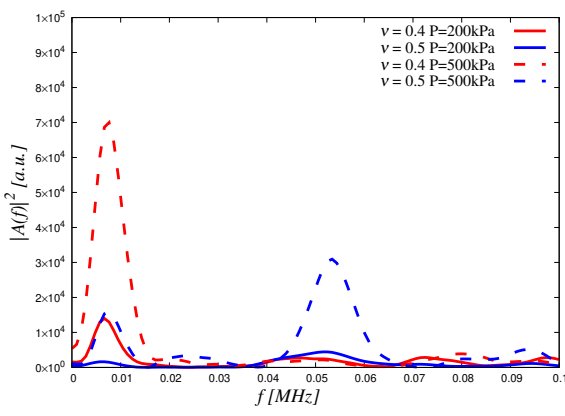

(b)

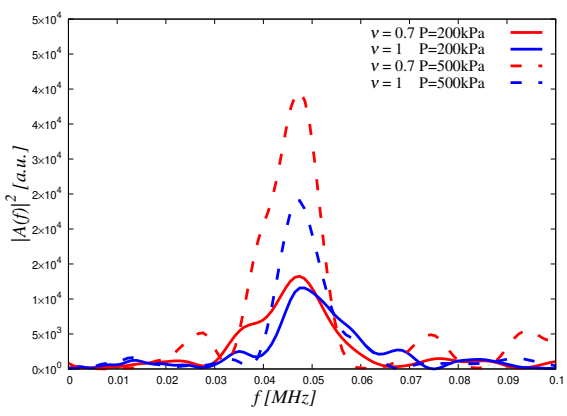

(c)

Figure 4.10: a) Energy against frequency for sample made with $v=0$ (black), 0.1 (red), 0.2 (blue), 0.3 (green) rubber content, at confining pressure of $\mathrm{P}=200 \mathrm{kPa}$ (solid line) and $500 \mathrm{kPa}$ (dashed line); b) Energy against frequency for sample made with $v=0.4$ (red), 0.5 (blue), and 0.6 (green) rubber content, at confining pressure of $\mathrm{P}=200 \mathrm{kPa}$ (solid line) and $500 \mathrm{kPa}$ (dashed line); c) Energy against frequency for sample made with $v=0.7$ (red) and 1 (blue) rubber content, at confining pressure of $\mathrm{P}=200 \mathrm{kPa}$ (solid line) and $500 \mathrm{kPa}$ (dashed line).

\subsection{Damping}

The purpose of this section is to study the loss of energy during the wave propagation with focus on the low rubber content samples, $v \leq 0.3$ rubber. We in- 
troduce a parameter commonly used in geophysics to express the energy loss during wave propagation [185]. The ability of a material to attenuate seismic waves is measured by the dimensionless quantity $Q$ called the quality factor, i.e. attenuation factor:

$$
Q=\frac{\text { Energy of seismic wave }}{\text { Energy dissipated per cycle of wave }}=\frac{2 \pi|A(f)|^{2}}{\Delta|A(f)|^{2}},
$$

where $|A(f)|^{2}$ is the energy of the wave as introduced in Sec. 4.5, and $\Delta|A(f)|^{2}$ is the change in energy between two points in the sample.

Several methods have been developed to compute experimentally the quality factor $Q[31,100,101,107,241,244,256]$. Here we use the Spectral Ratio method based on the assumption that the ratio of the amplitude at two discrete times during the propagation of the wave, varies as a function of frequency [213]. Computation of the spectra of the wavelet and evaluation of the logarithmic ratios for two receivers at depth $H_{1}$ and $H_{2}$ yields to:

$$
\ln \left|\frac{A_{1}(f)}{A_{2}(f)}\right|=-\frac{\pi\left(t_{2}-t_{1}\right)}{Q} f+c
$$

where $A_{1}(f)$ and $A_{2}(f)$ are the amplitude spectra at different length, $f$ is the frequency, $t_{1}$ and $t_{2}$ are the travel time from source to receiver at length $H_{1}$ and $\mathrm{H}_{2}$, and $c$ is a constant which takes into account factors like transmissivity, geometrical spreading, source and receiver response, etc.

Then, the $Q$ factor can be estimated by fitting a straight line to the logarithmic spectral ratio over a finite frequency range. $Q$ is directly related with the slope, $m$, of the best-fit straight line as:

$$
Q=\frac{-\pi\left(t_{2}-t_{1}\right)}{m} .
$$

The above derivation is the basic theory of the classic spectral-ratio method, which is originally derived for the application to VSP (vertical seismic profile) data [245]. It must be mentioned that even when the data is free of noise, the estimated $Q$ can significantly deviate from the true value.

To employ the spectral-ratio method for our mixtures, we perform experiments on samples of two different lengths, $H=70$ and $100 \mathrm{~mm}$. We focus on samples with low rubber content $v \leq 0.3$. Fig. 4.11 shows two signals recorded for samples at the same rubber content and pressure but different heights. For the case of the shorter sample $(70 \mathrm{~mm})$ the wave is shiffted to the left side of 
the time axis. From the time domain, we can calculate the time difference between the two sample peaks $\delta t=t_{2}-t_{1}$. Then, FFT response of two samples are considered limiting the analysis to the part of the signal until the first peak. From Fig. 4.10.a, it is reasonable to assume that over the frequency range of the measurements, $0-0.05[\mathrm{MHz}]$, showing low rubber content samples, $Q$ is a linear function of frequency, as frequencies above this range carry negligible energy (see Fig. 4.11.b). After that, amplitude ratio for the samples with different lengths can be fitted using Eq. 4.14 in order to measure the quality factor $Q$.

Values of damping (loss factor) $Q^{-1}$ are plotted against the rubber content in Fig. 4.12.a for different pressure levels and rubber content $v \leq 0.3$. When the amount of rubber increases, the quality factor parameter $Q^{-1}$ increases in a linear fashion, i.e. the system is more dissipative by increasing the amount of soft inclusions. On the contrary, increasing pressure leads to a decrease of damping as shown in Fig. 4.12.b, where $Q^{-1}$ is plotted versus the confining pressure. The damping of mixtures with higher rubber content is related to the transfer of elastic energy from low to high frequencies.

Combining the observations in Figs. 4.3, 4.7, and 4.12, we summarize that adding roughly $20 \%$ of soft inclusions strongly improves the damping of the system (about 30\%), but also increases its stiffness (up to 15\%) and gives a much lighter sample (about 15\%).

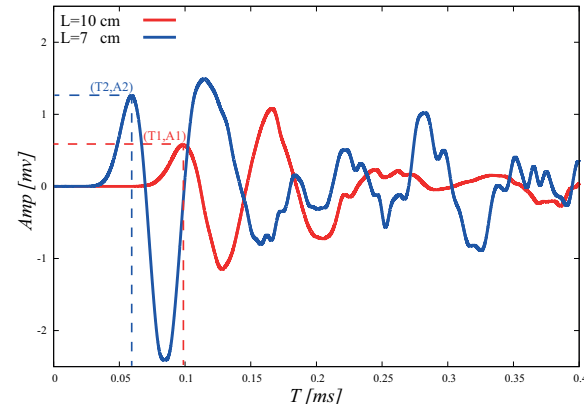

(a)

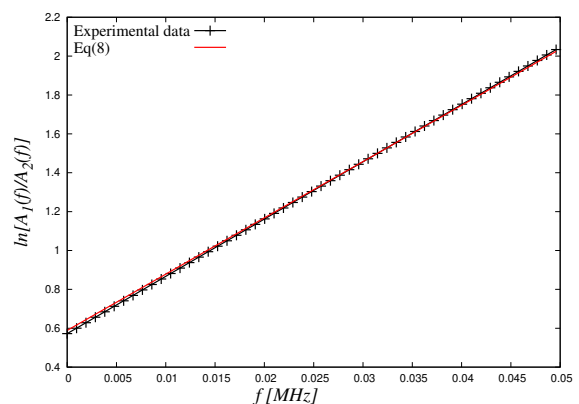

(b)

Figure 4.11: a) Signals recorded for samples with two different lengths (7 and $10 \mathrm{~cm}$ ) at the same rubber content $(v=0.2)$ and the same pressure $(P=500 \mathrm{kPa})$. Peak amplitude of the signal for the short sample is $A_{2}$ and long sample is $A_{1}$, and are marked by ' $\square$ ' and ' $\bullet$ ', respectively. b) Logarithmic amplitude ratio of two given signals in frequency domain fitted by Eq. 4.13. 


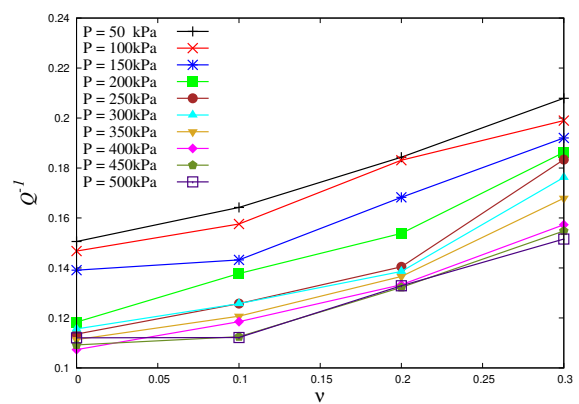

(a)

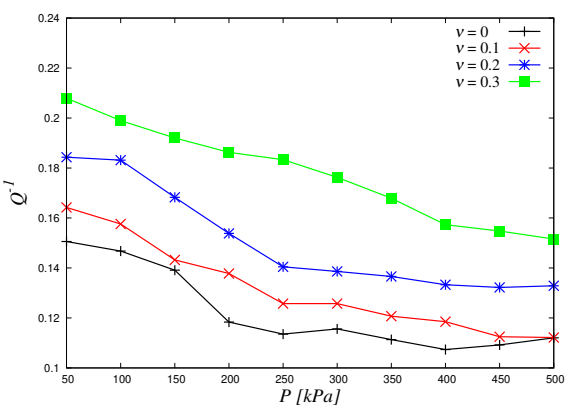

(b)

Figure 4.12: Attenuation factor $Q^{-1}$ for four rubber contents at different pressure level.

\subsection{Conclusion}

In this research, wave propagation experiments were performed to examine the behavior of various idealized mixture compositions $\left(v=\phi_{\text {rubber }} / \phi_{\text {total }}\right.$, with volume fraction $\phi$ ) of monodisperse glass and rubber spheres. Signals inferred from wave propagation tests using transducers were used to infer both bulk stiffness and damping ratio of the mixtures. The experimental data indicate that the glass skeleton controls the behavior for $v \leq 0.3$, while the rubber skeleton prevails at $v \geq 0.7$. There is a considerable drop in the modulus $M$, only at intermediate mixtures $(0.3<v<0.6)$ where the transition from stiff to soft regime occurs. Interestingly, we found that waves propagate faster in $v \approx 0.2$ than in a purely glass sample $v=0$ at all stress levels, which might be of interest for many industrial applications to optimize processes and bulk materials.

Furthermore, we studied the frequency response of signals using a Fast Fourier Transform analysis at different pressure levels to access the frequency content of the arriving signal. Increasing confining pressure was not seen to cause any change in the dominant frequency, while the majority of the elastic energy moves from low to high frequency when the rubber content increases in a small range from $v=0.4$ to $v=0.5$, with a new sharp peak appearing. Comparing the peak frequencies of samples, the transition from stiff to soft dominated media was clearly observed.

Finally, the damping in samples with low rubber content was determined introducing of spectral-ratio approach. As expected, a systematical increase of damping with increasing rubber content was attained, while increasing pressure 
reduces damping.

The relationship between the P-wave modulus and the attenuation factor at low rubber content samples reveals an interesting phenomenon: although the stiffness remains almost unchanged with increasing rubber volume (or even increases slightly), the damping increases continuously. Especially, an optimal mixture was attained for rubber content close to $v=0.2$ at high pressure, that shows the highest P-wave modulus and significant damping.

Future work includes the measurements of S-waves in glass-rubber mixtures and numerical simulations (using DEM and FEM) to reproduce the behavior of glass-rubber mixtures tested experimentally to gain more micro-mechanical insights at the microscale.

\section{Acknowledgment}

Financial support for the study was provided by grant FP7 (ITN 607453) from the European-Union Marie Curie Initial Training Network, T-MAPPP, see http://www.t-mappp.eu/ for more information.

\section{Appendix. A}

Several difficulties such as the selection of travel distance, the determination of travel time, and near filed effects affect the measurement of P-wave velocity. These issues have been addressed in Refs. [136, 172, 173, 183, 184]. Once these boundary and scale effects are evaluated and their effects are considered, the travel time between source and receiver bender elements can be determined [9]. The recorded traces provide a mean to measure the P-wave travel time, calculation of the P-wave velocity, and evaluate the corresponding compression modulus (if the density is known). Concerning the travel time and distance, necessary to calculate the wave velocity, the determination of travel distance (distance between transducers) is generally considered less problematic of the two. The determination of the travel time, on the other hand, is more controversial.

In Fig. 4.4, some characteristic features of the transmitted and received signal were marked. Feature A marks the impulse of the transmitted signal, and on the received signal the characteristic features are first-arrival $\mathrm{B}$, first-local maximum C, and first-local minimum D. Generally, the signal transmitted till the D-point is called the first event which carries the essential information. The 
first arrival method calculates the time difference between the first peak in the transmitted signal (A) and the first deflection observed in the output signal (B). But, the peak-peak method takes the time between the first peak (A) of the input signal and the first and/or second peak ( $C$ and/or $D)$ of the received signal. Here, we pick consistent peak-peak travel time (difference between time of A and C), since selecting the first arrival pin is sometimes less straightforward [184].

The P-wave modulus obtained by different travel time selection criteria are shown in Fig. 4.13 for samples confined at $200 \mathrm{kPa}$ pressure. As it was expected from Fig. 4.13, the results obtained using the first arrival point (B in Fig. 4.5) give the lowest value than the other two. It is noteworthy to mention that the qualitative trend of the P-wave modulus is similar for the three methods, as illustrated in Fig. 4.13.

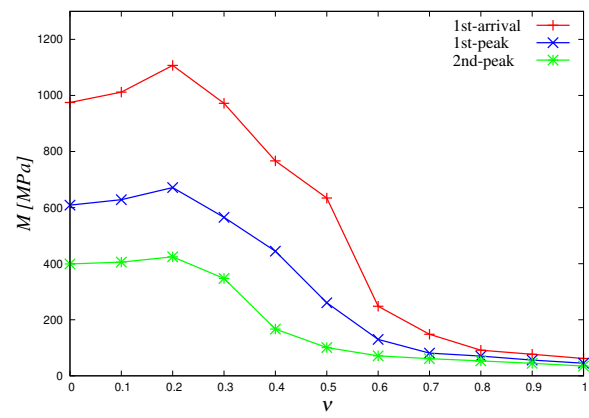

Figure 4.13: P-wave modulus versus fraction of rubber obtained by the first deflection, first peak and second peak points.

\section{Appendix. B}

Complementary to the FFT analysis results, the energy is plotted for a sample with rubber content of $v=0$ at different confining pressures (from $P=50$ to $500 \mathrm{kPa}$ ) in Fig. 4.14. This observation prevails the fact that the main frequency of glass media remains unchanged by increasing the confining pressure. Moreover, one can determine the main frequency of glass dominated samples from this figure. 


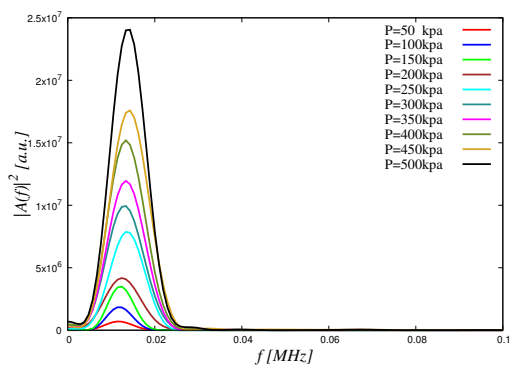

Figure 4.14: Energy against frequency for a sample made with glass particles, $v=0$, at different confining pressure. 


\section{Chapter 5}

\section{Stress based multi-contact model for discrete-element simulations}

The Beginning is lost; the End stretches into eternity. Don't bother with them, they're all irrelevant.

And since all is really nothing, then nothing is truly everything.

Farid ud-Din Attar

The aim of this study is to introduce a stress-based non-binary contact model missing in classical discrete element method (DEM). To tackle this issue, the classical Hertzian force-displacement law is generalized by utilizing the trace of the particle stress tensor to make contacts dependent on all other contacts of a particle. Therefore, the stress tensor commonly used for post-processing reasons (i.e. in coarse-graining methods), it is used to account for multiple contacts simultaneously acting, on a single particle. Simulation results for uniaxial confined compression employing our new multi-contact model were compared with the classical discrete element formulation, an existing strain-based multi-contact model, and experimental data. The satisfactory agreement between these results supports the validity of our new contact model. Futhermore, test examples at higher load levels show that our new contact model formulation is able to capture also the stronger non-linearity at higher stresses. Due to its simplicity, the proposed multi-contact 
model can easily be integrated in any DEM implementation, remaining relatively fast when compared to more complex methods or even a discretized method e.g. by FEM of single particles. ${ }^{1}$

\subsection{Introduction}

Granular media, such as sand, are ensembles of dissipative, athermal grains that interact through repulsive and frictional contact forces. An interesting feature of granular materials is the fact that they can behave as solids or liquids, and show peculiar mechanical properties like dilatancy, history dependence, ratcheting and anisotropy [37, 148]. Despite their simplicity and omnipresence, the physics of granular materials is still not fully understood and this leaves many open questions in different fields, e.g. physics, process engineering, material science, geotechnical engineering, etc.

The discrete element method (DEM), pioneered by Cundall and Strack [42, 150, 235], models granular materials numerically as a collection of particles rather than as a continuum, and the bulk behaviour of granular materials depends on the collective interactions among individual particles. Each discrete element has its own individual movement that can be traced by explicitly integrating the governing differential equation based on the Newton's second law of motion.

DEM has been used in a wide variety of applications such as powders [162], ceramics [214], granular flows [36], pharmaceutical and food industries [25, 197]. Despite the fact that DEM is a very efficient tool to study these applications, modeling of confined high to extreme compression with DEM is still a challenge. In classical DEM, the so-called soft particle approach $[151,236]$, particle deformations mimicked by overlaps between contacting particles. When an overlap is detected, the contact forces between two particles are calculated by a contact law. The general assumption made is that contacts between particles are independent and therefore, contact forces are resolved locally. This assumption is only true in cases when particles deformation is small. For cases with large deformations classical DEM is limited on capturing the required deformation $[82,190]$.

One way to introduce deformability in a particle model is by applying the Multiple Particle Finite Element Method (MPFEM) [67, 82, 196] where each

\footnotetext{
${ }^{1}$ Giannis, K., Taghizadeh, K., Schilde, C., Finke, J.H., Celigueta, M.A., Luding, S., \& Kwade, A. Granular Matter, submitted Jul. 2019.
} 
individual particle is being meshed with finite elements. Another way is to combine FEM and DEM [59, 231], a method that embodies contact detection algorithms of DEM in the framework of FEM. Particle deformability can be also modeled with other continuum based dicretization methods such as the material point method (MPM) [89, 178] or the bonded particle method (BPM) [48]. The main advantage of these methods is their ability to deal with anisotropic deformations of single particles. Therefore, these models are able to model arbitrary shapes after deformation. However, the main challenge is their high computational cost that hinders their use for cases with a large number of particles.

Following a different strategy, there have been attempts to introduce deformable particles in the framework of DEM. A simple approach was proposed by Haustein et al. [83], by expanding the radius of the spherical particles. The particle deformation from its overlapping area is redistributed on the free surface of the particle, such that the volume of the particle is kept constant. A more sophisticated model was presented by Kloss et al. [110]: Based on the single particle average stress tensor each particle is allowed to isotropically deform by decreasing its particle radius and increasing its internal density. In addition, Rojek et al. [202] proposed the so-called deformable discrete element method (DDEM). In this approach, particles are uniformly deformed under uniform internal stress, which generates a uniform strain inside the particle; and contact forces are evaluated using the local and newly formed global overlap caused by the deformation of the particle.

The need of having deformable particles in the framework of DEM has led to the formulation of the multi-contact discrete element method (MC-DEM). Brodu et al. incorporated explicitly the mutual influence of contacts acting simultaneously on a single particle [22]. In this method, the overall deformation of a particle is evaluated in terms of the strain field induced by other contacts acting on the particle. Brodu et al. compared the results of conventional DEM, their new model, and experimental results for uniaxial compression of hydrogel particle packings in order to show the ability of their model to reproduce the real physical data [22]. Furthermore, the evolution of the microstructure, using the multi-contact approach, was compared against the non-local contact formulation presented by Gonzalez et al. [75, 76].

Another idea is to employ the stress instead of the strain field in calculations. For this reason, a multi-body contact law that accounts for contact dependency was presented by Frenning [60]. In this case, particle deformation is approximated by truncated spheres; and the contact force was expressed as the product of the sum of normal stresses (independent of contacts direction) and a con- 
tact area (that differs between the pair of contacts). The contact dependency among contacts acting on the same particle was also considered in the work of Celiqueta [26]. However, in this case the contact dependency was restricted to contacts acting perpendicular to the contact direction considered.

This study proposes a new formulation in the context of multi-contact discrete element method based on the stresses acting on single particles to account for multiple contacts acting on a particle. The overall deformation of the particle contact has been induced by other contacts acting on the particle. The main difference between the new methodology and the one introduced by Brodu et al. [22] is that this model is based on the stress applied on the particle not strain.

This paper is organized as follows: In the second section, the classical discrete element method is presented. A general overview on the exiting multicontact model proposed by Brodu et al. [22] is given in section three. Furthermore, the formulation and implementation of our new multi-contact model is presented. At the end of section three, test cases with collisions of three and five particle collisions are considered to show how the multi-contact models are performing in comparison to classical DEM. In section four a case a single compressed rubber sphere presented followed by section five where a case of uni-axial compaction using hydrogel balls is presented. Additionally, a numerical test on a harder material (rubber spheres) is given and a case of uni-axial compaction using stiff material, glass beads, where successfully tested. Finally, the computational cost of the performance of the multi-contact models is discussed in section 6 . We conclude our work in the section five and finish with some outlooks.

\subsection{Discrete Element Method}

The approach towards the microscopic understanding of macroscopic particulate material behaviour is the modelling of particles using the so-called discrete element method (DEM), a numerical scheme originally formulated and developed by Cundall et al. [42].

DEM is a straightforward implementation to solve the transitional and rotational equations of motion for a system of many interacting particles:

$$
m_{i} \overrightarrow{\ddot{a}}_{i}=\vec{f}_{i}+m_{i} \vec{g} \quad I_{i} \overrightarrow{\dot{\omega}}_{i}=\vec{\tau}_{i}
$$


where $m_{i}$ is the mass of the $i$-th particle with position $x_{i}$. It is subjected to two kinds of forces, one due to contacts with other particles $\left(\vec{f}_{i}=\sum_{c} \vec{f}_{i}^{c}\right)$ and one due to volume forces (i.e. gravity acceleration, $\vec{g}$ which are neglected in this study. $I_{i}$ is the spherical particles moment of inertia, $\dot{\vec{\omega}}_{i}$ is the angular velocity and $\vec{\tau}_{i}=\sum_{c}\left(\vec{l}_{i}^{c} \times \vec{f}_{i}^{c}+\vec{q}_{i}^{r}+\vec{q}_{i}^{t}\right)$ is the total torque, where $\vec{l}_{i}^{c}$ is the branch vector and $\vec{q}_{i}^{r}$, $\vec{q}_{i}^{t}$ are torques due to rolling and torsion.

The basis of DEM are force laws that relate the interaction force to the overlap of two particles. The force can be decomposed into a normal and tangential component $\vec{f}_{i}^{c}=\vec{f}_{n}+\vec{f}_{t}$. With the normal and tangential forces acting on all particles, one can numerically integrate the equations of motion and obtain the next position of particles. Below, we will describe a force law used in this research.

\subsubsection{Normal contact law}

The elementary units of granular material are mesoscopic grains which deform under contact forces, induced by an externally applied stress. For realistic modelling of the deformation of particles we relate the interaction force to the overlap $\delta$ of two particles. Note that the evaluation of the inter-particle forces based on the overlap may not be sufficient to account for the inhomogeneous stress distribution inside the particles. Two particles only interact if they are in contact, resulting in an overlap:

$$
\delta=\left(r_{i}+r_{j}\right)-\left(\vec{x}_{i}-\vec{x}_{j}\right) \cdot \vec{n}>0,
$$

where $\vec{n}=\frac{\vec{x}_{i}-\vec{x}_{j}}{\left|\vec{x}_{i}-\vec{x}_{j}\right|}$ is the unit vector pointing from particle $j$ to particle $i$.

From the motion equations, Eq. 5.1, it is apparent that the contact force is needed to determine particles trajectory. This force is calculated through a contact force law, which is a simplification of the contact between two particles. Hertzian contact model is the most common used contact model in DEM which is a non-linear model based on the Hertz theory of elastic contact [98, 150, $234,238,270]$. This model assumes that the particles are spherical and do not deform during the simulation. In addition, this model considers binary contacts between two particles which means particles are in contact through a single point during their collisions.

$$
f^{n}=f_{e l}^{n}+f_{v i s c}^{n}=\frac{4}{3} E^{*} \sqrt{r_{e f f} \delta^{n}} \delta^{n}+\eta_{n} \dot{\delta^{n}}
$$


with $r_{e f f}=\frac{r_{i} r_{j}}{r_{i}+r_{j}}$ as the effective radius and $E^{*}$ is the effective Young's modulus, $\frac{1}{E^{*}}=\frac{1-v_{i}}{2 G_{i}}+\frac{1-v_{j}}{2 G_{j}}$. In this expression, $v$ and $G$ represent the particles Poisson's ratio and shear modulus, respectively. In reality, particles collisions are inelastic, i.e. energy loss occurs during collisions. Here, the dissipation is related to relative velocity $\left(v_{n}^{r e l}=-\left(\vec{v}_{i}-\vec{v}_{j}\right) \cdot \vec{n}=\dot{\delta}^{n}\right)$ of interacting particles with the viscoelastic damping constant for normal contact viscosity $\eta_{n}$ which is an intrinsic material parameter.

\subsubsection{Tangential force law}

For the tangential degrees of freedom, we only consider sliding resistance between particles, rolling and torsion resistance are neglected for the sake of simplicity. Tangential forces are linked to friction between two particles generated by the relative motion of the two particles. This is a source of energy dissipation. The sliding/sticking friction model is based upon Coulomb's friction law. Two friction models are used, static $f^{t} \leq \mu_{s} f^{n}$ and dynamic friction, $f^{t}=\mu_{d} f^{n}$; where $\left(\mu_{s}\right)$ is the static and $\left(\mu_{d}\right)$ the dynamic friction coefficient.

Static friction occurs when two particles do not involve micro-slip at the contact surface. In the case of static friction, the friction force (tangential force) $f^{t}$ that is exerted between the surfaces of two particles where the particles having no relative motion can not exceed the value given by Coulomb's law using static friction coefficient (i.e. $f^{t} \leq \mu_{s} f^{n}$ ). For the static case, a restoring force is needed to compensate for the non-zero tangential force from the Coulomb's law. To determine if the particle experiences static or dynamic friction, a tangential test-force is calculated. The tangential test-force is calculated in the same form as the normal force using the Mindlin tangential law:

$$
f^{t}=f_{e l}^{t}+f_{v i s c}^{t}=8 G^{*} \sqrt{r_{e f f} \delta^{n}} \delta^{t}+\eta_{t} \dot{\delta}^{t}
$$

where $\delta^{t}$ is the relative shear displacement between the two particle centers, and $G^{*}$ is the effective shear modulus, $\frac{1}{G^{*}}=\frac{2-v_{i}}{G_{i}}+\frac{2-v_{j}}{G_{j}}$. Likewise the normal direction, we consider a dissipation term along the tangential direction using of viscosity term $\eta_{t}$ [239].

Dynamic friction happens when the tangential component of force $f^{t}$ is exceeding the maximum value of static force $\left(\mu_{s} f^{n}\right)$, hence two particles start to slide against each other. 


\subsection{Deformable particle models}

In the classical formulation of DEM, each contact force is considered to be a local phenomenon and it is resolved locally independent of the effect of other contact forces in its vicinity. However, in reality every contact between particles is affected by neighboring particles acting simultaneously. Deformation of particles induces non-linearity at the same and all other contacts, and can cause the formation of new contacts which is ignored in the conventional DEM approach. The essential ingredient is to consider an overall grain shape deformation induced by particle contacts.

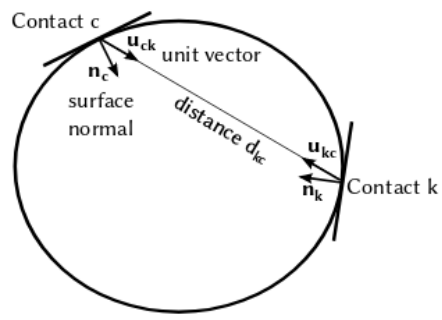

Figure 5.1: Influence of one contact onto another. Contacts are not restricted to the surface of a sphere, their position is consistent with the grain deformations [22].

Here, we adopt the nonlocal contact formulations that account for the interplay of deformations due to multiple contact forces acting on each single particles. Such nonlocal formulations remove the classical assumption that contacts acting on a single particle is formulated locally as independent pair interactions.

In the following, we first explain a strain-based approach proposed by Brodu et al. [22] and after that, we propose a new method, based on the stress and thus the elasticity of materials. Finally, we compare the explained models with the classical DEM results of simple test cases.

\subsubsection{Multi-contact strain based model}

To overcome the above summarized challenge, Brodu et al. [22] proposed a multi-contact approach to improve the predictive power of DEM methods, while 
retaining their conceptual simplicity. In this approach, the mutual influence of contacts is modeled by using information on deformations induced by one contact force on the other contacts acting on the grain. The displacement fields imposed by neighboring contacts in normal direction, $\delta_{k \rightarrow c}$ are added to the particle deformation at the local contact, $\delta_{c}$. After that, force calculation can be computed based on the added displacement fields $f_{H} \propto\left(\delta_{c}+\sum_{k} \delta_{k \rightarrow c}\right)^{3 / 2}$ with the displacement fields $\delta_{k \rightarrow c}$ equal to:

$$
\begin{aligned}
\delta_{k \rightarrow c}=-\gamma & \frac{(1+v) f_{k}^{c}}{2 \pi E d_{k c}}\left[\left(\vec{n}_{k} \cdot \vec{u}_{k c}\right)\left(\vec{n}_{c} \cdot \vec{u}_{k c}\right)+\right. \\
& \left.(3-4 v) \vec{n}_{k} \cdot \vec{n}_{c}-(1-2 v) \frac{\left(\vec{n}_{k}+\vec{u}_{k c}\right) \cdot \vec{n}_{c}}{1+\vec{n}_{k} \cdot \vec{u}_{k c}}\right]
\end{aligned}
$$

where $f_{k}^{c}$ is the force at contact $k, E$ the Young's modulus of the material, and $v$ its Poisson's ratio. $\gamma$ is an adjustable prefactor which accounts empirically for the geometry. For non-compressible materials (low Poisson's ratio) the prefactor is set to be $\gamma=0.5$ and for compressible particles $\gamma=1 . d_{k c}, \vec{n}$, and $\vec{u}_{k c}$ are the distance between contact points, normal vectors of contact surfaces, and unit vectors between contacts: These parameters are depicted in Fig. 5.1. Since, the main feature is the strain field around particles, hereinafter the multi-contact model proposed from Brodu et al. [22] will be called MC-strain. Normal contact forces are calculated through this equation:

$$
f_{e l}^{n}=\frac{4}{3} E^{*} \sqrt{r_{e f f}}\left(\delta^{n}+\sum_{k} \delta_{k \rightarrow c}\right)^{\frac{3}{2}}
$$

\subsubsection{Multi-contact stress based model}

In order to overcome the inherent assumption of the classical DEM which treats each contact locally as a binary pair-interaction, we propose a non-local model to describe a new space in which the mutual influence of contacts is taken into account. To do this, we took the advantage of the trace of the particle stress tensor and its information.

Typically, the particle stress tensor was used to access the stresses over a part which by definition computes the stress level exerted on a particle by its neighboring particles after averaging the sum of all contact stresses within its volume 
$[56,127,151,259,271]:$

$$
\sigma^{p}=\frac{1}{V^{p}} \sum_{c=1}^{C^{P}} l_{\alpha}^{c} \otimes f_{\beta}^{c}
$$

The stress tensor incorporates a multiple contacts interaction exerted on the particle. With that, we collect information from all local contacts around the particle. Using this information, we can generalize the typical DEM normal force law in a way that the trace of the stress tensor is used for on-line calculations. In this concept, the new contact forces on each pair are depending also on the trace of the stress tensor of neighbouring particles, hence, an anisotropic deformation of a particle can be accounted for. Fig. 5.2 depicts a schematic concept of the new approach. In the following, our model is called MC-stress since our formulation starts with the stress tensor acting on a single particle. As it can be seen from the figure, an additional term is added into the conventional DEM $\left(P_{i j}\right)$. This term consists of the trace of stresses applied on the primary ( $i$-th) particle $\left(\operatorname{tr} \sigma_{i}\right)$, and the trace of stresses applied on the secondary ( $j-$ th) particle $\left(\operatorname{tr} \sigma_{j}\right)$.

Convectional DEM
$\rightarrow$ Binary contacts
$\mathrm{F}_{\mathrm{i} \rightarrow \mathrm{j}}=\mathrm{f}(\delta)$
Alternative model
$\rightarrow$ Multi-contact stress based
$\mathrm{F}_{\mathrm{i} \rightarrow \mathrm{j}}=\mathrm{f}\left(\delta, \mathrm{P}_{\mathrm{ij}}\right)$
$\mathrm{P}_{\mathrm{ij}}=\left(\operatorname{tr}\left(\sigma_{\mathrm{i}}\right)+\operatorname{tr}\left(\sigma_{\mathrm{j}}\right)\right) / 3$

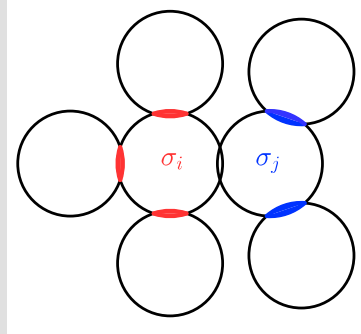

Figure 5.2: For any pair-interaction, a term dependent on the average stress between particle $i$ (red) and particle $j$ (blue) is added to the normal force law.

A new term can be added into the former normal force calculation by including the trace of stress tensor:

$$
f_{e l}^{n}=f_{H}+(\beta v A) P_{i j}
$$


The first term in Eq. 5.8 was defined earlier (Eq. 5.3). The second term carries the information from neighboring particles acting on the particle. In this expression, $\beta$ is an adjustable prefactor that empirically accounts for the hardness of each particle, $v$ is the Poisson's ratio, and $A$ is the contact area at interface of the active pair of contacts. The isotropic component of the stress is the pressure $P_{i j}=\frac{1}{3}\left(\operatorname{tr}\left(\sigma_{i}\right)+\operatorname{tr}\left(\sigma_{j}\right)\right)$ and where $\operatorname{tr}(\sigma)=\left(\sigma_{x x}+\sigma_{y y}+\sigma_{z z}\right)$

At this point and for the multi-contact models that were used here it is necessary to highlight the similarities and differences. MC-strain model estimates the particle deformation by using analytical formulas given in elasticity theory which is a similar assumption in case of MC-stress model. In both models, the overall deformation of a particle is calculated, and the deformation is taken into account for calculation of new contact force. The MC-strain model takes into account a pseudo geometric deformation, the contact forces that calculated at a previous time-step by equation Eq. 5.6 are now used to generate a new overlap based on Eq. 5.5, i.e an explicit calculation. Then this new overlap inserted into Eq. 5.6 yields to the new contact forces. On the contrary, with the MC-stress the required deformation is captured by gradually increasing the stress level experienced by the particle. The contact forces at the current time-step are used to compute the trace of the stress tensor between interacting particles, i.e an implicit calculation. Based on Eq. 5.8, new contact forces are calculated for every pair of interactions.

The new multi-contact model, MC-stress, was implemented in the LIGGGHTS-DEM platform [109]. LIGGGHTS in its philosophy takes the benefit of Newton's third law, where forces are computed for each pair of particles once [193] in order to have an optimized and fast algorithm. By exploiting this, we can integrate our new formula without violating the momentum balance $\left[\vec{f}_{i j}^{c}=-\vec{f}_{i j}^{c}\right]$. In the appendix 5.7 the pseudo-code of the algorithm used is briefly described.

\subsubsection{Modeling test cases}

To see the differences between given models and the classical DEM, we create a set of simple reference cases that includes a system of three and five particles. On the other hand, comparing results between the classical DEM, MC-strain, and MC-stress validates the implementation of MC-stress. Since the behavior of hydrogel grains was thoroughly examined in Refs. [22, 23], and it is the first material studied under the framework of multi-contact modeling, its material properties were used in this study. Moreover, we have used a harder material 
(rubber) to further investigate the models. Material parameters used for the simulations are shown in table 5.1.

\begin{tabular}{llll}
\hline Material properties & Hydrogel & Rubber & Glass \\
\hline Density $\left(\frac{\mathrm{kg}}{\mathrm{m}^{3}}\right)$ & 11.5 & 2000 & 2500 \\
\hline Diameter* $(\mathrm{cm})$ & 2 & 2 & 0.4 \\
\hline Young's modulus $(\mathrm{Pa})$ & $23.3 \cdot 10^{3}$ & $1.85 \cdot 10^{6}$ & $65 \cdot 10^{9}$ \\
\hline Poisson's ratio $(-)$ & 0.5 & 0.46 & 0.24 \\
\hline Coefficient of restitution $(-)$ & 0.95 & 0.7 & 0.98 \\
\hline Friction coefficient $(-)$ & 0.03 & 0.5 & 0.55 \\
\hline
\end{tabular}

Table 5.1: Input parameters used for simulations from [22, 76, 83].

It is clear that multi-contact models do not show any difference with the classical DEM in case of two sphere interactions. Therefore, the first reference case consists of a central sphere with a diameter of $2 \mathrm{~cm}$ that has been placed between two spheres with identical characteristics (see Fig. 5.3.a). Initial velocities of $10 \frac{\mathrm{m}}{\mathrm{s}}$ were applied in opposite directions such that the central sphere gets compressed, i.e. its overlaps with two neighbouring particles increase. In addition, we study a more complex case by adding two more spheres added along the $y$-axis, as shown in Fig. 5.3.b, in order to better resemble a typical confining situation. By studying these cases we can meticulously examine how deformation develops under different contact laws.

\section{Test cases using hydrogel}

The systems examined in the following contain $N=3$ particles and $N=5$ particles with radii $r_{i}=1 \mathrm{~cm}$. The typical contact duration is $t_{c}=2.87\left(\frac{\left(m^{*}\right)^{2}}{r v\left(E^{*}\right)^{2}}\right)^{\frac{1}{5}}$ [84] which leads to $t_{c}=0.0018 \mathrm{~s}$, and accordingly an integration time-step of $\delta t=0.00001 \mathrm{~s}$ ( $t_{c} \gg \delta t$, in order to allow for "safe" integration of the equations of motion) are chosen for simulations. Fig. 5.4 shows the kinetic energy of systems simulated by three and five hydrogel particles. Overlap of central particle in case of three and five particles simulation are given in Fig. 5.5.

Looking at Fig. 5.4, we can see a faster reduction of the energy with the multi-contact implementations followed by an increase in the energy after the 

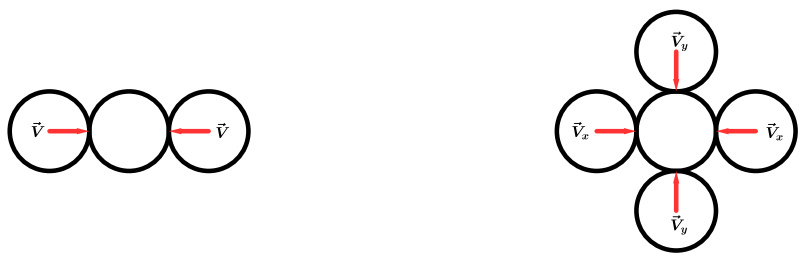

(a)

(b)

Figure 5.3: Schematic representation of (a) three and (b) five particles interaction.

maximum indentation is reached (zero velocity). The shift of energy is related to the fact that the multi-contact models reach the maximum overlap earlier than the conventional model. The total forces acting on a single particle are gradually higher using multi-contact models. These forces are then used and the DEM equations of velocity and position for each individual particle are integrated in time using an explicit scheme.

LIGGGHTs uses the Velocity-Verlet integration scheme [63, 164] to perform time integration, the extended equations can be found in the appendix 19 - Briefly the equations for the velocity and position of each particle are: $\mathrm{v}_{i}^{\text {new }}=( \pm) \mathrm{v}_{i}^{\text {old }}+( \pm) \frac{\delta t}{2 m} f_{i}^{\text {old }}$ and $x_{i}^{\text {new }}=( \pm) x_{i}^{\text {old }}+( \pm) \delta t \mathrm{v}_{i}^{\text {new }}$, with signs are changing according of particle's direction.

It is clear that for higher total forces ( $\delta t, m=$ constant) we have lower magnitudes of the velocity and and as a consequence less change in a particle's position. With that we can say that for multi-contact models we have lower velocities when we compress the particles due to higher resistance and higher velocities at the decompression due to higher repulsive forces.

Overlap figures reveal that the contact collision is shorter while using multicontact models since more resistance is applied against further compression. The maximum overlap of the MC-strain contact model is the highest in comparison to the two others due to the generation of new overlaps at each time step, i.e. in MC-strain model a "correction" term of the overlap is added. Contradictorily, the MC-stress model resists against compression (and decompression) 
which leads to a lower value of maximum overlap in comparison to other cases.

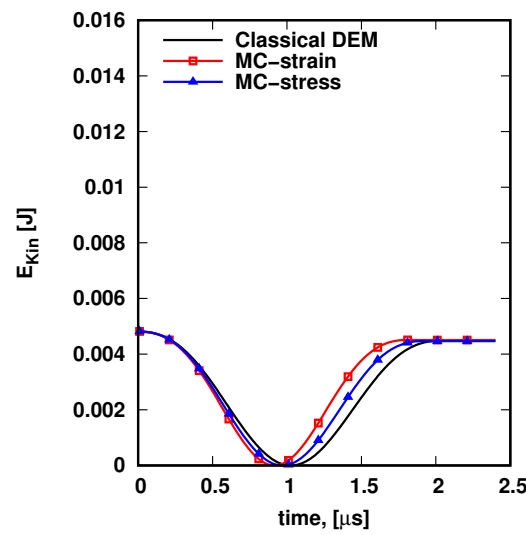

(a)

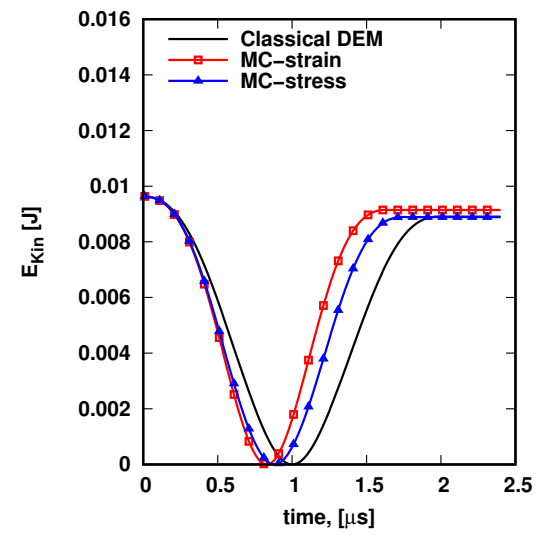

(b)

Figure 5.4: Kinetic energy of test cases of (a) three and (b) five hydrogel spheres using different contact models as indicated in the inset.

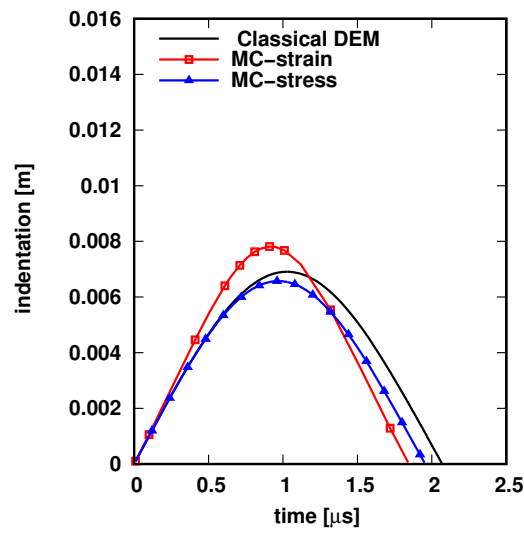

(a)

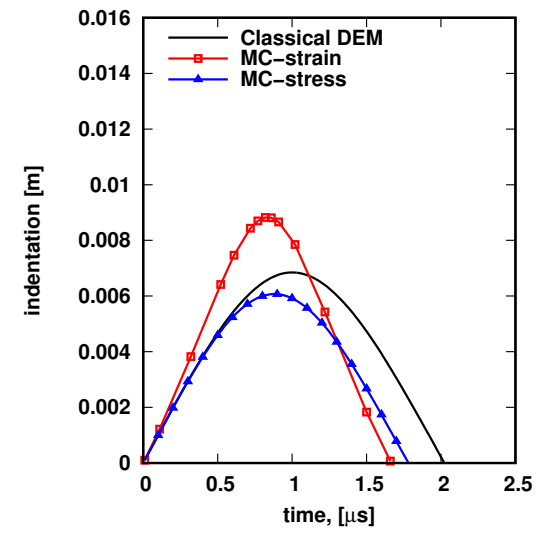

(b)

Figure 5.5: Overlap of central particle for the test cases of (a) three and (b) five hydrogel spheres using different contact models as indicated in the inset. 


\section{Test cases using rubber}

In addition to hydrogel tests, we examine the three and five particles tests using a harder material (rubber) to confirm that the earlier observations remain independent of the particles properties. The contact duration of $t_{c}=0.0025 \mathrm{~s}$ and an integration time-step of $\delta t=0.00002 \mathrm{~s}$ are considered in simulations using rubber particles. Likewise the hydrogel tests, we can see that the kinetic energy plots show the same trend with stronger dissipation of energy while multi-contact models are used (Fig. 5.6). Interestingly when our system gets more confined the reduction of the kinetic energy is the same for both multi-contact models (Fig. 5.6.b) and the increase of the kinetic energy is higher due to the higher repulsive forces acting on a particle by applying MC-strain model. As it is observed in Fig. 5.7, the maximum overlap at the peak is reached by the MC-strain model, and the minimum is given by the MC-stress. It is not surprising since the basis of MC-strain is on adding new overlaps, and MC-stress is on correcting the force by adding new forces, i.e. stiffening of particles.

The results of test cases using hydrogel and rubber properties evidently show that the performance of multi-contact models is strongly dependent upon the confinement level of a system. However, it is hard to say which model shows a more robust agreement without comparing them to experimental data. Therefore, in the next sections, we will compare different models for real tests of uni-axial compression.

\subsection{Uniaxial unconfined compression of a single rubber sphere}

To validate the performance of the mutli-contact formulations we here consider a uniaxial compression of one elastic rubber sphere of radius $1.0 \mathrm{~cm}$ and elastic properties that are shown in table 5.1 and compare the simulated results with experimental results from Tatara [237]. The sphere was pressed between two rigid plates as it shown in Fig. 5.8 to a deformation up to $0.4 \mathrm{~cm}(40 \%$ of the undeformed sphere). In Fig. 5.9 the results of the validation are presented. As we can see Hertz theory is applicable only for small deformations with an upper limit of $\frac{\delta}{R}<0.1$ ( $10 \%$ of the undeformed sphere). In sharp contrast the multicontact models are show a very good agreement with the experimental data for small up to large deformations. 


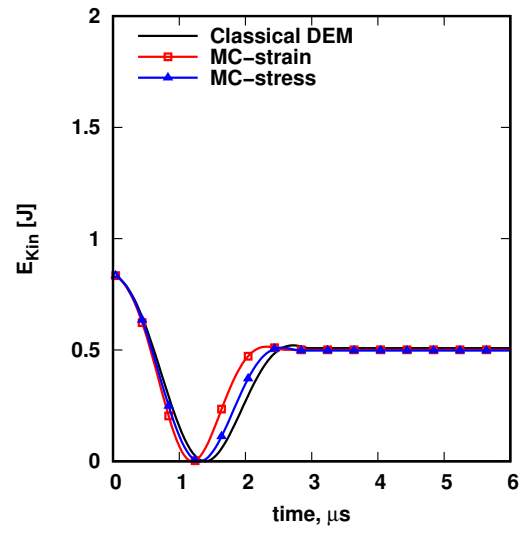

(a)

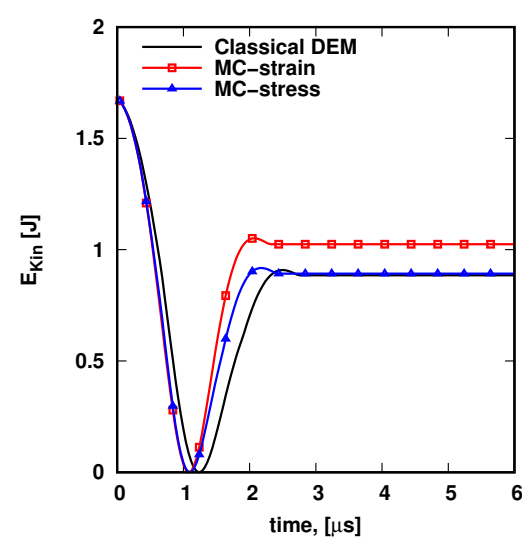

(b)

Figure 5.6: Kinetic energy of test cases of (a) three and (b) five rubber spheres using different contact models as indicated in the inset.

\subsection{Uniaxial confined compression}

\subsubsection{Compression using hydrogel balls}

After the validation of the new approach in the previous section, here, we simulate a more complex system which contains many particles and applying different contact models. The objective of this section is to encounter how our model is performing in comparison to existing models (classical DEM and MC-strain) and available experimental data given in Ref. [22]. The system considered here is a rectangular box, with dimension of $0.165 \times 0.165 \times 0.167 \mathrm{~m}^{3}$ along $x-y-z$ directions in which 514 polydisperes balls with a mean diameter of $2.1 \mathrm{~cm}$ were placed. The sample was first compressed uni-axially along the $z$-direction to a maximum target strain; after that it was decompressed. In the following, we first consider hydrogel properties (shown in table. 5.1) as the material characteristic of balls in the simulation. Results are compared with the existing experimental data.

Fig. 5.10 shows the results obtained from the compression/decompression simulation of the box filled with hydrogel spheres. It is not surprising to see the failure of the classical DEM in representing the experimental data, especially in case of hydrogel particles since the classical DEM can not treat simulation 


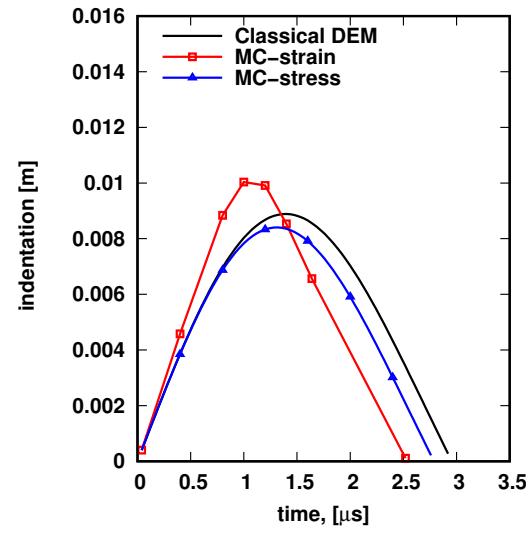

(a)

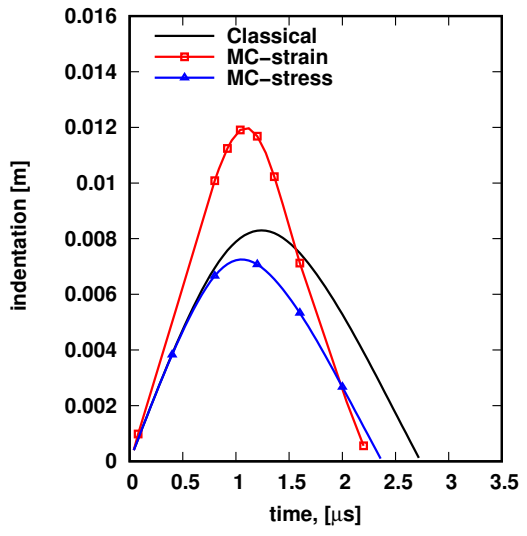

(b)

Figure 5.7: Overlap of central particle for the test cases of (a) three and (b) five rubber spheres using different contact models as indicated in the inset.

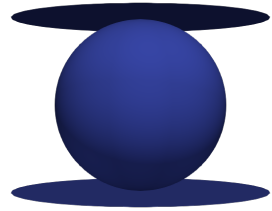

(a)

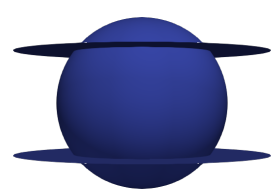

(b)

Figure 5.8: Schematic representation of a rubber sphere uniaxially compressed between two rigid plates.

of soft materials. The MC-stress model with a prefactor of $\beta=1$ offers a fair performance, but not as good as the MC-strain model with a prefactor of $\gamma=1$. Yet, we are far from describing the experimental data with these parameters presented in Fig. 5.10. To obtain a better agreement between simulation and experiment, one can tune the prefactor of $\gamma$ and $\beta$ in both models. Note that 


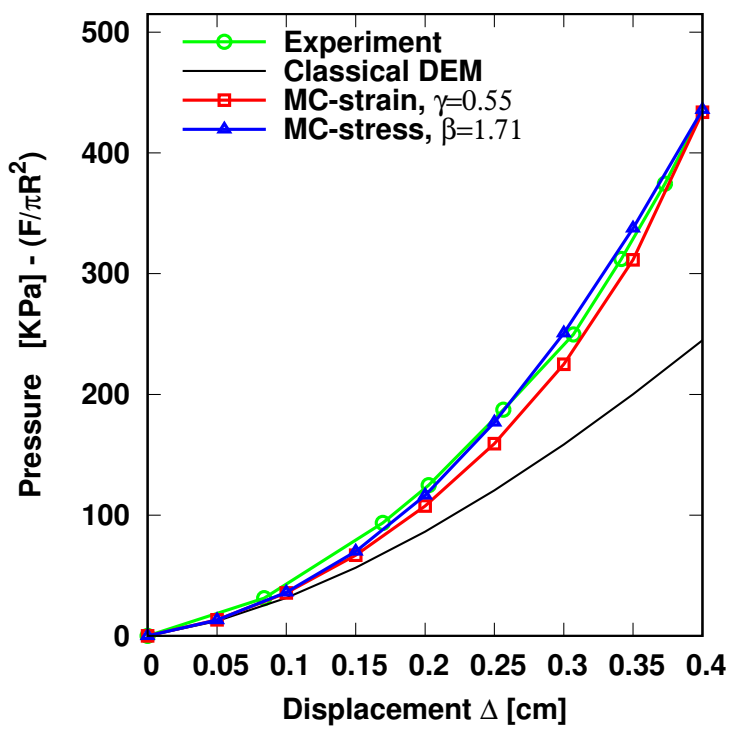

Figure 5.9: Validation of the mutli-contact formulation with experimental data of a rubber spheres compressed between two rigid plates [237].

these parameters must be calibrated in a way to not only capture the maximum force exerted on the top plate but also to follow the path during the sample compression and decompression.

A good agreement between experimental and simulation data is obtained for MC-stress model by choosing $\beta=1.65$ and shown in Fig. 5.10.b. Although, setting $\gamma=1.12$ which is the best fitting parameter for the case of MC-strain model, as suggested by Ref. [34], shows a good agreement but the representation is not good enough. Table. 5.2 gives the maximum force obtained by different models. It also is clear that selecting a higher prefactor $\gamma=1.22$ for the MC-strain model does not give the maximum force as obtained from the experimental data.

Furthermore, all contact forces between particles were visualized to establish the network of force chains. Figs. 5.13 and 5.14 shows all the contact forces between particles, for the initial configuration and for the maximum engineering strain at $13.4 \%$. Each contact force is drawn as a line, with the color of the line representing the magnitude of the normal force. The difference between 
force networks achieved by the classical and multi-contact implementations is clear, with higher normal forces for the multi-contact implementations. Distributions of normal contact forces are also different between the two multicontact models.

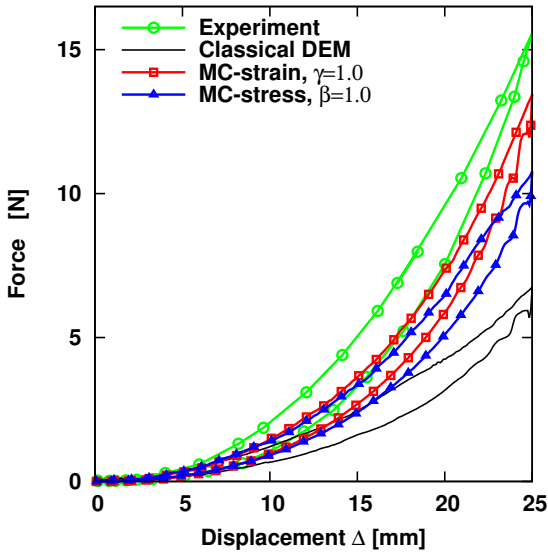

(a)

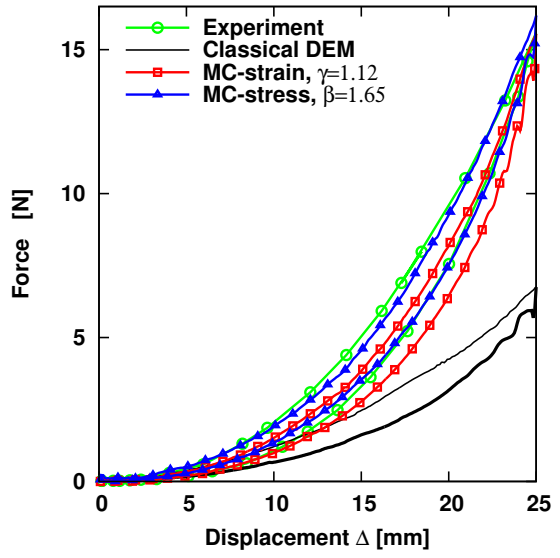

(b)

Figure 5.10: Force exerted on the top plate during compression of hydrogel box with prefactors of (a) $\beta, \gamma=1$, and (b) $\beta=1.65, \gamma=1.12$

\subsubsection{Compression using rubber spheres}

In addition to the hydrogel simulations, we perform a numerical study employing rubber particles in order to see the performance of the multi-contact models in case of harder material which brings a higher level of confinement. Simulation setup is identical to the previous test with the only difference in the maximum strain level, up to $28 \%$. The force displacement plot for a box filled with rubber particles is shown in Fig. 5.11. As expected, the MC-stress provides a higher maximum force with respect to the MC-strain and typical DEM.

\subsubsection{Compression using glass beads}

The system considered here is a cylinder, with a diameter of $12 \mathrm{~mm}$ and a height of $10.5 \mathrm{~mm}$, in where 17 glass beads with a mean diameter of $3.9 \mathrm{~mm}$ (min: 3.8 


\begin{tabular}{ll}
\hline Model & Max. force \\
\hline Experiment & $15.65[\mathrm{~N}]$ \\
Classical & $6.69[\mathrm{~N}]$ \\
MC-strain $(\gamma=1)$ & $13.29[\mathrm{~N}]$ \\
MC-stress $(\beta=1)$ & $10.95[\mathrm{~N}]$ \\
MC-strain $(\gamma=1.12)$ & $15.48[\mathrm{~N}]$ \\
MC-strain $(\gamma=1.22)$ & $18.59[\mathrm{~N}]$ \\
MC-stress $(\beta=1.65)$ & $16.07[\mathrm{~N}]$
\end{tabular}

Table 5.2: Maximum force exerted on the top plate during the compression at the maximum strain level.

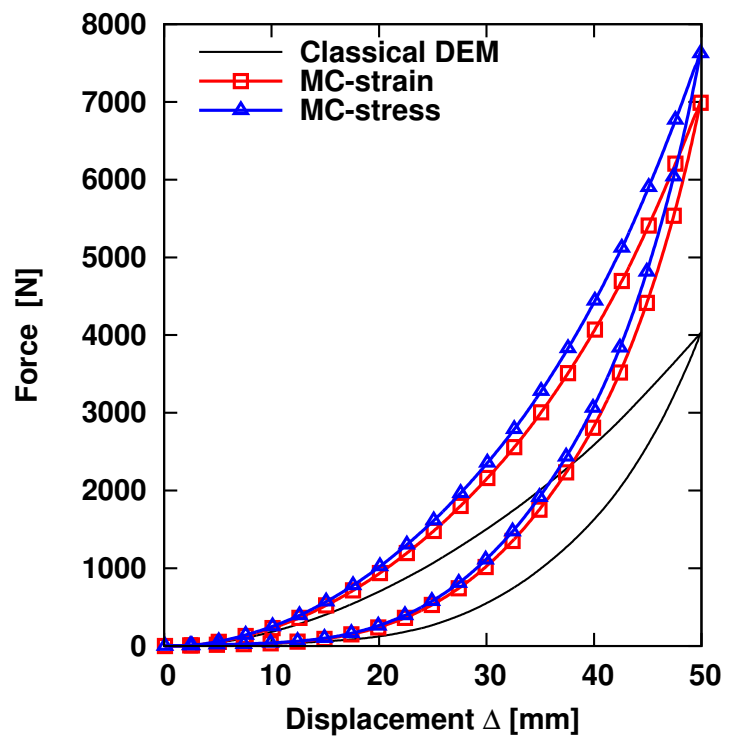

Figure 5.11: Force exerted on the top plate for the compression test of rubber balls.

$\mathrm{mm}$, max: $4.0 \mathrm{~mm}$ ). Glass properties applied for the simulations are shown in table. 5.1.The objective of this case is show how multi-contact

models are performing while compressing stiff materials under high stresses and 
compare the results with our generated experimental data. We have to note that since glass beads are brittle and for the purposes of this work we focused only the elastic part of the loading curve.

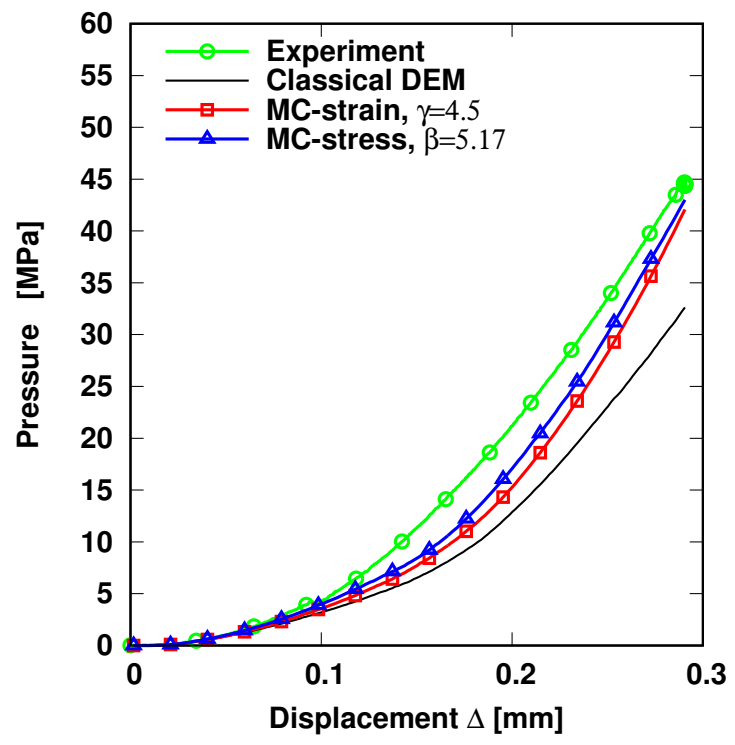

Figure 5.12: Pressure as a function of granule bed height for experimental and simulated compressions of glass beads.

Fig. 5.12 shows the results obtained from experimental and simulated compression of a cylinder filled with glass beads. Interestingly, we can see that the classical DEM is failing to resemble experimental data even for really small deformations (maximum strain $2.6 \%$ ) when it comes to really stiff materials. The MC-strain model with a prefactor of $\gamma=4.5$ offers a very good performance. The MC-stress model with a prefactor of $\beta=5.17$ offers a slightly better performance and it is able to capture the stronger non-linearity for this case of high stresses. We should note that prefactors $\gamma$ and $\beta$ where set in high values that is explained by the fact that for, MC-stain model, the new generated overlaps are in inverse proportion with the material Young's modulus (Eq. 5.5). As for the MC-stress model, and as we can see from Eq. 5.8, new generated forces are depending on the contact area between interacting particles. Hence, 
a stiffer material will result in less contact area.

\subsection{Computational cost performance of Mutli- contact models}

Lastly, one of the advantage of the proposed multi-contact model (MC-stress) with respect to MC-strain is its computational time which is quite faster than MC-strain. For this reason, we address the computational time obtained during the uni-axial compression using different approaches in table. 5.3. It is not surprising that the classical DEM is much faster than the other two multi-contact models due to its simplicity, i.e. considering one-to-one contacts during its simulations. The success comes into the comparison of MC-stress and MC-strain models where the presented approach is faster than the MC-strain model.

\begin{tabular}{llll}
\hline Model & T-hydrogel [s] & T-rubber [s] & T-glass [s] \\
\hline Classical & 38 & 84 & 22 \\
MC-strain & 668 & 1568 & 45 \\
MC-stress & 507 & 1143 & 34 \\
\hline
\end{tabular}

Table 5.3: Computational time of uni-axial compression using of differet approaches.

\subsection{Conclusions and outlooks}

In this research, we studied the importance of multi-contact models to describe the behavior of dense granular materials. At first, a brief introduction on the classical DEM was given. After that, the most common multi-contact model (MC-strain), which is based on the strain field around a particle, was explained. On this basis, we proposed a new approach to consider the effect of surrounding particles based on the stress tensor applied on a particle (MC-stress).

Incorporating the multi-contact correction in classical DEM significantly improves the comparison results (force-displacement) of dense packings. The behavior of hydrogel (soft material) particles under uni-axial compression/decompression and the behavior of a single compressed rubber 


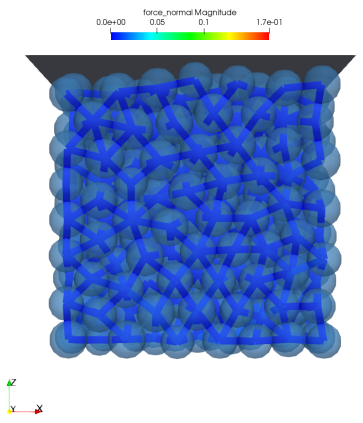

(a)

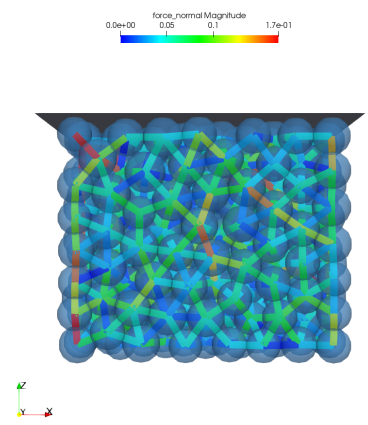

(b)

Figure 5.13: (Color online) Contact forces between particles of the particle assembly. The color of the force represents the magnitude of normal force, with light (red online) corresponding to large forces down to blue (blue online) representing zero forces. Network of force chains a) initial configuration b) normal contact forces computed at the maximum engineering strain with classical hertz contact model

sphere was successfully described with the new multi-contact approach (MCstress). Similar to MC-strain model, we included a prefactor $\beta$ which must be carefully calibrated depending on the type of the material.

One of the interesting achievements with the new multi-contact approach was its ability to provide a higher force at a given displacement with respect to the classical DEM and MC-strain. To confirm this feature, we tested our simulations using glass properties (stiff material) to reach a high level of confinement and the stronger non-linearity at higher stresses. This outcome can be employed in pharmaceutical industry for tableting processes, where reaching extreme confinement using DEM is a challenge. In addition to the given remarks, we showed the simplicity of the new multi-contact model, which makes the model easy to implement and faster in comparison to existing multi-contact model (MC-strain).

Next, we will conduct different types of experiments using a variety of materials (from soft to stiff) in order to allow for a wider comparison between our proposed model and experimental data. One can think of investigating the dependence of prefactors with respect to shape and mechanical properties of particles such as the elasto-plastic behaviour and the stress level experienced by 


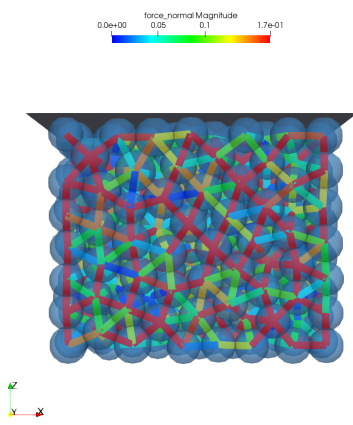

(a)

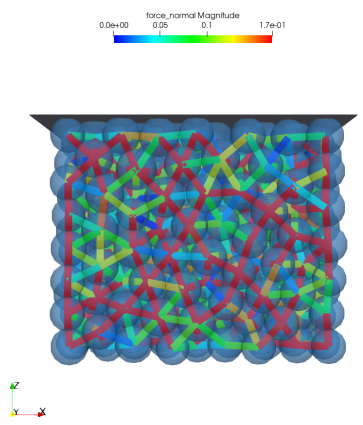

(b)

Figure 5.14: (Color online) Contact forces between particles of the particle assembly. The color of the force represents the magnitude of normal force, with light (red online) corresponding to large forces down to blue (blue online) representing zero forces. Network of force chains for normal contact forces computed at the maximum engineering strain with a) MC-strain contact model with $\gamma=1.12 \mathrm{~b}$ ) MC-stress contact model with $\beta=1.65$

the particles. Since the prefactors always have to be calibrated, it would be an interesting study to provide these parameters for simulation inputs an idea is to take the advantage of detailed finite elements simulations or to calibrate the meso-model from more refined approaches, where the deformation of particles is fully resolved.

\section{Acknowledgment}

Financial support for the study was provided by grant FP7 (ITN 607453) from the European-Union Marie Curie Initial Training Network, T-MAPPP, see http://www.t-mappp.eu/ for more information. 


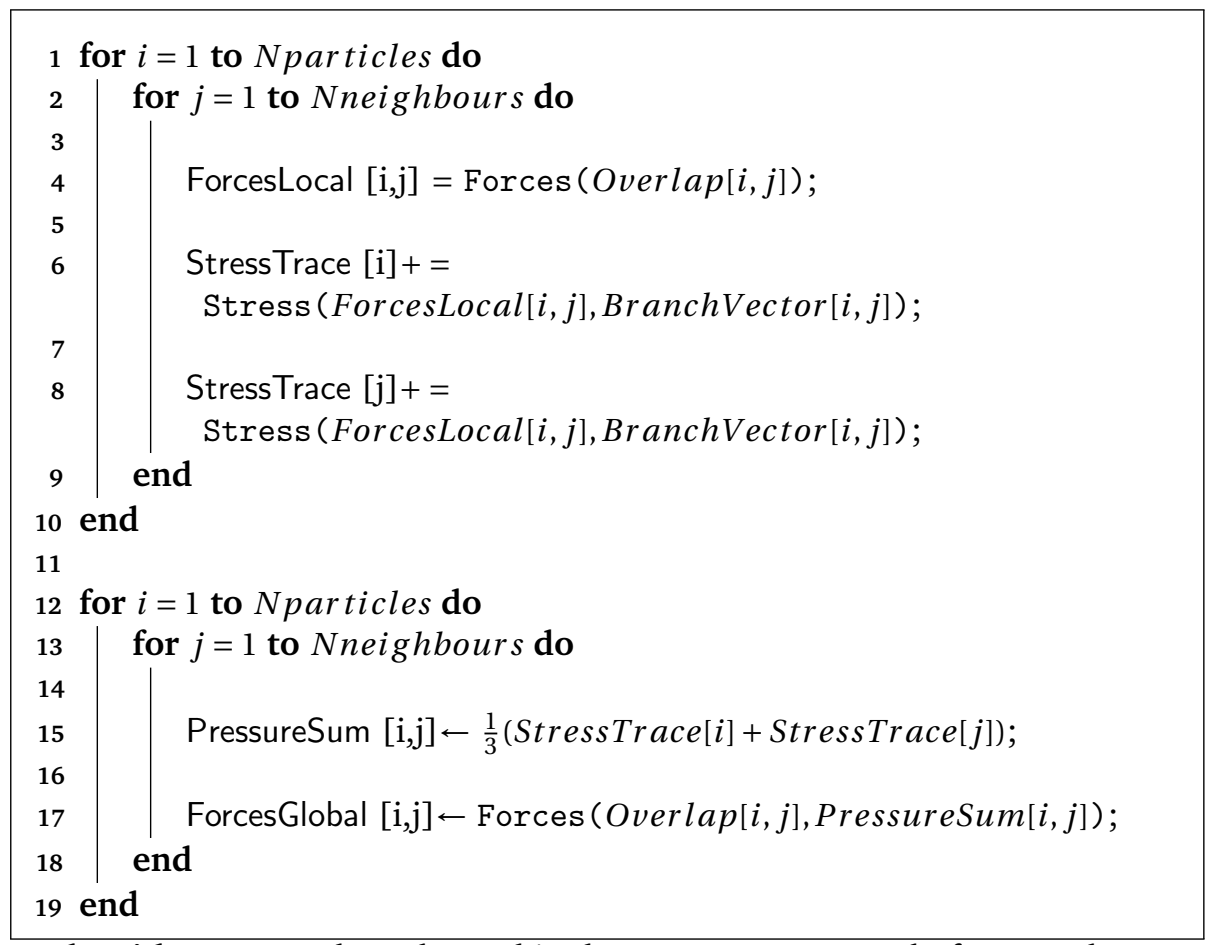

Algorithm 1: Pseudo-code used in the LIGGGHTS-DEM platform to obtain the global force acting on a particle.

\section{Appendix A: Algorithm used in the LIGGGHTS-DEM platform}

\section{Appendix B: Velocity Verlet integration scheme}

$$
\begin{aligned}
\mathrm{v}_{i+\frac{1}{2}} & =\mathrm{v}_{i}+\frac{\delta t}{2 m} f_{i} \\
\mathrm{x}_{i+1} & =\mathrm{x}_{i}+\mathrm{v}_{i+\frac{1}{2}}
\end{aligned}
$$

Calculate contact forces $=f_{i+1}$

$$
\mathrm{v}_{i+1}=\mathrm{v}_{i+\frac{1}{2}}+\frac{\delta t}{2 m} f_{i+1}
$$




\section{Chapter 6}

\section{Effect of Mass Disorder on Bulk Sound Wave Speed: A Multiscale Spectral Analysis}

But all this world is like a tale we hear, Men's evil, and their glory, disappear.

Abolghasem Ferdowsi

Energy transfer is one of the essentials of mechanical wave propagation (along with momentum transport). The diffusive (scattering) characteristics of energy during wave propagation is focus of many ongoing investigations. Predicting the energy propagation characteristics in real and wavenumber space through disordered (simplified) model, granular media like chains can assist in understanding the overall properties of wave propagation through real inhomogeneous media like soil; this eventually, can assist in seismic prospecting, non-destructive testing or designing metamaterials. The effect of disorder on energy propagation is first examined using an impulse propagating in a disordered granular chain, where disorder is given by the standard deviation of the mass distribution of the elements/granules. The use of an ideal one-dimensional granular chain allows isolating longitudinal P-wave mode from shear or rotational modes during analysis. From the total energy signals, it is observed that stronger disorder leads to more rapid attenuation of the signal. An ordered granular chain exhibits ballistic propagation 
of energy and a disordered granular chain exhibits diffusive-like propagation of energy. Disorder causes localization of energy near the source, where the intensity of the localization exhibits a power law like relationship with distance from the source and also increases quadratically with increase in disorder. Second, the effect of disorder on energy transfer across wavenumbers is examined using a standing sinusoidal wave initial condition (with a specific wavenumber). Disorder causes localization of energy near the particles with the lowest masses in the chain; higher disorder causes a faster decay of higher wavenumbers, whereas lower wavenumbers remain in the system for longer time period. Using the ensembled spatio-spectral energy response from the granular chain, the coefficients of a reduced complexity, disorder specific Master Equation are evaluated, which eventually can be used for mean-field macroscopic/continuum analyses. ${ }^{1}$

\subsection{Introduction}

Disorder in granular materials (like soil) can manifest in many ways from grain level to system level (contact disorder, geometrical disorder, asphericity, layering, etc.). All may have an effect on the mechanical wave transmission through the granular material in it's own unique way (for instance, contact disorder due to tiny polydispersity can reduce the mechanical wave speed and the transport of high frequency waves [38, 132, 171, 172, 211]. Knowing these effects can aid us in numerous ways for subsurface exploration or for non-destructive testing of materials $[6,209,216,247]$. Thus, there is a need to study the effects of disorder individually and hence the focus of this article will only be on mass disorder, for which a 1D granular chain has been chosen so that the P-wave mode is isolated from shear or rotational modes. A mechanical wave propagating through this simplified model 1D granular medium is bound to suffer from multiple scattering $[1,7,181,252]$. However, regardless of scattering, linear waves preserve some coherence that manifest as intensity correlations [257]. The results obtained from the chain also represent attributes of both longitudinal P-waves (compressional) and S-waves (shear) in a 3D system as stated in $[131,166]$; the frequency filtering effects are very similar to those in a 3D system, as observed in [172]. All the more reason to study energy content and spectral energy response of the propagating wave [21, 45, 69, 112, 260].

Classical continuum theories and Effective Medium Theory experience difficulty in modeling wave propagation in the intermediate or high frequency range

\footnotetext{
${ }^{1}$ Shrivastava, R.K., Taghizadeh, K., \& Luding, S. Journal of Statistical Mechanics, submitted Sept. 2019.
} 
because of their inability to resolve the micro-structure of the material. Continuum numerical techniques like Finite Element Method (F.E.M), Finite Difference Method (F.D.M), etc. can be used to predict wave propagation characteristics only if the right parameters are used, which are often difficult to find. However, Discrete Element Method (D.E.M) [41], a numerical technique which takes into account the disordered micro-structure of the material and the nonlinear contact forces between the interacting constituent granules of the media. This microscopic description is detailed but also costly so that only small volumes can be modeled. Nevertheless, D.E.M can be used to obtain the parameters of stochastic meso-scale models [149]. These then eventually can be used for continuum, macroscopic wave propagation analyses, hence, paving way towards a statistical micro-informed macroscopic treatment of the problem [161, 272].

The dynamic wave propagation in a granular chain can be argued as a Markovian process, the initial waveform (displacement/velocity of the particles) and the granular chain properties (pre-compression, sizes/masses of the particles through which the mechanical wave propagates) are sufficient to construct/predict successively the waveform at later time intervals [87, 195, 213]. The transition probability functions of the Markovian processes can be written in the form of Chapman-Kolmogrov equation, one of the versions of this equation is the Master Equation [103]. Hence, a Master Equation can be used to represent the transfer of energy across wavenumbers during mechanical wave propagation.

Sect. 6.2 gives the micro-mechanical model of the granular chain with linearized Hertzian repulsive interaction force acting between the granules; two types of initial condition (impulse propagation condition and standing wave condition; Sect. 6.2.2 and Sect. 6.2.3) are used to analyze energy propagation with distance and across wavenumbers. Sect. 6.2.4 gives a brief description about the mass disorder used in the granular chain. Sect. 6.3 lists the equations used for computing the total energy responses both in real and wavenumber space. Sect. 6.3.2 formulates the Master Equation and proposes the procedures to evaluate the transfer rates of energy across wavenumber space (componenets of the transfer matrix). Results are discussed in Sect. 6.4 and final conclusions are presented in Sect. 6.5.

\subsection{Granular Chain Model}

A granular chain of mesoscopic granules/particles has been modeled using the Hertzian repulsive interaction potential (a good approximation for spherical 
particles $[130,212])$, the repulsive interaction force between adjacent particles $i$ and $j$ is $(j$ can be $i+1$ or $i-1)$,

$$
\tilde{F}_{(i, j)}=\tilde{\kappa}_{(i, j)} \tilde{\delta}_{(i, j)}^{3 / 2},
$$

where $\tilde{\kappa}_{(i, j)}$ is the dimensional inter-particle contact stiffness, $\tilde{\delta}_{(i, j)}$ is the dimensional dynamic inter-particle overlap and the $3 / 2$ exponent is due to the Hertzian potential. The granular chain has a high pre-confining force $\left(\tilde{\Delta}_{(i, j)}\right)$ which prevents opening and closing of contacts. This assists in modeling mechanical wave propagation across well established granular chains. Assuming an external pre-compressional force $\tilde{F}_{o}$ on the granular chain in mechanical equilibrium, the initial particle overlap is given by

$$
\tilde{\Delta}_{(i, j)}=\left(\frac{\tilde{F}_{o}}{\tilde{\kappa}_{(i, j)}}\right)^{2 / 3} .
$$

The dimensional dynamic overlap is written as $\left.\tilde{\delta}_{(i, j)}=\tilde{\Delta}_{(i, j)}+\tilde{u}^{(i)}-\tilde{u}^{(j)}\right)$, where $\tilde{u}^{(i)}$ and $\tilde{u}^{(j)}$ are the dimensional displacements of the particles $i$ and $j$, respectively. To obtain a non-dimensionalized equation of motion for particles, the physical parameters have to be scaled. The minimum number of scaling parameters required for arriving at a nondimensionalized equation of motion are characteristic mass $\left(\tilde{m}_{o}\right)$, characteristic stiffness $\left(\tilde{\kappa}_{o}\right)$ and a length scale $(\tilde{\ell})$. The length scale could be chosen from a variety of parameters such as the particle size, the driving amplitude or the characteristic initial static overlap $\left(\tilde{\Delta}_{o}=\left(\tilde{F}_{o} / \tilde{\kappa}_{o}\right)^{2 / 3}\right.$; when all $\tilde{\kappa}_{(i, j)}=\tilde{\kappa}_{o}$; uniform contact stiffness; $\tilde{\kappa}_{o}$ is related to $\tilde{m}_{o}$ by Eq. (6.47)). $\tilde{\Delta}_{o}$ is chosen as the length scale for analyses. The nondimensional mass $b^{(i)}=\tilde{m}^{(i)} / \tilde{m}_{o}$, the non-dimensional stiffness is $\kappa_{(i, j)}=\tilde{\kappa}_{(i, j)} / \tilde{\kappa}_{o}$ and the non-dimensional displacement is $u=\tilde{u} / \tilde{\Delta}_{o}$. Without new scaling parameters, this also defines the non-dimensional time $t=\tilde{t} / \tilde{t}_{c}$ where

$$
\tilde{t}_{c}=\sqrt{\frac{\tilde{m}_{o}}{\tilde{\kappa}_{o} \tilde{\Delta}_{o}^{1 / 2}}} .
$$

and the non-dimensional repulsive interaction force

$$
F_{(i, j)}=\frac{\tilde{t}_{c}^{2}}{\tilde{m}_{o} \tilde{\Delta}_{o}} \tilde{F}_{(i, j)}
$$

The non-dimensional equation of motion for particle $i$ is now given by

$$
\begin{aligned}
b^{(i)} \frac{\mathrm{d}^{2} u^{(i)}}{\mathrm{d} t^{2}} & =F_{(i-1, i)}-F_{(i, i+1)} \\
& =\kappa_{(i-1, i)}\left[\Delta_{(i-1, i)}-\left(u^{(i)}-u^{(i-1)}\right)\right]^{3 / 2}-\kappa_{(i, i+1)}\left[\Delta_{(i, i+1)}-\left(u^{(i+1)}-u^{(i)}\right)\right]^{3 / 2}
\end{aligned}
$$


Eq. (6.5) can be solved numerically using the Verlet integration scheme, it can be used for analyses related to nonlinear dynamics of particles (Hertzian). The non-dimensional stiffness $\left(\kappa_{(i, j)}\right)$ and the initial overlap are given by (App. A; [130])

$$
\kappa_{(i, j)}=\sqrt{\frac{2}{b^{(i)}+b^{(j)}}}\left(b^{(i)} b^{(j)}\right)^{1 / 6} \text { and } \quad \Delta_{(i, j)}=\kappa_{(i, j)}^{-2 / 3},
$$

\subsubsection{Linearized Equation of Motion}

$F_{(i, j)}=\kappa_{(i, j)} \delta_{(i, j)}^{3 / 2}$ can be expanded around the initial overlap $\Delta_{(i, j)}$ as

$$
F_{(i, j)}=\kappa_{(i, j)} \Delta_{(i, j)}^{3 / 2}+\frac{3}{2} \kappa_{(i, j)} \Delta_{(i, j)}^{1 / 2}\left(\delta_{(i, j)}-\Delta_{(i, j)}\right)+\frac{3}{8} \kappa_{(i, j)} \Delta_{(i, j)}^{-1 / 2}\left(\delta_{(i, j)}-\Delta_{(i, j)}\right)^{2}+\ldots
$$

If the amplitudes of displacement $\left(u^{(i)}\right)$ are small during mechanical wave propagation, the nonlinear terms can be ignored and

$$
F_{(i, j)}=\kappa_{(i, j)} \Delta_{(i, j)}^{3 / 2}-\frac{3}{2} \kappa_{(i, j)} \Delta_{(i, j)}^{1 / 2}\left(u^{(j)}-u^{(i)}\right) .
$$

Hence, the linearized equation of motion for particle $i$ becomes

$$
\begin{aligned}
b^{(i)} \frac{\mathrm{d}^{2} u^{(i)}}{\mathrm{d} t^{2}} & =\kappa_{(i-1, i)} \Delta_{(i-1, i)}^{1 / 2}\left[\Delta_{(i-1, i)}-\frac{3}{2}\left(u^{(i)}-u^{(i-1)}\right)\right] \\
& -\kappa_{(i, i+1)} \Delta_{(i, i+1)}^{1 / 2}\left[\Delta_{(i, i+1)}-\frac{3}{2}\left(u^{(i+1)}-u^{(i)}\right)\right],
\end{aligned}
$$

which can eventually be written as

$$
b^{(i)} \frac{\mathrm{d}^{2} u^{(i)}}{\mathrm{d} t^{2}}=\frac{3}{2} \kappa_{(i, i+1)}^{2 / 3}\left(u^{(i+1)}-u^{(i)}\right)-\frac{3}{2} \kappa_{(i-1, i)}^{2 / 3}\left(u^{(i)}-u^{(i-1)}\right) .
$$

There are $p=0, \ldots, N+1$ particles in the granular chain with $0^{\text {th }}$ and $(N+1)^{t h}$ particles as the boundaries of the chain such that $u^{(0)}=0$ and $u^{(N+1)}=0$. Alternatively, periodic boundaries are realized by using $N+1$ particles, equivalent to setting $u^{(0)}=u^{(N+1)} \neq 0$, that is also allowed to move, so all vectors and matrices get length $N+1$ with $2^{i}=N+1$, with $i$ integer. Eq. (6.10) results in $N$ equations which are assembled in a matrix form:

$$
\mathbf{M} \frac{\mathrm{d}^{2} \mathbf{u}}{\mathrm{d} t^{2}}=\mathbf{K u},
$$


where $\mathbf{M}$ is a diagonal matrix with $b^{(1)}, b^{(2)}, b^{(3)} \ldots . . b^{(N)}$ as diagonal elements, $\mathbf{K}$ has $-\frac{3}{2}\left(\kappa_{(i+1, i)}^{2 / 3}+\kappa_{(i-1, i)}^{2 / 3}\right)$ as diagonal, $\frac{3}{2} \kappa_{(i+1, i)}^{2 / 3}$ as superdiagonal and $\frac{3}{2} \kappa_{(i-1, i)}^{2 / 3}$ as subdiagonal elements, other elements of $\mathbf{K}$ are $0 . \mathbf{u}$ is the displacement vector containing $u^{(i)}$ as the elements. Assuming $\mathbf{A}=-\mathbf{M}^{-1} \mathbf{K}$, Eq. (6.11) becomes

$$
\frac{\mathrm{d}^{2} \mathbf{u}}{\mathrm{d} t^{2}}=-\mathbf{A u} \text {. }
$$

Using an ansatz $\mathbf{u}=\mathbf{u}^{\prime} \exp ^{I \omega t}$, Eq. (6.12) becomes an eigenvalue problem

$$
\mathbf{A u}=\omega^{2} \mathbf{u}
$$

The eigenvalues $\omega_{j}^{2}\left(\omega_{j}\right.$ are the natural frequencies) and the eigenvectors $\mathbf{s}_{(j)}$ of the matrix A represent the eigendomain of the dynamic granular chain. The set of eigenvectors $\left(\mathbf{s}_{(j)}\right)$ can be orthonormalized by the condition

$$
\mathbf{s}_{(i)}^{\mathrm{T}} \mathbf{M s}_{(j)}=\delta_{i j},
$$

where $\delta_{i j}$ is the Kronecker delta symbol. A matrix $\mathbf{S}$ is constructed using $\mathbf{s}_{(j)}$ as columns and arranged in such a manner such that their corresponding $\omega_{j}$ are in increasing order. $\mathbf{S}$ is an eigenbasis matrix and can be used for projecting $u$ into eigenspace by the relation

$$
\mathbf{z}=\mathbf{S}^{-1} \mathbf{u}
$$

where $\mathbf{z}$ is the displacement amplitude (per eigenmode) in eigenspace. Using the transformation $\mathbf{S}^{-1} \mathbf{A} \mathbf{S}=\mathbf{G}$, where $\mathbf{G}$ is a diagonal matrix with $\omega_{j}^{2}$ as the diagonal elements, Eq. (6.12) becomes

$$
\frac{\mathrm{d}^{2} \mathbf{z}}{\mathrm{d} t^{2}}=-\mathbf{G} \mathbf{z}
$$

The solution of this equation is given by (in eigenspace and real space, respectively)

$$
\mathbf{z}(t)=\mathbf{C}^{(1)} \mathbf{a}+\mathbf{C}^{(2)} \mathbf{b} \quad \text { or } \quad \mathbf{u}(t)=\mathbf{S} \mathbf{C}^{(1)} \mathbf{a}+\mathbf{S C}^{(2)} \mathbf{b},
$$

where $\mathbf{C}^{(1)}$ is a diagonal matrix with $\sin \left(\omega_{j} t\right)$ as diagonal elements, $\mathbf{C}^{(2)}$ is also a diagonal matrix with $\cos \left(\omega_{j} t\right)$ as diagonal elements, $\mathbf{a}$ and $\mathbf{b}$ are vectors which are determined from initial conditions $\mathbf{u}_{o}$ (initial displacement vector) and $\mathbf{v}_{o}$ (initial velocity vector).

$$
\mathbf{a}=\mathbf{H}^{-1} \mathbf{S}^{-1} \mathbf{v}_{o} \quad \text { and } \quad \mathbf{b}=\mathbf{S}^{-1} \mathbf{u}_{o},
$$

where $\mathbf{H}$ is a diagonal matrix with $\omega_{j}$ as the diagonal elements. Two different types of initial conditions have been used for different types of analyses in the upcoming sections, impulse propagation and standing wave analysis. 


\subsubsection{Impulse Propagation Condition}

The initial condition for impulse propagation requires $\mathbf{u}_{o}$ and $\mathbf{v}_{o}$ to be

$$
u^{(i)}(t=0)=0, \quad v^{(i \neq n)}(t=0)=0, \quad v^{(n)}(t=0)=v_{o},
$$

where $n$ is the particle number to which impulse is imparted. $v_{o} \ll 1$ (initial particle overlap $\Delta_{o}$ ) to avoid opening and closing of contacts or to maintain the validity of the linearized equations of motion (Sect. 6.2.1). The first and the center particles in a granular chain have been imparted with impulse for further analyses in Sect. 6.4.1. Eq. (6.19) is used to get

$$
\mathbf{a}=\mathbf{H}^{-1} \mathbf{S}^{-1} \mathbf{v}_{o} \quad \text { and } \quad \mathbf{b}=0 .
$$

Hence, the displacement and velocity of particle $i$ becomes

$$
\mathbf{u}=\mathbf{S C}^{(1)} \mathbf{H}^{-1} \mathbf{S}^{-1} \mathbf{v}_{\boldsymbol{o}} \quad \text { and } \quad \mathbf{v}=\mathbf{S} \mathbf{C}^{(2)} \mathbf{S}^{-1} \mathbf{v}_{\boldsymbol{o}} ;
$$

which is also written as:

$$
u^{(i)}(t)=v_{o} \sum_{j=1}^{N} \frac{S_{i j} S_{n j} \sin \left(\omega_{j} t\right)}{\omega_{j}} \quad \text { and } \quad v^{(i)}(t)=v_{o} \sum_{j=1}^{N} S_{i j} S_{n j} \cos \left(\omega_{j} t\right) .
$$

\subsubsection{Standing Wave Condition}

For studying standing waves in the (periodic) chain, an initial sinusoidal waveform is given to the chain in the form of $\mathbf{u}_{o}=u_{o} \sin \left(\mathscr{N} \frac{2 \pi p}{N+1}\right)$ (where $p=1, \ldots, N+1$ or $0, \ldots, N, \mathscr{N}=1, \ldots,(N+1) / 2$ specifies the particular tone of the standing wave) and where the sin can be replaced easily by a cos, since $\mathscr{N}=(N+1) / 2$ does not work with sin, and $\mathbf{v}_{o}=0 . u_{o} \ll 1$ (initial particle overlap $\Delta_{o}$ ) to avoid opening and closing of contacts or to maintain the validity of the linearized equations of motion (Sect. 6.2.1). $\mathbf{a}$ and $\mathbf{b}$ are given as:

$$
\mathbf{a}=\mathbf{H}^{-1} \mathbf{S}^{-1} \mathbf{v}_{o}=0 \text { and } \mathbf{b}=\mathbf{S}^{-1} \mathbf{u}_{o} .
$$

Hence, the displacement and velocity of the particles become:

$$
\mathbf{u}=\mathbf{S C}^{(2)} \mathbf{S}^{-1} \mathbf{u}_{o} \text { and } \mathbf{v}=-\mathbf{S} \mathbf{H} \mathbf{C}^{(1)} \mathbf{S}^{-1} \mathbf{u}_{o} ;
$$

which is also written as:

$$
\begin{aligned}
& u^{(i)}(t)=\epsilon \sum_{j=1}^{N} S_{i j} \cos \left(\omega_{j} t\right) \sum_{p=1}^{N} S_{p j} \sin \left(\mathscr{N} \frac{2 \pi i}{N+1}\right), \\
& v^{(i)}(t)=-\epsilon \sum_{j=1}^{N} \omega_{j} S_{i j} \sin \left(\omega_{j} t\right) \sum_{p=1}^{N} S_{p j} \sin \left(\mathscr{N} \frac{2 \pi i}{N+1}\right) .
\end{aligned}
$$




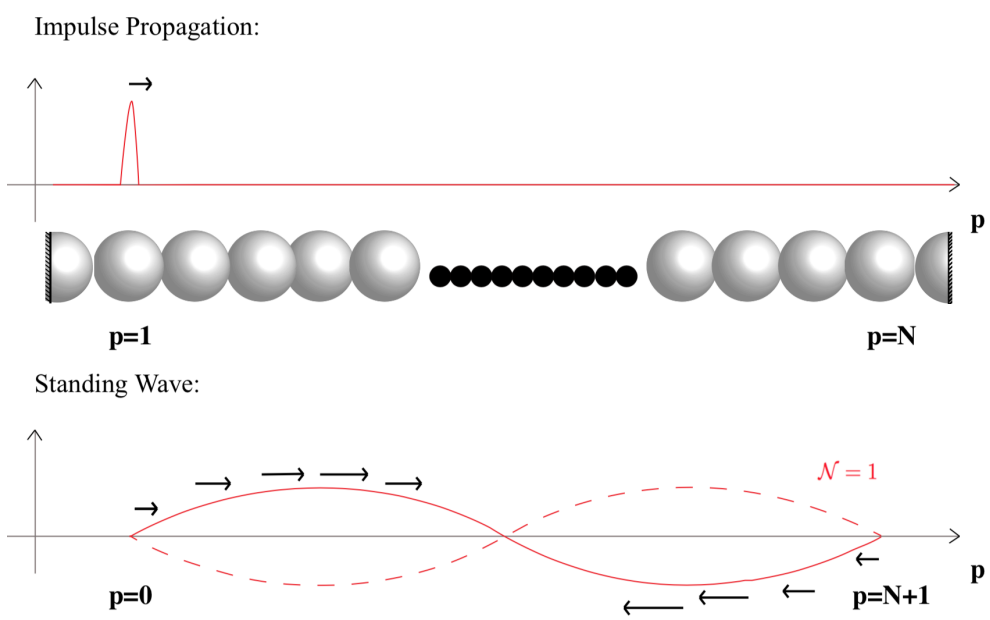

Figure 6.1: Impulse and (sin) Standing Wave initial conditions for a fixed-end and periodic granular chain, respectively.

For completeness, we note that the solution for arbitrary initial conditions is the superposition of Eqs. 6.21 and 6.24.

\subsubsection{Mass Disorder/Disorder Parameter $(\xi)$ \& Ensembles}

$b^{(1)}, b^{(2)}, b^{(3)}, \ldots b^{(N)}$, which are the diagonal elements of the mass matrix $\mathbf{M}$, have been selected from a normal distribution $f^{(n)}(b)=\frac{1}{\xi \sqrt{2 \pi}} e^{-\frac{(b-1)^{2}}{2 \xi^{2}}}$, the standard deviation $(\xi)$ quantifies the disorder parameter of the granular chain and the scaled average of the distribution is normalized to 1 [219]. A similar model has been used previously in $[131,218,219]$ for various wave propagation analyses. It was observed in [218] that the shape of the disorder probability (binary, normal, uniform or any other distribution) produces quantitatively similar wave propagation effects (frequency filtering, attenuation or mechanical wave velocities), up to a certain strength of disorder. The physical quantities (e.g. displacement, velocity, total energy, etc.) of multiple realisations of granular chains with a particular disorder parameter are averaged to obtain ensembled quantities, depicted by angular brackets $\langle\ldots\rangle$. 


\subsection{Energy Evolution}

For calculating the Kinetic Energy of individual elements/particles, we define the matrix $K E_{p q}$ where

$$
\mathbf{K E}=\frac{1}{2} \mathbf{M}[\mathbf{v} \otimes \mathbf{v}]=\frac{1}{2} \mathbf{M v v}^{\mathbf{T}} .
$$

The Kinetic Energy of individual elements/particles are the diagonal elements of the matrix KE, i.e. $K E_{P P}$ (capital letters $P P$ have been used as indices to denote the diagonal elements to avoid confusion with $p p$, which implies summation of the diagonal elements, i.e the trace of the matrix KE):

$$
K E^{(p)}(t)=K E_{P P}=\delta_{p P} \delta_{q P} K E_{p q}=\frac{1}{2} b^{(p)}\left(v^{(p)}(t)\right)^{2} .
$$

The Total Kinetic Energy is the trace of the matrix KE, i.e. $K E_{p p}$,

$$
K E T(t)=K E_{p p}=\delta_{p q} K E_{p q}=\frac{1}{2} \sum_{p} b^{(p)}\left(v^{(p)}(t)\right)^{2} .
$$

The Potential Energy of individual elements is basically the energy stored in the form of compression at the contacts of an element, it arises from the forces $\left(F^{(p)}\right)$ exerted by other elements in contact with the element whose potential energy is being examined. (For details please refer to App. B). For the Potential Energy, as well, we define the matrix

$$
\mathbf{P E}=-\frac{1}{2} \mathbf{K}[\mathbf{u} \otimes \mathbf{u}]=-\frac{1}{2} \mathbf{K} \mathbf{u} \mathbf{u}^{\mathbf{T}}=-\frac{1}{2} \mathbf{F} \mathbf{u}^{\mathbf{T}}, \quad \text { with } \quad \mathbf{F}=\mathbf{K u},
$$

where $\mathbf{K}$ is the stiffness matrix (Eq. (6.11)). The Potential Energy for individual elements are the diagonal elements of the matrix $P E_{p q}$,

$$
P E^{(p)}(t)=P E_{P P}=\delta_{p P} \delta_{q P} P E_{p q}=-\frac{1}{2} F^{(p)}(t) u^{(p)}(t) .
$$

The Total Potential Energy is the trace of the matrix $P E_{p q}$, i.e $P E_{p p}$,

$$
P E T(t)=P E_{p p}=\delta_{p q} P E_{p q}=-\frac{1}{2} \sum_{p} F^{(p)}(t) u^{(p)}(t) .
$$

The Total Energy of individual particles is the sum of its kinetic and potential energies. Using Eq. (6.26) and Eq. (6.29),

$$
\mathbf{T E}=\mathbf{K E}+\mathbf{P E}=K E_{p q}+P E_{p q} .
$$


Using Eq. (6.27) and Eq. (6.30),

$$
T E^{(p)}(t)=T E_{P P}=\delta_{p P} \delta_{q P} T E_{p q}=\frac{1}{2}(b(p))\left(v^{(p)}(t)\right)^{2}-\frac{1}{2} F^{(p)}(t) u^{(p)}(t) .
$$

We calculate the Energies (Potential Energy, Kinetic Energy and hence, Total Energy) relative to the initial pre-compressed state so that only the energy associated with wave propagation across the elements is taken into account. The equations (6.27),(6.28),(6.30) \& (6.31) are derived in App. B.

The center of total energy is defined as [7]

$$
R(t)=\frac{1}{T E_{t o t}} \sum_{p=1}^{N} p T E^{(p)}(t) \quad \text { with } \quad T E_{t o t}=\sum_{p=1}^{N} T E^{(p)}(t) .
$$

The mean squared width of the total system propagating and trapped wave is [7]

$$
r^{2}(t)=\frac{1}{T E_{t o t}} \sum_{p=1}^{N}(p-R(t))^{2} T E^{(p)}(t) .
$$

\subsubsection{Total Energy in the Wavenumber Domain}

TE or $T E_{p q}$ is in real space, to transform it into wavenumber space $\hat{\mathbf{T E}}$ or $\hat{T E_{k m}}$ ( $k$ and $m$ being rows and columns in wavenumber space), there is a need of change of basis as $\hat{\mathbf{E E}}=\mathbf{D F T}$ TE $\mathbf{D F T}^{-1}$, where $\mathbf{D F T}$ is the discrete Fourier transform matrix ${ }^{2}$, it can be used to calculate Fourier transform of vectors such that $\hat{\mathbf{u}}=\mathbf{D F T u}$ and $\hat{\mathbf{v}}=\mathbf{D T F v}$, where $\hat{\mathbf{u}}$ and $\hat{\mathbf{v}}$ are displacement and velocity vectors in wavenumber space, respectively. Hence,

$$
\hat{\mathrm{TE}}=\mathbf{D F T} \text { TE } \text { DFT }^{-1} .
$$

The diagonal of this matrix yields the total energy in wavenumber space per wavenumber $k$ :

$$
T E^{(k)}(t)=\hat{T E_{K K}}=\delta_{k K} \delta_{m K} \hat{T E_{k m}} .
$$

\footnotetext{
${ }^{2}$ DFT can be computed numerically as $\operatorname{dftmt}(\mathrm{x})$ matrix in Matlab, where $\mathrm{x}$ is the size of the square matrix DFT. Note that DFT is an orthogonal matrix, hence $\mathbf{D F T}{ }^{-1}=\mathbf{D F T}^{H}$, where superscript $H$ denotes conjugate transpose of a matrix.
} 


\subsubsection{Numerical Master Equation}

A Master Equation can be an efficient tool in analysing the criss-cross transfer of energy across different wavenumbers. In contrast to traditional methods of order reduction [66, 104, 137, 138, 192, 199], we do not focus on a subset of eigen-modes of the system, but group modes by wave-numbers (frequencies) to stochastically model the evolution in time (space), using only a much reduced set of frequency bands. The transfer of spatio-spectral energy is formulated as:

$$
\frac{\mathrm{d} e^{(r)}(t)}{\mathrm{d} t}=-Q_{r r}(t) e^{(r)}(t)+\sum_{r \neq s} Q_{r s}(t) e^{(s)}(t),
$$

$Q_{r r}$ depicts the energy loss (rate) from a particular wavenumber band $r$, which eventually gets transferred to all other wavenumber bands $s \neq r$. $Q_{r s}$ quantifies the transfer rates of energy back to the wavenumber band $r$ from $s$. $e^{(r)}(t)$ is the $r^{\text {th }}$ component of the binned energy vector $\mathbf{e}$ in wavenumber space. $\mathbf{e}$ is constructed by binning the spatio-spectral energy responses $\left(T E^{(k)}(t)\right)$. The binning is done by

$$
e^{(r)}(t)=\sum_{k=r-\Delta k / 2}^{k=r+\Delta k / 2} T E^{(k)}(t),
$$

where $\Delta k$ is the bandwidth of the bin and $r$ is the central wavenumber. The total energy is conserved, hence,

$$
\sum_{r=1}^{B} e^{(r)}(t)=\sum_{k} T E^{(k)}(t),
$$

where $B$ is the total number of bins assigned in wavenumber space. The binned spectral energy is normalized as

$$
\hat{e}^{(r)}(t)=\frac{e^{(r)}(t)}{\sum_{r=1}^{B} e^{(r)}(t)} .
$$

The coefficients $Q_{R R}$ and $Q_{r s}$ can be assigned as the diagonal and nondiagonal elements of the transfer matrix $Q$, respectively. Eq. (6.38) is thereby written in the scaled matrix form as

$$
\frac{\mathrm{d} \hat{\mathbf{e}}}{\mathrm{d} t}=\mathbf{Q} \hat{\mathbf{e}} .
$$




\section{Computing the elements of matrix Q:}

The elements of the matrix $\mathbf{Q}$ can be computed numerically using the boundary condition in Sect. 6.2.3, a standing wavemode $k$ belonging to a particular wavenumber band $r$ can be agitated in the form of a sinus (or cosinus) initial condition (Eq. (6.25)). The energy decay from normalized $e_{o}=1$ can be fitted by $\left\langle e^{(r)}(t)\right\rangle=\left(1-y_{1}\right) e^{-x_{1} t}+y_{1}$, where $x_{1}=Q_{r r}$ and $y_{1}$ represents the long term saturation value (the brackets $\langle\ldots\rangle$ indicate that ensembling is used to improve the quality of the fit); similarly, $Q_{r s}$ is determined by fitting $\left\langle e^{(s)}(t)\right\rangle=x_{2}\left(1-e^{-y_{2} t}\right)$, where $Q_{r s}=x_{2} y_{2}$ and $x_{2}$ represents the long term saturation value. Furthermore, one can apply Taylor expansion on the given fitting expressions. Considering the first term, avoiding higher orders, we can rewrite the expressions as: $\left\langle e^{(r)}(t)\right\rangle \approx\left(1-x_{1} t\right)$ and $\left\langle e^{(s)}(t)\right\rangle \approx x_{2} y_{2} t$. Hence, the energy decay of a particular wavenumber (band) as the energy spreads to other bands can be fitted by $\left\langle\hat{e}^{(r)}(t)\right\rangle=1-Q_{r r} t$ for small time intervals (before reaching the saturation regime); similarly, $Q_{r s}$ is determined by fitting $\left\langle e^{(s)}(t)\right\rangle=Q_{r s} t$. In the following, the linear and non-linear fit are called procedure 1 and 2, respectively ${ }^{3}$.

\subsection{Results and discussion}

An $N$ particles long granular chain has been used with Impulse and Standing Wave initial conditions for analyses. Sect. 6.4.1 deals with propagation of energy in a granular chain and the associated energy transfer. Sect. 6.4.2 deals with the analyses associated with energy transport between different wavenumbers.

\subsubsection{Energy Propagation with Distance}

Two types of impulse initial conditions are used. In one of the systems, the first particle $(N=1)$ has been imparted with initial velocity $v_{o}$. In the other system, the center particle has been imparted with $v_{o}$. Eq. (6.34) and Eq. (6.35) have been used for diffusion analyses associated with the impulse propagating in the granular chain.

\footnotetext{
${ }^{3}$ Note that the origin of time is not exactly at zero, due to swing in motion on the time scale of $t_{c}$. Fit quality is improved by allowing an additional parameter $t \rightarrow t-t_{0}$.
} 


\section{First Particle Excitation:}

Here, an $N=1024$ particles long granular chain is used. Particle $p=1$ is imparted with $v_{o}=0.01$. The time step for the computation is $\Delta t=0.1250$ and the maximum time evaluated is $t_{\max }=1024$, chosen in order to avoid reflection of the incident wave from the boundary. Figs. 6.2 and 6.3 display the ensembled total energy signal of four different disordered chains $\xi=0$ (Fig. 6.2(a)), $\xi=0.05$ (Fig. 6.2(b)), $\xi=0.1$ (Fig. 6.3(a)), $\xi=0.35$ (Fig. 6.3(b)). 500 different realizations of chains are used for ensembling. It is observed that there are two peaks in the energy signal for all instances of time shown, irrespective of disorder except for the ordered chain (Fig. 6.2(a)). The first peak is due to weak localization, a coherent backscattering effect during wave propagation near the source and the second peak is due to the propagating coherent wavefront (Fig. 6.3(a)). The ordered chain does not exhibit the weak localization peak because of absence of disorder. Higher $\xi$ shows a more rapid drop of the propagating coherent wavefront.
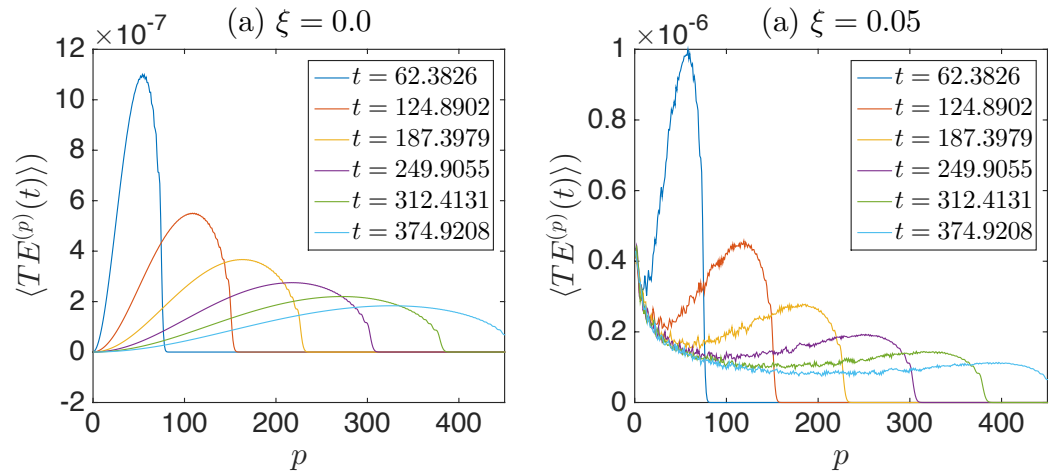

Figure 6.2: Ensembled energy signal at different instances of time for (a) ordered and (b) disordered chains.

Moreover, the weak localization peak decays with distance from the source, the total energy signals at all time instances collapse along this curve except the propagating wavefront which propagates along this long time limit decay curve.

Fig. 6.4 is the log plot of the decay curve associated with weak localization for chains (ensembled total energy signal at $t=875$ per particle; measurements are taken by limiting the space interval $(p \leq 800)$ to avoid propagating wave- 

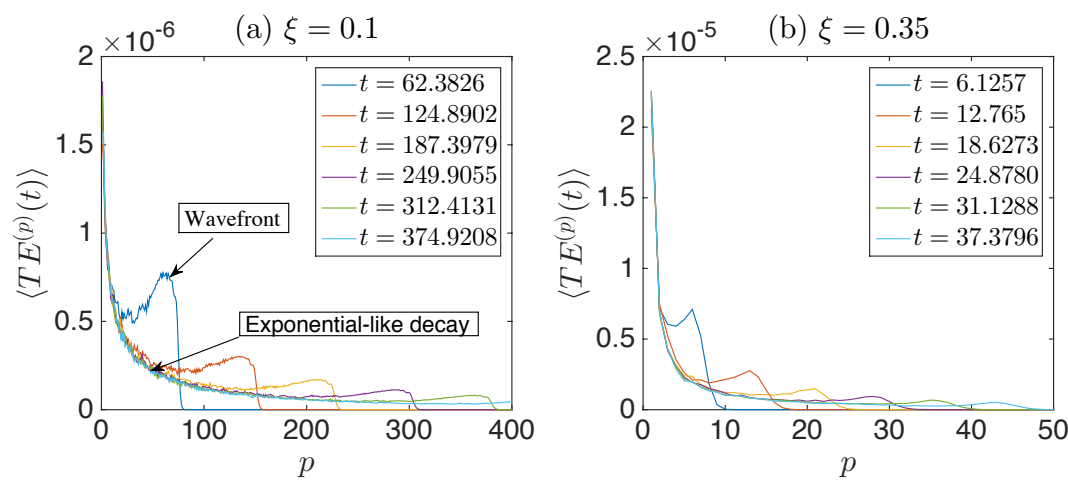

Figure 6.3: Ensembled energy signal at different instances of time for moderate and strongly disordered chains.

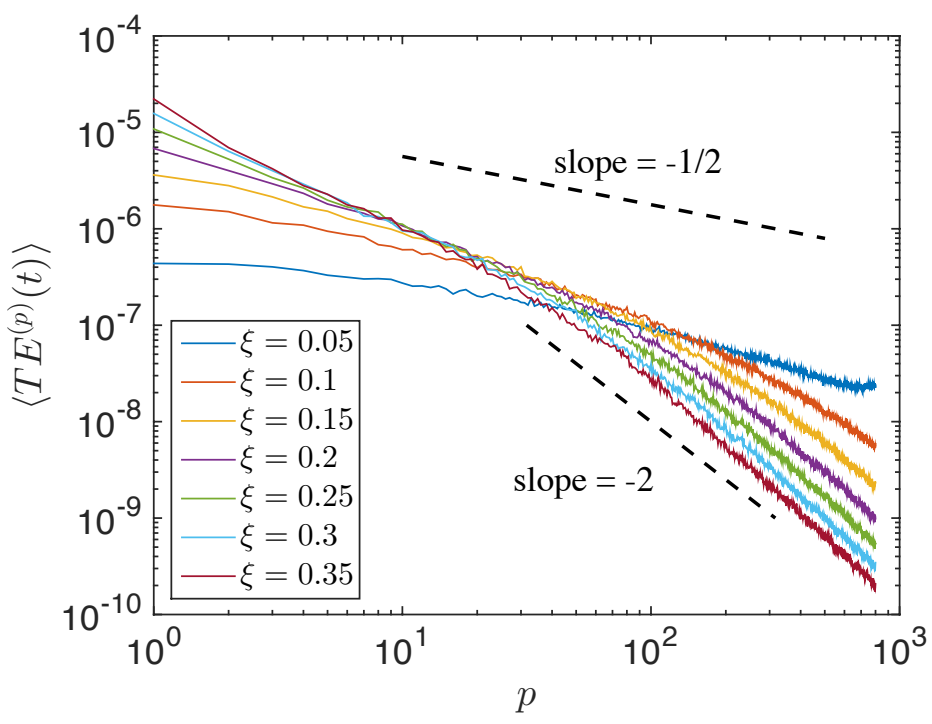

Figure 6.4: Power law relationship of the weak localization decay curve for different $\xi$, at later time $t \cong 875$. 

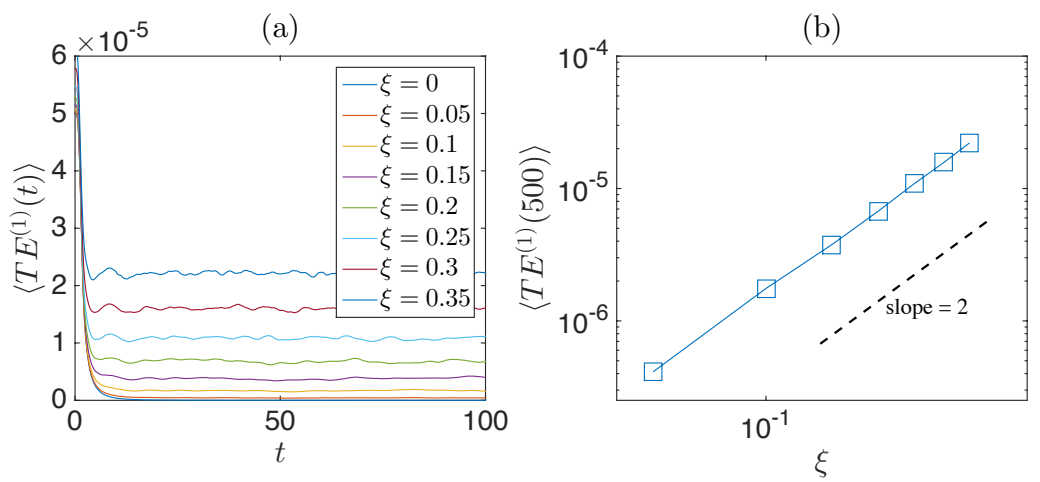

Figure 6.5: (a)Total Energy (averaged over 500 ensembles) of $p=1$ particle with time for chains with different $\xi$. (b)Almost constant residual energy localized in the first particle measured at $t \cong 500$

front), a power law relationship can be observed from the figure. It is observed that the rate of decay increases with increasing disorder parameter $\xi$ of the chain, indicating a stronger weak localization decay curve with increase in disorder. Fig. 6.5(a) shows the total energy signal of particle $p=1$ (source) with time. The figure shows that after the initial impulse, the energy of the source particle decays and becomes constant with very little fluctuations. This residual energy of the particle increases with increasing $\xi$, Fig. 6.5(b) shows a power law relationship of 2 between the disorder $\xi$ and the $T E$ of the first particle at long time $t=500$.

\section{Diffusion:}

The system used in the previous section $(N=1024$ particles long granular chain with $v_{o}=0.01$ imparted to the $1^{\text {st }}$ particle) is used in this sub-section as well. Eq. (6.34) is used to compute $\langle R(t)\rangle$ averaged over 500 ensembles. $\langle R(t)\rangle$ gives the averaged propagation of the center of energy, as plotted in Fig. 6.6. It shows that initially the center of energy does not propagate (as shown in the inset), during which the initial high frequency impulse is self-demodulated [246] by the granular chain (in contrast to a Gaussian pulse [7]). After this short time interval, the center of energy propagates with the same speed for different disorder parameters. $\xi=0.0$ has linear (ballistic) propagation of center of energy 
whereas, $\xi>0$ leads to nonlinear propagation of the center of energy with propagation speed decreasing with increasing time [218]. Stronger disorder yields smaller propagation speed with increase in time. Unlike $\xi=0.0$, higher $\xi$ result in the center of energy becoming confined in a finite space and this confinement space is smaller for stronger disorder. This occurs because $R(t)$ takes into account both the weak localization occurring close to the source and the propagating wavefront. The mean squared width of the energy during impulse

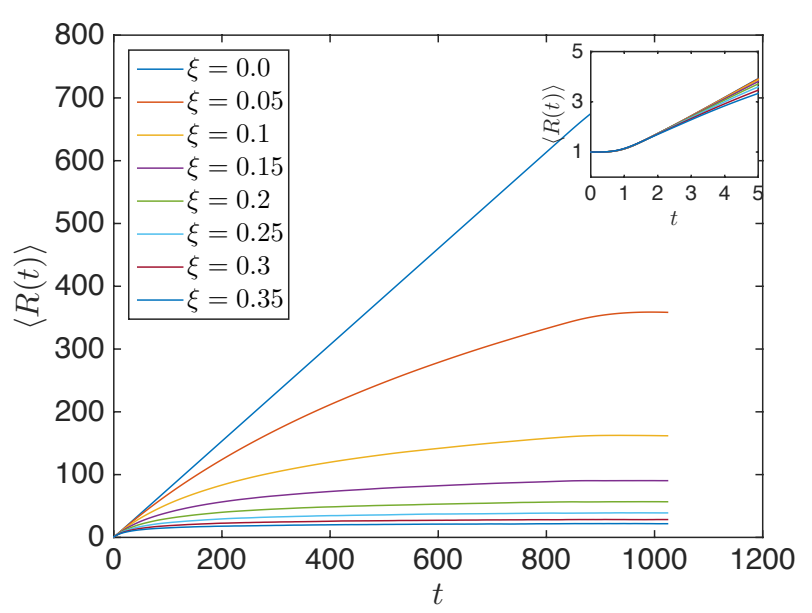

Figure 6.6: Position of the center of energy $(\langle R(t)\rangle)$ during impulse propagation for granular chains with different $\xi$.

propagation $r^{2}(t)$ is computed using Eq. (6.35), averaged over 500 realizations. Fig. 6.7 displays $\left\langle r^{2}(t)\right\rangle$ for granular chains with different $\xi$. It is observed that for an impulse response, the energy propagation is slightly superballistic for low disorder parameters (e.g. $\xi=0.05$ ) and gets nonlinear towards superdiffusive, diffusive and then subdiffusive for high disorder parameters.

\section{Center Particle Excitation:}

In order to ensure that the localization of energy during wave propagation is occurring near the source and is not a boundary effect, the effect of a different initial condition on impulse propagation in a granular chain is examined, $p=$ 


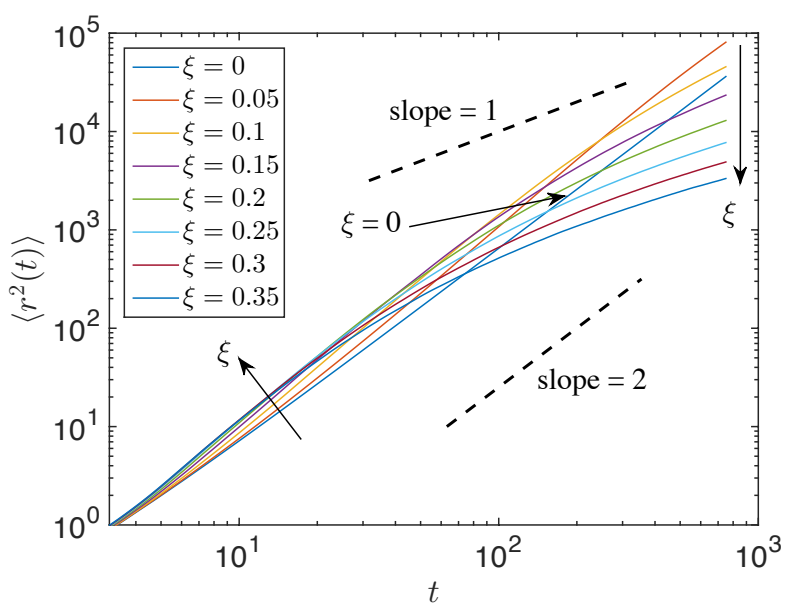

Figure 6.7: $\left\langle r^{2}(t)\right\rangle$ for different $\xi$ in log-log scale. Line is a guide to the eye. Arrows indicate the trend for $\xi>0$.

$1025^{\text {th }}$ particle of $N=2049$ particles long granular chain is imparted with $v_{o}=$ 0.01 . The time step for the computation $\Delta t=0.2501$ and $t_{\max }=1024$. Fig. 6.8 shows the total energy signal per particle at a particular instance of time $t \cong 750$ before the wave reach the end of the system for $\xi=0.1$ (Fig. 6.8(a)) and $\xi=0.3$ (Fig. 6.8(b)). It can be observed that the energy is localized around the source (the middle particle) and the two propagating wavefronts are moving in the opposite direction. The figure is symmetric around the center particle, which means we are in linear regime and tension and compression wave have same speed.

\subsubsection{Energy propagation in space and time}

With the goal to understand the evolution of standing waves in time, an initial sinusoidal waveform $\left(\mathbf{u}_{o}=u_{o} \sin \left(\mathscr{N} \frac{2 \pi p}{N+1}\right)\right.$; Sect. 6.2.3) is imparted to an $N+1=256$ particles long granular chain with $u_{o}=0.01$ and different $\xi$. The evolution of displacement and energy responses of particles/elements are then analyzed. Fig. 6.9 shows the evolving displacement of particles for the $\mathscr{N}=1$ standing wave in an ordered $(\xi=0.0)$ and a disordered chain $(\xi=0.3)$. Fig. 6.9(a) 

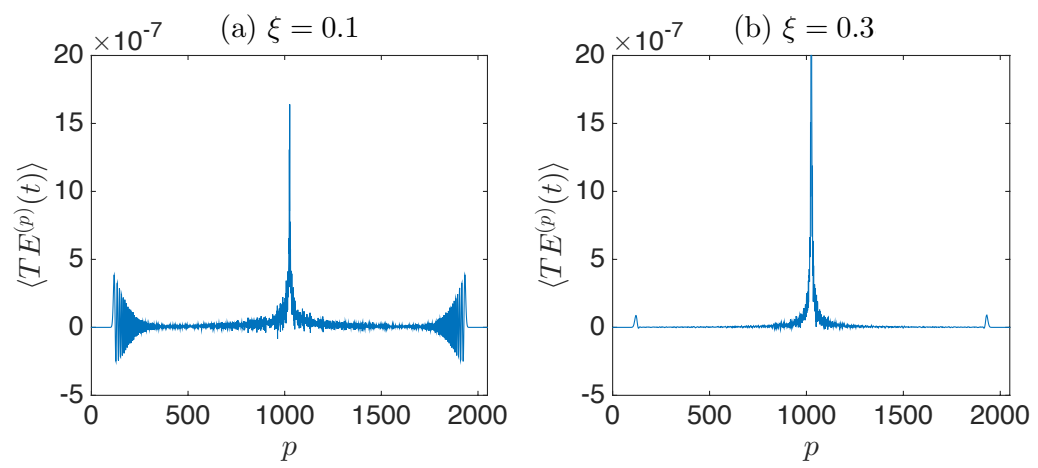

Figure 6.8: Center pulse ( $p=1025)$ is imparted with $v_{o}=0.01$ initially towards right. The total Energy signal is averaged over 500 ensembles at $t \cong 750$.

(a) $\xi=0.0$

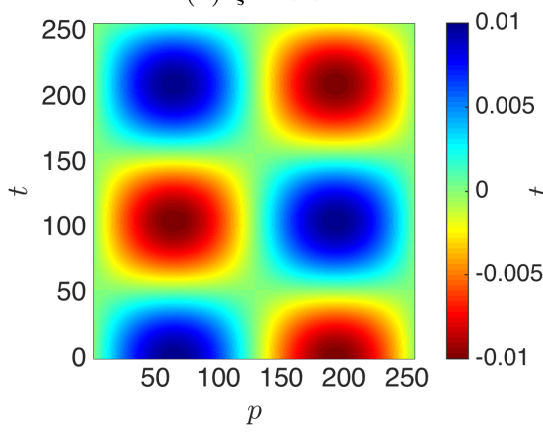

(b) $\xi=0.35$

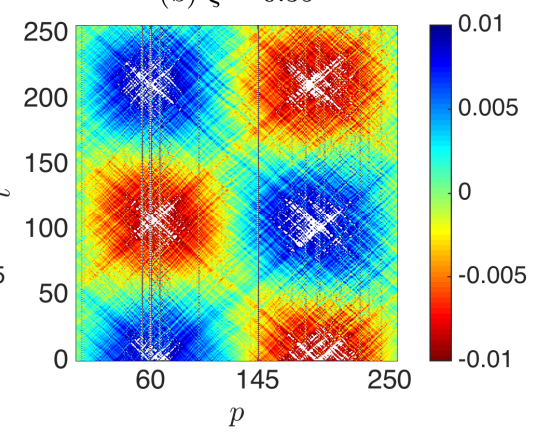

Figure 6.9: Sinusoidal standing wave (a) $u^{(p)}(t)$ ( $p=1$ to 256) of an ordered granular chain $(\xi=0.0)$. (b) $u^{(p)}(t)$ of a disordered granular chain $(\xi=0.3)$, while color indicates the largest displacements. 
displays the particles (color signifies the displacement amplitude) performing a standing wave motion $(\xi=0.0)$. However, in Fig. 6.9(b), it is observed that particles exhibit a perturbed standing wave motion of fluctuating low amplitude (color) in addition with traveling waves, clearly indicating that the disorder in the chains is disrupting the standing wave motion. It can also be observed that there are few localized high amplitude displacements shown by certain particles like $p=60$ and $p=145$; these particles are the lowest and the third lowest mass particles in the granular chain, in addition $p=60$ is close to a peak of the standing wave.
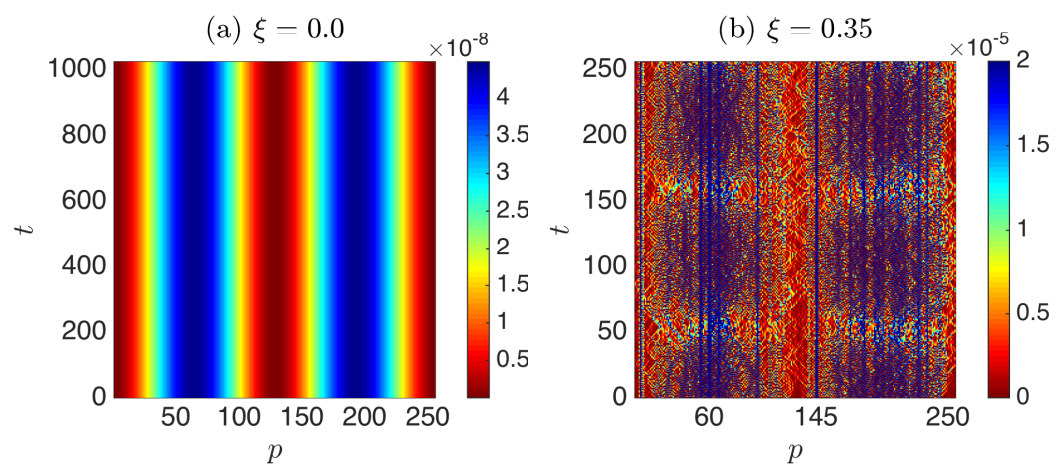

Figure 6.10: total energy per particle (a) $T E^{(p)}(t)(p=1$ to 256) of an ordered granular chain $(\xi=0.0)$. (b) $T E^{(p)}(t)$ of a disordered granular chain $(\xi=0.3)$, from the same data as in Fig. 6.9.

Fig. 6.10 (a) shows the total energy of the particles for the ordered $(\xi=$ 0 ) and disordered $(\xi=0.3)$ granular chains of Fig. 6.9 (color represents the amplitude $\left.T E^{(p)}(t)\right)$. Unlike Fig. 6.10(a), Fig. 6.10(b) exhibits localized high energy particles $p=145$ and $p=60$ (low mass particles), indicating localization of energy due to presence of disorder.

\subsubsection{Energy propagation across wave numbers}

Eq. (6.37) is used to obtain Total Energy in wavenumber space, then Eq. (6.41) is used to bin the energy responses accordingly. The number of bins used for the computation here onwards is $B=32$ with a bandwidth $\Delta k=\pi / 32=0.0982$. Fig. 6.11 shows the temporal evolution of total energy in wavenumber space 
for $\xi=0.3$ (color scale in the plot is $T E^{(k)}(t)$ ), $\mathscr{N}=90$ (Fig. 6.11 (a)) and 38 (6.11 (b)). A peak is initially observed at the initially agitated wavenumber $\left(k_{\text {ins }}=\mathscr{N} \frac{2 \pi}{N+1}\right)$, the peak decreases as the time progresses, the decay rate is lower for lower wavenumber, which can be observed when Fig. 6.11 (a) and (b) are compared. Fig. 6.12 displays the binned total energy in binned wavenumber space for $T E^{(k)}(t)$ (from the same data as in Fig. 6.11 (a)), Fig. 6.12 (b) represents the same total energy response averaged over 100 ensembles. As can be seen, ensemble averaging of many realizations improves the quality of response. Hence, the following analysis use ensemble averaged samples.

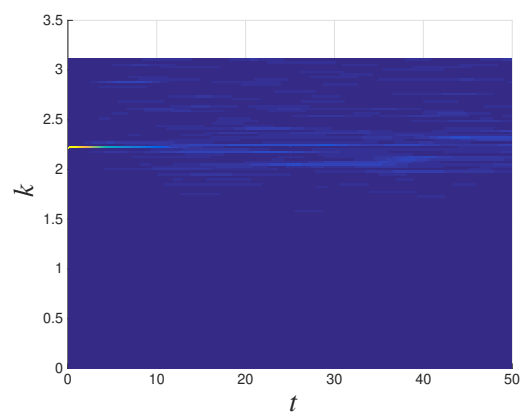

(a)

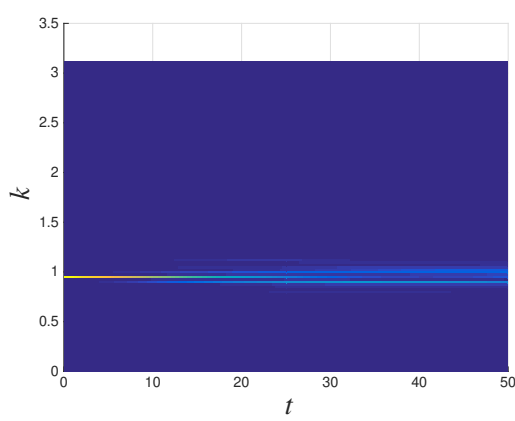

(b)

Figure 6.11: Total energy response $\left(T E^{(k)}(t)\right)$ in wavenumber space of a single realization of disordered granular chain $(\xi=0.3)$ initially agitated with (a) $k_{\text {ins }}=2.159(\mathscr{N}=90)$ and (b) $k_{\text {ins }}=0.981(\mathscr{N}=38)$.

In Fig. 6.13(a), the binned total energy response of the $23^{\text {rd }}$ band is plotted for six different disordered chains $(\xi=0.05,0.1,0.2,0.3,0.4$, and 0.5 ). Looking at this figure, one can see that the energy of the band is decaying faster for higher disorder and it reaches its saturation faster than the systems with lower disorder. In fact, this observation is expected since higher disorder in the system leads to faster loss of energy (attenuation) from the higher bands. Note that an ordered system $\xi=0$ does not show any decay of energy, since there is no singularity for diffusion of energy. Hence, its normalized energy will remain constant $(=1)$ with time. Fig. 6.13(b) shows the energy response of different agitated bands $(r(\mathscr{N})=5(18), 10(38), 15(58), 20(78), 25(98)$, and 30(118)) for a chain with disorder $\xi=0.3$. Higher bands $r$ (higher wave number $k$ ) lose more energy than lower bands. That means, when a lower band is agitated, lit- 


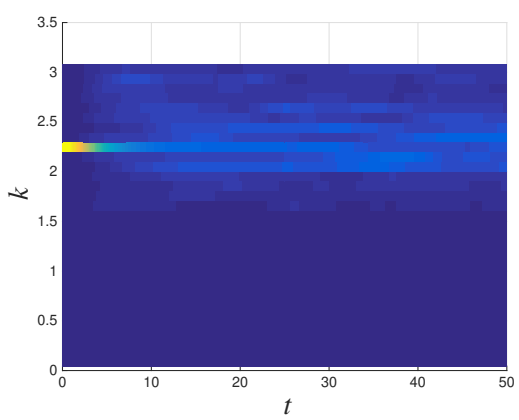

(a)

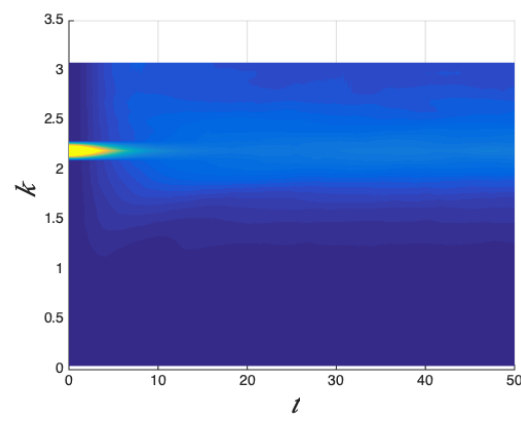

(b)

Figure 6.12: Binned total energy response (a) $\left(\hat{e}^{(23)}(t), k_{i n s}=2.159(\mathscr{N}=90)\right.$ of Fig. 6.11 (a) and (b) averaged over 100 ensembles $\left(\left\langle\hat{e}^{(23)}(t)\right\rangle, k_{i n s}=2.159(\mathscr{N}=90)\right.$.

tle energy is transferred to other bands. In contrast, agitation of a higher band leads to a significant energy transmission to other bands. The same behavior is seen for other disorders.

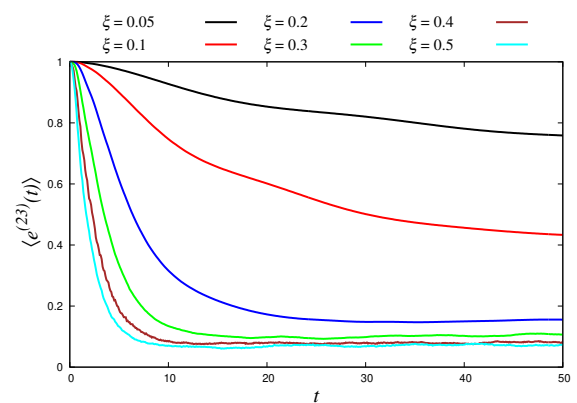

(a)

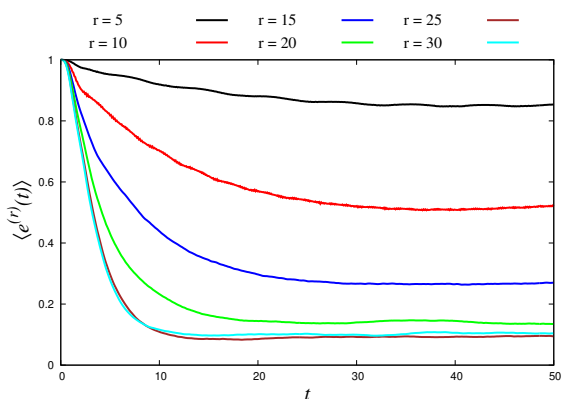

(b)

Figure 6.13: Normalized energy response of (a) $23^{\text {rd }}$ band for six different disordered chains, and (b) different bands, $r$, for the system with disorder $\xi=0.3$. 


\subsubsection{Attenuation}

The attenuation of seismic waves is an important property of particulate systems, which is of great interest to different communities [163]. Seismic attenuation can be quantified by the quality factor $Q[185,206,244]$, i.e. the transfer matrix shown earlier in Sect. 6.3.2. The knowledge of $Q$ is very desirable for improving seismic resolution, facilitating amplitude analysis, understanding the lithology of the subsurface better, and providing useful information about the porosity and fluid or gas saturation of reservoirs [101, 188]. Here, we first employ procedures explained earlier to obtain the components of the transfer matrix, then, we interpret our observations. Finally, we validate the proposed master equation using of the measured $\mathbf{Q}$ to solve the coarse, reduced model.

Fig. 6.14 (a) shows the ensembled decay rate of the bin which contains the initially agitated wavenumber $\left(r=23^{\text {rd }}\right.$ bin; $\left.k_{i n s}=2.159\right)$; the decay is well captured by the fit (Sect. 6.3.2) through procedure $1\left(Q_{r r}^{(1)}=0.2\right)$ and $2\left(Q_{r r}^{(2)}=0.1\right)$; superscript representing the procedures, see Sect. 6.3.2. As expected, damping coefficients obtained by linear and non-linear fits are consistent. In addition to the energy decay of the $r=23^{\text {rd }}$ bin, Fig. 6.14 (b) displays the rise of energy in a bin $(r=20)$ which is receiving energy from the $r=23^{\text {rd }}$ bin. The rise is well captured by the fitting procedures explained earlier (Sect. 6.3.2), where $Q_{r s}^{(1)}=0.1$ and $Q_{r s}^{(2)}=0.095$.

The energy of the bin which is initially agitated decays rather rapidly and achieves a steady state at $\left\langle\hat{e}^{(23)}(t)\right\rangle \approx 0.1$. On the contrary, the energy of the bin, which is receiving energy, starts increasing much slower and achieves a different steady state at $\left\langle\hat{e}^{(20)}(t)\right\rangle \approx 0.055$. Similar plots are shown for the $20^{\text {th }}$ bin in Fig. 6.15 (a) for $k_{i n s}=1.86$ and the rise of the $23^{\text {rd }}$ bin in Fig. 6.15 (b), where $20^{\text {th }}$ and $23^{\text {rd }}$ bins reach to their steady state at $\left\langle\hat{e}^{(20)}(t)\right\rangle \approx 0.18$ and $\left\langle\hat{e}^{(23)}(t)\right\rangle \approx 0.06$.

The transfer matrix $\mathbf{Q}$ can be deduced from wave-propagation simulations in disordered particle systems (in the ideal situation, without loss of generality, elastic and 1D). Using the set of $k_{\text {ins }}$ (where $\mathscr{N}=2,6,10, \ldots, 126$, one mode from every bin, hence, encompassing all the 32 bins) and the fitting equations mentioned in Sect. 6.3.2, the components of the transfer matrix are computed for ensembles of 100 disordered granular chains with $\xi=0.05,0.1,0.2,0.3,0.4$, and 0.5. Fig. 6.16 (a) depicts the diagonal elements of the $\mathbf{Q}$ matrix computed by procedure 1 (linear function). $Q_{r r}$ increases with increasing the bin number (i.e. increasing $k_{i n s}$ ) for different disorders. This can be attributed to the fact that lower bins/wavenumbers achieve a steady state faster, as it can be observed from Fig. 6.13 (b), leading to less energy loss. However, the increase of $Q_{r r}$ is 


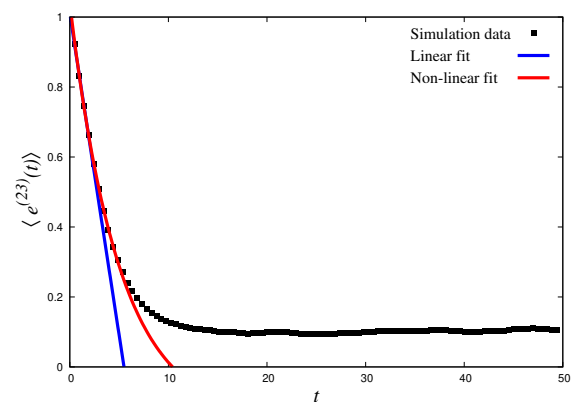

(a)

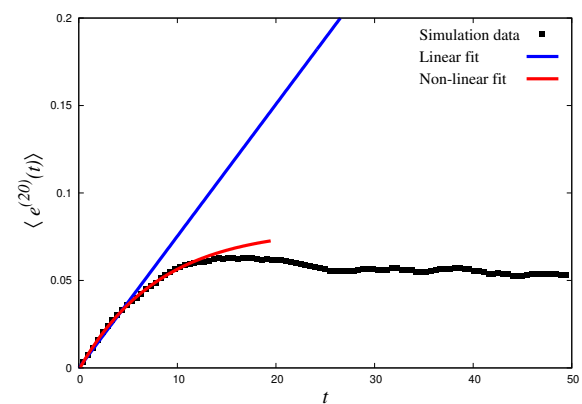

(b)

Figure 6.14: (a) Decaying binned total energy response of initially agitated bin 23 for $k_{\text {ins }}=2.159$ with the fits following procedures 1 and 2 . (b) Increasing binned total energy response of bin 20 receiving energy with the fits following procedures 1 and 2 . Only times from $2 \Delta t$ to $8 \Delta t$ have been used for the linear fit, and from $2 \Delta t$ to $18 \Delta t$ for the non-linear fit.

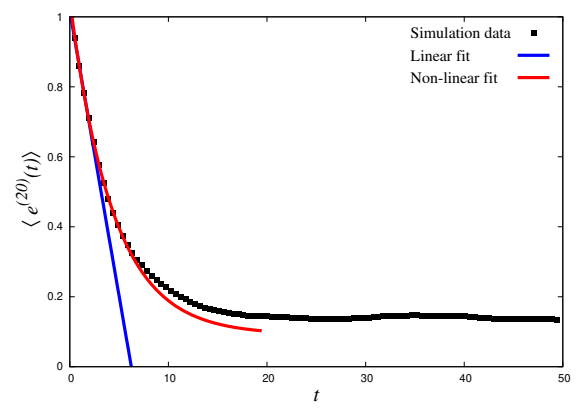

(a)

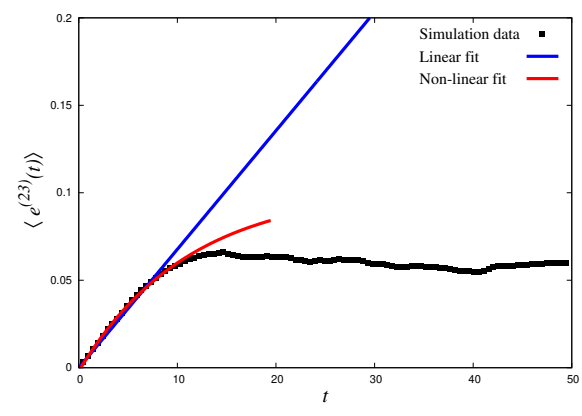

(b)

Figure 6.15: (a) Decaying binned total energy response of initially agitated bin 20 for $k_{i n s}=1.86$ with the fits following procedures 1 and 2 . (b) Increasing binned total energy response of bin 23 receiving energy with the fits following procedure 1 and 2 . Only times from $2 \Delta t$ to $8 \Delta t$ have been used for the linear fit, and from $2 \Delta t$ to $18 \Delta t$ for the non-linear fit.

faster in case of higher disordered. This reveals that the loss of energy is related to the disorder, i.e. attenuation increases with increasing the sample disorder. In addition, we plot the $Q_{r r}$ elements of $\mathbf{Q}$ derived using the non-linear function 
(procedure 2) in Fig. 6.16 (b). Like for the linear function observations, $Q_{r r}$ increases with increase of the bin number and disorder.

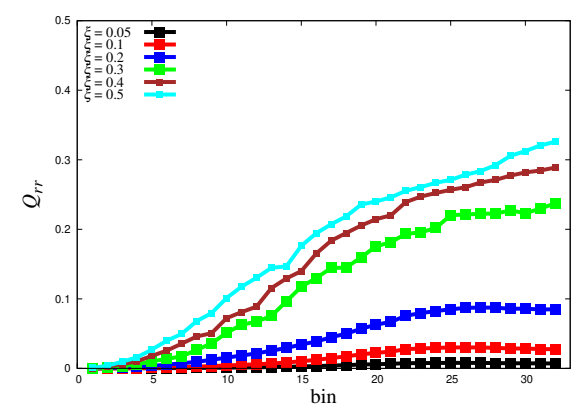

(a)

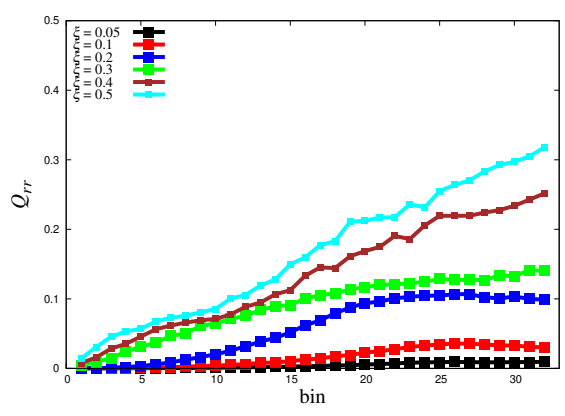

(b)

Figure 6.16: Diagonal components of $\mathbf{Q}$ obtained by (a) procedure 1(linear fit) and (b) procedure 2 (non-linear fit), for different disorders, as given in the inset.

Following the procedures explained earlier, off-diagonal components of $\mathbf{Q}$ are computed. Fig. 6.17 illustrates the color plots of $\mathbf{Q}$ computed by procedure 1 (linear fit) for six different disordered systems. Based on previous observations $[131,220]$, it was expected that the intensity of diagonal terms keeps increasing while the bin numbers increases, which means that higher bands attenuate faster, i.e. transfer more energy to their neighbors in comparison to the lower bins. On the non-diagonal, for low $s$ values one has very small probabilities for transfer of energy to another band, $r$, while for increasing $s$, the probability for energy-transfer to other bands $r$ increases, in particular there is also more transfer to the higher bands $r$.

\subsubsection{Reverse Modeling Frequency Propagation}

In the earlier section (Sec. 6.3.2), we developed the (reduced order) stochastic model based on the master equation

$$
\frac{d e^{(r)}(t)}{d t}=-Q_{r r} e^{(r)}(t)+\sum_{r \neq s} Q_{r s} e^{(s)}(t)-d_{r} e^{(r)}(t)
$$

that describes the evolution at time $t$ of the energy $e^{(r)}(t)$ in wave-number (frequency) band $k \pm \Delta k / 2$ by transfer to all other bands, with rate $Q_{r r}$, or from all 


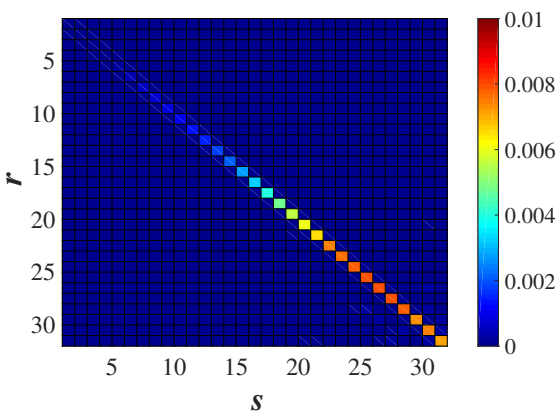

(a)

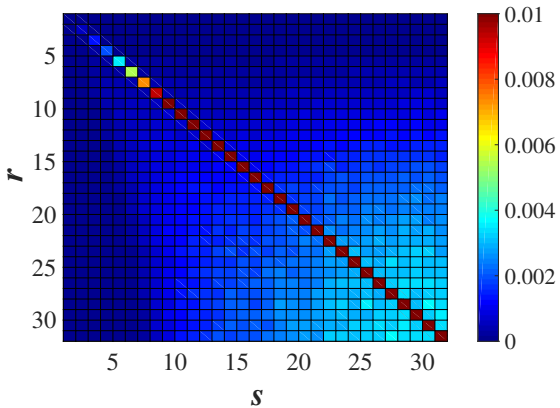

(c)

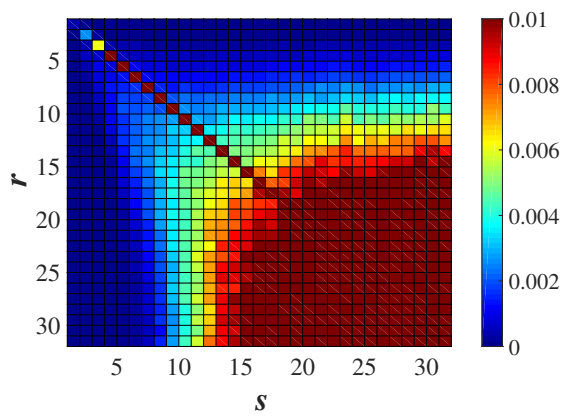

(e)

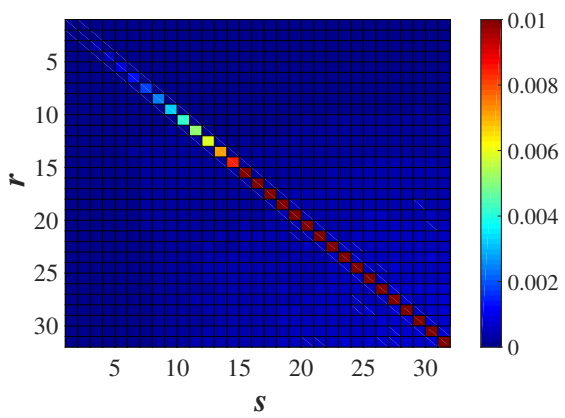

(b)

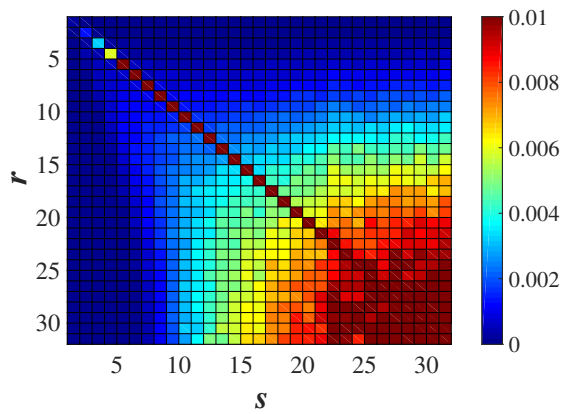

(d)

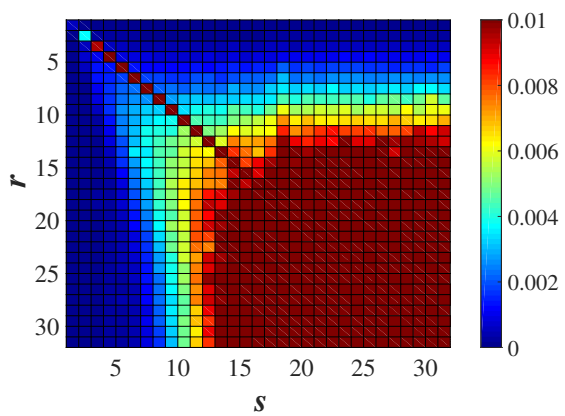

(f)

Figure 6.17: The transfer rate matrix $Q_{r s}$ computed through procedure 1 (linear fit, Sect. 6.3.2) for different disorders: (a) $\xi=0.05$, (b) $\xi=0.1$, (c) $\xi=0.2$, (d) $\xi=0.3$, (e) $\xi=0.4$, and (f) $\xi=0.5$, where the first index indicates the source $r$ and the second is the receiver target $s$. The diagonal elements $\left(Q_{r r}\right)$ are the attenuation coefficients of the total energy in band/bin $r$. The corresponding non-diagonal components $Q_{r s}$ are the increase rates of energy in bin $s$ by receiving energy from bin $r$. 
other bands $s$ into $r$ with rate $Q_{r s}$. Note that the sequence of indices in the transfer rates is relevant because $Q_{r s}$ is not symmetric. The last term in Eq. (6.43) represents damping, where frequency dependent damping (energy dissipation, not attenuation) is characterized by the damping rate $d_{r}$, which is zero in this research since the energy in a chain is considered to be conserved.

Given any matrix $\mathbf{Q}$ the evolution of energy with time can be easily modeled/integrated using the master equation in Eq. (6.43). Thanks to the reduced order modeling in a Master-Equation approach that combines many eigenmodes in frequency bands, this solution is very efficient but expresses the energy transfer between the bands qualitatively correctly.

After measuring the components of the transfer matrix in the previous subsection, here, we test the validity of the proposed master equation, Eq. (6.38). Using Eq. (6.43) and the $\mathbf{Q}$ matrix computed in Fig. 6.17.(d) (for the disordered system $\xi=0.3$ ), the frequency propagation of specific bins can be computed (the final purpose of the Master Equation formulation, here this computation can serve as cross-validation), which has been done for two different bins $10^{\text {th }}$ and $23^{\text {rd }}$ in Fig. 6.18. As expected, the higher frequency bin ( $23^{\text {rd }}$ bin) looses energy faster than the lower frequency bin ( $10^{\text {th }}$ bin) to other frequency bins/bands, it indicates that the lower frequency passes and the higher frequency attenuates, a fundamental frequency propagation characteristic in disordered granular media, as observed earlier, see Refs. [131, 174, 220].

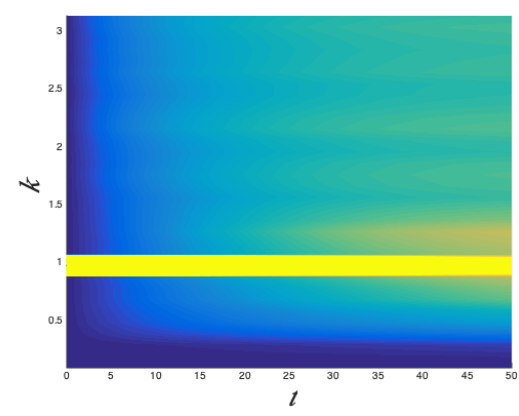

(a)

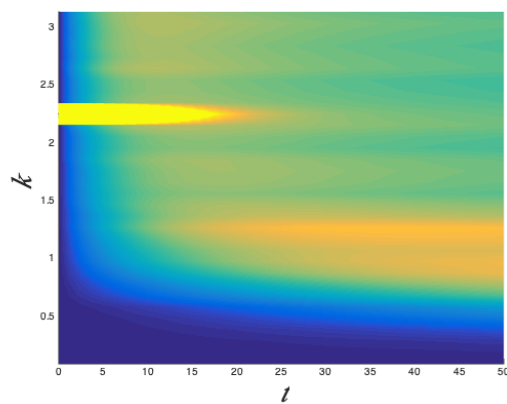

(b)

Figure 6.18: Frequency propagation of (a) $10^{\text {th }}$ and (b) $23^{\text {rd }}$ bins computed using the $\mathbf{Q}$ matrix from Fig. 6.17(d), for $\xi=0.3$. 


\subsection{Conclusion}

A mass disordered granular chain with linearized Hertzian repulsive interaction force between the granules is a model for disorder granular systems (Sect. 6.2). It is used for studying the energy propagation characteristics having two types of initial conditions:

- Impulse initial condition for studying energy propagation with distance (Sect. 6.2.2).

- Standing wave initial condition for studying energy propagation across wavenumbers with time (Sect. 6.2.3)

The results in Sect. 6.4 show the existence of twin peaks in the total energy signal plotted with distance from the source for disordered granular chains; unlike ordered chains which have only one peak. The peak near the source is attributed to weak localization due to disorder, whereas, the second peak is the propagating coherent wavefront. The weak localization decay curve is observed to be invariant with time and exhibits a power law like relationship with distance from the source, where the rate of decay increases with increasing disorder parameter $(\xi)$. However, the second peak (wavefront) rapidly decreases with distance from the source and more strongly with increasing $\xi$. The center of energy's propagation speed decreases with $\xi$. Disorder leads to a confinement of the center of energy within a finite space that decreases with increasing $\xi$. This is a sign of diffusive-like propagation of energy. It was found that the ballistic propagation of energy for an ordered chain becomes slightly superballistic for small disorder and then successively superdiffusive, diffusive and subdiffusive with increase in disorder $\xi$.

Sect. 6.4.2 focuses on the total energy evolution of the standing wave sinusoidal initial condition, as is used for studying the transfer of energy across wavenumbers in disordered chains. In real space, the energy becomes localized, around lower masses in the chain. In wave number spacer, the energy in higher wavenumbers decays faster than the energy in lower wavenumbers. The Master Equation for the transfer of energies across wavenumbers (bands) models a disordered granular chain easing the computational expense relative to the full model with all degrees of freedom, but keeping the characteristics of energy transfer across the wave numbers. Two procedures were introduced in Sect. 6.3.2 for computing the components of transfer matrix $\mathbf{Q}$ in the Master Equation while quantities all transfer rates. 
The Weak Localization effect and the diffusion model are interesting features of disordered media, which can be modeled and parametrized to understand and predict some of the material properties. The Master Equation acts as a stochastic model for modeling wave propagation in disordered media. Its coreingredient, the transfer matrix $Q$, can be improved with better statistics, fitting curves and better formulation, which may also incorporate nonlinear interactions between different wavenumber bands. There are some open issues in the fit procedure, the initial time interval to be used for fitting the decay of the agitated bin or the rise in energy of the non-agitated bin. One option would be to select the appropriate time interval according to the initially inserted wavenumber through the dispersion relation. As mentioned previously in Sect. 6.1, the computed Master Equation can be used for continuum analyses associated with larger systems, which contains information about the micro-structure in the form of components of the transfer matrix but needs to be calibrated and validate experimentally.

Future work will focus on two and three dimensional packings of granular materials by employing Discrete Element Method for numerical simulations. Also, the investigation can be carried out not only on size mass disorder but also stiffness disorder (material characteristic) where also different species, soft and stiff particles, are mixed. To pursue this goal, as first step, damping has to be added to the (reduced complexity) master equation and the equation can be tested for different materials; in experiments, we expect much stronger damping for softer (e.g. rubber) than for stiff (e.g. sand) particles. The masterequation can then be calibrated separately for pure stiff (almost elastic) and pure soft (strong damping) samples before one addresses. The challenge is defining the master equation for two (or more) types of materials. This will involve calibrating also the transfer-terms that quantify the transfer of energy between the species, where the characteristic time-scales (rates) of the species can be highly different (fast for stiff, vs. very slow for soft).

Another challenge for future research is to understand also non-linear terms in the master equation, that can be added in the form of, e.g. mixed quadratic terms in energy, which have shown to produce higher harmonics [174], and which are needed in order to properly predict band-gaps, transmission bands and possibly other non-linear interactions between different bands in the presence of both single or multiple materials. 


\section{Acknowledgment}

This work is part of the Industrial Partnership Programme (IPP) "Computational sciences for energy research" of the Foundation for Fundamental Research on Matter (FOM), which is part of the Netherlands Organisation for Scientific Research (NWO). This research programme is cofinanced by Shell Global Solutions International B.V. We acknowledge support from ESA under contract 4000115113 Soft Matter Dynamics.

\section{Appendix. A}

If Hertzian repulsive interaction forces between particles are taken into consideration, the contact stiffness is given by $([130,131])$

$$
\tilde{\kappa}_{(i, j)}=\tilde{Y}_{(i, j)}\left[\frac{\tilde{r}_{i} \tilde{r}_{j}}{\tilde{r}_{i}+\tilde{r}_{j}}\right]^{1 / 2},
$$

where

$$
\tilde{Y}_{(i, j)}^{-1}=\frac{3}{4}\left(\frac{1-v_{i}^{2}}{\tilde{E}_{i}}+\frac{1-v_{j}^{2}}{\tilde{E}_{j}}\right) .
$$

$\tilde{E}_{i}$ and $v_{i}$ are the elastic modulus and Poisson's ratio, respectively of the particle $i$. If the particles are made up of the same material, $\tilde{Y}_{(i, j)}$ becomes same for all the contacts,

$$
\tilde{Y}^{-1}=\frac{3}{2}\left(\frac{1-v^{2}}{\tilde{E}}\right) .
$$

The characteristic stiffness of the contact is

$$
\tilde{\kappa}_{o}=\frac{\tilde{E}}{1-v^{2}}\left[\frac{2 \tilde{m}_{o}}{243 \pi \tilde{\rho}}\right]^{1 / 6} .
$$

The characteristic initial overlap becomes

$$
\tilde{\Delta}_{o}=\left(\frac{\tilde{F}_{o}}{\tilde{\kappa}_{o}}\right)^{2 / 3} .
$$

The characteristic time is

$$
\tilde{t}_{c}=\frac{1}{\tilde{\Delta}_{o}^{1 / 4}} \sqrt{\frac{1-v^{2}}{\tilde{E}}}\left[\frac{243 \pi \tilde{\rho} \tilde{m}_{o}^{5}}{2}\right]^{1 / 12} .
$$


The scaled stiffness ratio is

$$
\kappa_{(i, j)}=\frac{\tilde{\kappa}_{(i, j)}}{\tilde{\kappa}_{o}}=\sqrt{\frac{2}{b^{(i) 1 / 3}+b^{(j) 1 / 3}}}\left(b^{(i)} b^{(j)}\right)^{1 / 6} .
$$

The initial overlap during static equilibrium can be formulated as

$$
\Delta_{(i, j)}=\frac{\tilde{\Delta}_{i, j}}{\tilde{\Delta}_{o}}=\kappa_{(i, j)}^{-2 / 3} .
$$

\section{Appendix. B}

Energy Equations (Kinetic, Potential and Total Energy) are used for analyzing the properties of mechanical wave propagation across the granular chain. The matrix form of the equations assists in computing the signals both in real and wavenumber space with ease. The equations (6.27),(6.28),(6.30) \& (6.31) are derived in this section using a three particles system $(i-1, i$ and $i+1)$. For better visualization, the pre-compressed granular chain is represented by a spring mass system as shown in Fig. 6.19. The springs are the interactions between the elements, resembling particle contacts.

Kinetic Energy: During the initial pre-compressed static, equilibrium state, the elements/particles are not in motion, hence their respective velocities are 0 , therefore, the Kinetic Energy is 0 . The Kinetic Energy associated with particle $p$ during wave propagation is given by

$$
K E^{(p)}(t)=\frac{1}{2} b^{(p)}\left(v^{(p)}\right)^{2} .
$$


Initial pre-compressed state :

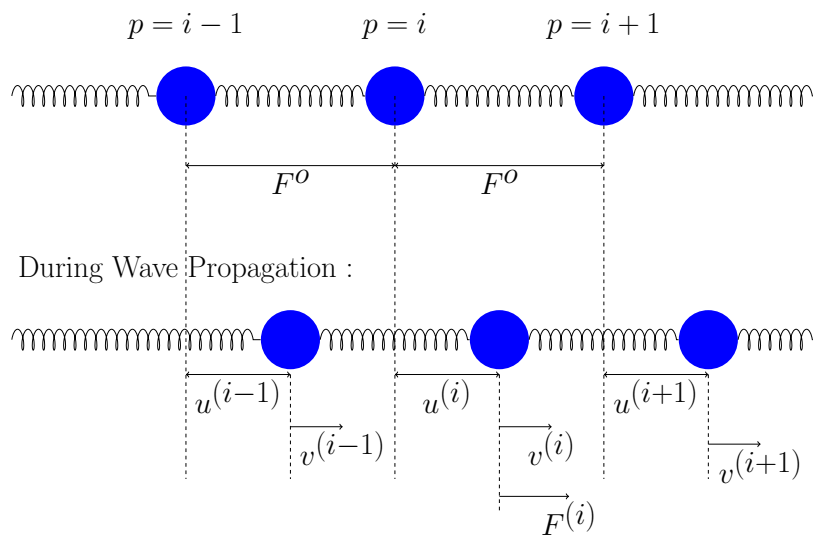

Figure 6.19: Particle chain for simple test cases of energy equations.

The Kinetic Energy can also be calculated by the use of matrices as in Eq. (6.27),

$$
\begin{aligned}
& \mathbf{K E}=\frac{1}{2} \mathbf{M}[\mathbf{v} \otimes \mathbf{v}]=\frac{1}{2} \mathbf{M v v} \mathbf{T}^{\mathbf{T}} \\
& =\frac{1}{2}\left(\begin{array}{ccccc}
\ddots & \vdots & \vdots & \vdots & \vdots \\
\ldots & b^{(i-1)} & 0 & 0 & \ldots \\
\ldots & 0 & b^{(i)} & 0 & \ldots \\
\ldots & 0 & 0 & b^{(i+1)} & \ldots \\
\vdots & \vdots & \vdots & \vdots & \ddots
\end{array}\right)\left(\begin{array}{c}
\vdots \\
v^{(i-1)} \\
v^{(i)} \\
v^{(i+1)} \\
\vdots
\end{array}\right)\left(\begin{array}{lllll}
\ldots & v^{(i-1)} & v^{(i)} & v^{(i+1)} & \ldots
\end{array}\right) \\
& =\frac{1}{2}\left(\begin{array}{ccccc}
\ddots & \vdots & \vdots & \vdots & \vdots \\
\ldots & b^{(i-1)}\left(v^{(i-1)}\right)^{2} & b^{(i-1)} v^{(i-1)} v^{(i)} & b^{(i-1)} v^{(i-1)} v^{(i+1)} & \ldots \\
\ldots & b^{(i)} v^{(i-1)} v^{(i)} & b^{(i)}\left(v^{(i)}\right)^{2} & b^{(i)} v^{(i)} v^{(i+1)} & \ldots \\
\ldots & b^{(i+1)} v^{(i-1)} v^{(i+1)} & b^{(i+1)} v^{(i)} v^{(i+1)} & b^{(i+1)}\left(v^{(i+1)}\right)^{2} & \ldots \\
\vdots & \vdots & \vdots & \vdots & \ddots
\end{array}\right)
\end{aligned}
$$


The diagonal elements of this matrix give the Kinetic Energy of individual elements (Eq. (6.52)) and the Total Kinetic Energy of the system is the trace of this matrix. The non-diagonal elements give the spatial velocity correlation of the elements with other elements in the system. For instance, $K E_{12}$ is the velocity correlation of the 1 st element with the 2 nd element.

Potential Energy: The Potential energy during the initial precompression state is due to the initial static overlap. However, during wave propagation the potential energy is calculated as

$$
P E^{(p)}(t)=\left(P E_{(i-1, i)}+P E_{(i, i+1)}\right) / 2 ;
$$

where $P E^{(p)}(t)$ is the potential energy of the individual particles, $P E_{(i-1, i)}$ and $P E_{(i, i+1)}$ are potential energies due to the adjacent springs (contacts). According to the definition of Potential Energy,

$$
P E_{(i, j)}=-\int\left(F_{(i, j)}-F_{o}\right) d x,
$$

where $F_{o}$ is the force between particles during initial static overlap, $F_{(i, j)}$ is the dynamic force between particles $i$ and $j$ and $x$ is the change in the length of the spring. Hence, using Hertzian contact forces (Eq. (6.5))

$$
\begin{aligned}
P E_{(i, j)} & =-\int_{\Delta_{(i, j)}}^{\Delta_{(i, j)}-u^{(i)}+u^{(j)}}\left[\kappa_{(i, j)}\left(\Delta_{(i, j)}-u^{(i)}+u^{(j)}\right)^{3 / 2}-\kappa_{(i, j)} \Delta_{(i, j)}^{3 / 2}\right] \mathrm{d} x \\
& =\frac{2}{5} \kappa_{(i, j)}\left[\Delta_{(i, j)}-u^{(i)}+u^{(j)}\right]^{5 / 2}-\frac{2}{5} \kappa_{(i, j)} \Delta_{i, j}^{5 / 2}-\kappa_{(i, j)} \Delta_{i, j}^{3 / 2}\left(u^{(j)}-u^{(i)}\right) \\
& =\frac{2}{5} \kappa_{(i, j)} \Delta_{(i, j)}^{5 / 2}\left[1+\frac{\left(u^{(j)}-u^{(i)}\right)}{\Delta_{(i, j)}}\right]^{5 / 2}-\frac{2}{5} \kappa_{(i, j)} \Delta_{i, j}^{5 / 2}-\kappa_{(i, j)} \Delta_{(i, j)}^{3 / 2}\left(u^{(j)}-u^{(i)}\right), \\
& \left(\text { Using Newton's expansion, }(1+x)^{m}=1+m x+m(m-1) \frac{x^{2}}{2 !}+\ldots\right) \\
& (\text { Neglecting higher order and lower coefficient terms }) \\
& \cong \frac{3}{4} \kappa_{(i, j)}^{2 / 3}\left(\left(u^{(i)}\right)^{2}-u^{(i)} u^{(i+1)}\right) .
\end{aligned}
$$


Hence, using Eq. (6.54)

$$
P E^{(p)}(t) \cong-\frac{3}{4}\left[\kappa_{(i-1,1)}^{2 / 3} u^{(i-1)} u^{(i)}-\left(\kappa_{(i-1, i)}^{2 / 3}+\kappa_{(i, i+1)^{2 / 3}}\right)\left\{u^{(i)}\right\}^{2}+\kappa_{(i, i+1)}^{2 / 3} u^{(i+1)} u^{(i)}\right] .
$$

The Potential Energy in the matrix form is computed as (Eq. (6.30))

$$
\begin{aligned}
& \mathbf{P E}=-\frac{1}{2} \mathbf{K}[\mathbf{u} \otimes \mathbf{u}]=\frac{1}{2} \mathbf{K u u ^ { T }}, \\
& =-\frac{1}{2}\left(\begin{array}{ccccc}
\ddots & \vdots & \vdots & \vdots & \vdots \\
\ldots & -\frac{3}{2}\left(\kappa_{(i-2, i-1)}^{2 / 3}+\kappa_{(i-1, i)}^{2 / 3}\right) & \frac{3}{2} \kappa_{(i-1, i)}^{2 / 3} & 0 & \ldots \\
\ldots & \frac{3}{2} \kappa_{(i-1, i)}^{2 / 3} & -\frac{3}{2}\left(\kappa_{(i-1, i)}^{2 / 3}+\kappa_{(i, i+1)}^{2 / 3}\right) & \frac{3}{2} \kappa_{(i, i+1)}^{2 / 3} & \ldots \\
\ldots & 0 & \frac{3}{2} \kappa_{(i, i+1)}^{2 / 3} & -\frac{3}{2}\left(\kappa_{(i, i+1)}^{2 / 3}+\kappa_{(i+1, i+2)}^{2 / 3}\right) & \ldots \\
\vdots & \vdots & \vdots & \vdots & \ddots
\end{array}\right) \\
& \left(\begin{array}{c}
\vdots \\
u^{(i-1)} \\
u^{(i)} \\
u^{(i+1)} \\
\vdots
\end{array}\right)\left(\begin{array}{lllll}
\ldots & u^{(i-1)} & u^{(i)} & u^{(i+1)} & \ldots
\end{array}\right)
\end{aligned}
$$

The diagonal elements of the matrix

$$
P E_{P P}=-\frac{3}{4}\left[\kappa_{(i-1,1)}^{2 / 3} u^{(i-1)} u^{(i)}-\left(\kappa_{(i-1, i)}^{2 / 3}+\kappa_{(i, i+1)}^{2 / 3}\right)\left\{u^{(i)}\right\}^{2}+\kappa_{(i, i+1)}^{2 / 3} u^{(i+1)} u^{(i)}\right]
$$

which is the same as Eq. (6.57). Similar to KE matrix, the non-diagonal elements of PE give the spatial displacement correlation of the elements with other elements in the system. 



\section{Chapter 7}

\section{Overview on continuum modeling of granular materials}

In the book of life every page has two sides: we human beings fill the upper side with our plans, hopes and wishes, but providence writes on the other side, and what it ordains is seldom our goal.

Nizami Ganjavi

The interest in the prediction of granular material behaviour in industrial applications has increased during the last decades. A wide variety of granular matter is employed, characterized by different dimensions and shapes of the grains, by their state of saturation or their temperature. In many applications the behaviour of granular materials is still unpredictable. For deeper insight, particles simulations can be used. A way to increase the capability and value of our simulations is to define a methodology able to bridge the microscale with macro-scale, which will give us an avenue in which we can capture numerically higher length and time scales. The proposed methodology will have the main feature of linking the properties of the grain scale, with those of the bulk, which could be accomplished by defining new constitutive laws or new contact models, based on micro-scale simulations and experiments. In the previous deliverables the appropriate procedures that would be used to obtain such methodology have been demonstrated depending on case studies of several researchers. The current report describes the numerical 
implementation of the proposed methodology with a focus on numerical simulations with a micro-macro transition, post processing analysis and visualization of micro- and macro-data. Finally, results of the numerical analyses are matched with theoretical predictions, which can give helpful insights in the definition of a continuum model and calibration. ${ }^{1}$

\subsection{Introduction}

With the development of computational power in recent years, the discrete particle/element method has gained its focus to the simulation community. However, this method has its own limitations in applying to the real world. One part is that the number of particle can be simulated is limited, normally in the order between $10^{4}$ to $10^{7}$ in a 3D setup, where as one normally has more than $10^{9}$ to $10^{11}$ particles in a real system. Another point is that the complexity of describing the real contact mechanics between particle in a numerical world, one has to make several assumptions to reduce this complexity to be able to simulate many particle contacts. Nevertheless, the DEM/DPM method is a really helpful tool for understanding the granules bulk behaviors qualitatively (and quantitatively for small systems) and thus one can explore the physics behind the scene for discrete nature of particulate systems where the traditional continuum solid/fluid mechanics can not explain.

An ambitious challenge in the granular material field is the accurate characterization of granular flow and its micromechanics under static and dynamic conditions. Granular flow is determined, on one hand, by the microscopic properties of the particles (e.g. friction, shape, morphology) and on the other hand, by the bulk /macroscopic properties of the bed of powder which is affected by the constraints exercised by the process equipment.

Despite numerous efforts, the constitutive relations describing the granular flow behavior are still a matter of debate. Solid mechanics and kinetic theory have been successful in predicting the solid and gas like behavior, respectively [71, 176]. At one extreme particles interact via enduring contacts, while in the gaseous regime, binary collisions are the mode of momentum exchange. The recently proposed inertial number framework has been successful in describing the flow behavior in the dense flow liquid like regime when the particles not only undergo collisions but also frictional interactions with other particles [11,

\footnotetext{
${ }^{1}$ In preparation: Taghizadeh, K., Iaconeta, I., Larese, A., Magnanimo, V., \& Luding, S. Journal of Applied Mechanics.
} 
$57,102,167]$. Though it very well predicts the flow behavior in case of steady shear of rigid particles, it fails in cases of inhomogeneous shear and transient shear, where we need better constitutive models to describe dense granular flow [200]. With the development of binning or coarse-graining methods, one can now obtain discrete simulation data with sufficient statistics that allow to derive constitutive relations that describe the local rheology and flow behavior [144, $152,204,227,258]$.

\subsection{Overview on continuum modeling}

Continuum mechanics is considered as a mathematical approach to solve engineering problems. Regarding matter as indefinitely divisible and locally homogeneous are the principle assumptions of the continuum theory. Accordingly, infinitesimal volume of material is noted as a particle in the continuum and there are neighbouring particles in all neighbourhood of a particle [129]. The most important continuum mechanical state variables are:

- Stress: expressing the effect of external loads ( the relation between loads and stresses is given by the equilibrium equation of continuum mechanics, for example the Cauchy equations in the simplest case).

- Strain: reflecting deformations (the relation between strains and deformations is given by geometrical equations).

Stress and strain are related to each other through constitutive equations, which expect to contain all the necessary information about the mechanical characteristics of the material.

Continuum models, which are raised upon assumptions of continuum mechanics, provide a reliable interpretation of material behaviour under various loading conditions. Since response of real material changes with regarding to amount of loading, there are many mathematical models, sometimes referred as constitutive equations, to characterize them. Constitutive equations are used in almost every engineering field such as: civil, chemical, mechanical, pharmaceutical engineering. As an example, in civil engineering practices, soil beneath the foundation plays an important role in the stability of the structures. It is considered as a complex material due to its unpredictive behaviour, thus, it needs to be fully characterized. In this respect, many constitutive models such as: Mohr-Coulomb, Cam Clay, Duncan-Change (hyperbolic), Plaxis Hardening, 
Hyperelastic, Hypoelastic models are developed and there will be fundamental improvements in future [128].

To find proper constitutive equations for granular assembly is not simple. Two approaches are followed by the majority of researchers: a continuummechanical and a micro-structural approach [15].

The idea of the continuum mechanical approach is to consider the assembly as a continuous domain, accept the concept of an infinitesimally small representative volume element (RVE), where stress and strain are the fundamental variables that uniquely determine the state of the material in any point. The parameters in the equations, expressing the specific properties of the material, are measured experimentally. This aspect makes this approach unreliable if the conditions, under which the experimental results are obtained, can not be replied.

Whereas the aim of the microstructural approach is to find macro-level state variables that are based on microvariables such as contact forces, grain displacements and local geometrical characteristics using suitable averaging techniques. Since the mechanical behaviour of granular material is essentially settled at the grain scale, a microstructural theory is expected to be far more reliable and general than the existing continuum-mechanical models.

It is evident that Discrete Element Method (DEM) has a direct access to the fundamental scale of granular materials and, as it becomes more accurate and computational power, it will allow for accurate representation of granular materials from their most basic granular scale. Unfortunately, nowadays, DEM models suffer from two major shortcomings: high computational cost and, related to this, inability to capture grain shape accurately. Use of smooth particles such as spheres and ellipsoids and enlargement of particles renders the model as just another phenomenological method [249]. Consequently, combine the strengths of the FEM and DEM methods by means of a multi-scale approach capturing the main features of the material becomes essential.

Recently, integration of modelling methods is the subject of the studies in various engineering disciplines. The reason for increasing interest in application of multi-scale approaches is the ability of these methods in interpreting and characterizing complex phenomena in materials. Multi-scale methods have emerged lately in mechanics to bridge different material scales ranging from atomic scale to continuum scale. These methods aim at obtaining constitutive responses at the continuum scale, without resorting to phenomenology, broadening the field of application.

The mechanical state of the assembly and its state-changing can exactly be described and predicted if the following characteristics are fully given: 
- position and geometry of each grain

- displacements (translation and rotation) of each grain

- contact forces

- material properties of the individual grains

A central problem in the micro-structural approach is the geometrical representation. First of all the geometrical unit has to be defined. Among of those used in literature for the theoretical description we can find: the grain itself, the contact between two particles, branch vectors or more complex unit as microelement, consisting of a grain and its neighbours.

The best-known mathematical representations applied for modelling whole systems of grains are the Voronoi tesselation and Delaunay network, where a clear description can be found in [15].

As a beginning, stress and strain tensors must be defined in terms of microvariables. Several micro-structural definitions have been presented until now.

Regarding the definition of stress tensor, [49] suggests the following average stress:

$$
\bar{\sigma}_{i j}=\frac{1}{V} \sum_{k=1}^{m} x_{i}^{k} T_{j}^{k}
$$

where $V$ is the spherical volume consisting of grains having arbitrary shape, submitted to the external force $T_{i}^{1}, \ldots, T_{i}^{m}$ on its boundary points $x_{i}^{1}, \ldots, x_{i}^{m}$.

[32] proposed the definition of the average stress with the help of contact forces inside the assembly of particle of arbitrary shape which, by means of the principal of virtual work, can be expressed as:

$$
\bar{\sigma}_{i j}^{\text {load }}=\frac{1}{V} \sum_{c=1}^{M} l_{i}^{c} F_{j}^{c}
$$

where $l_{i}^{c}$ is the branch vector forming the c-th contact and $F_{j}^{c}$ the c-th contact force arising between the grains.

[203] by means of the analysis of the grains' equilibrium obtains an equation similar to the previous one. 
Regarding the definition of strain, the average strain tensor has the following general formulation:

$$
\bar{\epsilon}_{i j}=\frac{1}{V} \sum_{e} \delta_{i}^{e} d_{j}^{e}
$$

where $\delta_{i}^{e}$ is the relative displacement and $d_{j}^{e}$ has been defined in several ways. In [117] and in [119] $d_{j}^{e}$ is the called polygon vector $h^{R S}$, defined as the vector obtained by counter-clockwise over $90^{\circ}$ of the rotated polygon vector $g^{R S}$ that connects the centres of adjacent polygons. A graphic description is given by Figure 7.1 :

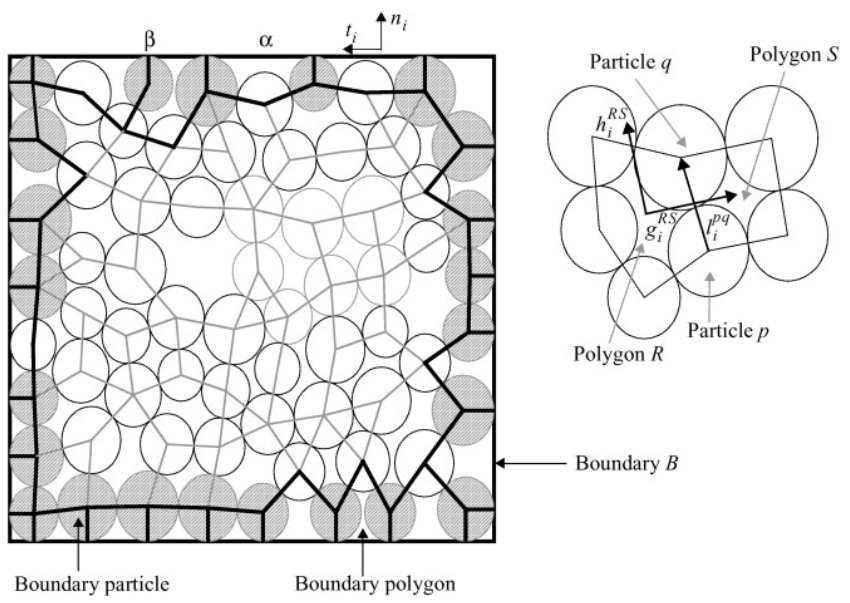

Figure 7.1: Polygons,branch vectors, polygon vector and rotated vector [113]

In [15] $d_{j}^{e}$ is the called complementary area vector, fundamental geometrical micro-variable of the system defined through a Delauney tesselation of the assembly.

In [15] the idea to use two dual cell systems, a material and spaced one respectively, is presented. The topological structure of the graphs is expressed by two topological matrices, which are employed to define the average stress and strain tensors and in the formulation of equilibrium and compatibility equations. The method of Bagi was formulated to 3D by, and used to analyze the strain and micro-parameters under tri-axial loading [50]. 
In [119] a micro-mechanical study is carried out. Since a packing of real heterogeneous granular materials is considered, the mechanical behaviour is expected to be heterogeneous, as well, exhibiting random variations. This implies the usefulness of statistical approach. Discrete element simulations have been performed to obtain the necessary data at the microscopic level, which would be very hard extract from experiments (photoelastic experiments). For each particle informations as displacements and forces are computed. Statistics, such as mean, standard deviations and probability distribution function were evaluated.

In [10] a more powerful model that rely on physics rather than phenomenology for granular matter is presented. The case of an elastoplastic material subjected to a triaxial compression is considered. The complex material behaviour is caught by extracting the evolution of those properties considered to fully describe the plasticity model, directly from the grain-scale mechanics. In this study the frictional resistance and the plastic dilatancy are individuated as the fundamental plastic variable. A hierarchical multi-scale approach is used to extract micro-mechanical state (stress and strain average tensor) and define the dependence of the fundamental plastic variables on the deformation. This study is applied in two different ways. The first one consists in performing discrete element simulations and linking them to finite element computations. The second one, performing a triaxial compression experiment, reading the necessary data by means of a tomography (technique) and a digital image correlation and linking them to finite element computations. In Figure 7.2 one can observe the schematic procedure of the multi-scale approach used in this study.

In computational mechanics some examples, where a combination between Finite Element Method and Discrete Element Method are present in literature, as well. Finite element method (FEM), as a continuum numerical analysis tools, is preferred among researchers due to its capability in modelling complex geometries and diverse boundary conditions provided for macroscopic models. A crucial aspect for the precise estimation of material response through multiscale approach is the ability to achieve continuum behavior directly from the particle-scale. Thus, FEM can be coupled with particle-scale methods such as discrete element method (DEM) [40]. Figure 7.3 illustrates a coupled FEM-DEM solution.

Yan et al. proposed coupling DEM to FEM domains to decrease numerical analyses cost through a layer of "ghost" particles located on surface FE facets, which deform by FEM domain [265]. In their study, the mesh generated by FEM does not cover the whole domain, instead the DEM and FEM overlap in a single layer of particles (i.e. ghost particles), see Figure 7.4. The algorithm in this 


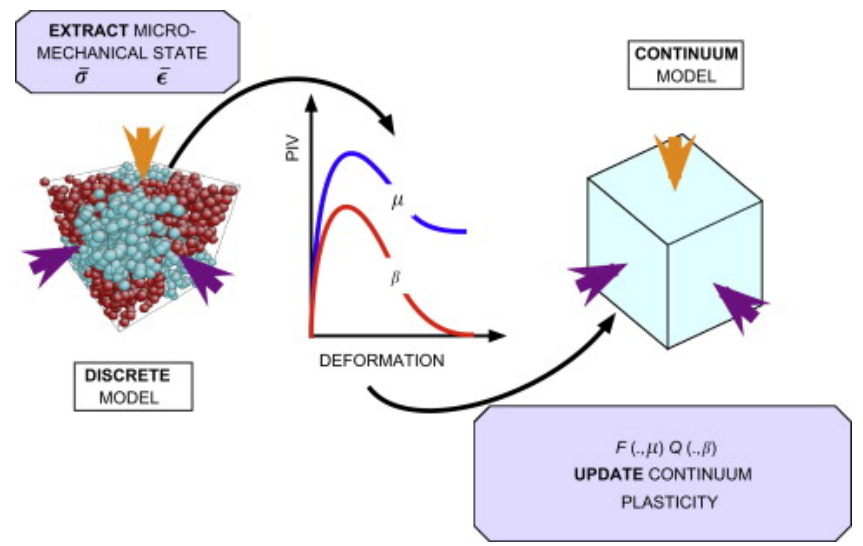

Figure 7.2: Schematic procedure of the hierarchical multiscale approach adopted [10]

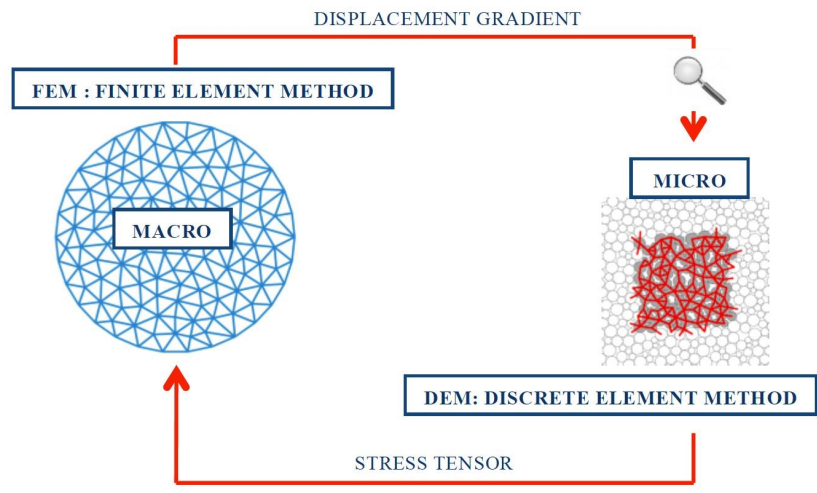

Figure 7.3: Coupling of FEM-DEM in simulation of cohesive granular material [179]

study includes a simultaneous exchange of data between DEM and FEM; free particles in the DEM simulation contribute to the boundary force in the FEM domain through ghost particles. On the other hand, the FEM domain produces the necessary data that allows the computation of the boundary condition on the particles involved in DEM through ghost particles as well. The particle-scale and continuum-scales function at the same time and exchange needed information dynamically. The results from simulation of pile penetration suggested that the 
artificial boundary effect can be alleviated by using the coupled FE facets with tuned FE continuum elastic compliance [265].

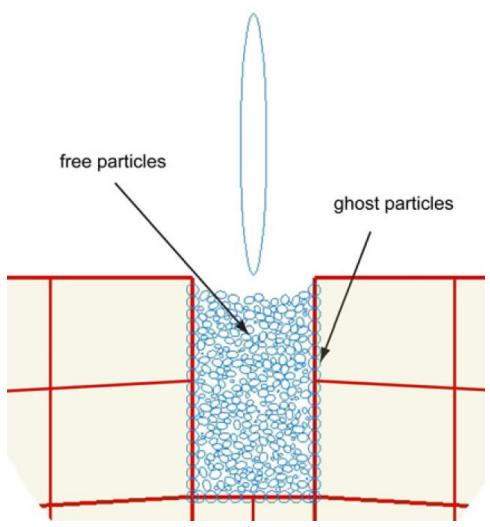

Figure 7.4: Schematic presentation of coupling between particle and continuum scale [265]

Guo and Zhao suggested a hierarchical multi-scale framework by coupling FEM and DEM for the simulation of cohesionless granular media [78]. In the framework, the DEM is used to characterize the highly nonlinear and disperse behaviour of granular assembly, while the continuum-based FEM is used to solve large-scale boundary value problems (BVPs). DEM assembly is attached to each Gauss integration point of the FEM mesh to perform as a representative volume element (RVE), through which the DEM and FEM exchange information such as stress, strain and tangent modulus. The hierarchical approach provides efficiency of FEM in solving large-scale BVPs. It also improves the conventional phenomenological assumptions on constitutive relation in continuum modelling [78]. The schematic illustration of hierarchical multi-scale modelling approach is presented in Figure 7.5.

Lu et al. developed a hybrid model of FEM and DEM capable of simulating the flow velocities and stresses of a bulk solid material stored in silos [140]. The FEM was employed to simulate the particles in the system as a continuum. Meanwhile, DEM was preferred in areas that the particle assembly no longer response as continuum, such as: intense shearing zones near intersection of silo walls and hopper section and the region near the outlet of the silo. The time steps selected in the FEM analysis are approximately 100 times larger than 


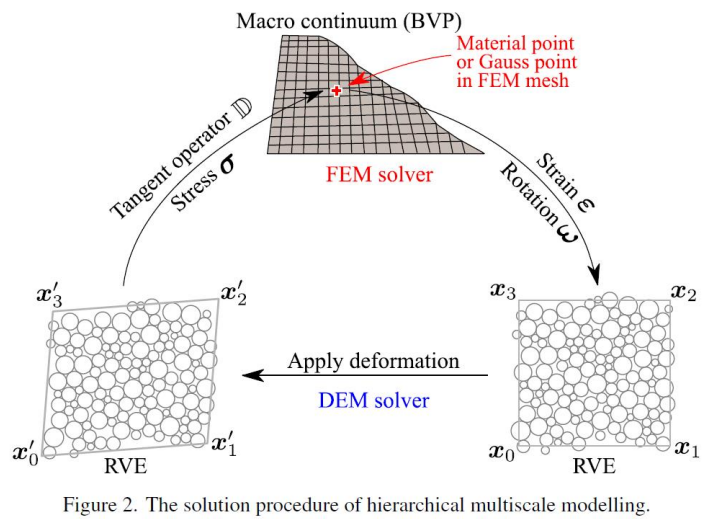

Figure 7.5: The solution procedure of hierarchical multi-scale modelling [78]

the DEM domain for calculation of velocity profiles and stress distributions. Furthermore, they conducted laboratory experiments involving gravity flow of soybean from a parallel wall bin for different outlet widths. As shown in Figure 7.6, good agreement was found between the wall pressures measured in the model silo and calculations from the numerical model based on finite-element and discrete element methods [140] [177].

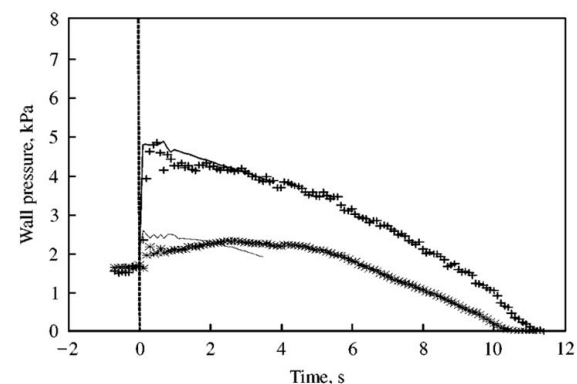

(a)

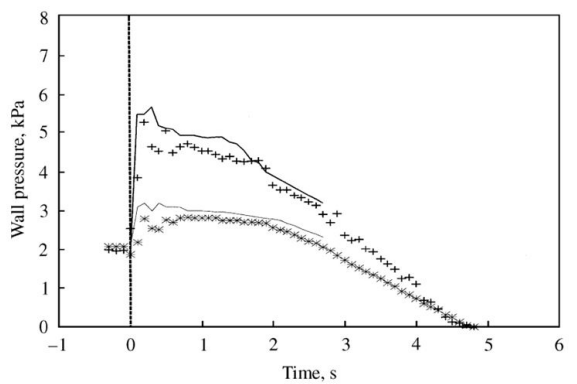

(b)

Figure 7.6: Comparison of numerical and experimental results of wall pressure $(+)$ Exp lower; (*) Exp upper; (-) DEM lower; (-) DEM upper a)outlet with of $50 \mathrm{~mm}$ b)outlet with of $90 \mathrm{~mm}[177]$ 


\subsubsection{Continuum model: a particle-based hypoelastic law}

A particle-based constitutive law, defined only for the elastic regime, is presented. The elastic response is formulated in the Eulerian description by a hypoelastic constitutive equation and by the elastic parameters of Bulk and Shear moduli expressed as functions of the volume fraction $v$. The theory behind the definition of the laws $K(v)$ and $\mu(v)$ was shown earlier in chapter.3. The constitutive law is implemented in a Material Point Method (MPM) code, which is based on the Finite Element theory and developed within the Kratos Multiphysics open source platform [43, 44].

The software is written for solving large displacement and large deformation problems; for this reason a distinction between reference and current configuration is made, considering the latter one for the resolution of the solving system. Furthermore, strain terms of higher order are taken into account, allowing a more accurate evaluation of the material response in non linear regime.

For a better understanding of the non linear finite element theory, used for the implementation of the software the authors suggest to refer to [262] for general purposes and to [89] for a detailed explanation of the formulation and algorithm of the method applied in this case.

In the definition of a rate constitutive law, the main concern, from a computational point of view, consists in defining a numerical integration of the constitutive model so that the resulting discrete equations satisfy the principle of material from indifference. In order to preclude the generation of spurious stresses in rigid body motions the following idea is adopted. Conceptually, the spatial, rate-constitutive equations are mapped to a local configuration which is unaffected by superposed rigid body motions. Then, a time-stepping algorithm is performed in this configuration, and the discrete equations are mapped back to the Eulerian configuration. In this work the methodology of Convective representation, which is essentially based on providing an algorithmic approximation of the rate of deformation tensor using the generalized mid-point rule, is adopted.

The algorithmic approximation of spatial, rate-like objects, such as, the rate of deformation tensor $\boldsymbol{d}$ and the Lie derivative of the Kirchhoff stress tensor $L_{v} \boldsymbol{\tau}$, in terms of incremental displacement $\boldsymbol{u}\left(\boldsymbol{x}_{n}\right)$ and the time increment $\Delta t$ is derived.

To obtain the approximation of $\boldsymbol{d}$ in convective description the following identity is exploited,

$$
\dot{\boldsymbol{C}}=2 \boldsymbol{F}^{T} \boldsymbol{d} \boldsymbol{F}
$$


where $\dot{C}$ is the time derivative of the right Cauchy-Green tensor and $\boldsymbol{F}$ is the total deformation gradient $\boldsymbol{F}=\frac{\partial \varphi}{\partial \boldsymbol{X}}$.

It is possible to rewrite the rate of deformation tensor as:

$$
d_{n+\alpha}=\frac{1}{2 \Delta t} f_{n+\alpha}^{-T}\left[f_{n+1}^{T} f_{n+1}-1\right] f_{n+\alpha}^{-1}
$$

where $\boldsymbol{f}_{n+\alpha}=\boldsymbol{F}_{n+\alpha} \boldsymbol{F}_{n}^{-1}$ and $\boldsymbol{f}_{n+1}=\boldsymbol{F}_{n+1} \boldsymbol{F}_{n}^{-1}$ are the deformation gradients relative to the configurations $\varphi_{n+\alpha}$ and $\varphi_{n+1}$, respectively.

Regarding $L_{v} \tau$, the algorithmic approximation is obtained exploiting the following identity

$$
L_{\nu} \boldsymbol{\tau}=\boldsymbol{F} \dot{\boldsymbol{S}} \boldsymbol{F}^{T}
$$

where $\dot{S}$ is the time derivative of the Second Piola-Kirchhoff stress tensor. The approximation of $L_{v} \tau$ in convective description is:

$$
L_{\nu} \tau_{n+\alpha}=\frac{1}{\Delta t} f_{n+\alpha}\left[f_{n+1}^{-1} \tau_{n+1} f_{n+1}^{-T}-\tau_{n}\right] f_{n+\alpha}^{T}
$$

Assuming a rate constitutive equation of the form

$$
L_{\nu} \tau=\boldsymbol{a}: \boldsymbol{d}
$$

with $\boldsymbol{a}$ representing the tensor of elastic moduli, and replacing the expressions of Equations 7.5 and 7.7 in Equation 7.8, the updated formula is

$$
\tau_{n+1}=f_{n+1} \tau_{n} f_{n+1}^{T}+a_{n+1}: \frac{1}{2}\left[1-f_{n+1}^{-T} f_{n+1}^{-1}\right]
$$

The algorithms for the elastic response and the evaluation of of $K^{n+1}$ and $\mu^{n+1}$ are described in Tables 7.1 and 7.2.

In the following an example of isotropic compression is presented.

The validation study is performed by using as material parameters of input the data listed in Table 7.3.

The analysis is performed by using a quasi-static time scheme with prescribed displacement imposed along each of three directions, with a value such that the resulting strain rate for each time step is equal to -0.0001 . The analysis ends when the volume fraction reaches a value of 0.82 .

In the following the numerical results obtained with the particle-based hypoelastic law are presented in terms of coordination number $C^{*}$ and pressure $P$ as functions of the cumulative strains. 
STEP 1: Evaluate the relative deformation gradient

$\boldsymbol{f}_{n+1}=\boldsymbol{F}_{n+1} \boldsymbol{F}_{n}^{-1}$

STEP 2: Evaluate the left Cauchy-Green tensor and Almansi strain tensor $\boldsymbol{b}_{n+1}=\boldsymbol{f}_{n+1} \boldsymbol{f}_{n+1}^{T}$

$\boldsymbol{e}_{n+1}=\frac{1}{2}\left[\mathbf{1}-\boldsymbol{b}_{n+1}^{-1}\right]$

STEP 3: Evaluate the Bulk and Shear moduli and $\boldsymbol{a}$ as function

of the volume fraction (see Table 7.2)

$K=K(v), G=G(v), \boldsymbol{a}=\boldsymbol{a}(v)$

STEP 4: Evaluate the stress rate tensor

$L_{v} \tau_{n+1} \Delta t=\boldsymbol{a}(v): \boldsymbol{e}_{n+1}$

STEP 5: Update the stress tensor

$\tau_{n+1}=f_{n+1} \tau_{n} f_{n+1}^{T}+L_{\nu} \tau_{n+1} \Delta t$

Table 7.1: Algorithm of the hypoelastic constitutive law

Input parameters: $J, v_{0}, C_{0}, C_{1}, \alpha, g_{3}, F_{\nu 0}, K_{0}, K_{1}, G_{0}, G_{1}, G_{2}$

STEP 1: Update the volume fraction

$v_{n+1}=v_{0} \cdot e^{-(J-1)}$

STEP 2: Evaluate the coordination number

$C^{*}=C_{0}+C_{1}\left(\frac{v}{v_{0}}-1\right)^{\alpha}$

STEP 3: Evaluate the trace of fabric

$F_{\nu}=C^{*}(v) \cdot g_{3} \cdot v$

STEP 4: Evaluate the bulk and shear moduli

$K=K_{0}+K_{1}\left(F_{\nu}-F_{\nu 0}\right) ; \quad G=G_{0}+G_{1}\left(F_{\nu}-F_{\nu 0}\right)^{G_{2}}$

Table 7.2: Algorithm for the computation of the bulk and shear modulus

\begin{tabular}{lllllllllll}
\hline$v_{0}$ & $C_{0}$ & $C_{1}$ & $\alpha$ & $g_{3}$ & $F_{0}^{0}$ & $K_{0}$ & $K_{1}$ & $G_{0}$ & $G_{1}$ & $G_{2}$ \\
\hline \hline 0.665 & 4 & 9.5 & 0.375 & 1.24 & 3.63 & 16005 & 4350 & 13700 & 5083.5 & 0.723653 \\
\hline
\end{tabular}

Table 7.3: Isotropic compression: input parameters in particle-based hypoelastic law

For the verification of the constitutive law the values of the Bulk and Shear moduli evaluated numerically and analytically are compared. From Figures 7.9 and 7.10 it is possible to observe that the values coincide. As future work, a comparison of the numerical results in terms of pressure evaluated through the 
particle-based constitutive law and a Discrete Element Method code has to be performed.

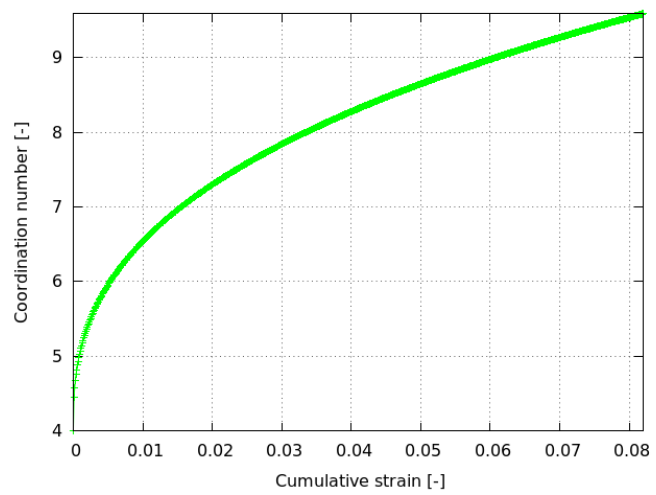

Figure 7.7: Isotropic compression: coordination number.

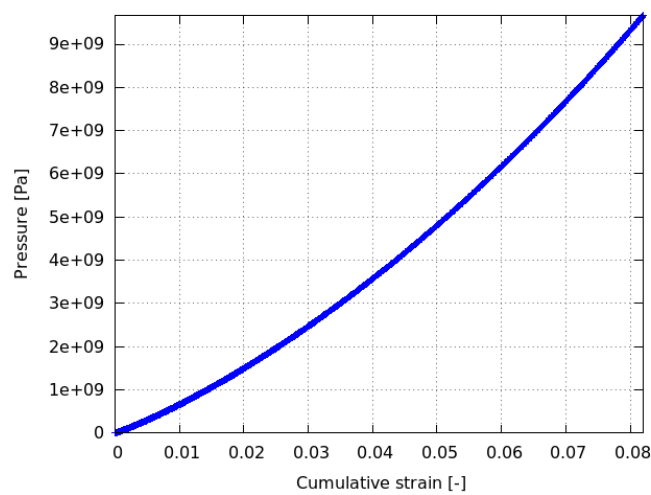

Figure 7.8: Isotropic compression: pressure. 


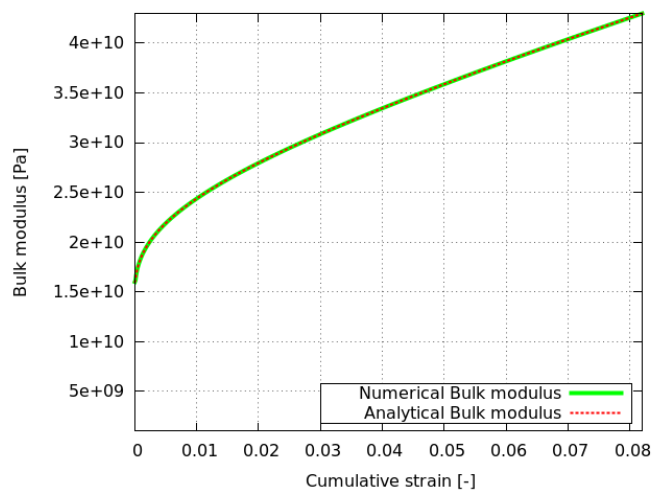

Figure 7.9: Isotropic compression: comparison of Bulk modulus evaluated numerically and analytically.

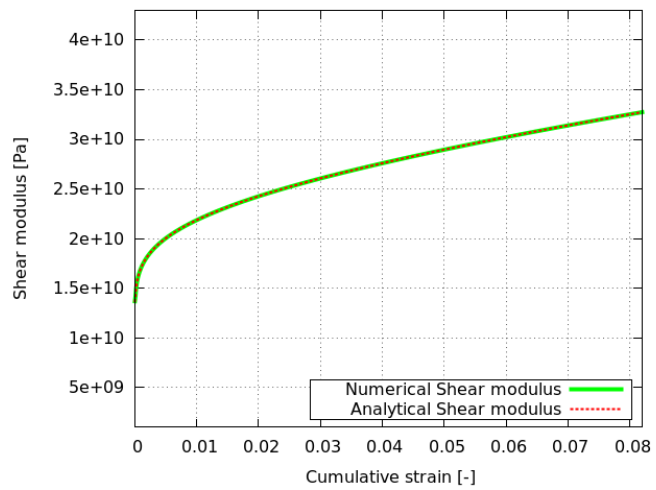

Figure 7.10: Isotropic compression: comparison of Shear modulus evaluated numerically and analytically.

\subsection{Conclusion}

This chapter showed the philosophy behind micro-macro transition, data analysis and continuum modeling cases where modern, advanced data analysis has been applied and critically tested. Many different microscopic quantities can be extracted by DEM simulations, like position, velocity, number and orienta- 
tion of contacts, forces for each individual particle in the numerical sample, as functions of space and time. We described this using different examples. Nevertheless the application of DEM is constrained by its computational cost to small systems, i.e. when a process involving few (less than a million) particles or a (local) representative element of the full system is considered. Therefore, the wide set of information has to be transferred into macroscopic field variables in order to make them usable for industrial and technological applications. Only a multi-scale/multiphase continuum analysis can fully solve the challenges that await those who want to understand and improve realistic particulate systems of industrial relevance. It is possible to link the macro-scale with the insights obtained at micro-scale, by proper averaging and coarse-graining procedures, and solve the micro-macro gap by means of a multi-scale approach coupling discrete and continuum techniques. This is a fundamental feature of granular materials as the behaviour at macro-scale is strongly related to smaller-scale field variables and kinetic processes. Continuum fields can be obtained by the novel coarse-graining methods development in T-MAPPP, as well as by suitable post-processing and on-the-fly data-analysis tools, able to deal with the enormous amount of data resulting from DEM. Interpretation and increased understanding can be reached by averaging in space and time with various different resolutions and a rich output result from data mining. 


\section{Chapter 8 \\ Conclusion and Outlook}

\section{Conclusion}

Dense granular media are of widespread importance in a number of applications, ranging across time and length scales from geophysical earthquakes in the San Andreas fault zone, to plastic powder sintering in the 3D printer on your desk. The granular processes involved can be very slow, almost static, or very fast, highly dynamic. Such particle-based materials, like granular soils, are different from 'classical' materials such as crystals or watery liquids; they are neither fluid- nor solid-like, but often display both behaviors at the same time. This solid-fluid phase behavior requires precise predictive modeling tools, while this complex phase behavior also poses fundamental questions as to how it emerges as collective effect. Understanding the response of granular-based systems in applications requires detailed grasp of the connection between the basic ingredients (particles) and the macroscale properties of the systems considered. These are complex systems and an understanding of the overall behaviour cannot be gained by studying individual particles. While significant progress has been made during the last decades on understanding relevant physical mechanisms, there is still many open questions, starting from the physics of particle interactions to general features of multiscale models that will bridge the different spatial and temporal scales of interest.

These days, it has been well understood that grain-scale properties control the bulk-scale behavior of the granular material. The aim of this thesis was to study and determine the micromechanical mechanisms that govern various phenomena that are challenging in the world of particles. This leads to a better 
understanding of complexities in macro-scale response of particulate systems. Several noteworthy conclusions can be drawn from the research and are listed per chapter below.

Numerical simulations, e.g. Discrete Element Method (DEM) on the particle scale or the Finite Element Method (FEM) on the continuum scale, have revealed the utmost role of the microstructure in characterising the elastic behaviour of granular soils. Especially, DEM is a powerful tool to inspect the influence of the microscopic contact properties of the individual constituents on the bulk behavior of granular assemblies. In the first part of this thesis, isotropic and deviatoric deformations are applied to polydisperse packings of frictional and cohesive spheres; after preparation by isotropic compression of samples with different inter-particle contact friction and adhesion, at various volume fraction, the effective elastic moduli is determined from the incremental stress response to the application of strain-probes (chapter 2). Then, numerical results were compared with analytical formulations for the bulk and the shear moduli and observations were discussed in chapter 3 . The main observations of these chapters are drawn as:

- Chapter 2: At first, simulation results using the Discrete Element Method (DEM) of frictional, cohesive, disordered packings of dense granular materials are presented. The goal is to gain a better understanding of the mechanical behavior of granular matter. Afterwards, the micro- and macromechanical behavior of similar assemblies emphasizing the effect of interparticle friction and cohesion is analyzed on macro-mechanical parameters, e.g. pressure, energy, and inertial number. A particular attention is devoted to the effect of inter-particle micro-parameters on the elastic and plastic response of the assemblies. It was shown that the effect of interparticle parameters are not negligible, where friction between particles enhances macroscopic results more than cohesion. Thus, selecting a right set of input parameters is an essential task to achieve reliable outputs.

- Chapter 3: Next, the prepared assemblies of polydisperse, linearly elastic frictional spheres are used to compare the data from DEM simulations with predictions from well-established micromechanical models, namely the Effective Medium Theory (EMT) and the Fluctuation Theory (FT). Both theories do not account for the effect of different preparation history (different inter-particle friction coefficients) on the elastic moduli. The fluctuation theory is in agreement with numerical data, almost perfect for the bulk modulus and close for the shear modulus, at least in the intermediate compression regime, but does not capture the anomalous behavior 
where the theory overpredicts. This analysis remands to the open question on what statistical measures are needed for a comprehensive description of the microstructure. For dense systems, the coordination number (and its local fluctuations) seems to be a sufficient measure of the contact geometry. Simulations and theory still diverge for poorly-coordinated samples, when the jamming point is approached, i.e. the transition from solid to fluid state.

Most soils are mixtures of two or more components; the relative amount of species affect the overall behavior, but the multi-scale mechanisms behind this behavior are far from understood. Chapter 4 was devoted to the effect of the mixtures composition on the bulk elastic stiffness and attenuation using a well-established wave propagation technique. Along with the experiments, the chapter gives a comparison between simulations and experiments. The objective of chapter 5 was to improve the simulation results of mixture assemblies by introducing a new contact approach. Below, the main outcomes of chapter 4 and 5 are depicted as:

- Chapter 4: The elastic response of disordered mixtures of granular and soft matter is investigated by means of wave-propagation, both experimentally and numerically. This allows inferring fundamental properties of granular and soft disordered materials such as elastic moduli and dissipation mechanisms. Mixtures are prepared with different volumes of soft matter mixed with hard matter to identify the transition from a rigid to a soft granular skeleton. Interestingly, we find that the behavior is highly non-linear and also non-monotonic with increasing the percentage of soft content. It was found that the stiffness in a granular mixture increases if soft inclusions are added in proper amount. In short, the main goal of this chapter was to enhance the dissipative, elastic and lightweight properties of materials (like soils, asphalt, etc.) by deliberately adding dissipative, soft, light inclusions of various types and compositions. This allows for a novel design methodology for calm, smooth, and smart materials that can be better in various aspects than their separate components. Particularly, the chapter aimed at making improved materials like soils.

- Chapter 5: Although, the qualitative agreement between physical experiment and simulations was well captured, complex interactions between soft and stiff particles yet renders several ongoing challenges like, e.g., the presence multi-body-interactions instead of pairwise contacts. Hence, 
the emerge of right interaction law between soft particles leads to introduce stress-based non-binary contact model, missing in classical discrete element methods (DEM), and compare to the classical DEM and strainbased non-binary contact model for highly deformed spheres; which takes into account not only the non-binary interaction of particles but also the influence of neighboring particles. It was shown that the new proposed contact model provides a better agreement between simulations and experiments; furthermore, the new proposed contact model is quite faster than the previous versions of multi-contact models.

Numerical simulations, based on the Discrete Element Method, have revealed the essential role of the microstructure in characterising the elastic behaviour of granular soils. However, continuum models are more desirable for applications, where the macroscopic field variables can be obtained via micromacro averaging procedures on small, representative elementary volumes. In the final stage of this thesis, the attempt is to develop a macroscopic continuum model for the granular materials, based on micro-scale information obtained by the DEM simulations; by involving not only the elastic moduli and the relation between effective moduli and microstructure but also the attenuation and dispersion behavior for all relevant frequencies. The main outputs of chapter 6 and 7 are concluded as:

- Chapter 6: From experiments, it was found that adding soft rubber inclusions (i.e. disorder) reduces unwanted vibrations and noise generated for higher damping. Thus, a stochastic-based theoretical model, involving a reduced complexity, for granular systems that predicts the influence of vibrations is applied; which, instead of dealing with the too many eigenmodes of the system, we propose an approach with reducsed complexity, where the frequencies are grouped in bands. The results for disordered granular chains showed that at high-frequency, energy dispersion and dissipation are stronger than at low-frequency, which produces amplitude attenuation, e.g. waveform distortion allows only for low-resolution seismic data analysis, especially for moderately to deeply buried formations.

- Chapter 7: Finally, chapter 7 gives an overview on implementation of micro-based constitutive laws in a Finite Element platform to simulate selected larger i.e. continuum-scale problems. 


\section{Outlook}

The work in this thesis is undertaken as a step forward towards understanding the relationship between microscale particle properties and macroscopic behavior, e.g. elastic stiffness of granular assemblies, from experimental, numerical and theoretical points of view. There are several limitations and many aspects that require further research. Thus, it is recommended that future studies address the following issues:

- The analysis of the interplay of micromechanical properties (particle size, visco-elastic moduli (stiffness), densities) and system properties (composition, stress state, anisotropy) on the sound absorption behavior of granular aggregates and mixtures is still an open field of research.

- Among diverse physical properties of individual particles in particulate materials, the shape and morphology play important roles in shear strength and flowability of the bulk. Additionally, it has been observed that the particle shape and the resulting geometric interlocking between particles is a primary contributor to the overall shearing resistance and is therefore critical for predicting the behaviour of a granular assembly. Hence, one need to study the effect of shape irregularities on the bulk response of granular packings.

- The majority of the research on granular materials carried out in the past have concentrated on single species (single property) particles. Relatively little effort was invested to design, e.g. lightweight material by using a mixture of different species with different material properties.

- There is very limited study about dissipation mechanisms in granular materials: numerical simulation at both particle and continuum level could give a better insight at the microscopic and effective macroscopic scales.

- A systematical experimental investigation aimed to reduce unwanted acoustic noise in granular mixtures, has still not yet been carried out.

- A predictive wave mode, e.g. based on the reduced order approach, has never been applied to granular materials, specifically in case of mixtures, to properly describe the energy transfer between frequencies associated to different particle species.

- To the best of our knowledge, there are still no contributions devoted to the analysis of acoustic absorption in soft-stiff mixtures, in particular 
aimed to the smart design of mixtures with tailored damping properties, e.g. in a pre-defined frequency band.

- Works devoted to the investigation of recyclable materials mixed with granular soils in order to reduce costs along with the environmental footprint is missing.

- To gain more insights from the wave propagation experiments, photoelastic disks (2 or 3D) and high speed cameras can be employed during to access the sound propagation properties and microstructural information of granular packings.

- Future work will focus on the extension of our small perturbation approach to elasto-plasticity and predicting the plastic response of a granular assembly under large deformation. 


\section{Bibliography}

[1] V Achilleos, G TheochaRis, and CH SKokos. "Energy transport in one-dimensional disordered granular solids". In: Physical Review E 93.(2) (2016), p. 022903 (cit. on p. 122).

[2] I Agnolin and NicolaAs P KrUYT. "On the elastic moduli of twodimensional assemblies of disks: Relevance and modeling of fluctuations in particle displacements and rotations". In: Computers \& Mathematics with Applications 55.(2) (2008), pp. 245-256 (cit. on p. 49).

[3] I. AGNolin and J.N. Roux. "Internal states of model isotropic granular packings. III. Elastic properties". In: Physical Review E 76.(6) (2007), p. 061304. DOI: 10.1103/PhysRevE. 76.061304 (cit. on p. 9).

[4] IVANa Agnolin and JeAn-NoËL Roux. "Internal states of model isotropic granular packings. III. Elastic properties". In: Physical Review E 76.(6) (2007), p. 061304 (cit. on pp. 3, 58, 72).

[5] K. AKI and P. RichaRds. Quantitative Seismology. Freeman, New York, 1980 (cit. on p. 10).

[6] KeIITI AKI and PAUL G Richards. Quantitative seismology. 2002 (cit. on p. 122).

[7] Philip B Allen and Jonathan Kelner. "Evolution of a vibrational wave packet on a disordered chain". In: American Journal of Physics 66.(6) (1998), pp. 497-506 (cit. on pp. 122, 130, 135). 
[8] F Alonso-Marroquin, S Luding, HJ HERRMANn, and I VARdoulakis. "Role of anisotropy in the elastoplastic response of a polygonal packing". In: Physical Review E 71.(5) (2005), p. 051304 (cit. on p. 49).

[9] G Alvarado and MattheW Richard Coop. "On the performance of bender elements in triaxial tests". In: Géotechnique 62.(1) (2012), p. 1 (cit. on p. 94).

[10] J.E. Andrade, C.F. Avila, S.A. Hall, N. Lenoir, and G. Viggiani. "Multiscale modeling and characterization of granular matter: from grain kinematics to continuum mechanics". In: Journal of the Mehcanics and Physics of Solids 59 (2011), pp. 237-250 (cit. on pp. 161, 162).

[11] Bruno Andreotti, YoËl Forterre, and Olivier Pouliquen. Granular media: between fluid and solid. Cambridge University Press, 2013 (cit. on p. 156).

[12] Mohsen Asadi, Ahmad Mahboubi, and Klaus Thoeni. "Discrete modeling of sand-tire mixture considering grain-scale deformability". In: Granular Matter 20.(2) (2018), p. 18 (cit. on p. 84).

[13] Mohsen Asadi, Klaus Thoeni, and Ahmad Mahboubi. "An experimental and numerical study on the compressive behavior of sandrubber particle mixtures". In: Computers and Geotechnics 104 (2018), pp. 185-195 (cit. on p. 84).

[14] JH AtKinson and G SALLfors. "Experimental determination of soil properties. General Report to Session 1". In: Proc. 10th ECSMFE, Florence. Vol. 3. 1991, pp. 915-956 (cit. on pp. 7, 8).

[15] K. BAGI. "Stress and strain in granular assemblies". In: Mechanics of Materials 22.(3) (1996), pp. 165-177 (cit. on pp. 158-160).

[16] JP BARDET. "Numerical simulations of the incremental responses of idealized granular materials". In: International Journal of Plasticity 10.(8) (1994), pp. 879-908 (cit. on p. 49).

[17] Danielle S Bassett, Eli T Owens, Karen E Daniels, and Mason A PORTER. "Influence of network topology on sound propagation in granular materials". In: Physical Review E 86.(4) (2012), p. 041306 (cit. on p. 82).

[18] RJ Bathurst and Leo Rothenburg. "Micromechanical aspects of isotropic granular assemblies with linear contact interactions". In: Journal of applied mechanics 55.(1) (1988), pp. 17-23 (cit. on p. 60). 
[19] R. Bellotti, M. Jamiolkowski, D.C.F. LoPresti, and D.A. O’Neill. "Anisotropy of small strain stiffness in Ticino sand". In: Géotechnique 46.(1) (1996), pp. 115-131 (cit. on p. 9).

[20] Peter J Bosscher, Tuncer B Edil, and Senro Kuraoka. "Design of highway embankments using tire chips". In: JGGE 123.(4) (1997), pp. 295-304 (cit. on pp. 3, 76).

[21] LEON BRILlOUIN. Wave propagation in periodic structures: electric filters and crystal lattices. Courier Corporation, 2003 (cit. on p. 122).

[22] Nicolas Brodu, Joshua A. DiJksman, and Robert P. Behringer. "Multiple-contact discrete-element model for simulating dense granular media". In: Physical Review E - Statistical, Nonlinear, and Soft Matter Physics 91.(3) (2015), pp. 1-6. DOI: 10.1103/PhysRevE. 91.032201 (cit. on pp. 99, 100, 103, 104, 106, 107, 111).

[23] Nicolas Brodu, Joshua A Dijksman, and Robert P Behringer. "Spanning the scales of granular materials through microscopic force imaging". In: Nature communications 6 (2015), p. 6361 (cit. on p. 106).

[24] JB BURLAND. "Small is beautiful'-the stiffness of soils at small strains: Ninth Laurits Bjerrum Memorial Lecture". In: Canadian Geotechnical Journal 26.(4) (1989), pp. 499-516 (cit. on pp. 7, 8).

[25] Ramon CABiscol, JAn Henrik Finke, and ARno Kwade. "Calibration and interpretation of DEM parameters for simulations of cylindrical tablets with multi-sphere approach". In: Powder Technology 327 (2018), pp. 232-245 (cit. on p. 98).

[26] MA Celigueta, S latorre, F Arrufat, and E OÑate. "Accurate modelling of the elastic behavior of a continuum with the Discrete Element Method". In: Computational Mechanics 60.(6) (2017), pp. 997-1010 (cit. on p. 100).

[27] Minsu Cha, Gye-Chun Cho, and J Carlos Santamarina. "Longwavelength P-wave and S-wave propagation in jointed rock masses". In: Geophysics 74.(5) (2009), E205-E214 (cit. on p. 76).

[28] Ching S CHANG, SAO J CHAO, and Y ChAng. "Estimates of elastic moduli for granular material with anisotropic random packing structure". In: International journal of solids and structures 32.(14) (1995), pp. 19892008 (cit. on pp. 49, 61). 
[29] Korchoke Chantawarangul. "Numerical simulations of threedimensional granular assemblies". In: Ph. D. Thesis, Dept. of Civil Engineering, Univ. of Waterloo 219 (1993) (cit. on p. 60).

[30] Y.-C. Chen, I. Ishibashi, and J.T. Jenkins. "Dynamic shear modulus and fabric: part I, depositional and induced anisotropy". In: Géotechnique 38.(1) (1988), pp. 25-32 (cit. on p. 9).

[31] Peng Cheng and Gary F Margrave. "Estimation of Q: a comparison of different computational methods". In: CSPG/CSEG/CWLS GeoConvention 12 (2013), pp. 2-5 (cit. on p. 91).

[32] J. Christoffersen, M.M. Mehrabadi, and S. Nemat-Nasser. "A micromechanical description of granular material behaviour". In: Journal of Applied Mechanics Transactions of the ASME 48 (1981), pp. 339-344 (cit. on p. 159).

[33] J Christoffersen, MM Mehrabadi, and Sia Nemat-Nasser. "A micromechanical description of granular material behavior". In: Journal of applied mechanics 48.(2) (1981), pp. 339-344 (cit. on pp. 3, 54).

[34] KLoss Christoph. Source code for MC-DEM simulations and simulation setup for hydrogel multicontact example. https : / github.com/ CFDEMproject/LIGGGHTS - PUBLIC. [Online; accessed 1Jan 17, 2017]. 2017 (cit. on p. 113).

[35] CRI ClaYTON. "Stiffness at small strain: research and practice". In: Géotechnique 61.(1) (2011), pp. 5-37 (cit. on pp. 8, 21, 49).

[36] Paul W Cleary and Mark L Sawley. "DEM modelling of industrial granular flows: 3D case studies and the effect of particle shape on hopper discharge". In: Applied Mathematical Modelling 26.(2) (2002), pp. 89-111 (cit. on p. 98).

[37] G COMBE and JN Roux. "Good practice for sample preparationConstruction of granular packings". In: ALERT Doctoral School 2017 Discrete Element Modeling (2017), p. 99 (cit. on pp. 48, 98).

[38] Christophe Coste and Bruno Gilles. "Sound propagation in a constrained lattice of beads: High-frequency behavior and dispersion relation". In: Physical Review E 77.(2) (2008), p. 021302 (cit. on p. 122).

[39] STEPHEN C Cowin. "The relationship between the elasticity tensor and the fabric tensor". In: Mechanics of Materials 4.(2) (1985), pp. 137-147 (cit. on pp. 3, 49). 
[40] P.A. Cundall and O.D.L. Strack. "A discrete numerical model for granular assemblies". In: Géotechnique 29.(1) (1979), pp. 47-65 (cit. on p. 161).

[41] Peter A Cundall and Otto DL Strack. "A discrete numerical model for granular assemblies". In: geotechnique 29.(1) (1979), pp. 47-65 (cit. on pp. $4,20,50,123)$.

[42] Peter A Cundall and Otto DL Strack. "A discrete numerical model for granular assemblies". In: Geotechnique 29.(1) (1979), pp. 47-65 (cit. on pp. 98, 100).

[43] P. DADVAND. A framework for developing finite element codes for multi-disciplinary applications. PhD thesis: Universidad Politécnica de Cataluña, 2007 (cit. on p. 165).

[44] P. Dadvand, R. Rossi, and E. OÑate. "An object-oriented environment for developing finite element codes for multi-disciplinary applications". In: Archives of Computational Methods in Engineering 17 (2010), pp. 253-297 (cit. on p. 165).

[45] Chiara Daraio, VF Nesterenko, EB Herbold, and Sungho Jin. "Energy trapping and shock disintegration in a composite granular medium". In: Physical Review Letters 96.(5) (2006), p. 058002 (cit. on p. 122).

[46] J. DAVID and N. CHEEKE. Fundamentals and Applications of Ultrasonic Waves. CRC Press, Boca Raton, 2002 (cit. on p. 11).

[47] PJ DigBy. "The effective elastic moduli of porous granular rocks". In: Journal of Applied Mechanics 48.(4) (1981), pp. 803-808 (cit. on pp. 9, 49, 59).

[48] MaKsym Dosta, Clara Costa, and Hazim Al-Qureshi. "Numerical investigation of compaction of deformable particles with bonded-particle model". In: EPJ Web of Conferences. Vol. 140. EDP Sciences. 2017, p. 15021 (cit. on p. 99).

[49] A. Drescher and G. Josselin De Jong. "Photoelastic verification of a mechanical model for the flow of a granular material". In: Journal of the Mechanics and Physics of Solids 20 (1972), pp. 337-351 (cit. on p. 159). 
[50] O DurÁn, N P KruYT, and S LUding. "Analysis of three-dimensional micro-mechanical strain formulations for granular materials: Evaluation of accuracy". In: International Journal of Solids and Structures 47.(2) (2010), pp. 251-260. DOI: 10.1016/j . ijsolstr. 2009.09.035 (cit. on p. 160).

[51] Danial Rezazadeh Eidgahee and Ehsan Seyedi Hosseininia. "Mechanical behavior modeling of sand-rubber chips mixtures using discrete element method (DEM)". In: AIP Conference Proceedings. Vol. 1542. 1. AIP. 2013, pp. 269-272 (cit. on p. 76).

[52] AMr S ELNASHAI and LUIGI Di SARNO. Fundamentals of earthquake engineering. Wiley New York, 2008 (cit. on p. 77).

[53] T MATthew Evans and Julio R VAldes. "The microstructure of particulate mixtures in one-dimensional compression: numerical studies". In: Granular Matter 13.(5) (2011), pp. 657-669 (cit. on pp. 3, 76, 85).

[54] A. Ezaoui and H. Di Benedetto. "Experimental measurements of the global anisotropic elastic behaviour of dry Hostun sand during triaxial tests, and effect of sample preparation". In: Géotechnique 59.(7) (2009), pp. 621-635 (cit. on p. 9).

[55] Abdoulaye Fall, Gulllaume Ovarlez, David Hautemayou, CÉdric MÉZIÈRE, J-N ROUX, and FrANÇOIS CHEVOIR. "Dry granular flows: Rheological measurements of the $\mu$ (I)-rheology". In: Journal of rheology 59.(4) (2015), pp. 1065-1080 (cit. on p. 35).

[56] Andrew T Fenley, Hari S Muddana, and Michael K Gilson. "Calculation and visualization of atomistic mechanical stresses in nanomaterials and biomolecules". In: PloS one 9.(12) (2014), e113119 (cit. on p. 105).

[57] Y Forterre and O Pouliquen. "Flows of Dense Granular Media". In: Annu. Rev. Fluid Mech. 40 (2008), pp. 1-24 (cit. on p. 157).

[58] Z. FOURNIER et al. "Mechanical properties of wet granular materials". English. In: Journal of Physics Condensed Matter 17.(9) (2005), S477S502 (cit. on p. 21).

[59] GÖRAN FRENNING. "An efficient finite/discrete element procedure for simulating compression of 3D particle assemblies". In: Computer Methods in Applied Mechanics and Engineering 197.(49-50) (2008), pp. 42664272 (cit. on p. 99). 
[60] GÖRAN FrenNing. "Towards a mechanistic model for the interaction between plastically deforming particles under confined conditions: A numerical and analytical analysis". In: Materials Letters 92 (2013), pp. 365-368 (cit. on p. 99).

[61] RicARdo DE Frias Lopez, JonAs EKBlad, and Johan SilfWERbrand. "Resilient properties of binary granular mixtures: a numerical investigation". In: Computers and Geotechnics 76 (2016), pp. 222-233 (cit. on p. 3).

[62] Francesco Froilo and J-N Roux. "Incremental response of a model granular material by stress probing with DEM simulations". In: AIP Conference Proceedings. Vol. 1227. 1. AIP. 2010, pp. 183-197 (cit. on p. 56).

[63] Georg C Ganzenmüller, Martin O Steinhauser, Paul Van LiedekERKE, and KATHOLIEKE UNIVERSTITEIT LEUVEN. "The implementation of Smooth Particle Hydrodynamics in LAMMPS." In: (2011) (cit. on p. 108).

[64] Vinod K GARga and Vince O'Shaughnessy. "Tire-reinforced earthfill. Part 1: Construction of a test fill, performance, and retaining wall design". In: Canadian Geotechnical Journal 37.(1) (2000), pp. 75-96 (cit. on pp. 3,76$)$.

[65] Johan Gaume, Guillaume Chambon, and Mohamed NaAim. "Quasistatic to inertial transition in granular materials and the role of fluctuations". In: Physical Review E 84.(5) (2011), p. 051304 (cit. on p. 35).

[66] Michel GÉRAdin and DANIEL J RiXEN. Mechanical vibrations: theory and application to structural dynamics. John Wiley \& Sons, 2014 (cit. on p. 131).

[67] David T Gethin, Roland W Lewis, and RAJEsh S RANSing. "A discrete deformable element approach for the compaction of powder systems". In: Modelling and simulation in Materials Science and Engineering 11.(1) (2002), p. 101 (cit. on p. 98).

[68] FA Gilabert, J-N Roux, and Antonio Castellanos. "Computer simulation of model cohesive powders: influence of assembling procedure and contact laws on low consolidation states". In: Physical review E 75.(1) (2007), p. 011303 (cit. on p. 21).

[69] Bruno Gilles and Christophe Coste. "Low-frequency behavior of beads constrained on a lattice". In: Physical review letters 90.(17) (2003), p. 174302 (cit. on p. 122). 
[70] JD GODDARD. "Nonlinear elasticity and pressure-dependent wave speeds in granular media". In: Proceedings of the Royal Society of London A: Mathematical, Physical and Engineering Sciences. Vol. 430. 1878. The Royal Society. 1990, pp. 105-131 (cit. on pp. 9, 49, 82).

[71] I Goldhirsch. "Rapid granular flows". In: Annu. Rev. Fluid Mech. 35 (2003), pp. 267-293 (cit. on p. 156).

[72] IsAAC GOLDHIRSCH. "Stress, stress asymmetry and couple stress: from discrete particles to continuous fields". In: Granular Matter 12.(3) (2010), pp. 239-252 (cit. on pp. 20, 54).

[73] FATiH GÖNCÜ, OREnCio DurÁn, and Stefan Luding. "Constitutive relations for the isotropic deformation of frictionless packings of polydisperse spheres". In: Comptes Rendus Mécanique 338.(10) (2010), pp. 570-586 (cit. on pp. 31, 32, 54).

[74] FATIH GÖNCÜ and STEFAn LUding. "Effect of particle friction and polydispersity on the macroscopic stress-strain relations of granular materials". In: Acta geotechnica 8.(6) (2013), pp. 629-643 (cit. on pp. 5, 32, 49, 58).

[75] Marcial Gonzalez and Alberto M Cuitiño. "A nonlocal contact formulation for confined granular systems". In: Journal of the Mechanics and Physics of Solids 60.(2) (2011), pp. 333-350 (cit. on p. 99).

[76] Marcial Gonzalez and Alberto M Cuitiño. "Microstructure evolution of compressible granular systems under large deformations". In: Journal of the Mechanics and Physics of Solids 93 (2016), pp. 44-56 (cit. on pp. 99, 107).

[77] PAUl D GReEning and David FT Nash. "Frequency domain determination of G 0 using bender elements". In: Geotechnical Testing Journal 27.(3) (2004), pp. 288-294 (cit. on p. 89).

[78] Ning Guo and Jidong ZHAo. "A coupled FEM/DEM approach for hierarchical multiscale modelling of granular media". In: International Journal for Numerical Methods in Engineering 99.(11) (2014), pp. 789-818. DOI: $10.1002 / \mathrm{nme} .4702$ (cit. on pp. 163, 164).

[79] MA GuTIÉRREZ and R DE BORST. "Computational models for failure in cohesive-frictional materials with stochastically distributed imperfections". In: Springer, 2001, pp. 1-16 (cit. on p. 20). 
[80] B.O. HARDIN and W.L. BLACK. "Elastic wave velocities in granular soils". In: Journal of Soil Mechanics and Foundations Division 92.(2) (1966), pp. 27-42 (cit. on p. 9).

[81] B.O. HARDIN and F.E.JR RICHART. "Elastic wave velocities in granular soils". In: Journal of Soil Mechanics and Foundations Division 89.(1) (1963), pp. 33-66 (cit. on p. 9).

[82] B Harthong, J-F JÉrier, P DorÉmus, D Imbault, and F-V Donzé. "Modeling of high-density compaction of granular materials by the discrete element method". In: International Journal of Solids and Structures 46.(18) (2009), pp. 3357-3364 (cit. on p. 98).

[83] Martin Haustein, Anton Gladkyy, and RÜdiger Schwarze. "Discrete element modeling of deformable particles in YADE". In: SoftwareX 6 (2017), pp. 118-123 (cit. on pp. 99, 107).

[84] Andong He and John S Wettlaufer. "Hertz beyond belief". In: Soft matter 10.(13) (2014), pp. 2264-2269 (cit. on p. 107).

[85] Thor C Heimdahl and ANDrew Drescher. "Elastic anisotropy of tire shreds". In: JGGE 125.(5) (1999), pp. 383-389 (cit. on pp. 3, 76).

[86] SinAN HinislioĞLU and EMINE AĞAR. "Use of waste high density polyethylene as bitumen modifier in asphalt concrete mix". In: Materials letters 58.(3-4) (2004), pp. 267-271 (cit. on p. 3).

[87] M HiraiWa, SP WAllen, and N Boechler. "Acoustic wave propagation in disordered microscale granular media under compression". In: Granular Matter 19.(3) (2017), p. 62 (cit. on p. 123).

[88] RYAN C HuRley and José E ANDRADE. "Friction in inertial granular flows: competition between dilation and grain-scale dissipation rates". In: Granular Matter 17.(3) (2015), pp. 287-295 (cit. on p. 35).

[89] Ilaria iaconeta, Antonia Larese, Riccardo Rossi, and Eugenio OÑATE. "An implicit material point method applied to granular flows". In: Procedia Engineering 175 (2017), pp. 226-232 (cit. on pp. 99, 165).

[90] OluKayode I IMOLE and ET AL. "Experiments and discrete element simulation of the dosing of cohesive powders in a simplified geometry". In: Powder technology 287 (2016), pp. 108-120 (cit. on p. 20).

[91] Olukayode I Imole, Nishant Kumar, Vanessa Magnanimo, and SteFAN LUDING. "Hydrostatic and shear behavior of frictionless granular assemblies under different deformation conditions". In: KONA Powder and Particle Journal 30 (2013), pp. 84-108 (cit. on pp. 51, 54, 56). 
[92] Olukayode I Imole, Mateusz Wojtkowski, Vanessa Magnanimo, and STEFAN LUDING. "Micro-macro correlations and anisotropy in granular assemblies under uniaxial loading and unloading". In: Physical Review E 89.(4) (2014), p. 042210 (cit. on pp. 29, 54).

[93] J Jenkins, D Johnson, L La Ragione, and H MaKse. "Fluctuations and the effective moduli of an isotropic, random aggregate of identical, frictionless spheres". In: Journal of the Mechanics and Physics of Solids 53.(1) (2005), pp. 197-225 (cit. on pp. 10, 49, 61, 70).

[94] XIAOPING JiA. "Codalike multiple scattering of elastic waves in dense granular media". In: Physical Review Letters 93.(15) (2004), p. 154303 (cit. on pp. 49, 60).

[95] Xiaoping Jia, C Caroli, and B Velicky. "Ultrasound propagation in externally stressed granular media". In: Physical Review Letters 82.(9) (1999), p. 1863 (cit. on p. 77).

[96] XiaOPing JiA and Pierre Mills. "Sound propagation in dense granular materials". In: Powders and Grains 2001. Balkema. 2001, pp. 105-112 (cit. on p. 76).

[97] D L Johnson, K Kendall, and A D RoberTs. "Surface energy and the contact of elastic solids". In: Proc. R. Soc. London A 324 (1971), pp. 301313 (cit. on p. 25).

[98] Kenneth Langstreth Johnson and Kenneth LangStRETH JOHNSON. Contact mechanics. Cambridge university press, 1987 (cit. on p. 101).

[99] PAUl A Johnson and Xiaoping JiA. "Nonlinear dynamics, granular media and dynamic earthquake triggering". In: Nature 437.(7060) (2005), p. 871 (cit. on pp. 14, 77).

[100] DAvid Hervey Johnston, MN Toksöz, and A Timur. "Attenuation of seismic waves in dry and saturated rocks: II. Mechanisms". In: Geophysics 44.(4) (1979), pp. 691-711 (cit. on p. 91).

[101] D Jongmans and M CAmpillo. "The determination of soil attenuation by geophysical prospecting and the validity of measured $\mathrm{Q}$ values for numerical simulations". In: Soil dynamics and earthquake engineering 12.(3) (1993), pp. 149-157 (cit. on pp. 77, 91, 142).

[102] Pierre Jop, YoËl Forterre, and Olivier Pouliquen. "A constitutive law for dense granular flows". In: Nature 441.(7094) (2006), pp. 727730 (cit. on pp. 35, 157). 
[103] N. G. VAN KAMPEN. Stochastic processes in physics and chemistry. Vol. 1. Elsevier, 1992 (cit. on p. 123).

[104] Gaetan Kerschen, Jean-claude Golinval, Alexander F Vakakis, and LAWRENCE A BERGMAN. "The method of proper orthogonal decomposition for dynamical characterization and order reduction of mechanical systems: an overview". In: Nonlinear dynamics 41.(1-3) (2005), pp. 147-169 (cit. on p. 131).

[105] Mohamed Hassan Khalili, Jean-Noël Roux, Jean-Michel Pereira, SÉBASTIEN BRISARD, and MiCHEL BORNERT. "Numerical study of onedimensional compression of granular materials. I. Stress-strain behavior, microstructure, and irreversibility". In: Physical Review E 95.(3) (2017), p. 032907 (cit. on pp. 49, 58).

[106] Robert Kievitsbosch, Hendrik Smit, Vanessa Magnanimo, Stefan LUDiNG, and KiANOOSH TAGHIZADEH. "Influence of dry cohesion on the micro-and macro-mechanical properties of dense polydisperse powders \& grains". In: EPJ Web of Conferences. Vol. 140. EDP Sciences. 2017, p. 08016 (cit. on pp. 21, 29).

[107] Eunho Kim, Alejandro J Martínez, Sean E Phenisee, PG KEVREKIDIS, MASON A PORTER, and JinKYU YANG. "Direct measurement of superdiffusive energy transport in disordered granular chains". In: Nature communications 9.(1) (2018), p. 640 (cit. on p. 91).

[108] H-K KIM and JC SANTAMARINA. "Sand-rubber mixtures (large rubber chips)". In: Canadian Geotechnical Journal 45.(10) (2008), pp. 14571466 (cit. on pp. 3, 76).

[109] Christoph Kloss, Christoph Goniva, Alice Hager, Stefan AmBERGER, and STEFAN PIRKER. "Models, algorithms and validation for opensource DEM and CFD-DEM". In: Progress in Computational Fluid Dynamics, an International Journal 12.(2-3) (2012), pp. 140-152 (cit. on p. 106).

[110] Christoph Kloss, Arno Mayrhofer, and Christoph Goniva. "Discrete element modelling of cohesive partiles under high pressure". 9th International Conference for Conveying and Handling of Particulate Solids, 2018, London, UK. unpublished thesis (cit. on p. 99).

[111] MA KoENDERs. "The incremental stiffness of an assembly of particles". In: Acta Mechanica 70.(1-4) (1987), pp. 31-49 (cit. on p. 61). 
[112] Lou Kondic. "Energy propagation through dense granular systems". In: Granular Matter 21.(4) (2019), p. 85 (cit. on p. 122).

[113] N. KruYT and L. Rothenburg. "Micromechanical bounds for the effective elastic moduli of granular materials". In: International Journal of Solids and Strucures 39.(2) (2002), pp. 311-324 (cit. on p. 160).

[114] N. P. KRUYT and L. RothenbuRg. "Micromechanical Definition of the Strain Tensor for Granular Materials". In: J. Appl. Mech. 63.(3) (1996), pp. 706-711. DOI: 10.1115/1.2823353 (cit. on p. 37).

[115] NicolaAs P KRUYT. "Micromechanical study of elastic moduli of threedimensional granular assemblies". In: International journal of solids and structures 51.(13) (2014), pp. 2336-2344 (cit. on p. 60).

[116] NP KRuYt, I Agnolin, S Luding, and L Rothenburg. "Micromechanical study of elastic moduli of loose granular materials". In: Journal of the Mechanics and Physics of Solids 58.(9) (2010), pp. 1286-1301 (cit. on pp. $49,61,62,72$ ).

[117] N.P. KruYt and L. Rothemburg. "Micromechanical Definition of the Strain Tensor for Granular Materials". In: Journal of Applied Mechanics 63.(3) (1996), pp. 706-711 (cit. on p. 160).

[118] NP KruYT and L Rothenburg. "Microinechanical Definition of the Strain Tensor for Granular Materials". In: Appi. Mech 118 (1996), pp. 706-711 (cit. on p. 60).

[119] N.P. KRUYT and L. RothenBURG. "Statistics of the elastic behaviour of granular materials". In: International Journal of Solids and Strucures 38 (2001), pp. 4879-4899 (cit. on pp. 160, 161).

[120] Matthew R Kuhn and Katalin Bagi. "Specimen size effect in discrete element simulations of granular assemblies". In: Journal of Engineering Mechanics 135.(6) (2009), pp. 485-492 (cit. on p. 72).

[121] N. Kumar, S. Luding, and V. Magnanimo. "Macroscopic model with anisotropy based on micro-macro information". In: Acta mechanica 225.(8) (2014), pp. 2319-2343 (cit. on pp. 7, 21, 56, 86).

[122] Nishant KumAR and Stefan Luding. "Memory of jamming-multiscale models for soft and granular matter". In: Granular Matter 18.(3) (2016), p. 58 (cit. on pp. 37, 48, 54). 
[123] Nishant Kumar, Vanessa Magnanimo, Marco Ramaioli, and SteFAN LUDING. "Tuning the bulk properties of bidisperse granular mixtures by small amount of fines". In: Powder technology 293 (2016), pp. 94112 (cit. on p. 3).

[124] R. KUWANO and JARDINE R.J. "On the applicability of cross-anisotropic elasticity to granular materials at very small strains". In: Géotechnique 52.(10) (2002), pp. 727-749 (cit. on p. 9).

[125] Luigi LA RAgione and James T Jenkins. "The initial response of an idealized granular material”. In: 463.(2079) (2007), pp. 735-758 (cit. on pp. $10,49,60,61,70)$.

[126] Luigi La Ragione and Vanessa Magnanimo. "Contact anisotropy and coordination number for a granular assembly: A comparison of distinctelement-method simulations and theory". In: Physical Review E 85.(3) (2012), p. 031304 (cit. on p. 56).

[127] C LABRA, JY OOI, and J SUN. "Spatial and temporal coarse-graining for DEM analysis". In: AIP Conference Proceedings. Vol. 1542. 1. AIP. 2013, pp. 1258-1261 (cit. on p. 105).

[128] P. V. LADE. "Copyright ASCE 20051 Soil Constitutive Models Evaluation, Selection, and Calibration". In: (2005), pp. 1-34 (cit. on p. 158).

[129] W. Michael Lai, David Rubin, and ERhard Krempl. "Chapter 1 - Introduction". In: Introduction to Continuum Mechanics. 2010, pp. 1-2. DOI: $10.1016 /$ B978-0-7506-8560-3.00001-3 (cit. on p. 157).

[130] L. D. LANDAU and EvgeniǏ MikhaǏLOVICH Lifshitz. Theory of elasticity. Pergamon Press, 1970 (cit. on pp. 124, 125, 149).

[131] B. P. LAWNEY and S. LUDING. "Frequency filtering in disordered granular chains". English. In: Acta Mechanica 225.(8) (2014), pp. 2385-2407. DOI: 10.1007 / s00707-014-1130-4 (cit. on pp. 122, 128, 144, 146, 149).

[132] BRIAN P LAWNEY and StEFAN LUding. "Mass-disorder effects on the frequency filtering in one-dimensional discrete particle systems". In: AIP Conference Proceedings. Vol. 1542. 1. AIP. 2013, pp. 535-538 (cit. on p. 122).

[133] Changho Lee, Hosung Shin, and Jong-Sub Lee. "Behavior of sandrubber particle mixtures: experimental observations and numerical simulations". In: International Journal for Numerical and Analytical Methods in Geomechanics 38.(16) (2014), pp. 1651-1663 (cit. on p. 76). 
[134] JH Lee, R Salgado, A Bernal, and CW Lovell. "Shredded tires and rubber-sand as lightweight backfill”. In: JGGE 125.(2) (1999), pp. 132141 (cit. on pp. 3, 76).

[135] Jong-Sub Lee, Jake Dodds, and J Carlos Santamarina. "Behavior of rigid-soft particle mixtures". In: Journal of materials in civil engineering 19.(2) (2007), pp. 179-184 (cit. on pp. 3, 76).

[136] Jong-Sub LeE and J Carlos Santamarina. "Bender elements: performance and signal interpretation". In: Journal of geotechnical and geoenvironmental engineering 131.(9) (2005), pp. 1063-1070 (cit. on p. 94).

[137] YC LiAnG, HP LeE, SP LiM, WZ Lin, KH LeE, and CG WU. "Proper orthogonal decomposition and its applications-Part I: Theory". In: Journal of Sound and vibration 252.(3) (2002), pp. 527-544 (cit. on p. 131).

[138] YC Liang, WZ Lin, HP LeE, SP Lim, KH LeE, and H Sun. "Proper orthogonal decomposition and its applications-part II: Model reduction for MEMS dynamical analysis". In: Journal of Sound and Vibration 256.(3) (2002), pp. 515-532 (cit. on p. 131).

[139] A.E.H. LovE. A Treatise on the Mathematical Theory of Elasticity. Cambridge University Press, Cambridge, 1927 (cit. on p. 9).

[140] Z. Lu, J.C. JofRIET, and S.C. NeGi. "A Numerical Model for Flow of Granular Materials in Silos. Part 1: Parametric Study". In: Journal of Agricultural Engineering Research 68 (1997), pp. 223-229. DOI: 10 . 1006/jaer. 1997.0198 (cit. on pp. 163, 164).

[141] S. LUDING. "About contact force-laws for cohesive frictional materials in $2 \mathrm{~d}$ and 3d". In: Behavior of granular media 9 (2006), pp. 137-147 (cit. on p. 20).

[142] S. Luding. "Cohesive, frictional powders: contact models for tension". In: Granular matter 10.(4) (2008), pp. 235-246 (cit. on pp. 20, 21, 24, $85,86)$.

[143] S. LUDING. "Collisions \& contacts between two particles". In: Physics of dry granular media. Springer, 1998, pp. 285-304 (cit. on p. 20).

[144] S. Luding. "The Effect of Friction on Wide Shear Bands". In: Particulate Science and Technology 26.(1) (2007), pp. 33-42. DOI: 10.1080 / 02726350701759167 (cit. on p. 157).

[145] S Luding. "Towards dense, realistic granular media in 2D". In: Nonlinearity 22.(12) (2009), R101-R146. DOI: 10.1088/0951-7715/22/12/ R01 (cit. on p. 6). 
[146] S Luding and E S Perdahciouglu. "A Local Constitutive Model with Anisotropy for Various Homogeneous 2D Biaxial Deformation Modes". In: Chemie Ingenieur Technik 83.(5) (2011), pp. 672-688 (cit. on p. 7).

[147] STEFAn Luding. "From molecular dynamics and particle simulations towards constitutive relations for continuum theory". In: Springer, 2009, pp. 453-492 (cit. on p. 20).

[148] STEFAn LUding. "Granular matter: So much for the jamming point". In: Nature physics 12.(6) (2016), p. 531 (cit. on pp. 3, 48, 54, 98).

[149] STEFAN LUDING. "Introduction to discrete element methods : basic of contact force models and how to perform the micro-macro transition to continuum theory". In: European Journal of Environmental and Civil Engineering 12.(7-8) (2008), pp. 785-826 (cit. on pp. 20, 22, 24, 123).

[150] STEFAN LUDING. "Introduction to discrete element methods: basic of contact force models and how to perform the micro-macro transition to continuum theory". In: European Journal of Environmental and Civil Engineering 12.(7-8) (2008), pp. 785-826 (cit. on pp. 20, 50, 51, 85, 98, 101).

[151] Stefan Luding. "Introduction to Discrete Element Methods. Basics of Contact Force Models and how to perform the Micro-Macro Transition to Continuum Theory". In: European Journal of Environmental and Civil Engineering 12.(7-8) (2008), pp. 785-826 (cit. on pp. 98, 105).

[152] Stefan Luding and Fernando Alonso-Marroquín. "The criticalstate yield stress (termination locus) of adhesive powders from a single numerical experiment". In: Granul. Matter. 13 (2011), pp. 109-119. DOI: $10.1007 / \mathrm{s} 10035-010-0241-4$ (cit. on p. 157).

[153] Stefan Luding and J Tomas. "Particles, contacts, bulk-behavior". In: Granular matter 16.(3) (2014), pp. 279-280 (cit. on p. 2).

[154] V Magnanimo. "Micromechanics of complex granular materials: a focus on small strain behavior". In: EPJ Web of Conferences. Vol. 140. EDP Sciences. 2017, p. 01010 (cit. on p. 4).

[155] V Magnanimo, L La Ragione, JT Jenkins, P Wang, and HA Makse. "Characterizing the shear and bulk moduli of an idealized granular material". In: EPL (Europhysics Letters) 81.(3) (2008), p. 34006 (cit. on pp. $9,49,56,60,62,82,86)$. 
[156] V. Magnanimo, L. La Ragione, J. T. Jenkins, P. Wang, and H. A. MAKSE. "Characterizing the shear and bulk moduli of an idealized granular material". In: EPL (Europhysics Letters) 81.(3) (2008), p. 34006 (cit. on p. 42).

[157] RJ MAIR. "Developments in geotechnical engineering research: application to tunnels and deep excavations". In: Proceedings of Institution of Civil Engineers: Civil Engineering. Vol. 93. 1993, pp. 27-41 (cit. on pp. 7, 8).

[158] H.A. Makse, N. Gland, D.L. Johnson, and L. Schwartz. "Why effective medium theory fails in granular materials". In: Physical Review Letters 83 (1999), p. 5070 (cit. on p. 9).

[159] Hernán A Makse, Nicolas Gland, David L Johnson, and LaWrence SCHWARTZ. "Granular packings: Nonlinear elasticity, sound propagation, and collective relaxation dynamics". In: Physical Review E 70.(6) (2004), p. 061302 (cit. on pp. 49, 60, 70, 76, 82).

[160] Hernán A MaKse, Nicolas Gland, David L Johnson, and LaWrence M SCHWARTZ. "Why effective medium theory fails in granular materials". In: Physical Review Letters 83.(24) (1999), p. 5070 (cit. on pp. 9, 49, 62, 70).

[161] G MARKetos and C O'Sullivan. "A micromechanics-based analytical method for wave propagation through a granular material". In: Soil Dynamics and Earthquake Engineering 45 (2013), pp. 25-34 (cit. on p. 123).

[162] C. L. MARTin, D. Bouvard, and S. Shima. "Study of particle rearrangement during powder compaction by the Discrete Element Method". In: Journal of the Mechanics and Physics of Solids 51.(4) (2003), pp. 667693 (cit. on p. 98).

[163] Marwa Masmoudi, StÉphane Job, Mohamed Slim Abbes, Imad TaWFIQ, and MOHAMED HADDAR. "Experimental and numerical investigations of dissipation mechanisms in particle dampers". In: Granular Matter 18.(3) (2016), p. 71 (cit. on p. 142).

[164] Arno Mayrhofer, Alice Hager, and Christoph Kloss. "Energy balance in a particle system". In: Sept. 2017 (cit. on p. 108).

[165] A MERKEL and Stefan LUding. "Enhanced micropolar model for wave propagation in ordered granular materials". In: International Journal of Solids and structures 106 (2017), pp. 91-105 (cit. on pp. 49, 60). 
[166] A Merkel, V Tournat, and V Gusev. "Dispersion of elastic waves in three-dimensional noncohesive granular phononic crystals: properties of rotational modes". In: Physical Review E 82.(3) (2010), p. 031305 (cit. on p. 122).

[167] G D R MiDi. "On dense granular flows". In: Eur. Phys. J. E 14.(4) (2004), pp. 341-365. DOI: 10.1140 / epje/i2003-10153-0 (cit. on p. 157).

[168] ANIL MisRa and Ching S CHANG. "Effective elastic moduli of heterogeneous granular solids". In: International Journal of Solids and Structures 30.(18) (1993), pp. 2547-2566 (cit. on pp. 49, 60).

[169] N. Mitarai and F. Nori. "Wet granular materials". In: Advances in Physics 55.(1-2) (2006), pp. 1-45 (cit. on p. 21).

[170] Guilhem MolLon. "Mixtures of hard and soft grains: micromechanical behavior at large strains". In: Granular Matter 20.(3) (2018), p. 39 (cit. on p. 76).

[171] O Mouraille and S Luding. "Mechanic waves in sand: effect of polydispersity". In: Partec. Vol. 2007. 2007 (cit. on p. 122).

[172] O Mouraille and StEFAn Luding. "Sound wave propagation in weakly polydisperse granular materials". In: Ultrasonics 48.(6-7) (2008), pp. 498-505 (cit. on pp. 42, 49, 60, 94, 122).

[173] O Mouraille, WA Mulder, and S Luding. "Sound wave acceleration in granular materials". In: Journal of Statistical Mechanics: Theory and Experiment 2006.(07) (2006), P07023 (cit. on pp. 42, 94).

[174] ORION MOURAILlE. "Sound propagation in dry granular materials : discrete element simulations, theory, and experiments". PhD thesis. EMPTY, Enschede, 2009 (cit. on pp. 146, 148).

[175] D. Muir Wood. "Life cycles of granular materials". In: Philosophical Transactions of Royal Society London A 356.(1747) (1998), pp. 24532470 (cit. on p. 7).

[176] R M Nedderman. Statics and kinematics of granular materials. Cambr. Univ. Press, Cambridge, 1992 (cit. on p. 156).

[177] S.C. Negi, Z. LU, and J.C. JofRIET. "A Numerical Model for Flow of Granular Materials in Silos. Part 2: Parametric Study". In: Journal of Agricultural Engineering Research 68 (1997), pp. 231-236. DOI: 10 . 1006/jaer.1997.0198 (cit. on p. 164). 
[178] Saeid Nezamabadi, Farhang Radjai, Julien Averseng, and JeanYVES DELENNE. "Modeling soft granular media". In: Modeling Granular Media Across Scales-MGMAS2014. 2014 (cit. on p. 99).

[179] T K Nguyen, G Combe, D CAillerie, and J Desrues. "Modeling of a cohesive granular materials by a multi-scale approach Modeling of a Cohesive Granular Materials by a Multi-scale Approach”. In: 1194 (2013), pp. 9-12. DOI: 10.1063/1.4812151 (cit. on p. 162).

[180] AN NORRIS and DL JOHNSON. "Nonlinear elasticity of granular media". In: Journal of Applied Mechanics 64.(1) (1997), pp. 39-49 (cit. on p. 49).

[181] SRdjan Ostojic, Ellák Somfai, and Bernard Nienhuis. "Scale invariance and universality of force networks in static granular matter". In: Nature 439.(7078) (2006), p. 828 (cit. on p. 122).

[182] Michio Otsuki and Hisao HaYAKAWA. "Discontinuous change of shear modulus for frictional jammed granular materials". In: Physical Review E 95.(6) (2017), p. 062902 (cit. on p. 58).

[183] J O'Donovan, E Ibraim, C O'Sullivan, S Hamlin, D Muir Wood, and G MARKETOS. "Micromechanics of seismic wave propagation in granular materials". In: Granular Matter 18.(3) (2016), p. 56 (cit. on p. 94).

[184] J O'Donovan, C O'Sullivan, G Marketos, and D Muir Wood. "Analysis of bender element test interpretation using the discrete element method". In: Granular Matter 17.(2) (2015), pp. 197-216 (cit. on pp. 94, 95).

[185] John H Page, IP Jones, Henry P Schriemer, Michael L Cowan, Ping Sheng, and DAvid A Weitz. "Diffusive transport of acoustic waves in strongly scattering media". In: Physica B: Condensed Matter 263 (1999), pp. 37-39 (cit. on pp. 91, 142).

[186] Raj Kumar Pal, Amnaya P Awasthi, and Philippe H Geubelle. "Wave propagation in elasto-plastic granular systems". In: Granular Matter 15.(6) (2013), pp. 747-758 (cit. on p. 76).

[187] Raj Kumar Pal, Jeremy Morton, Erheng Wang, John Lambros, and Philippe H Geubelle. "Impact response of elasto-plastic granular chains containing an intruder particle". In: Journal of Applied Mechanics 82.(1) (2015), p. 011002 (cit. on p. 77). 
[188] Sibel Pamukcu and Suat Akbulut. "Thermoelastic enhancement of damping of sand using synthetic ground rubber". In: Journal of Geotechnical and Geoenvironmental Engineering 132.(4) (2006), pp. 501-510 (cit. on pp. 76, 77, 142).

[189] M Pasha, S Dogbe, C Hare, A Hassanpour, and M Ghadiri. "A linear model of elasto-plastic and adhesive contact deformation". In: Granul. Matter. 16.(1) (2014), pp. 151-162. DOI: 10.1007/s10035-013-0476y (cit. on p. 25).

[190] Ann-Sofie Persson and Göran Frenning. "An experimental evaluation of the accuracy to simulate granule bed compression using the discrete element method". In: Powder technology 219 (2012), pp. 249256 (cit. on p. 98).

[191] P. Philippe and D. Bideau. "Compaction Dynamics Of A Granular Medium Under Vertical Tapping”. In: Europhysics Letters 60.(5) (2002), p. 677 (cit. on p. 9).

[192] LaWrence T Pillage and Ronald A Rohrer. "Asymptotic waveform evaluation for timing analysis". In: IEEE Transactions on Computer-Aided Design of Integrated Circuits and Systems 9.(4) (1990), pp. 352-366 (cit. on p. 131).

[193] Steve Plimpton. "Fast parallel algorithms for short-range molecular dynamics". In: Journal of computational physics 117.(1) (1995), pp. 119 (cit. on p. 106).

[194] D PORTNIKOV and H KALMAN. "Determination of elastic properties of particles using single particle compression test". In: Powder technology 268 (2014), pp. 244-252 (cit. on p. 77).

[195] Randi Potekin, D Michael McFarland, and Alexander F Vakakis. "Nonlinear wave scattering at the flexible interface of a granular dimer chain". In: Granular Matter 18.(3) (2016), p. 68 (cit. on p. 123).

[196] AdAm T Procopio and Antonios Zavaliangos. "Simulation of multiaxial compaction of granular media from loose to high relative densities". In: Journal of the Mechanics and Physics of Solids 53.(7) (2005), pp. 1523-1551 (cit. on p. 98). 
[197] AO RAJI and JF FAVIER. "Model for the deformation in agricultural and food particulate materials under bulk compressive loading using discrete element method. I: Theory, model development and validation". In: Journal of food engineering 64.(3) (2004), pp. 359-371 (cit. on p. 98).

[198] DenNis C RAPAPORT. The art of molecular dynamics simulation. Cambridge university press, 2004, p. 11 (cit. on p. 20).

[199] John William Strutt Baron Rayleigh. The theory of sound. Vol. 2. Macmillan, 1896 (cit. on p. 131).

[200] AleXander Ries, Lothar Brendel, and Dietrich E Wolf. "Shearrate diffusion and constitutive relations during transients in simple shear". In: Comp. Part. Mech. (2015), pp. 1-8 (cit. on p. 157).

[201] S.K. RoESLER. "Anisotropic shear modulus due to stress anisotropy". In: Journal of Geotechnical Engineering 105.(7) (1979), pp. 871-880 (cit. on p. 9).

[202] Jerzy RoJek, Aleksander Zubelewicz, Nikhil Madan, and Szymon NosEwicz. "The discrete element method with deformable particles". In: International Journal for Numerical Methods in Engineering 114.(8) (2018), pp. 828-860 (cit. on p. 99).

[203] L. Rothenburg and A.P.S. Selvadurai. "A micromechanical definition of the Cauchy stress for particulate media". In: Proceedings International Symposium on Mechanical Behaviour of Structured Media, Ottawa, Canada. 1981, pp. 469-486 (cit. on p. 159).

[204] Sudeshna Roy, Stefan Luding, and Thomas Weinhart. "Towards hydrodynamic simulations of wet particle systems". In: Procedia Engineering 102 (2015), pp. 1531-1538 (cit. on p. 157).

[205] Sudeshna Roy, AbHinendra Singh, Stefan Luding, and Thomas WEINHART. "Micro-macro transition and simplified contact models for wet granular materials". In: CPM (2015), pp. 1-14 (cit. on p. 21).

[206] Wolfgang Sachse and Yih-Hsing Pao. "On the determination of phase and group velocities of dispersive waves in solids". In: Journal of applied Physics 49.(8) (1978), pp. 4320-4327 (cit. on p. 142). 
[207] Martin H. Sadd, Qiming TAi, and Arun Shukla. "Contact law effects on wave propagation in particulate materials using distinct element modeling". In: International Journal of Non-Linear Mechanics 28.(2) (1993), pp. 251 -265. DOI: http ://dx . doi .org/10 . 1016/0020 7462 (93) 90061-0 (cit. on p. 22).

[208] JC SANTAMARINA and M Aloufi. "Small strain stiffness: A micromechanical experimental study". In: Proceedings of Pre-failure Deformation Characteristics of Geomaterials (1999), pp. 451-458 (cit. on p. 76).

[209] Haruo Sato, Michael C Fehler, and TAKuto Maeda. Seismic wave propagation and scattering in the heterogeneous earth. Vol. 496. Springer, 2012 (cit. on p. 122).

[210] AuckPath SAWAngsuriYa. "Wave Propagation Methods for Determining Stiffness of Geomaterials". In: Wave Processes in Classical and New Solids. IntechOpen, 2012 (cit. on p. 76).

[211] John A ScAles and ERIK S VAN Vleck. "Lyapunov exponents and localization in randomly layered media". In: Journal of Computational physics 133.(1) (1997), pp. 27-42 (cit. on p. 122).

[212] Carl F Schreck, Corey S O'Hern, and Mark D Shattuck. "Vibrations of jammed disk packings with Hertzian interactions". In: Granular Matter 16.(2) (2014), pp. 209-216 (cit. on p. 124).

[213] FrEDERICK M SEARS and BRIAN P BonNER. "Ultrasonic attenuation measurement by spectral ratios utilizing signal processing techniques". In: IEEE Transactions on geoscience and remote sensing (2) (1981), pp. 95-99 (cit. on pp. 91, 123).

[214] RAJEEV SENAPATI and JiAnMEI ZHANG. "Identifying fracture origin in ceramics by combination of nondestructive testing and discrete element analysis". In: AIP Conference Proceedings. Vol. 1211. 1. AIP. 2010, pp. 1445-1451 (cit. on p. 98).

[215] M Reza Shaebani, Mahyar Madadi, Stefan Luding, and Dietrich E WOLF. "Influence of polydispersity on micromechanics of granular materials”. In: Physical Review E 85.(1) (2012), p. 011301 (cit. on p. 60).

[216] PING SHENG. Introduction to wave scattering, localization and mesoscopic phenomena. Vol. 88. Springer Science \& Business Media, 2006 (cit. on p. 122). 
[217] T SHIRE, C O'sullivan, and KJ HANLEY. "The influence of fines content and size-ratio on the micro-scale properties of dense bimodal materials". In: Granular Matter 18.(3) (2016), p. 52 (cit. on p. 3).

[218] R. K. ShrivastaVA and S. Luding. "Effect of disorder on bulk sound wave speed: a multiscale spectral analysis". In: Nonlinear Processes in Geophysics 24.(3) (2017), pp. 435-454. DOI: 10 . 5194/npg-24-4352017 (cit. on pp. 128, 136).

[219] R. K. SHRivastaVA and S. LUDing. "Wave propagation of spectral energy content in a granular chain". In: EPJ Web of Conferences. Vol. 140. EDP Sciences. 2017, p. 02023. DOI: 10.1051/epjconf/201714002023 (cit. on p. 128).

[220] Rohit Kumar Shrivastava and Stefan Luding. "Effect of disorder on bulk sound wave speed: a multiscale spectral analysis". In: Nonlinear processes in geophysics 24.(3) (2017), p. 435 (cit. on pp. 144, 146).

[221] Luc Sibille, François Nicot, Frédéric-Victor Donzé, and FÉliX DARVE. "Analysis of failure occurrence from direct simulations". In: $\mathrm{Eu}$ ropean Journal of Environmental and Civil Engineering 13.(2) (2009), pp. 187-201 (cit. on p. 49).

[222] RAFAT SiddiQue and TARUn R NaIK. "Properties of concrete containing scrap-tire rubber-an overview". In: Waste management 24.(6) (2004), pp. 563-569 (cit. on p. 3).

[223] A Singh, V Magnanimo, and S Luding. In: AIP Conference Proceedings 1542.(1) (2013), pp. 682-685 (cit. on pp. 21, 24).

[224] A. Singh, V. MAGNANimo, and S. Luding. "A contact model for sticking of adhesive mesoscopic particles". In: (2015) (cit. on pp. 24, 25).

[225] Abhinendra Singh, Vanessa Magnanimo, Kuniyasu Saitoh, and STEFAN LUDING. "Effect of cohesion on shear banding in quasistatic granular materials". In: Physical Review E 90.(2) (2014), p. 022202 (cit. on p. 20).

[226] Abhinendra Singh, Vanessa Magnanimo, Kuniyasu Saitoh, and STEFAN LUDING. "The role of gravity or pressure and contact stiffness in granular rheology". In: New journal of physics 17.(4) (2015), p. 043028 (cit. on p. 20). 
[227] Abhinendra Singh, Vanessa Magnanimo, Kuniyasu Saitoh, and STEFAN LUDING. "The role of gravity or pressure and contact stiffness in granular rheology". In: New J. Phys. 17.(4) (2015), p. 043028 (cit. on p. 157).

[228] E. Somfai, J.N. Roux, J.H. Snoeijer, M. van Hecke, and W. van SAARLOOS. "Elastic Wave Propagation in Confined Granular Systems". In: Physical Review E 72.(2) (2005), p. 021301 (cit. on p. 12).

[229] Ellák Somfai, JeAn-NoËl RouX, Jacco H SnOeiJer, Martin VAN HECKE, and WIM VAN SAARLOOS. "Elastic wave propagation in confined granular systems". In: Physical Review E 72.(2) (2005), p. 021301 (cit. on pp. 49, 82).

[230] CM Sorace, MY Louge, MD Crozier, and VHC LaW. "High apparent adhesion energy in the breakdown of normal restitution for binary impacts of small spheres at low speed". In: Mechanics Research Communications 36.(3) (2009), pp. 364-368 (cit. on p. 25).

[231] JAn Stransky. "Mesoscale Discrete Element Model for Concrete and Its Combination with FEM". PhD thesis. Czech Technical University in Prague, 2018 (cit. on p. 99).

[232] K. TAghizadeH. "Micromechanical study of the elastic stiffness in frictional granular materials". English. In: (2015) (cit. on p. 21).

[233] K Taghizadeh, N Kumar, V Magnanimo, and S Luding. "Understanding the effects of inter-particle contact friction on the elastic moduli of granular materials". In: IOP Conference Series: Earth and Environmental Science. Vol. 26. 1. IOP Publishing. 2015, p. 012008 (cit. on pp. 49, 54, $85,86)$.

[234] K TAghizadeh, S Luding, and V Magnanimo. "DEM applied to soil mechanics". In: ALERT Doctoral School 2017 Discrete Element Modeling (2017), p. 129 (cit. on pp. 20, 21, 29, 42, 101).

[235] Kianoosh Taghizadeh, Gä̈l Combe, and Stefan Luding. "ALERT Doctoral School 2017 Discrete Element Modeling". In: (2017) (cit. on p. 98).

[236] Kianoosh Taghizadeh, Holger Steeb, Vanessa Magnanimo, and STEFAN LUDING. "Elastic waves in particulate glass-rubber mixture: experimental and numerical investigations/studies". In: EPJ Web of Conferences. Vol. 140. EDP Sciences. 2017, p. 12019 (cit. on pp. 49, 76, 98). 
[237] YoICHI TATARA. "Extensive theory of force-approach relations of elastic spheres in compression and in impact". In: Journal of engineering materials and technology 111.(2) (1989), pp. 163-168 (cit. on pp. 110, 113).

[238] Colin Thornton, Sharen J Cummins, and Paul W Cleary. "An investigation of the comparative behaviour of alternative contact force models during elastic collisions". In: Powder Technology 210.(3) (2011), pp. 189-197 (cit. on p. 101).

[239] Colin Thornton, Sharen J Cummins, and Paul W Cleary. "An investigation of the comparative behaviour of alternative contact force models during inelastic collisions". In: Powder technology 233 (2013), pp. 30-46 (cit. on p. 102).

[240] Colin Thornton and Zemin Ning. "A theoretical model for the stick/bounce behaviour of adhesive, elastic-plastic spheres". In: Powder technology 99.(2) (1998), pp. 154-162 (cit. on p. 25).

[241] MN Toksöz, DAvid H Johnston, and AyTeKin Timur. "Attenuation of seismic waves in dry and saturated rocks: I. Laboratory measurements". In: Geophysics 44.(4) (1979), pp. 681-690 (cit. on p. 91).

[242] Jürgen TOMAs. "Fundamentals of cohesive powder consolidation and flow". In: Granular Matter 6.(2) (2004), pp. 75-86. DOI: 10.1007 / s10035-004-0167-9 (cit. on pp. 24, 25).

[243] Jürgen Tomas. "Particle Adhesion Fundamentals and Bulk Powder Consolidation". In: KONA 18 (2000), pp. 157-169. DOI: $10.14356 /$ kona. 2000022 (cit. on pp. 24, 25).

[244] RAINER TONN. "Comparison of seven methods for the computation of Q". In: Physics of the Earth and Planetary interiors 55.(3-4) (1989), pp. 259-268 (cit. on pp. 91, 142).

[245] RAINER TONN. "The determination of the seismic quality factor Q from VSP data: A comparison of different computational methods". In: Geophysical Prospecting 39.(1) (1991), pp. 1-27 (cit. on p. 91).

[246] V. Tournat, V. E. Gusev, and B. Castagnède. "Self-demodulation of elastic waves in a one-dimensional granular chain". In: Phys. Rev. E 70 (Iss. 5 2004), p. 056603. DOI: 10.1103/PhysRevE. 70.056603 (cit. on p. 135). 
[247] V TOuRnat and VE GusEv. "Acoustics of unconsolidated "model" granular media: An overview of recent results and several open problems". In: Acta Acustica united with Acustica 96.(2) (2010), pp. 208-224 (cit. on p. 122).

[248] Francesco TREntadue. "A micromechanical model for a non-linear elastic granular material based on local equilibrium conditions". In: International journal of solids and structures 38.(40-41) (2001), pp. 73197342 (cit. on p. 49).

[249] X. TU and J.E. ANDRADE. "Criteria for static equilibrium in particulate mechanics computations". In: International Journal for Numerical Methods in Engineering 75 (2008), pp. 1581-1606 (cit. on p. 158).

[250] R. TYкноNIUK and ET AL. "Ultrafine cohesive powders: From interparticle contacts to continuum behaviour". English. In: Chemical Engineering Science 62.(11) (2007), pp. 2843-2864 (cit. on p. 21).

[251] Julio R VAldes and T MATthew Evans. "Sand-rubber mixtures: experiments and numerical simulations". In: Canadian Geotechnical Journal 45.(4) (2008), pp. 588-595 (cit. on pp. 3, 76).

[252] Mirko VAN DER BAAN. "Acoustic wave propagation in one dimensional random media: the wave localization approach". In: Geophysical Journal International 145.(3) (2001), pp. 631-646 (cit. on p. 122).

[253] K WALton. "The effective elastic moduli of a random packing of spheres". In: Journal of the Mechanics and Physics of Solids 35.(2) (1987), pp. 213-226 (cit. on pp. 9, 49, 58).

[254] O. R. WALtON and R. L. BRAUn. "Viscosity, granular-temperature, and stress calculations for shearing assemblies of inelastic, frictional disks". In: Journal of Rheology 30 (1986), pp. 949-980. DOI: $10.1122 / 1$. 549893 (cit. on pp. 24, 25).

[255] O. R. Walton, R. L. Braun, T. R. Butkovich, and F.E. HeuzÉ. "Redirection of dynamic compressive Waves in Materials with nearly orthogonal and random joint sets". In: AMD 117 (1991), pp. 251 -265 (cit. on p. 22).

[256] Zong-Jun Wang, Si-Yuan CAO, HaO-RAN Zhang, Ying-Ming QU, DiAn YuAn, Jin-HAO YANG, and GUAN-Ming SHAO. "Estimation of quality factors by energy ratio method". In: Applied Geophysics 12.(1) (2015), pp. 86-92 (cit. on p. 91). 
[257] R. L. WEAVER. "Information from Seismic Noise". In: Science 307.(5715) (2005), pp. 1568-1569. DOI: 10.1126/science. 1109834 (cit. on p. 122).

[258] T Weinhart, R Hartkamp, A R Thornton, and S Luding. "Coarsegrained local and objective continuum description of three-dimensional granular flows down an inclined surface". In: Physics of Fluids 25.(7) (2013), p. 070605. DOI: 10.1063/1.4812809 (cit. on p. 157).

[259] Thomas Weinhart, Remco Hartkamp, Anthony R Thornton, and STEFAN LUDING. "Coarse-grained local and objective continuum description of three-dimensional granular flows down an inclined surface". In: Physics of Fluids 25.(7) (2013), p. 070605 (cit. on p. 105).

[260] S VAN DEN Wildenberg, M VAN Hecke, and X JiA. "Evolution of granular packings by nonlinear acoustic waves". In: EPL (Europhysics Letters) 101.(1) (2013), p. 14004 (cit. on p. 122).

[261] Siet Van den Wildenberg, Arnaud Tourin, and Xiaoping Jia. "Sound velocity fluctuations in confined granular materials: Coarsegraining lengths and elastic heterogeneities". In: EPL (Europhysics Letters) 115.(3) (2016), p. 34005 (cit. on p. 76).

[262] P. Wriggers. Computational Contact Mechanics. Springer, 2006, p. 521 (cit. on p. 165).

[263] Chuan-Yu Wu and Thorsten Pöschel. "Micro-mechanics and dynamics of cohesive particle systems". In: Granul. Matter. 15.(4) (2013), pp. 389-390 (cit. on p. 2).

[264] Feipeng Xiao, Serji Amirkhanian, and C Hsein JuAng. "Rutting resistance of rubberized asphalt concrete pavements containing reclaimed asphalt pavement mixtures". In: Journal of Materials in Civil Engineering 19.(6) (2007), pp. 475-483 (cit. on p. 3).

[265] Beichuan YAN, Richard A. REgueiro, and Stein Sture. "Threedimensional ellipsoidal discrete element modeling of granular materials and its coupling with finite element facets". In: Engineering Computations 27.(4) (2010), pp. 519-550. DOI: 10.1108/02644401011044603 (cit. on pp. 161, 163).

[266] RY YANG, RP Zou, AB YU, and SING-Ki CHoI. "Characterization of interparticle forces in the packing of cohesive fine particles". In: Physical Review E 78.(3) (2008), p. 031302 (cit. on p. 21). 
[267] Shiping Yang, Robert A Lohnes, and Bruce H KJartanson. "Mechanical properties of shredded tires". In: Geotechnical Testing Journal 25.(1) (2002), pp. 44-52 (cit. on p. 76).

[268] ZhanPING You and WG ButTlaR. "Discrete element modeling to predict the modulus of asphalt concrete mixtures". In: Journal of Materials in Civil Engineering 16.(2) (2004), pp. 140-146 (cit. on p. 3).

[269] YanXin Yu, Yi Pik Cheng, Xiaomin Xu, and Kenichi Soga. "Discrete element modelling of methane hydrate soil sediments using elongated soil particles". In: Computers and Geotechnics 80 (2016), pp. 397-409 (cit. on p. 3).

[270] HP ZHANG and HA MAKSE. "Jamming transition in emulsions and granular materials". In: Physical Review E 72.(1) (2005), p. 011301 (cit. on p. 101).

[271] Jie Zhang, Robert P Behringer, and IsaAc Goldhirsch. "Coarsegraining of a physical granular system". In: Progress of Theoretical Physics Supplement 184 (2010), pp. 16-30 (cit. on p. 105).

[272] Yijing Zhang, D Michael McFarland, and AleXander F VaKaKis. "Propagating discrete breathers in forced one-dimensional granular networks: theory and experiment". In: Granular Matter 19.(3) (2017), p. 59 (cit. on p. 123).

[273] Wei Zhou, Kun Xu, Gang Ma, Lifu Yang, and Xiaolin Chang. "Effects of particle size ratio on the macro-and microscopic behaviors of binary mixtures at the maximum packing efficiency state". In: Granular Matter 18.(4) (2016), p. 81 (cit. on p. 3).

[274] Jorge G Zornberg, Alexandre R Cabral, and Chardphoom ViratJANDR. "Behaviour of tire shred sand mixtures". In: Canadian Geotechnical Journal 41.(2) (2004), pp. 227-241 (cit. on pp. 3, 76). 



\section{Summary}

The subject of this thesis is the static elastic, solid-like behavior and its limits of dense granular materials. Granular materials, e.g. soil, are defined as a large number of grains or particles acting collectively as an ensemble. Particulate or granular media play crucial roles in both industry and nature. Understanding the elastic behavior of these media is crucial to our ability to improve the safety and efficiency of multitudinous industrial processes spanning almost all sectors, and is pivotal to the prediction and prevention of catastrophic natural phenomena such as earthquakes and landslides. However, unlike for classical media, there do not exist at present theoretical models capable of accurately predicting the behaviors of granular systems. Numerical models - though capable of quantitative accuracy - remain heavily reliant on precise calibration and experimental validation. As such, our ability to explore and hence better understand these systems depends heavily on experimental methods.

Understanding the effective mechanical properties of closely packed, dense granular systems is of interest in many fields, such as soil mechanics, materials science and physics. The main difficulty arises due to discreteness and disorder in granular materials at the particle scale which requires a multi-scale approach. The mechanical behavior of granular materials is highly nonlinear and involves plastic deformations also for very small strain due to rearrangements of particles. On the other hand, the concept of an initial purely elastic regime at small strains for granular assemblies is an issue still under debate in the community of soil mechanics. In addition, approaches that neglect the effect of elastic stored energy are also questionable, i.e., all the work done by the internal forces is dissipated.

Studying the mechanisms occurring at the grain level can improve our understanding of some complex aspects of soil behaviour. The aim of this thesis is to create a clear pathway toward the importance of micro- on macro-mechanical 
response at elastic level. The acquisition of more comprehensive, more detailed, higher-resolution data from particulate systems, should help to improve our understanding thereof. Remarkably, this dissertation attempts to frame and compare contributions from different points of view, numerics, experiments and theories.

For many geotechnical structures under working loads, the deformations are small. The regime of deformation where the behaviour can be considered linear elastic is infinitesimal, with nonlinear and irreversible effects present already at small strains. Nevertheless, characterisation of the stiffness of soils is important, as it provides an anchor on which to attach the subsequent stressstrain response. Features visible in experiments, like the propagation of acoustic waves, can hardly be described without considering an elastic regime. Mechanical waves are disturbances that propagate through space and time in a medium in which deformation leads to elastic restoring forces. This produces a transfer of momentum or energy from one point to another, usually involving little or no associated mass transport if the amplitude is small enough. Probing a material with (ultra) sound waves can give useful information on the state, the structure and the mechanical properties.

Numerical simulations, based on the Discrete Element Method, have revealed the utmost role of the microstructure plays for the elastic behaviour of granular soils. Following this evidence, microstructure-based continuum models have been developed. However, continuum mechanics are yet insufficient. Thus, it is essential to understand the effect of the grain level parameters, such as friction and cohesion between particles, size of constituent grains, and statistics of configurations using DEM simulations, in order to improve our understanding and establish some linkage between such dissipative micro- with continuum parameters.

Although, DEM offers deep micro-mechanical insights, macro-continuum models are more desirable for applications, where the macroscopic state variables can be obtained via micro-macro averaging procedures on small, representative elementary volumes. Examples, for continuum theories, with reduced complexity, are stochastic-based theoretical models for granular materials that predicts the propagation of vibrations; or a macroscopic continuum model based on micro-scale fluctuation information obtained by the DEM simulations. They can be applied to test, improve and optimize novel designs for materials, constructions, or landfill/soils. Such proposed/modified/improved constitutive laws can in future be implemented in a Finite Element platform to simulate selected larger i.e. continuum-scale problems.

To level up the complexity, one can consider mixtures of different species, 
in particular particles with different stiffness properties like stiff and soft. The analysis of the interplay of different species, soft and stiff, on the elastic moduli and the sound absorption behavior of granular aggregates and mixtures is still an open field of research. One of the important outcomes of experimental study of wave into granular mixtures is a fact that simple mixture rules can not be applied for the prediction of the effective acoustic properties of such particulate media; whereas standard DEM simulations do not predict well the mechanical response of such systems. To be able to reproduce a better agreement between simulations and experiments, an improved contact law that considers the large deformations of soft particles is needed. Multi-contact models are new approaches to tackle the the interaction between soft and stiff particles, which can improve not only qualitative but also quantitative results achieved by particle simulations. However, deeper understanding of the effective moduli that control the velocity dispersion and attenuation in soft-stiff mixtures is still lacking.

In a nutshell, the purpose of this dissertation is to improve the understanding of basic physical of mechanical mechanisms in particle systems and to guide further developments for new macroscopic constitutive models, starting from the micromechanical view point. 



\section{Acknowledgments}

This thesis represents not only my work; it is a milestone in my life. It contains the results of many experiences during this journey that has been nothing short but amazing. During the past four and half years in the Multi Scale Mechanics (MSM) group, at the University of Twente, right from the time I came for the interview, till this present time, I have enjoyed tremendous support, help and guidance from colleagues, family and friends. At this point, I would like to express my heartfelt thanks to everyone who helped in one way or the other during the course of completing this thesis. Without them, this journey would never have started and ended.

First of all, I am deeply grateful to my supervisor, Prof. Stefan Luding for coaching and hosting me in his group as a $\mathrm{PhD}$ student, as this greatly changes my career life. He motivated and encouraged me during the research and writing of this thesis. His patience, guidance and kind help and discussions in all aspects have made the past years an ever-good memory in my life. I have learned a lot from his extensive knowledge and experience in physics, mechanics, software use, and many brilliant and creative ideas both in science and daily life! His enthusiasm, patience, kindness and constant availability made it possible. Thank you for trusting in me and giving so much freedom, for being there when I needed you, and for the incredible collaboration opportunities. I also would like to express my sincere thanks to his wife, Gerlinde for her direct and indirect support as she had to miss you quite some evenings and weekends during these years.

I thank my co-advisor, Dr. Vanessa Magnanimo, your day-to-day advice, supervision and coaching has been really invaluable. I say out of profound gratitude that nothing of this thesis would have been possible without the incredible support from Vanessa. You have made me a better researcher and taught me the essentials of working within and outside the academic world. I appreciate your 
tremendous support, fruitful discussions and encouragement; I had a marvelous time working with you and look forward to collaborate with you in future.

The experimental part of the work was performed in the chair of Continuum Mechanics at University of Stuttgart; therefore, I would like to thank Prof. Holger Steeb for the nice collaboration and the great support. Your insight from an engineering point has broadened my vision and approach in dealing with the problem. Your were always been enthusiastic about new results and for the discussions thereafter. It is worth mentioning that my DFG proposal writing would have been incomplete without his massive support.

I would also like to thank my committee members for their interest in my research. In particular, Prof. Jim Jenkins for his fruitful scientific discussions. I acknowledge Prof. Gael Combe for your kind appreciation of my work; it was a pleasure to collaborate with you during the preparation of Alert book. And I would like to thank Prof. Ton van den Boogard and Prof. Andre de Boer for evaluating my thesis and being part of my graduation. The financial support from T-MAPPP, Marie Curie ITN, is gratefully acknowledged. To the T-MAPPP consortium - professors, advisors, colleagues and industrial partners - I say thank you for the time we shared together during the training in several countries. A special thanks to Dr. Antonia Larese for hosting me during my short secondments to the CIMNE, Barcelona.

I would like to extend my gratitude towards my former and present colleagues in the MSM group. Hao \& Giuseppe, you were the first colleagues I met in the group and we shared nice memories in the first year of my stay in Enschede. Sudesna, I say thanks for the time we had together in and outside research. Bert, I have special gratitude for taking out your precious time for helping with the samenvatting of this thesis. Kit, it was a great pleasure to share office with you. I was lucky to find someone who is as desperate as me for going to gym. Special thanks should go to Sylvia who arranges memorable events and helping me with the administration and other paper-works with kind and patience! Thank you Wouter den Breeijen for helping with any computer related problems and being available when needed. Furthermore, I would like to take this opportunity to thank Giacomo, Bram, Robert and Henk-Jan who trusted me to be their daily supervisor during their studies at University of Twente.

In no particular order, I would like to take this opportunity to thank my friends Rohit, Behzad, Somik, Alexander, Ahmad, Nicoleta, Ilaria, Kostas, and Tomaz, who walked shoulder to shoulder through this journey. You are indeed a friend in need, a friend indeed. Rohit, it is hard to find words to express my gratitude for your selfless friendship The uncountable overnight talks at my balcony with you are something I will always remember. All the scientific 
discussions I had with you were invigorating and helped me to sharpen my viewpoint. Behzad, accept my endless gratitude. I will never forget the days of my crisis when you have always been here right beside me. I have always looked forward to your visits to Enschede over the weekends, and we could never finish all the plans. Alexander, I had never imagined that you would be one of the closest friend to talk with about different subjects and for the loud laughs together. I adore your simplicity, honesty, and humor. I humbly thank you for all visits, those mean a lot to me. Tomaz, event though you did not stay long in T-MAPPP community, but that short time was enough to establish a constant friendship with you. I cherish spending time with you and discuss on diverse subjects. Kostas, the phenomenal collaboration with you has been of significant joy to me. I truly appreciate your care during the time we had in Greece. Ahmad, thank you for being always cool in difficult situations, and to believe in that its not the end, but just a new beginning. It was a pleasure to share office and time with you, and being not only my colleague but also a decent friend in times of my gloomy mood. Somik, it was so awesome of you to visit me time to time at my balcony. I have always admired your thoroughness and eye for detail and I have learned a lot from you; especially, to be gracious and gentle to everyone. Ilaria, who was always eager to listen to my stories, answering my surplus curious questions, and understanding me. I was honored to prepare work-package reports with you; furthermore, our collaboration was such a pleasant enjoyment in Barcelona and Enschede. Nikoleta, your generosity overwhelms me. You have been I thank you from the bottom of my heart. Your marriage is an unforgettable event in my memory.

I am also indebted to my friends Mitesh, Madhan, Vivek and Raghu for their friendship and support throughout this journey. Our friendship from masters time in Germany made me feel comfortable in the Netherlands. I extend my sincere thanks to my friends outside Twente, Abbas, Yusef, and Saeed, for helping me to cruise through the graduate life. You are the one on whom I could easily count on without a thought. Nevertheless, my thanks to my friends back in Iran, Alireza, Hamidreza, Pedram, Rasul, Ahmad, Hamed, Hadi, and Masud, who motivated me and encouraged to strive for higher goals. I am glad to have my gold old friends for being the closest distant friends. Finally, I am much obliged to Aysel for being there during the final hectic days of my PhD. You are the constant source of my motivation for the next move in my life.

Last but not least, I owe many thanks to my family. I am grateful to my parents for giving me their deep love, sacrifice, encouragement and support during my years of education. I want to thank my sister Shirin for believing on me more than myself and the care and warmth that you have provided. 



\title{
Curriculum Vitae
}

\section{Education}

\author{
27 Auguest 1990 : Date of Birth \\ 2008 - 2012 : B. E. in Mechanical Engineering \\ Malek-Ashtar University of Technology, Isfahan, Iran \\ 2012 - 2014 : M. Sc. in Computational Materials Science \\ TU Bergakademie, Freiberg, Germany \\ 2012 - 2019 : Ph. D. at the Multi-Scale Mechanics group \\ University of Twente, Netherlands
}

\section{List of Publications}

\section{Journals}

- Taghizadeh, K., Luding, S., \& Magnanimo, V. International Journal of Solids and Structures, submitted Aug. 2018.

- Taghizadeh, K., Steeb, H., Luding, S., \& Magnanimo, V. Journal of Mechanics and Physics of Solids, submitted Aug. 2019.

- Giannis, K., Taghizadeh, K., Schilde, C., Finke, J.H., Celigueta, M.A., Luding, S., \& Kwade, A. Granular Matter, submitted Jul. 2019.

- Shrivastava, R.K., Taghizadeh, K., \& Luding, S. Journal of Statistical Mechanics, submitted Sept. 2019. 
- Taghizadeh, K., Luding, S., \& Magnanimo, V. Physical Review E, to be submitted.

- Taghizadeh, K., Iaconeta, I., Larese, A., Magnanimo, V., \& Luding, S. Journal of Applied Mechanics, in preparation.

\section{Proceedings}

- Taghizadeh, K., Kumar, N., Magnanimo, V., \& Luding, S. (2015). Understanding the effects of inter-particle contact friction on the elastic moduli of granular materials. In IOP Conference Series: Earth and Environmental Science (Vol. 26, No. 1, p. 012008). IOP Publishing.

- Kievitsbosch, R., Smit, H., Magnanimo, V., Luding, S., \& Taghizadeh, K. (2017). Influence of dry cohesion on the micro-and macro-mechanical properties of dense polydisperse powders \& grains. In EPJ Web of Conferences (Vol. 140, p. 08016). EDP Sciences.

- Taghizadeh, K., Steeb, H., Magnanimo, V., \& Luding, S. (2017). Elastic waves in particulate glass-rubber mixture: experimental and numerical investigations/studies. In EPJ Web of Conferences (Vol. 140, p. 12019). EDP Sciences.

- Smit, H., Kievitsbosch, R., Magnanimo, V., Luding, S., \& Taghizadeh, K. (2018). Influence of Irreversible Contacts on the Stiffness of Dense Polydisperse Packings. In Micro to MACRO Mathematical Modelling in Soil Mechanics (pp. 327-336). Birkhäuser, Cham.

\section{Book Chapter}

Taghizadeh, K., Luding, S., \& Magnanimo, V. (2017). DEM applied to soil mechanics. ALERT Doctoral School 2017 Discrete Element Modeling, 129. 
